\title{
Development of 'Ready to Use Kits' for the simultaneous qualification and quantification of drugs in different matrices using Mass Spectrometry, expanded with the application of multimodal imaging techniques
}

Citation for published version (APA):

Genangeli, M. (2019). Development of 'Ready to Use Kits' for the simultaneous qualification and quantification of drugs in different matrices using Mass Spectrometry, expanded with the application of multimodal imaging techniques. [Doctoral Thesis, Maastricht University, University of Camerino]. Maastricht University. https://doi.org/10.26481/dis.20191203mg

Document status and date:

Published: 01/01/2019

DOI:

$10.26481 /$ dis.20191203mg

Document Version:

Publisher's PDF, also known as Version of record

\section{Please check the document version of this publication:}

- A submitted manuscript is the version of the article upon submission and before peer-review. There can be important differences between the submitted version and the official published version of record. People interested in the research are advised to contact the author for the final version of the publication, or visit the DOI to the publisher's website.

- The final author version and the galley proof are versions of the publication after peer review.

- The final published version features the final layout of the paper including the volume, issue and page numbers.

Link to publication

\footnotetext{
General rights rights.

- You may freely distribute the URL identifying the publication in the public portal. please follow below link for the End User Agreement:

www.umlib.nl/taverne-license

Take down policy

If you believe that this document breaches copyright please contact us at:

repository@maastrichtuniversity.nl

providing details and we will investigate your claim.
}

Copyright and moral rights for the publications made accessible in the public portal are retained by the authors and/or other copyright owners and it is a condition of accessing publications that users recognise and abide by the legal requirements associated with these

- Users may download and print one copy of any publication from the public portal for the purpose of private study or research.

- You may not further distribute the material or use it for any profit-making activity or commercial gain

If the publication is distributed under the terms of Article 25fa of the Dutch Copyright Act, indicated by the "Taverne" license above,

Download date: 26 Apr. 2023 


\begin{abstract}
Development of "Ready to Use Kits" for the simultaneous detection and quantification of drugs in different matrices using Mass Spectrometry, expanded with the application of multimodal imaging techniques.
\end{abstract}

Michele Genangeli 
ISBN/EAN: 978-94-6380-630-5

Cover design \& layout : Michele Genangeli

Printed by: Proefschriftmaken (www.proefschriftmaken.nl)

(c) Michele Genangeli, Maastricht, The Netherlands, 2019

Paper copies are available trough the library of the University of Camerino and Maastricht University. 
Development of "Ready to Use Kits" for the simultaneous

qualification and quantification of drugs in different matrices using Mass Spectrometry, expanded with the application of multimodal imaging techniques.

\section{DISSERTATION}

To obtain the doctorate degree from Maastricht University, on the authority of the Rector Magnificus

Prof dr. Rianne M. Letschert,

In accordance with the decision of the Board of Deans,

To be defended in public

On Tuesday $3^{\text {rd }}$ of December 2019 at 14:00 hours

By

Michele Genangeli

Born in Jesi (AN), Italy, on September 29th 1990 


\section{Promoters:}

Prof. Dr. R.M.A. Heeren

Prof.Dr. Sauro Vittori

\section{Co-promoter (Copromotor)}

Dr. Tiffany Porta Siegel

\section{Assessment committee (Beoordelingscommissie)}

Prof. Dr. Maarten Honing, Professor of Analytics in Systems Biology, Maastricht University (chairman);

Prof. Dr. Martijn Poeze, Professor of Surgery, especially Trauma Surgery, Maastricht University;

Prof. Dr. Michel Nielen, Professor of Analytical Chemistry, Wageningen University \& Research;

Prof. Dr. Garry Corthals, Professor of Biomolecular Systems Analytics, University of Amsterdam;

Prof. Dr. Enrico Marcantoni, Professor of Organic Chemistry, University of Camerino, Italy

Prof. Dr. Piera di Martino, Professor of Pharmaceutical Technological Application, University of Camerino, Italy. 
This research was part of the M4I research program financially supported by the Dutch Province of Limburg through the LINK program.

This research was also part of the "Eureka" research program financially supported by the Italian Marche region, Italy.

The author acknowledges receipt of a European Commission funded H2020 MSCA-ETN grant under proposal number 675743 (project acronym: ISPIC).

The author also thank Eureka Lab Division (Company) and University of Camerino for financial support when in Italy. 

To my lovely grandfather \& to all the people who love me. 


\section{Table of contents}

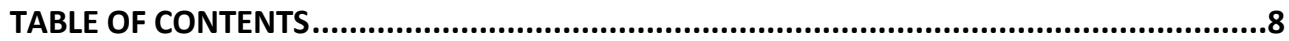

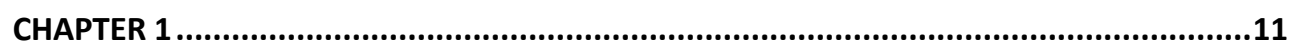

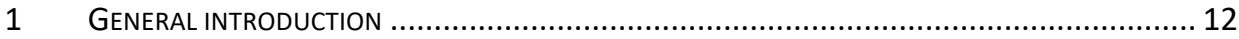

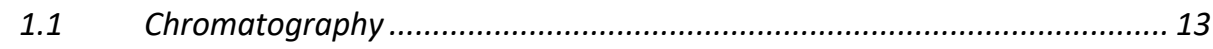

1.2 Mass spectrometry (MS) and mass spectrometry imaging (MSI) ............... 14

1.3 Preparation of biological samples for LC-MS applications ......................... 27

1.4 Preparation of biological samples for MSI applications ............................. 28

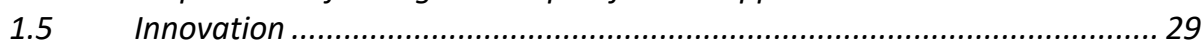

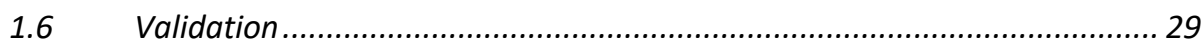

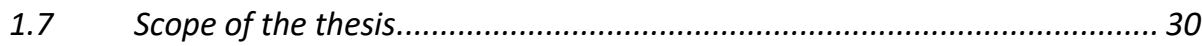

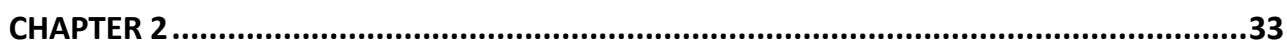

2 LIQUID CHROMATOGRAPHY-TANDEM MASS SPECTROMETRY IN LIQUID CLINICAL SAMPLES FOR

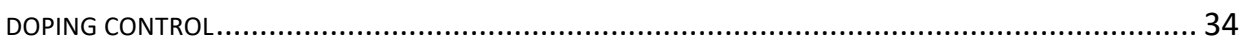

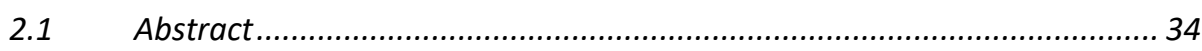

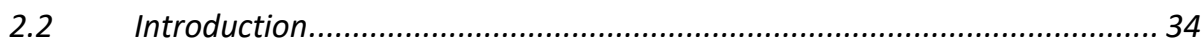

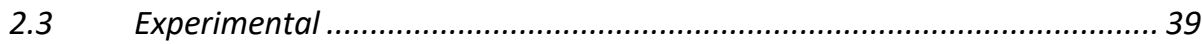

2.4 Development and application of a UHPLC-MS/MS method for the simultaneous determination of 17 steroidal hormones in equine serum ................... 49 2.5 Qualification and quantification of 17 endogenous and exogenous steroidal hormones in equine and bovine blood for doping control with UHPLC-MS/MS: a new method. 60

2.6 Simultaneous quantitation of 9 anabolic and natural steroidal hormones in

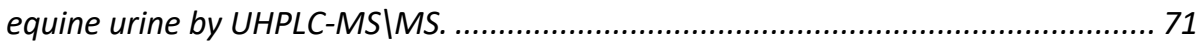

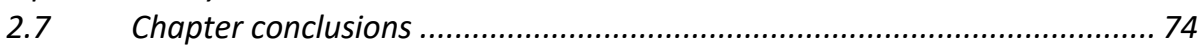

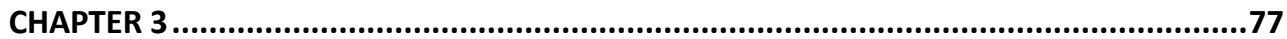

3 MASS SPECTROMETRY IMAGING FOR A BETTER UNDERSTANDING OF BIOLOGICAL PATHWAYS..... 78

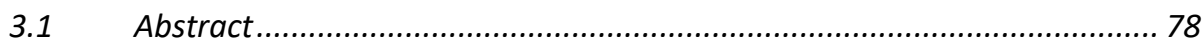

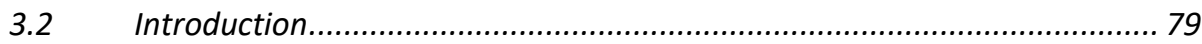

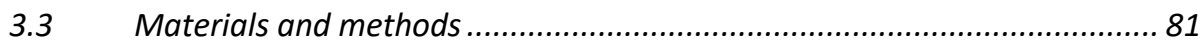

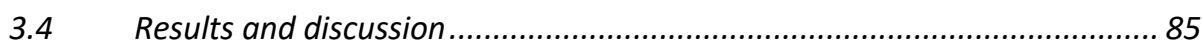

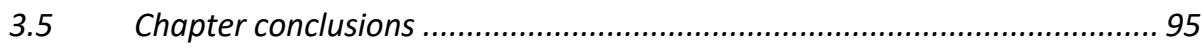

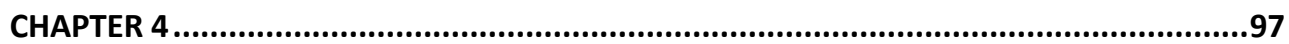

4 FFPE TISSUE-BASED QUALITY CONTROL FOR MALDI-MSI ANALYSIS OF TRYPTIC PEPTIDES........ 98

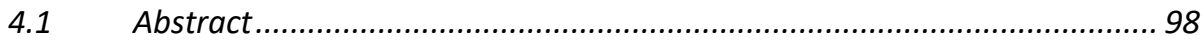




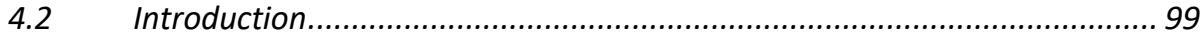

4.3 Experimental section ......................................................................... 101

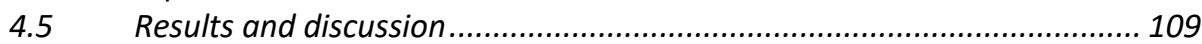

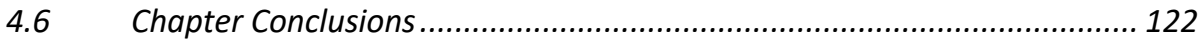

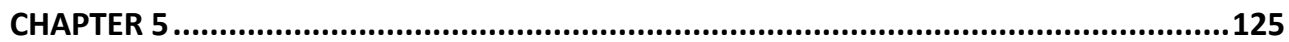

5 TISSUE CLASSIFICATION BY RAPID EVAPORATIVE IONIZATION MASS SPECTROMETRY (REIMS): COMPARISON BETWEEN A DIATHERMIC KNIFE AND $\mathrm{CO}_{2}$ LASER SAMPLING ON CLASSIFICATION

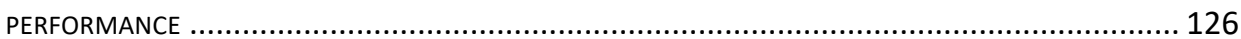

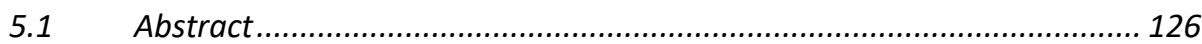

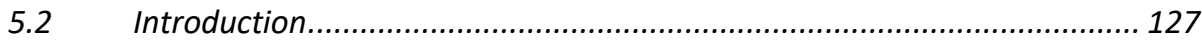

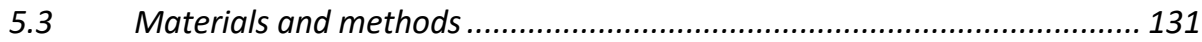

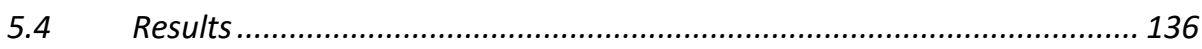

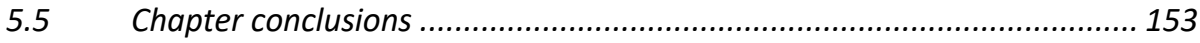

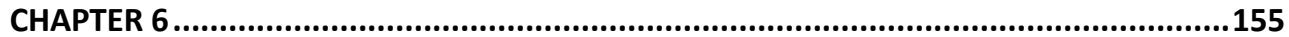

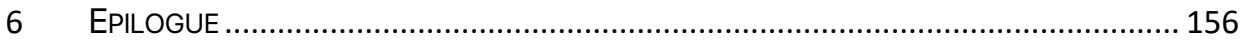

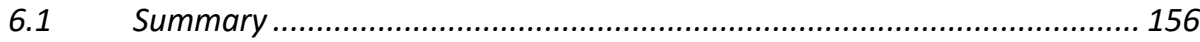

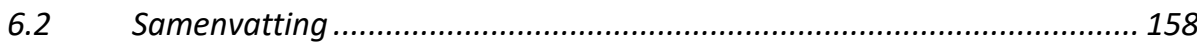

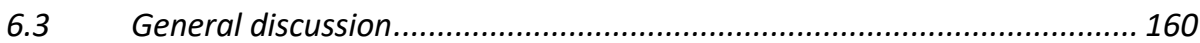

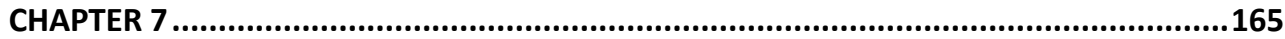

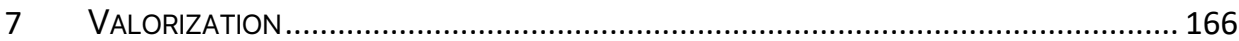

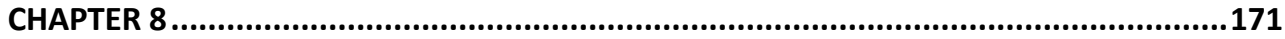

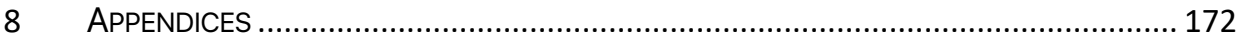

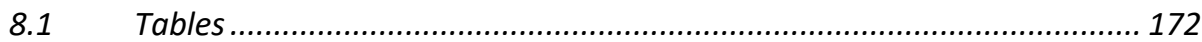

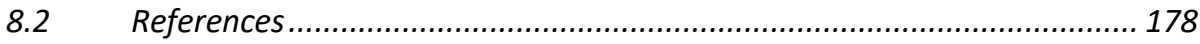

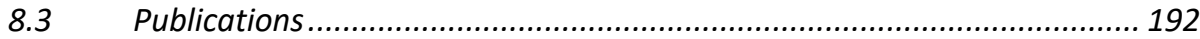

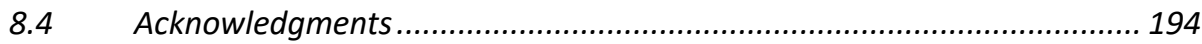

About the Author................................................................................ 197 



\section{Chapter 1}

General introduction
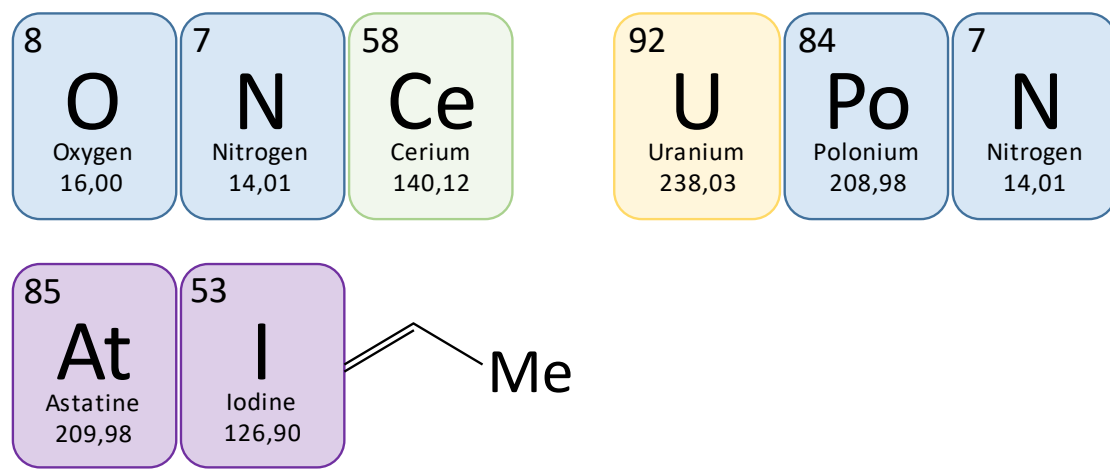


\section{General introduction}

Mass spectrometry (MS) is a versatile technique with an interdisciplinary nature and broad applications that combines physics, chemistry and biology. A mass spectrometer is considered as the smallest weighing scale in the world [1], suited to localize, identify and quantify big and small molecules as well as single atoms and isotopes. These features make MS an incredible scientific tool for both biological and non-biological sample analysis.

Since its invention during the first decade of $20^{\text {th }}$ century, MS has undergone tremendous improvements regarding sensitivity, resolution, mass range and sampling techniques. MS can now be used to precisely quantify molecules and study the spatial distribution of proteins, peptides, lipids, metabolites or drugs. These improvements led to an increase in applications of this technique in all scientific disciplines such as chemistry, physics, biology, pharmacology, medicine, biochemistry and food industries.

This thesis explores the different applications of mass spectrometry such as qualification and quantification of different molecules in human and animal biological samples (solid and liquid) and a multimodal approach for mass spectrometry imaging (MSI) of solid/intact tissues. Developments achieved within this thesis include sample preparation, qualification, quantification of detected molecules with high sensitivity and high spatial resolution. High-throughput detection techniques and fast sample preparation have also been investigated to facilitate the implementation of the developed methods to the clinic. 


\subsection{Chromatography}

The Russian botanist Mikhail Tswett first developed chromatography (from

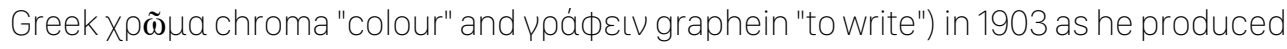
a colorful separation of plant pigments through a column of calcium carbonate. Chromatography has since developed into an invaluable laboratory tool for the separation and identification of compounds [2]. Although color usually no longer plays a role in the process, the same principles of chromatography still apply.

A mixture/solution (analyte) is dissolved in a fluid called the mobile phase, which carries it through a structure holding another material called the stationary phase. For this thesis (Chapter 2), it was used a hydrophobic stationary phase made of octadecyl carbon chains (C18). This type of chromatography is called reversed-phase chromatography (RPC) where the separation occurs due to the differential partitioning of the analytes between the mobile and stationary phases. Instead of using salt gradients to elute hydrophobic species, organic modifiers (e.g. acetonitrile or methanol) are gradually added to the elution buffer. This weakens the hydrophobic attraction between a stationary phase and hydrophobic species separating the mixture. Chromatography can be performed with both liquid and gas samples. 


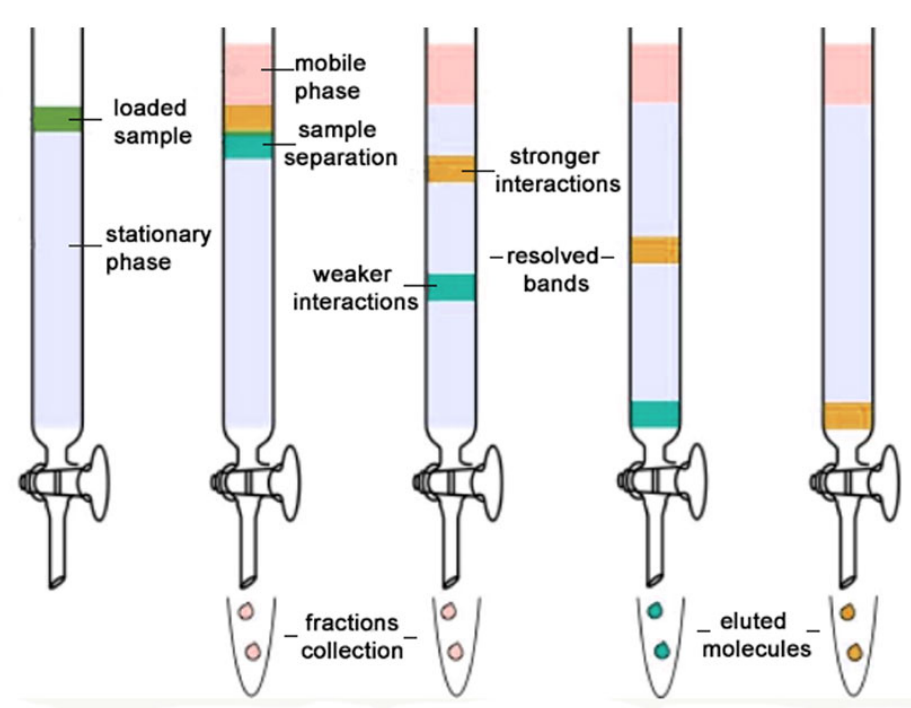

Figure 1 - Illustration of the chromatographic separation of analytes. The sample is loaded in the column. The mobile phase gradually and differently elutes the analytes through the end of the column, separating the analytes. (Figure taken from https://prepgenie.com.au/gamsat/column-chromatography/)

HPLC is an acronym that stands for "high-pressure liquid chromatography" or "high performances liquid chromatography" and represents the evolution of a classic liquid chromatography system. HPLC follows the same separation principles but with higher pressure (more than 50 Bar) leading to better efficiency and faster separation than traditional liquid chromatography (LC)[3]. In this thesis, ultra high-pressure liquid chromatography (UHPLC) system was used. The UHPLC can withstand higher pressures than a standard HPLC (>50 bar) allowing the usage of sub 2-micron columns, leading to overall better performances and shorter analytical time.

\subsection{Mass spectrometry (MS) and mass spectrometry imaging (MSI)}

Mass spectrometry (MS) was originally developed in the early $20^{\text {th }}$ century to measure the masses of atoms leading to the discovery and confirmation of the existence of isotopes [4]. Since then, the number of mass spectrometrists and mass spectrometers rapidly expanded. Until the 1940s, the MS field was dominated by physicists trying to resolve questions regarding the 
fundamental nature of the atom [4]. Only a few years after, MS started being applied to the quantitation of substances, leading to an exponential increase of its applications [4].

A mass spectrometer can be identified by three major components: an ion source (that produced gaseous ions from the substance being studied), a mass analyzer (which resolves the ion into their characteristics mass components according to their mass-to-charge ratio) and a detector (for the detection of ions and recording the relative abundance of each of the resolved ionic species) [5]. The introduction of "soft" ionization techniques, such as electrospray ionization (ESI) by Fenn et al. [6] and matrix-assisted laser desorption ionization (MALDI) by Karas et al. [7], revolutionized mass spectrometry as it offered the possibility to analyze large intact biomolecules. As such MS became an irreplaceable tool for the biological sciences. The molecular weight (MW) ranges we use in this thesis are defined as follows. The low Mw range includes elements and molecules from 1 to 500 Da. Molecules with Mw between 500 and 2000 Da fall in the medium Mw range. All molecules with Mw > 2000 Da are considered to be part of the high molecular weight class.

Chapter 3 and 4 , focuses on one of the lastest, rapidly developing innovations in MS: mass spectrometry imaging (MSI).

MSI allows for rapid detection, localization and identification of a tremendous amount of molecules from the most complex biological samples. Advantages of MSI over traditional MS: it is a label-freetechnique which can provide a detailed understanding of biological processes from sub-cellular to multicellular level.

The several ionization techniques, mass analyzers and detectors used in this thesis are listed and basically explained in the following section. A detailed explanation of every ionization technique, mass analyzer and ion detector is beyond the scope of this thesis. 


\subsubsection{Basic principles of lonization techniques, mass analyzers and ion detectors}

A mass spectrometer uses electric and magnetic fields to "control" and deflect ionized (positively or negatively charged) atoms and molecules.

The molecules are ionized by the ion source. There are many types of ionization techniques currently used in mass spectrometry with several ionization sources able to ionize molecules from almost every material. Once ionized, the ions are then transported or separated by magnetic or electric fields (mass analyzers) and lastly detected.

The different ionization techniques/sources, as well as mass analyzers and detectors used for the work presented in this thesis, are listed in the subsections below

\section{Electrospray lonization (ESI)}

Electrospray ionization is considered a "soft" ionization technique and it is the most common ionization technique coupled with LC. It was firstly reported by Yamashita and Fenn in 1984 and a Nobel prize was attributed to Fenn in 2002 [6, 8]. This "soft" ionization produces very little ion excitation and thus little or no fragmentation of the analyte occurs [9]. Ions are generated by introducing a liquid sample into a chamber via a hypodermic needle where an electrical potential difference (generally $2-4 \mathrm{kV}$ ) between the needle inlet and a metal surface in front of it promotes ionization of the emerging liquid and disperses it into charged droplets [10]. 


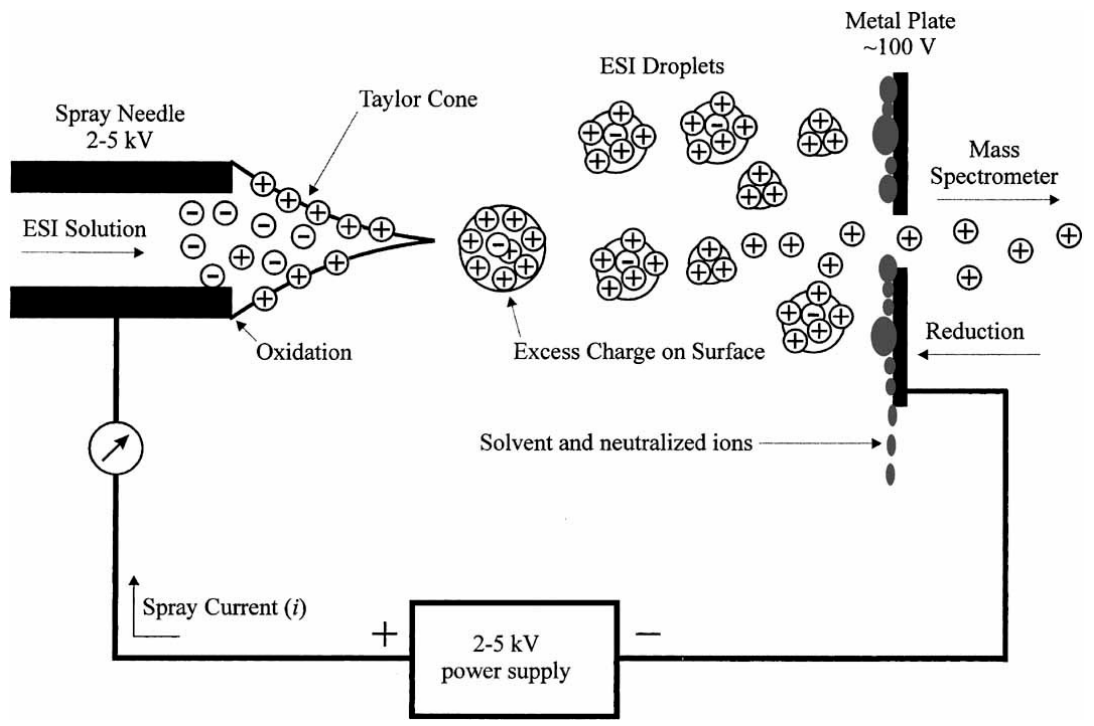

Figure 2 - Schematic of the electrospray ionization process. The liquid is sprayed creating charged droplets. The solvent evaporates and ions are ejected from the highly charged droplets. (Figure taken from Cech et al. [11])

Two major theories explain the final formation of ions: the ion evaporation model (IEM) and the charge residue model (CRM), and the explanation of these models goes beyond the scope of this thesis. Briefly, solvent desolvation and evaporation upon heat transfer from the ambient gas leads to shrinking of the droplets and the accumulation of excess surface charge. After reaching a certain limit (Rayleigh limit), the electric field becomes high enough (up to $109 \mathrm{~V} / \mathrm{cm}$ ) to desorb analyte ions through a process called coulomb fission [12]. ESI can be operated in either positive (+) and negative (-) modalities and the ions observed are generally generated by addition or removal of hydrogen cation $[\mathrm{M}+\mathrm{H}]+$ and $[\mathrm{M}+\mathrm{H}]-$ or sodium, ammonium addition $[\mathrm{M}+\mathrm{Na}]+$ and $[\mathrm{M}+\mathrm{NH} 4]+$ as well as anion addition (e.g. $[\mathrm{M}+\mathrm{Cl}]-$ ). Multiply charged ions such as $[\mathrm{M}+\mathrm{nH}] \mathrm{n}+$ are often observed.

In this thesis, the ESI source was used in combination with a UHPLC system and a triple quadrupole mass spectrometer (see next paragraph) in Chapter 2 and partly in Chapter 3. Chapter 2 reports the beforementioned instrumental setup to precisely quantify and qualify steroids in serum, blood and urine, while Chapter 3 uses the same set up to quantify and qualify bile acids, cholesterol in rat feces and blood respectively. 


\section{Matrix-assisted laser desorption/ionization (MALDI)}

The most frequently employed ionization technique for MSI is MALDI, due to its suitability for direct detection of a wide variety of molecules such as proteins and peptides [13-17], lipids, drugs and metabolites from a wide range of complex biological and non-biological surfaces. MALDI is a dramatic improvement of laser desorption/ionization mass spectrometry (LDI-MS). MALDI is an ionization technique capable of producing intact higher Mw ions through the use of a pulsed laser beam combined with an energy-absorbing matrix.

To generate ions, MALDI requires the sample surface to be coated with a chemical matrix, typically a small organic compound which allows the extraction and "soft" ionization from the laser (desorption). The role of the matrix is to absorb the majority of the laser energy leading to explosive desorption of the matrix crystals together with incorporated analyte into the gas phase without degradation of the analyte. The matrix also aids the ionization of analyte molecules in the gas phase due to the presence of protons from added acids such as trifluoroacetic acid (TFA) [18]. Different matrices are currently used according to their affinity to the compounds of interest. The matrix must be applied homogeneously to avoid delocalization and nowadays several techniques allow the application of matrices routinely. A matrix solution can be sprayed, nebulized or sublimated on top of the tissue.

The spatial resolution or pixel size achievable by focusing the laser beam varies between 1 and 100 micrometers, depending on the system employed.The laser is generally a N2 (337 nm) or neodymium-doped yttrium aluminum garnet (Nd:YAG) (355 nm) with repetition rates of 200-1000 Hz and typical pulse lengths of 3 ns or less [19]. The laser is fired on the matrix instantly desorbs the molecules extracted from the tissue by the matrix; these molecules undergo a series of reactions that ultimately lead to the ionized analyte (singly protonated $\left.[\mathrm{M}+\mathrm{H}]^{+}\right)$. 


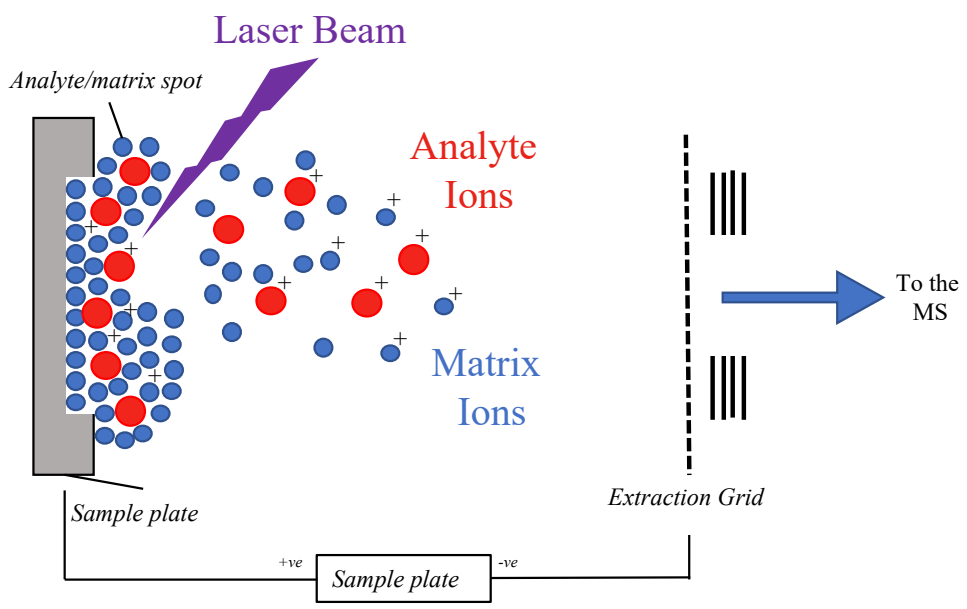

Figure 3 A schematic representation of a matrix-assisted laser desorption/ionization process (Figure realized by the author)

For imaging, the sample is typically moved after every laser shot while the position of the laser itself is kept constant. A mass spectrum Is recorded at every position and each of the mass spectrum represents a pixel in the 2D image. Modern MALDI systems utilize laser scanning mirrors simultaneously while moving the sample to increase the acquisition speed approaching 40 pixels/second [20].

MALDI-MSI is a leading method for recording the distribution of intact molecules. It can detect hundreds of compounds directly from tissue sections due to its high sensitivity, large tolerance for salts and other contaminants, a wide mass range, little fragmentation, label-free detection method and easy data interpretation because the majority of ions are singly protonated.

MALDI-MSI is used in Chapter 4 and, in combination with LC-MS, in Chapter 3.

\section{Rapid evaporative ionization mass spectrometry (REIMS)}

REIMS is a relatively new ambient ionization technique that has already brought new sampling methods into a wide range of applications [21]. involves the aspiration 
and rapid thermal ionization of aerosols produced from electrocautery, ultrasonic aspiration [22], ultraviolet (UV) and infrared (IR) lasers [23-26].

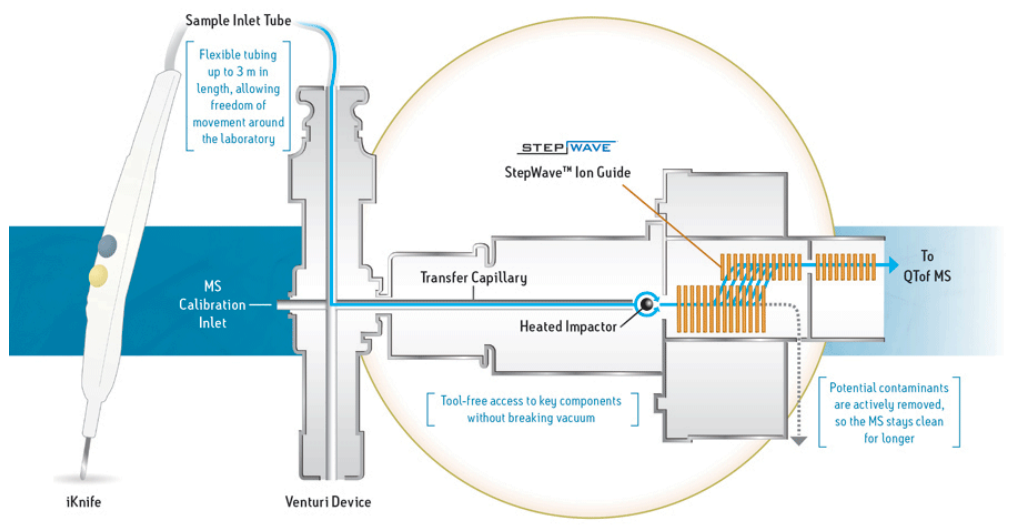

Figure 4 - Schematic description of the REMS system (Figure taken from http://mww.waters.com/REMS-ResearchSystem-with-iKnife-Sampling)

The most common handpieces employed to generate smoke and combined with a REIMS system are monopolar and bipolar electrocautery/diathermic (blade/needle) knives routinely used in the OR for surgical procedures [24]. A monopolar device uses a higher voltage when compared to a bipolar device and has a better ability to cut and coagulate large bleeding areas. Thus, a monopolar accessory is mainly used to cut and coagulate relatively large tissues while the bipolar electrocautery is more used for smaller tissue applications (e.g. brain surgery) or for the classification/identification of bacteria and fungi [27, 28] [29].

Amongst other certified medical devices, a $\mathrm{CO}_{2}$ laser [21] can also be used intraoperatively, mainly due to its infrared wavelength of $10.6 \mu \mathrm{m}$ highly absorbed in water molecules contained in tissue [30-32]

Coupling REIMS with a surgical laser handpiece that is already frequently employed in a surgical setting, does not require any modifications of the surgical procedures while offers the opportunity to the surgeon to make betterinformed decisions. 
REIMS was originally developed for oncological surgical applications [24, 33, 34] with the ultimate goal to improve patient outcome after surgery by ensuring the removal of all malignant tissue. The applicability of REIMS for cancerous tissue diagnostics has been demonstrated both ex vivoand in vivo in the operating room with instantaneous tissue classification ("normal"/surrounding tissue vs. tumor or tumor margins). The ex vivo procedure entails a surgical resection of biological material, followed by a REIMS enabled assesment of the tissue outside the patient, while the in vivo studies are conducted during resection of the material from the patient.

Chapter 5 reports the benefits and limitations of two surgical handpieces couple to REIMS: a $\mathrm{CO}_{2}$ laser and a diathermic knife to generate smoke from fresh animal tissues.

\section{Quadrupole and Triple quadrupole (QQQ) mass analyzers}

A triple-quadrupole mass analyzer (Q-qcoll-Q) consists of two quadrupole mass analyzers, connected by means of a collision cell, which is an RF-only quadrupole device [35]. These types of mass analyzers is often used when higher sensitivity and specificity are required, such as for the detection and quantification of traces of substances in complex matrices (Chapter 2). Furthermore, they may also be used to generate additional fragmentation data from ions of interest.

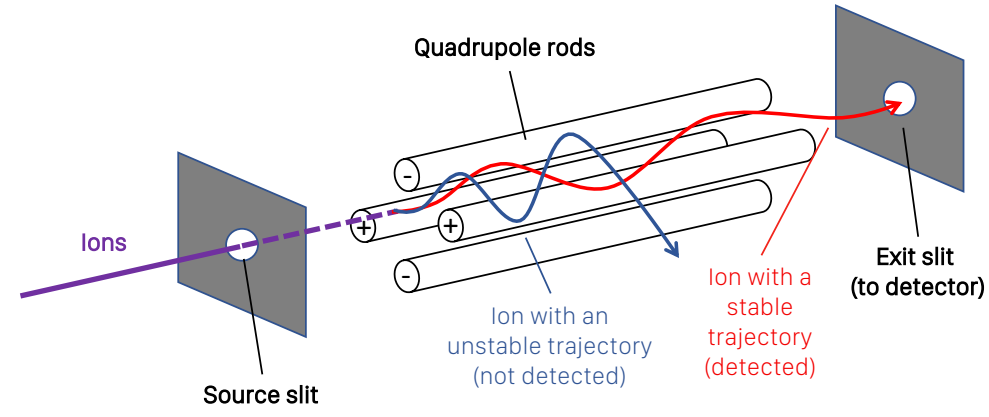

Figure 5 - Schematic representation of a Quadrupole mass analyzer (Figure realized by the author)

The instruments offer approximately $0.1 \mathrm{~m} / \mathrm{z}$ resolution and exhibit a linear response. The resulting MS/MS spectra can be helpful in structural identification and drug qualification and quantification. 
Triple-quadrupole instruments are quite flexible and allow the user to monitor and collect simple MS data, full-scan fragmentation data, multiple ion monitoring (MRM, Chapter 2), specific ion fragmentation data (selected reaction monitoring or SRM), neutral loss data and precursor ion data [36].

In MRM modality, the first quadrupole (Q1) selects ions of interest generated in the ion source and works as a filter for other ions besides the ones of interest. The second quadrupole (Q2) is typically filled with nitrogen or argon (denser than nitrogen leading to a more robust fragmentation) and is used as a collision chamber to generate fragment or daughter ions. The third quadrupole (Q3) is used to select and conduct specified fragment ions to the electron multiplier [36].

The generalized approach for a tandem MS analysis is therefore to select the target molecular ion's m/z value in Q1 and set Q3 to monitor the predetermined $\mathrm{m} / \mathrm{z}$ value(s) for the fragment ion(s). This approach is commonly referred to as multiple reaction monitoring (MRM).

\section{Time of Flight (TOF)}

Time-of-Flight (TOF) mass analyzers are the most common mass analyzers used for mass spectrometry imaging, particularly coupled with MALDI and DESI sources. The TOF provides high analysis speed, sensitivity and good mass resolution and mass accuracy [37]. This combination makes it particularly suitable for high-throughput MSI screening in a clinical setting by providing fast analysis and full spectra information. 


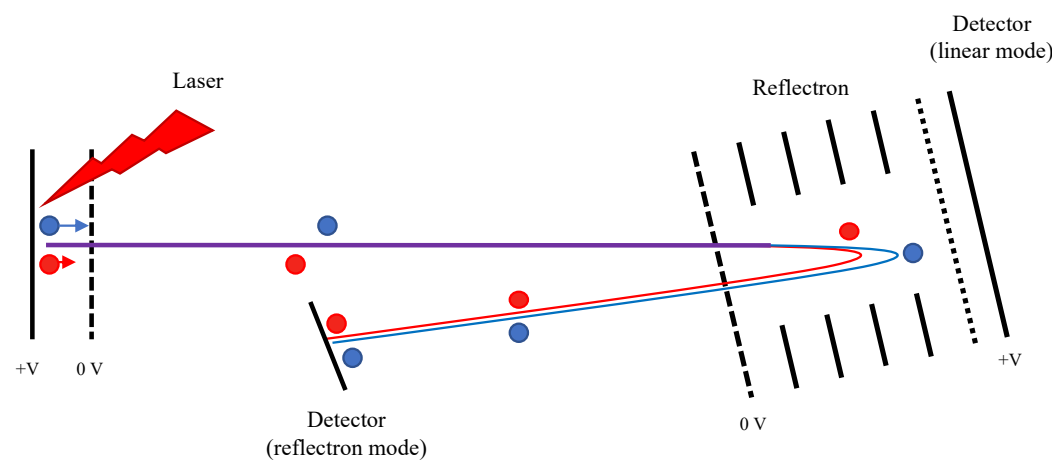

Figure 6 - Schematic representation of a TOF mass analyzer (Figure realized by the author)

The principle of TOF is straightforward: The generated ions are accelerated to the same kinetic energy and travel through a field-free region in a well-defined TOF tube under vacuum (typically 1-2 meters).

The main advantage of a TOF analyzer is that all the ions will eventually reach the detector (unlike quadrupole or sector instruments) [38].

The equation that correlates the mass to charge ratio $(\mathrm{m} / \mathrm{z})$ to the time of flight $\left(f_{t}\right)$ is:

$$
\mathrm{m} / \mathrm{z}=\mathrm{t}_{\mathrm{f}}^{2} 2 \mathrm{E} /(2 \mathrm{~s}=\mathrm{x})
$$

where $E$ is the voltage applied, $s$ is the length of the TOF tube, and $x$ is the length of the free-flight region.

Modern TOF-MS analyzers have both linear and reflectron TOF. The reflectron provides the advantage of increasing the length of the "free flight" region without the need of a longer tube and diminishes the spread of flight time of ions with the same $\mathrm{m} / \mathrm{z}$ (isobaric species). This results in a better mass resolution as all ions hit the detector at the end of the flight tube simultaneously and are time focused. The arrival time of all ions is recorded by the detector and is converted into an electronic signal by a combination of an amplifier and time-to-digital converter (TDC) or a fast analogto-digital converter (ADC). Detectors are usually either a secondary emission 
multiplier (SEM) or a multichannel plate (MCP) that produces an electron shower for every ion hit. The signal of the SEM or MCP is monitored as a function of arrival time with a resolution up to 138 ps. This setup is commonly used in a pixel-by-pixel approach of MSI, where all ions produced from one ablated spot on the sample surface are recorded on the detector as one spectrum.

In Chapters 3 and 4, a Rapiflex (MALDI-TOF instrument), operating in reflectron mode is employed to analyze the samples, while in Chapter 5, two handpieces are coupled to another TOF instrument which combines a REIMS source and a Q-TOF mass analyzer.

\section{Orbitrap}

The Orbitrap is an ion trap mass analyzer consisting of two outer electrodes and a central electrode, which enable it to act as both an analyzer and detector[39]. Ions enter the orbitrap and are trapped not with a radiofrequency or a magnet (as for the conventional ion traps), but with an electric field called "electrodynamic squeezing" [40]. The centrifugal force of the ions spinning around the central electrode of the Orbitrap compensates the electrostatic attraction, forcing them to move in complex spiral patterns. Ions with different $\mathrm{m} / \mathrm{z}$ ratio swing at different frequencies. By measuring the oscillation frequencies induced by ions on the outer electrodes, the mass spectra of the ions are acquired using image current detection. Due to its setup, the Orbitrap mass analyzer is actually a Fourier Transform mass analyzer analog of FT-ion cyclotron resonance (ICR) technology, yet with smaller instrument size and easier instrument operation [39].

A Fourier transform is employed to derive the frequencies of oscillations for the ions, providing an accurate reading of their $\mathrm{m} / \mathrm{z}$. 


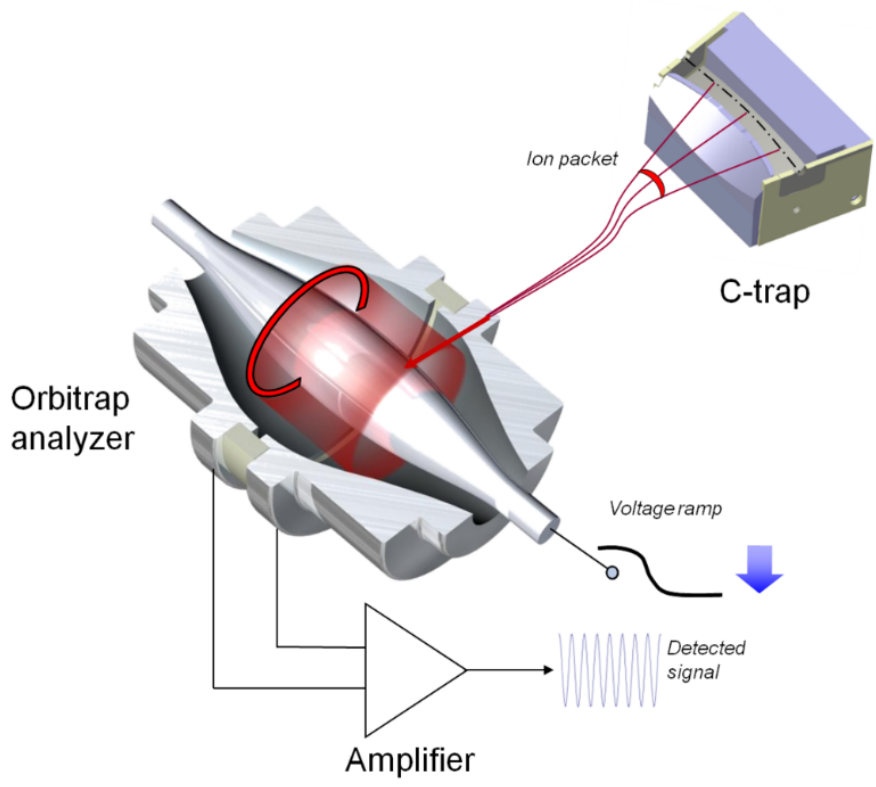

Figure 7 - Schematic representation of an Orbitrap system (Figure taken from Thermo Fisher Scientific)

Orbitrap mass spectrometers deliver an extremely high mass resolution in terms of full width at half maximum (FWHM) of 1,000,000 at m/z 200 and sub- 1 ppm mass accuracy in a single compact and easy-to-use instrument.

The combination of high resolution and mass accuracy is crucial to identify compounds of interest in complex matrices. In this thesis, the Orbitrap was used to identify lipidic species in chapter 5 with high sensitivity, accuracy and mass resolution.

\section{Fourier transform ion cyclotron resonance (FTICR)}

As described by Marshall et al. [41-43], FTICR - mass spectrometry (also called FTICR-MS), relies on the use of a strong, fixed magnetic field to deflect ions converting ionic mass into a cyclotron frequency. In order to confine the ions inside the FT chamber and avoid them from traveling along a helical path (being lost from the detecting region), a potential is applied to two plates perpendicular to the magnetic field [44]. Ions move with a cyclotron motion with a specific frequency according to 
their $\mathrm{m} / \mathrm{z}$ ratio and the intensity of the electric field applied. Variating the electric field while keeping the magnetic field constant allows to selectively detect ions with different $\mathrm{m} / \mathrm{z}$ ratio with extreme sensitivity.

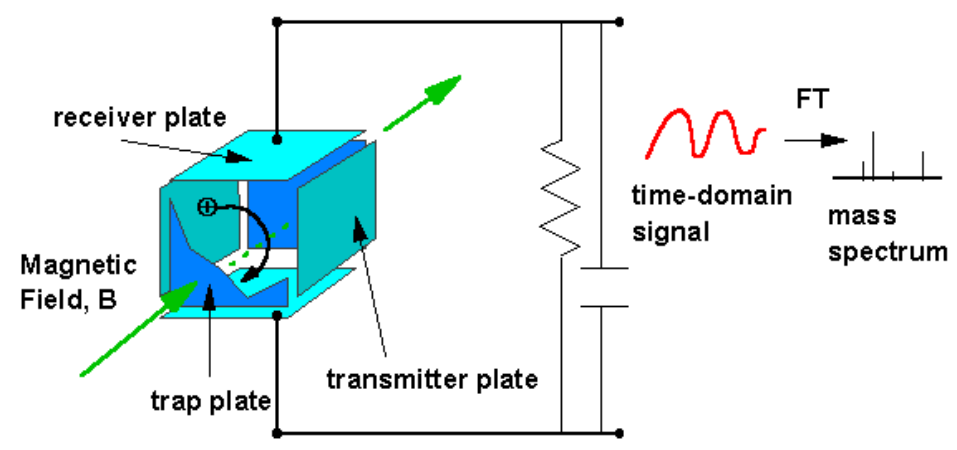

Figure 8 - A schematic representation of a Fourier transform ion cyclotron resonance mass analyzer - FTICR. (Figure Taken fromhttp://mww.mslab.ulg.ac.be/mslab/equipment/fticr-ms-bruker-apex-qe-9-4t/).

FTICR mass analyzers, allow the determination of ions with extreme resolution (>1,000,000 FWHM at $m / z 700)$ and mass accuracy ( $<2$ ppm). These performances make this technique an extremely powerful tool for small molecules analysis such as proteins or peptides, lipids and metabolites.

As well as for the Orbitrap, the FTICR was used to confirm lipidic species in chapter 5 with high sensitivity, accuracy and mass resolution. 


\subsection{Preparation of biological samples for LC-MS applications}

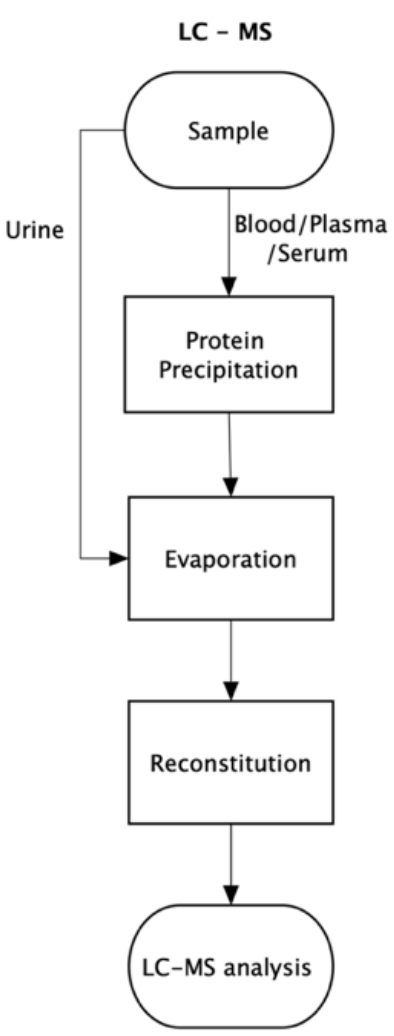

Figure 9-Schematic workflow of the sample preparation for LC-MS developed methodologies.

According to the type of biological sample and the technique/mass spectrometer used to quantify or identify the compounds of interest, we had to follow different sample preparation methods. In Chapter 2 we analyzed liquid biological samples (blood, serum and urine) with liquid chromatography-tandem mass spectrometry. The sample preparation for these complex liquid biological samples generally required deproteinization (I.e. via protein precipitation) and concentration of the samples. Specifically, blood and serum first underwent fast deproteinization using organic solvents. The lack of proteins in the urine did not require any prior deproteinization. After this step, all the samples had to be evaporated and restored with a small amount of solvent in order to concentrate the samples and increase the sensitivity of the method.

The methods described in Chapter 2 were developed considering the total time and complexity of the sample preparation. The goal was to develop fast, intuitive, sensitive and robust methods while keeping the sample preparation easy and as less time consuming as possible. A long and complex sample preparation increases the risk of mistakes from the operators and is not applicable for routinely applications such as doping control.

The detailed sample preparations will be discussed in every chapter of this thesis. 


\subsection{Preparation of biological samples for MSI applications}

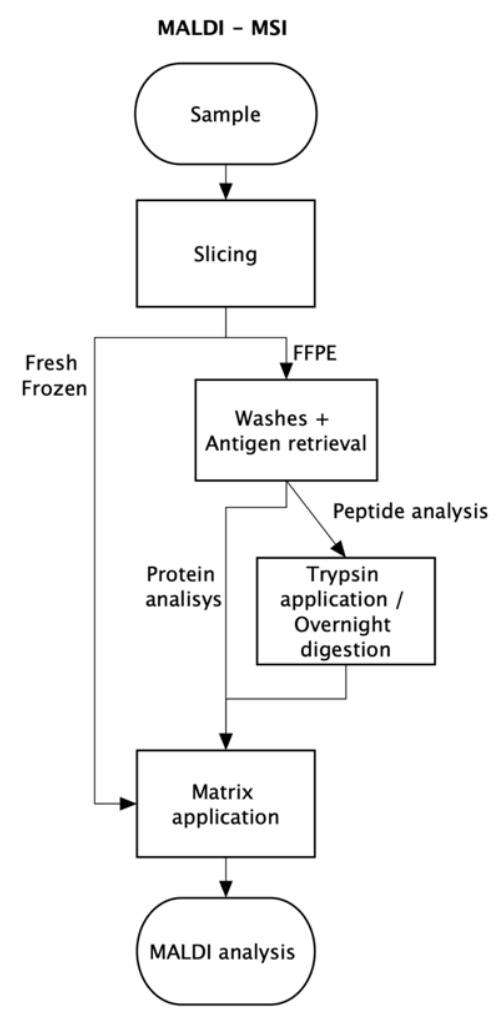

Figure 10 - Schematic workflow of the sample preparation for MALDI - MSI
The sample preparation for MSI analysis varies according to the type of sample being analyzed and the type of ionization source used.

Tissues are generally either frozen in liquid nitrogen after resection/ collection (fresh frozen tissues [FF]) or fixated with formalin and embedded in paraffin (FFPE). Both the sample types have advantages and disadvantages.

Fresh frozen tissue can be stored at $-80{ }^{\circ} \mathrm{C}$ for a limited amount of time, and ideal for any type of analysis. On the other hand, FFPE tissues can be stored for years but are not suitable for the analysis of lipids due to the paraffin.

Prior analysis, both types of samples must be cut in thin slices (5-20 $\mu \mathrm{m}$ ) with a cryotome (FF) or a microtome (FFPE) and mounted onto histological glass slides. Once sliced, FF tissue can be thawed and directly analyzed using DESI, or covered with a matrix for MALDI analysis. On the other hand, FFPE tissues must undergo a series of washes in order to remove the paraffin, digested (only for the analysis of peptides) and covered with a compound-specific matrix.

Lastly, REIMS does not require any sample preparation and allows the direct ambient analysis of fresh/intact tissues.

The detailed sample preparations specific for each technique will be discussed in every chapter of this thesis. 


\subsection{Innovation}

Mass spectrometry is continuously evolving and advancing as an analytical technique being applied to new fields every day. Researchers have to adapt and discover new ways to prepare and analyze an extremely wide variety of samples and trying to couple mass spectrometry with existing techniques, expanding its field of applications.

One example of MS "innovations" is reported in Chapter 5. REIMS, as other techniques in mass spectrometry, is trying to close the gap between the analytical world and "in vivo" applications such as surgery (human and veterinary).

Another example is reported in Chapter 4 where the development of a quality control tissue for FFPE analysis of breast cancer specimens and the usage of high throughput techniques shortens the gap between MSI and clinical applications. The challenge now is to keep designing and improving procedures that meet the demands for specific scientific questions and scientific needs while making these methods robust and reliable.

\subsection{Validation}

The methods reported in Chapter 2 have been developed and fully validated according to the guidelines from "Guidance for Industry" (Bioanalytical method validation. FDA, May 2001). All the parameters were validated considering the coefficient of variation (CV) of the ratio between the intensity of a certain analyte (A) and an internal standard (IS) over a variable number of repetitions (3-5) during several days (3-5). The methods were validated in terms of limit of detection, limit of quantification, precision, reproducibility, linearity, accuracy, recovery and selectivity. 


\subsection{Scope of the thesis}

The last decade has seen a vast array of medical discoveries and innovations. The word 'diagnostic' originated in the early $17^{\text {th }}$ century from the Greek expression 'hē diagnōstikē tekhnē' (the art of distinguishing) and diagnōstikos (able to distinguish). This thesis reports different aspects and applications of mass spectrometry as a tool 'able to distinguish' and characterize compounds in biological matrices in order to improve current methodologies for diagnostics.

Chapter 2 (Liquid Chromatography-Tandem Mass spectrometry in liquid clinical samples for doping controh discusses mass spectrometry coupled to liquid chromatography and applied to liquid biological samples (i.e. serum, blood, urine). Specifically, the development and applications of mass spectrometry to liquid biological samples is addressed in order to develop fast analytical procedures to quantify and qualify steroidal hormones in animal samples for doping control and screening applications. Three different protocols for the simultaneous identification and quantification of natural and synthetic steroids in serum, urine and blood from equines and bovines were developed. These were then applied to several real animal samples provided from veterinaries in Italy to monitor the abuse of these substances for doping purposes and/or to check the health status of the animals.

Chapter 3 (mass spectrometry imaging for lipid and metabolite analysis in fresh-frozen tissues) connects results from LC-MS and MSI by discussing matrix-assisted laser desorption/ionization (MALDI) mass spectrometry imaging (MSI) to investigate the causes and systematic effects of a lentil extract (LE) used as a cholesterol-lowering treatment. MALDI-MSI was used to investigate spatial modifications in the lipidic composition and cholesterol levels in the brain, liver and intestines as well as bile acids in the liver and intestine of rats treated with LE. This project originated from a collaboration 
with the University of Camerino (Italy) and is a follow-up study of a paper developed by Micioni Di Bonaventura et al. [45].

Chapter 4 (FFPE tissue-based quality control for MALDI-MSI analysis of tryptic peptides) tackles one of the current challenges related to MSI: the lack of a proper quality control. This chapter explores suitable quality controls for MSI analysis of clinical cohorts, which rely on the analysis of multiple samples over time. The tested quality control strategy was applied to characterize and predict pathological response to neoadjuvant treatment in breast cancer patients.

Finally, Chapter 5 (Tissue classification by Rapid Evaporative Ionization Mass Spectrometry [REIMS]: comparison between a diathermic knife and $\mathrm{CO}_{2}$ Laser sampling on classification performances) discusses an innovative mass spectrometry technique able to instantaneously ( $<3$ seconds) identify tissues sampled in ambient conditions. It explores the benefits and drawbacks of coupling a laser scalpel and a diathermic knife with a REIMS-TOF for real-time sample qualification/classification. This project also investigates the possibility to build a unique model for tissue qualification for both handpieces and to improve the classification rate of the proposed model by introducing a tissue-specific peak list-based model. 



\section{Chapter 2}

Liquid Chromatography-Tandem Mass spectrometry in liquid

clinical samples for doping control

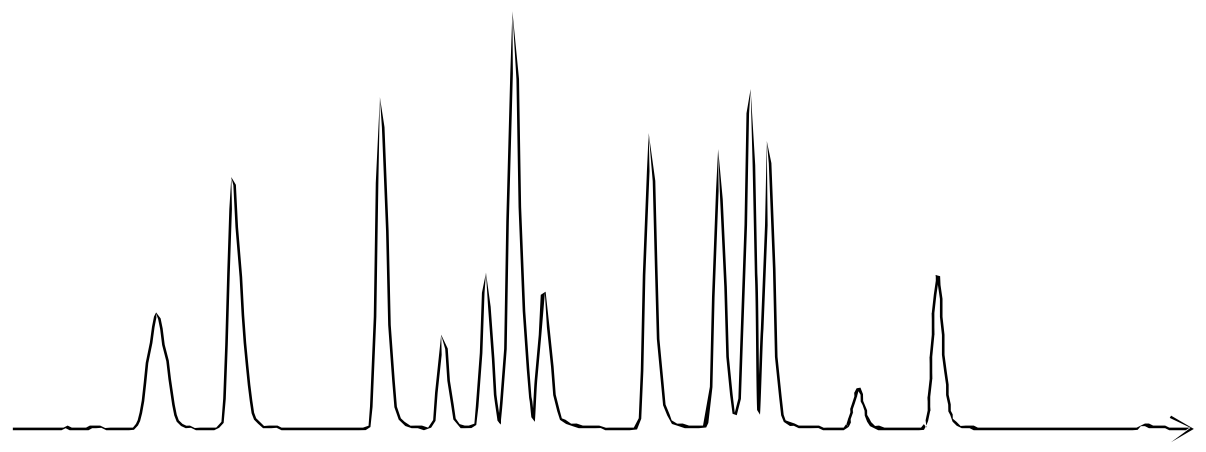

\section{Adapted from:}

Development and application of a UHPLC-MS/MS method for the simultaneous determination of 17 steroidal hormones in equine serum

Michele Genangeli, Giovanni Caprioli, Manuela Cortese, Fulvio Laus, Mara Matteucci, Riccardo Petrelli, Massimo Ricciutelli, Gianni Sagratini, Stefano Sartori, Sauro Vittori.

Journal of Mass Spectrometry 2016 .52(1): p. 22-29. DOI 10.1002/jms.3896

Qualification and quantification of 17 endogenous and exogenous steroidal hormones in equine and bovine blood for doping control with UHPLC-MS/MS: a new method.

Michele Genangeli, Giovanni Caprioli, Manuela Cortese, Riccardo Petrelli, Massimo Ricciutelli, Gianni Sagratini, Stefano Sartori, Sauro Vittori Journal of Chromatography A 2019 submitted

Simultaneous quantitation of 9 anabolic and natural steroidal hormones in equine urine by UHPLC-MS|MS.

Michele Genangeli, Giovanni Caprioli, Manuela Cortese, Fulvio Laus, Riccardo Petrelli, Massimo Ricciutelli, Gianni Sagratini, Stefano Sartori, Sauro Vittori

Journal of Chromatography B 2019 2019.1117: p. 36-40. DOI 10.1016/j.jchromb.2019.04.002 


\section{Liquid Chromatography-Tandem Mass spectrometry in liquid clinical samples for doping control}

\subsection{Abstract}

This chapter reports new, fast and simple analytical methods able to simultaneously identify and quantify endogenous and exogenous steroidal hormones and metabolites in biological matrices (e.g. serum, blood and urine) using a UHPLC-MS/MS. Once developed, the methods were fully validated according to the guidelines from the Guidance for Industries in terms of linearity, precision, accuracy, recovery, matrix effect and specificity. The methods were then applied to external biological samples.

\subsection{Introduction}

The earliest records of doping in sport come from the Ancient Olympics games when athletes are reported to have taken figs to improve their performances [46].

In "human" sport, a generally unified approach is taken where the rules and regulation of doping for the majority of elite sports events held in any country are governed by the World Anti-Doping Agency (WADA). As for humans, doping substances are largely used in competitions where animals are required as for in horseracing. In contrast of what exists for humans, horses and other competitions involving animals do not have a single organization regulating doping control programs, where anti-doping test and thresholds are generally different for each country or continent [47].

In equine sports, prohibited substances include not only doping agents that can be used to alter the performance of the animal, but also legitimate therapeutic substances that may be needed to ensure that the horse is healthy and fit to perform.

The International Federation of Horseracing Authorities (IFHA) was the first federation providing clear guidelines or recommended best practices for 
horseracing, precisely through the publication of the "International Agreement on Breeding Racing and Wagering" (IABRW). Due to the fact that doping substances may either be exogenous or endogenous in the animal, The Article 6A, Clause 14 of the IABRW document states that: 'International thresholds can only be adopted for: substances endogenous to the horse; substances arising from plants traditionally grazed or harvested as equine feed; substances in equine feed arising from contamination during cultivation, processing or treatment, storage or transportation'.

At present, there are ten substances with international threads holds (Table 1) in either urine or plasma or both. In addition to these, some authorities may have established thresholds for their own specific substances, and these thresholds can also be referred to as regulatory limits or reporting levels. The latter is specific to a particular racing authority whereas the former is applicable internationally in all relevant signatory countries [47]. 
Table 1 - International thresholds in Article 6A of the International Agreement on Breeding, Racing and Wagering (IABRW) published by the International Federation of Horseracing Authorities (IFHA)

\begin{tabular}{|c|c|}
\hline Threshold name & Threshold \\
\hline Arsenic & $0.3 \mu \mathrm{g}$ total arsenic per milliliter in urine \\
\hline Boldenone & $\begin{array}{l}0.015 \mu \mathrm{g} \text { free and conjugated boldenone per } \\
\text { milliliter in urine from male horses (other than } \\
\text { geldings) }\end{array}$ \\
\hline Carbon dioxide & $\begin{array}{l}36 \text { millimoles available carbon dioxide per liter in } \\
\text { plasma }\end{array}$ \\
\hline Dimethyl sulfoxide & $\begin{array}{l}15 \mu \mathrm{g} \text { dimethyl sulfoxide per milliliter in urine, or } \\
1 \mathrm{\mu g} \text { dimethyl sulfoxide per milliliter in plasma }\end{array}$ \\
\hline Estradiol in male horses (other than geldings) & $\begin{array}{l}0.045 \mu \mathrm{g} \text { free and glucuroconjugated } 5 \mathrm{a} \text {-estrane- } \\
3 \beta, 17 \mathrm{a} \text {-diol per milliliter in urine }\end{array}$ \\
\hline Hydrocortisone & $1 \mu \mathrm{g}$ hydrocortisone per milliliter in urine \\
\hline Methoxytyramine & $\begin{array}{l}4 \mu \mathrm{g} \text { free and conjugated 3-methoxytyramine per } \\
\text { milliliter in urine }\end{array}$ \\
\hline Salicylic acid & $\begin{array}{l}750 \mu \mathrm{g} \text { salicylic acid per milliliter in urine, or } 6.5 \mu \mathrm{g} \\
\text { salicylic acid per milliliter in plasma }\end{array}$ \\
\hline Testosterone & $\begin{array}{l}0.02 \mu \mathrm{g} \text { free and conjugated testosterone per } \\
\text { milliliter in urine from geldings, or } 0.055 \mu \mathrm{g} \text { free and } \\
\text { conjugated testosterone per milliliter in urine from } \\
\text { fillies and mares (unless in foal) }\end{array}$ \\
\hline Theobromine & $\begin{array}{l}2 \mu \mathrm{g} \text { theobromine per milliliter in urine, or } 0.3 \mu \mathrm{g} \\
\text { theobromine per milliliter in plasma }\end{array}$ \\
\hline \multicolumn{2}{|c|}{$\begin{array}{l}\text { When, at the screening stage, the free and glucuroconjugated } 5 a \text {-estrane- } 3 \beta, 17 a \text {-diol exceeds the free and } \\
\text { glucuroconjugated } 5,10 \text {-estrone- } 3 \beta, 17 a \text {-diol in the urine. }\end{array}$} \\
\hline
\end{tabular}

Apart from carbon dioxide, dimethyl sulfoxide, salicylic acid and theobromine, testosterone is still the only steroids regulated in plasma, and its

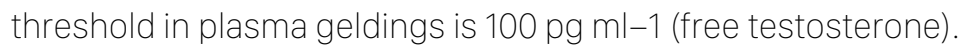

Steroids are a large class of compounds deriving from cholesterol that play a critical role in transmitting a vast array of biological signals in the organism[48]. Their functions can be broadly grouped into several categories: reproduction and sexual differentiation, development and growth, maintenance of the internal environment, and regulation of metabolism and nutrient supply [48]. Doping control in equine sports poses different challenges compared to those in human sports. In the latter, doping offences are committed almost without exception to improve performance. In equine sports, both performance enhancing and performance impairing substances (or methods) may be used in order to manipulate the outcome of the competition. This may be more 
predominant in horse racing where the potential gain from betting on other horses may outweigh the prize money from winning [47]. These substances are not harmless, because they may cause some pathologies like lung bleeding, hepatotoxicity, cardiac hypertrophy, tendinitis and articulation problems, and cancer, which could lead to stroke and death [49]. As reported from Kavitha et al., the followings are the adverse effects of anabolic steroids by topic: cardiovascular, endocrine and metabolic, gastrointestinal, genitourinary, hematologic and oncologic, neuromuscular and skeletal, neuropsychiatric, dermatologic and renal.

For the time being, many analytical procedures existing in literature for steroids are self-made analysis, often too complex or too long and expensive to be replicated in external clinical laboratories. Moreover, these methods are often based on immunoassay analysis, with poor sensitivity and high possibility of false negative and false positive response [50-54]. Lastly, it clearly arise that the majority of methods for steroids analysis focused firstly on human serum, and secondly on the concomitant help of HPLC-MS/MS (high performance liquid chromatography-tandem mass spectrometry), that is the ideal technique due to its high specificity and sensitivity [48, 55-65]. Huang et al. (2008) developed a method to determine six sexual steroid hormones in urine matrix by stir bar sorptive extraction (SBSE) coupled to high performance liquid chromatography (HPLC)-diode array detector (DAD); Magnisali et al (2008) used GC-MS (gas chromatography-mass spectrometry) for analyzing six steroids in serum of neonates [66]; Caron et al. (2015) reported a gas chromatography-tandem mass spectrometry (GC-MS/MS) method for the simultaneous quantification of ten endogenous steroids in serum from men, premenopausal and postmenopausal women [48]. Buttler et al. (2015) described the simultaneous measurement of 3 steroids, namely testosterone, androstenedione, dehydroepiandrosterone (DHEA) using ID (isotope dilution)-LC-MS/MS [67]; Ray et al. (2015) used LC-MS/MS combined with ion mobility spectrometry for the analysis of 5 endogenous steroids [61]; Ke et al. (2014) analyzed seven steroidal compounds by using UHPLC-MS/MS (with Q-trap like mass analyzer) [64]; Peitzsch et al. (2015) analyzed simultaneously 15 adrenal steroids in LC-MS/MS; in all cases the matrix was 
human serum [68]. Guan et al. (2005), analyzed eight major anabolic steroids in equine plasma [57], whereas Kaabia et al. (2013) analyzed a high number (20) of steroid esters in bovine and equine serum and plasma, following a long and time consuming procedure, i.e. solid phase extraction (SPE) before analysis with UHPLC-ESI-MS/MS [62]. Genye et al. developed a method to detect and analyze 13 steroids in human urine using a LC-quadrupole-Orbitrap Mass spectrometer [69]. Tajudheen et al. studied the separation of 2 anabolic substance using a reversed phase chiral chromatography approach [70]. Brian et al. published an article regarding novel liquid chromatography-tandem mass spectrometry methods for measuring steroids. Youwen et al. focused on the separation of 16 testosterone and nandrolone esters in equine plasma [71]. Colton et al. developed a method for fast screening of anabolic steroids in horse urine [72]. Voelker et al. developed a method for two anabolic steroids and UV detection [73]. Wang et al. developed a method for metabolites of stanozolol [74]. Baglai et al. published a paper for anabolic steroids in bovine urine which requires a two dimension LC analysis [75]. Pitarch-Motellón et al. developed a method able to detect four anabolic steroids in urine [76].

Here the necessity to develop, validate and commercialize new sensitive, robust and reproducible analytical methodologies that can quantify and qualify several endogenous and exogenous compounds in a single sample.

The following methodologies were developed for several steroids trying to keep them fast, easy (simple sample handling and sample preparation) and reproducible.

The proposed methodologies will be turned into "Ready to use Kits" and sold to external laboratories. 


\subsection{Experimental}

\subsubsection{Materials and standards}

All detected compounds used in this chapter, including the internal standard, were ordered from Sigma-Aldrich (Milan, Italy) with a purity > 99\%. Individual stock solutions of steroid hormones and metabolites were prepared by dissolving $1 \mathrm{mg}$ of each compound in $1 \mathrm{ml}$ of HPLC-grade methanol. The standard working solutions were obtained by diluting the stock solution at the concentration needed with methanol. HPLC-grade acetonitrile and methanol were purchased from Carlo Erba (Milano, Italy). HPLC-grade formic acid (99\%) was obtained from Merck (Darmstadt, Germany). Deionized water ( $>18 \mathrm{M} \Omega \mathrm{cm}$ resistivity) was purified using a Milli-Q SP Reagent Water System (Millipore, Bedford, MA, USA). All solvents and solutions were filtered through a 0.2 mm nylon membrane filter from Minisart RC 4, Sartorium Stedim (Goettingen, Germany) before transferring them into injection vials. Sterile glass tubes for blood collection were purchased from Becton-Dickinson (Franklin Lakes, NJ, USA). The analytical procedures were carried out in polypropylene vials, test tubes and plastic centrifuge tubes in order to preserve the concentration and stability of the hormones. The glass has demonstrated to interfere with those molecules as reported in the section "Evaluation of the stability of steroids in glass and plastic" (results and discussion and Table 10).

\subsubsection{Sample collection}

Equine and bovine blood, as well as equine serum and urine used to develop the methods, were collected from male and female healthy horses provided by the university veterinary hospital in Matelica (MC). The blood was stored in vials with EDTA or Lithium heparin. In order to obtain the serum, some blood was allowed to clot and centrifuged. An aliquot of serum from each horse was refrigerated at $-20^{\circ} \mathrm{C}$ and stored to be used as real sample. Blood used to validate the second analytical method was obtained from several veterinary hospitals from south of Italy, stored at $-4^{\circ} \mathrm{C}$ if analyzed within two days or stored at $-20^{\circ} \mathrm{C}$ if analyzed after two days. 


\subsubsection{Preparation of Steroids free matrices}

Serum $(50 \mathrm{ml})$ and $1 \mathrm{~g}$ of charcoal were stirred under magnetic agitation overnight in order to create a steroid free serum used in all validation steps (except for recovery studies and matrix effect performed in normal serum), as reported by Magnisali et al. [66]. The solution was centrifuged at $4000 \mathrm{rpm}$ for 20 minutes in plastic test tube and the supernatant was centrifuged two more times at 14000 rpm for 20 minutes in plastic centrifuge tubes in order to clean the serum from the charcoal residues. After these steps, the serum appears light yellow and clear. The purified serum was stored at $4^{\circ} \mathrm{C}$ and used to set-up the method and perform full validation (except for recovery studies and matrix effect, performed in normal serum).

Blood (50 ml) was kept under magnetic agitation overnight, with $1 \mathrm{~g}$ of charcoal to create steroids-free blood used in the development and validation steps. As reported by Genangeli et al. the solution was then settled for 10 minutes, and the clear blood without visual residues of charcoal was transferred into a clean $50 \mathrm{ml}$ plastic test tube [66]. To obtain clean blood from the charcoal residues, this last step was repeated for additional time or until the no residues of charcoal were evident on the bottom of the test tube. The hormone-free blood was stored at $-20^{\circ}$.

A total of $500 \mu \mathrm{L}$ of blood was denatured using a solution made of methanol $(\mathrm{MeOH})$ and $5 \mathrm{~g} / \mathrm{L}$ of zinc sulfate (ZnSO4) and internal standard (testosterone d3, 1000ppb) in Eppendorf tubes. The deproteinizing solution was prepared leaving the methanol and zinc sulfate under magnetic stirring overnight and then filtering the solution with a chapter filter. The solution was then agitated with vortex for approximately $1 \mathrm{~min}$ to avoid the formation of blood clotting. The solution was centrifuged for $15 \mathrm{~min}$ at $13000 \mathrm{rpm}$, and the liquid 
transferred into polypropylene test tubes. The obtained solution was evaporated under nitrogen gas flow, then reconstituted using $50 \mu \mathrm{\mu l}$ of $25 \%$ methanol and centrifuged again for 10 min at 13000rpm; finally, it was pipetted in high-recovery vials and injected in the UHPLC-ESI-MS/MS system.

Urine $(50 \mathrm{ml})$ was stirred with activated charcoal in order to obtain a matrix free of any steroid and stored at $-4^{\circ} \mathrm{C}$. The purification was performed according to the paper from Genangeli et al.[49]. The solution was then settled for 10 minutes, and the clear urine, without visual residues of charcoal, was transferred into a clean $50 \mathrm{ml}$ plastic test tube. In order to obtain clean urine from the charcoal residues, this last step was repeated for additional time or until the no residues of charcoal were present on the bottom of the test tube. All the validation steps were performed using the previously mentioned purified urine.

\subsubsection{Sample preparation and internal standards}

Serum: $400 \mu \mathrm{L}$ of serum (normal or steroids free) were transferred into a plastic centrifuge tubes, deproteinized using $1 \mathrm{ml}$ of deproteinizing solution (acetonitrile) and vortexed for 1 minute. The deproteinized solution was centrifugated at 14000 rpm for 15 minutes, then the supernatant is transferred into polypropylene test tube.

Three deuterated internal standards were used in order to increase the robustness of the method. The standards were added before deproteinization step at the concentration of: $250 \mathrm{ng} / \mathrm{ml} \mathrm{d}_{7}$-aldosterone, $100 \mathrm{ng} / \mathrm{ml} \mathrm{d}_{3}$-testosterone, 1000 $\mathrm{ng} / \mathrm{ml} \mathrm{d}_{4}$-cortisol. D D $_{3}$-testosterone was used as internal standard for TESTO, ANDD, DHEAS, DHEA, ESTRO, ANDRO, DHT and ESTRA, $d_{4}$-cortisol for 11-DOC, 17-OH-PROG, PRE, CORT, 17-OH-PRE, $d_{7}$-aldosterone for ALDO, COCO, PRO, 11-DCC.

The supernatant was evaporated using nitrogen gas flow. The dried samples were restored using $50 \mu$ of $25 \%$ methanol, transferred in plastic centrifuge tubes and 
centrifuged at 14000 rpm for 15 minutes. The supernatant was transferred in high recovery vials and injected in the UHPLC-ESI-MS/MS system.

Blood: A total of $500 \mu \mathrm{L}$ of blood was denatured using a solution made of methanol $(\mathrm{MeOH})$ and $5 \mathrm{~g} / \mathrm{L}$ of zinc sulfate $(\mathrm{ZnSO} 4)$ and internal standard (testosterone d3, 1000ppb) in Eppendorf tubes. The deproteinizing solution was prepared leaving the methanol and zinc sulfate under magnetic stirring overnight and then filtering the solution with a paper filter. The solution was then agitated with vortex for approximately 1 min to avoid the formation of blood clotting. The solution was centrifuged for 15 min at 13000 rpm, and the liquid transferred into polypropylene test tubes. The obtained solution was evaporated under nitrogen gas flow, then reconstituted using $50 \mu \mathrm{l}$ of $25 \%$ methanol and centrifuged again for 10 min at 13000rpm; finally, it was pipetted in high-recovery vials and injected in the UHPLC-ESI-MS/MS system.

One deuterated internal standard was introduced as a control to increase the robustness of the method. The standard was added prior to the deproteinization step at the concentration of 1000 ppb

Urine: A solution of Aldosterone D4 (internal standard, 1 $\mu \mathrm{g} / \mathrm{l}$ ) was added to $400 \mu \mathrm{L}$ of steroids-free urine. The mixture was evaporated under nitrogen gas flow. Dry samples were mixed with $50 \mu$ l of $25 \%$ methanol in water, centrifuged again for 9 min at 14000 rpm, transferred in high-recovery vials and injected in the UHPLC-ESI-MS/MS system.

\subsubsection{Liquid chromatography tandem mass spectrometry (UHPLC-ESI- MS/MS)}

UHPLC-ESI-MS/MS studies were performed using an Agilent 1290 Infinity series and a Triple Quadrupole 6420 from Agilent Technology (Santa 
Clara, CA) equipped with an ESI source operating in negative and positive ionization mode. The separation of analytes was achieved on a Zorbax RRHD C18 analytical column (50 x 2.10 mm i.d., $1.8 \mu \mathrm{m})$ from Agilent (USA). The mobile phase for UHPLCMS/MS analyses was a mixture of water (A) and acetonitrile (B) both containing $0.1 \%$ formic acid at $0.6 \mathrm{ml} / \mathrm{min}$ with a gradient elution different for each method:

Serum $=0 \min 15 \%$ B, $3.2 \min 42 \%$ B, $4.5 \min 42 \%$ B, 7 min $90 \% B, 9 \min 90 \%$, $9.50 \mathrm{~min} .15 \% \mathrm{~B}$, and kept at $15 \% \mathrm{~B}$ until the end of the run (11 min). The injection volume was $5 \mu$, performed with auto-sampler. The temperature of the column was $10^{\circ} \mathrm{C}$. 
Table 2 - Ultra-high-performance liquid chromatography-tandem mass spectrometry acquisition parameters (multiple reaction monitoring mode) used for the analysis of steroidal hormones and metabolites in blood

\begin{tabular}{|c|c|c|c|c|c|c|c|c|}
\hline $\begin{array}{l}\text { 무 } \\
\text { ㅇ } \\
\text { ํ } \\
\text { ㅇ }\end{array}$ & 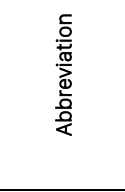 & 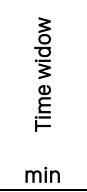 & 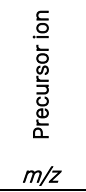 & $\begin{array}{l}\complement \\
\stackrel{0}{0} \\
\stackrel{0}{0} \\
\text { 음 } \\
m / z\end{array}$ & 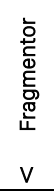 & 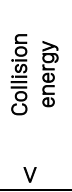 & 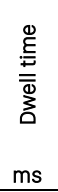 & 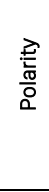 \\
\hline Cortisol & CORT & $1.3-2.5$ & 363.01 & 121.1 & 136 & 24 & 350 & POS \\
\hline- & - & - & - & 327.2 & 136 & 12 & - & - \\
\hline Aldosterone & ALDO & $1.3-2.5$ & 361.41 & 343.2 & 116 & 16 & 350 & POS \\
\hline- & - & - & - & 315.2 & 116 & 20 & - & - \\
\hline 11-Deoxycortisol & $11-\mathrm{DOC}$ & $2.5-3.3$ & 347.51 & 109.1 & 141 & 32 & 200 & POS \\
\hline- & - & - & - & 97.2 & 141 & 28 & - & - \\
\hline Corticosterone & CoCo & $2.5-3.3$ & 347.01 & 329.2 & 111 & 12 & 200 & POS \\
\hline- & - & - & - & 121.1 & 111 & 28 & - & - \\
\hline $\begin{array}{l}\text { Dehydroepiandrosterone } \\
\text { sulfate }\end{array}$ & DHEAS & $2.5-3.3$ & 366.99 & 97 & 165 & 32 & 200 & NEG \\
\hline- & - & - & - & 80 & 165 & 50 & - & - \\
\hline 17-OH-progesterone & $\begin{array}{l}\text { 17-OH- } \\
\text { PROG }\end{array}$ & $3.3-4.5$ & 331.01 & 313.2 & 102 & 4 & 100 & POS \\
\hline- & - & - & - & 57.2 & 102 & 24 & - & - \\
\hline 11-Deoxycorticosterone & $11-D C C$ & $3.3-4.5$ & 331.01 & 97.1 & 117 & 20 & 100 & POS \\
\hline- & - & - & - & 109.1 & 117 & 32 & - & - \\
\hline 17-OH-pregnenolone & 17-OH-PRE & 3.3-4.5 & 297.1 & 105.1 & 150 & 36 & 100 & POS \\
\hline- & - & - & - & 91.2 & 150 & 48 & - & - \\
\hline Dehydroepiandrosterone & DHEA & $3.3-4.5$ & 289.01 & 271.2 & 101 & 4 & 100 & POS \\
\hline- & - & - & - & 253.2 & 101 & 4 & - & - \\
\hline Testosterone & TESTO & $3.3-4.5$ & 289.01 & 97.1 & 131 & 20 & 100 & POS \\
\hline- & - & - & - & 109.1 & 131 & 28 & - & - \\
\hline Androstenedione & ANDD & $3.3-4.5$ & 287.01 & 97.1 & 131 & 24 & 100 & POS \\
\hline- & - & - & - & 109.1 & 131 & 24 & - & - \\
\hline Estrone & ESTRO & $3.3-4.5$ & 271.01 & 253.1 & 92 & 8 & 100 & POS \\
\hline- & - & - & - & 157.1 & 92 & 20 & - & - \\
\hline Estradiol & ESTRA & $3.3-4.5$ & 255.01 & 159.1 & 102 & 16 & 100 & POS \\
\hline- & - & - & - & 133.1 & 102 & 20 & - & - \\
\hline Androsterone & ANDRO & $4.5-5.6$ & 291.41 & 273.2 & 78 & 4 & 350 & POS \\
\hline- & - & - & - & 255.2 & 78 & 12 & - & - \\
\hline Dihydrotestosterone & DHT & $4.5-5.6$ & 273.1 & 255.3 & 159 & 15 & 350 & POS \\
\hline- & - & - & - & 147.0 & 159 & 16 & - & - \\
\hline Progesterone & PRO & $5.6-7.0$ & 315.01 & 97.1 & 126 & 24 & 400 & POS \\
\hline- & - & - & - & 109.1 & 126 & 24 & - & - \\
\hline Pregnenolone & PRE & $5.6-7.0$ & 299.01 & 281.2 & 111 & 8 & 400 & POS \\
\hline- & - & - & - & 105.0 & 111 & 40 & - & - \\
\hline d7-Aldosterone & d7-ALDO & $1.3-2.5$ & 368.3 & 350 & 135 & 15 & 350 & POS \\
\hline d4-Cortisol & d4-CORT & $1.3-2.5$ & 367.01 & 121 & 135 & 25 & 350 & POS \\
\hline d3-Testosterone & d3-TESTO & $3.3-4.5$ & 292 & 97 & 135 & 25 & 100 & POS \\
\hline
\end{tabular}

Blood $=0$ min 15\% B, 2.5 min 25\% B, 5 min 35\% B, 7 min 50\%, 9 min 90\% $B$ and 11 min 15\% B, kept at 15\% B until end of the run (15 min). Five $\mu$ l of samples were injected with an auto-sampler. The column was kept at $20^{\circ} \mathrm{C}$. 
Table 3 - Ultra-high-oerformance liquid chromatography-tandem mass spectrometry acquisition parameters (multiple reaction monitoring mode) used for the analysis of steroidal hormones and metabolites in Blood.

\begin{tabular}{|c|c|c|c|c|c|c|c|}
\hline compound & $\begin{array}{l}\text { 음 } \\
\stackrel{0}{\frac{\pi}{2}} \\
\text { d } \\
\frac{0}{0} \\
\frac{0}{0}\end{array}$ & $\begin{array}{c}3 \\
\frac{3}{0} \\
\frac{0}{3} \\
\pm \\
\stackrel{\Xi}{E} \\
\min \end{array}$ & 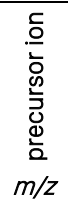 & 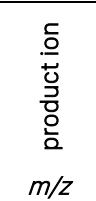 & 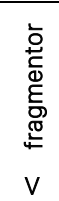 & 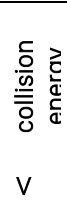 & 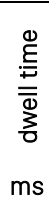 \\
\hline $\begin{array}{c}\text { dexamethasone sodium } \\
\text { phosphate }\end{array}$ & Desa-NA-P & $2.0-3.7$ & $\begin{array}{c}473 . \\
11\end{array}$ & $\begin{array}{l}435.2 \\
355.2\end{array}$ & 97 & 8 & 200 \\
\hline cortisol & CORT & $2.0-3.7$ & $\begin{array}{c}363 . \\
01\end{array}$ & $\begin{array}{l}121.1 \\
327.2\end{array}$ & 136 & 24 & 200 \\
\hline aldosterone & ALDO & $2.0-3.7$ & $\begin{array}{c}361 . \\
41\end{array}$ & $\begin{array}{l}343.2 \\
315.2\end{array}$ & 116 & 16 & 200 \\
\hline pregnenolone & PRE & $2.0-3.7$ & $\begin{array}{c}361 . \\
41\end{array}$ & $\begin{array}{l}343.2 \\
105.0\end{array}$ & 87 & 4 & 200 \\
\hline methylprednisolone & ME - PRE & $3.7-5.5$ & $\begin{array}{c}375 . \\
01\end{array}$ & $\begin{array}{l}357.2 \\
323.2\end{array}$ & 92 & 4 & 180 \\
\hline 11-deoxycortisol & $11-\mathrm{DOC}$ & $3.7-5.5$ & $\begin{array}{c}347 . \\
51\end{array}$ & $\begin{array}{c}109.10 \\
97.2\end{array}$ & 141 & 32 & 180 \\
\hline corticosterone & $\mathrm{COCO}$ & $3.7-5.5$ & $\begin{array}{c}347 . \\
01\end{array}$ & $\begin{array}{l}329.2 \\
329.2\end{array}$ & 111 & 12 & 180 \\
\hline stanozolol & STA & $3.7-5.5$ & $\begin{array}{c}329 . \\
51\end{array}$ & $\begin{array}{l}81.10 \\
95.10\end{array}$ & 170 & 50 & 180 \\
\hline boldenone & $\mathrm{BOL}$ & $3.7-5.5$ & $\begin{array}{c}287 . \\
41\end{array}$ & $\begin{array}{r}121.00 \\
135.00\end{array}$ & 107 & 24 & 180 \\
\hline nandrolone & NAN & $3.7-5.5$ & $\begin{array}{c}275 . \\
10\end{array}$ & $\begin{array}{l}109.10 \\
82.90\end{array}$ & 100 & 28 & 180 \\
\hline $\begin{array}{l}\text { dexamethasone } \\
\text { isonicotinate }\end{array}$ & DESA - ISO & $5.5-6.8$ & $\begin{array}{c}498 . \\
61\end{array}$ & $\begin{array}{l}47.20 \\
124.0\end{array}$ & 121 & 8 & 200 \\
\hline 11-deoxycorticosterone & $11-\mathrm{DCC}$ & $5.5-6.8$ & $\begin{array}{c}331 . \\
01\end{array}$ & $\begin{array}{l}97.10 \\
109.1\end{array}$ & 117 & 20 & 200 \\
\hline dihydrotestosterone & DHT & $6.8-9.0$ & $\begin{array}{c}273 . \\
10\end{array}$ & $\begin{array}{c}255.30 \\
147.0\end{array}$ & 159 & 15 & 200 \\
\hline testosterone & TESTO & $5.5-6.8$ & $\begin{array}{c}289 . \\
01\end{array}$ & $\begin{array}{l}97.10 \\
109.1\end{array}$ & 131 & 20 & 100 \\
\hline androstenedione & ANDD & $5.5-6.8$ & $\begin{array}{c}287 . \\
01\end{array}$ & $\begin{array}{l}97.10 \\
109.1\end{array}$ & 131 & 24 & 200 \\
\hline dehydroepiandrosterone & DHEA & $5.5-6.8$ & $\begin{array}{c}271 . \\
01\end{array}$ & $\begin{array}{c}253.10 \\
253.2\end{array}$ & 92 & 8 & 200 \\
\hline androsterone & ANDRO & $6.8-9.0$ & $\begin{array}{c}291 . \\
41\end{array}$ & $\begin{array}{c}273.20 \\
255.2\end{array}$ & 78 & 4 & 200 \\
\hline testosterone-d3 & d3 -TESTO & $5.5-6.8$ & $\begin{array}{c}292 . \\
00\end{array}$ & 97.00 & 135 & 25 & 120 \\
\hline
\end{tabular}

Urine $=0$ min 15\% B, 4,5 min 55\% B, 7 min 90\% B, 8 min 90\%B, 10 min 15\%B. Five $\mu \mathrm{l}$ of samples were injected with an autosampler. The column was kept at a temperature of $20^{\circ} \mathrm{C}$. 


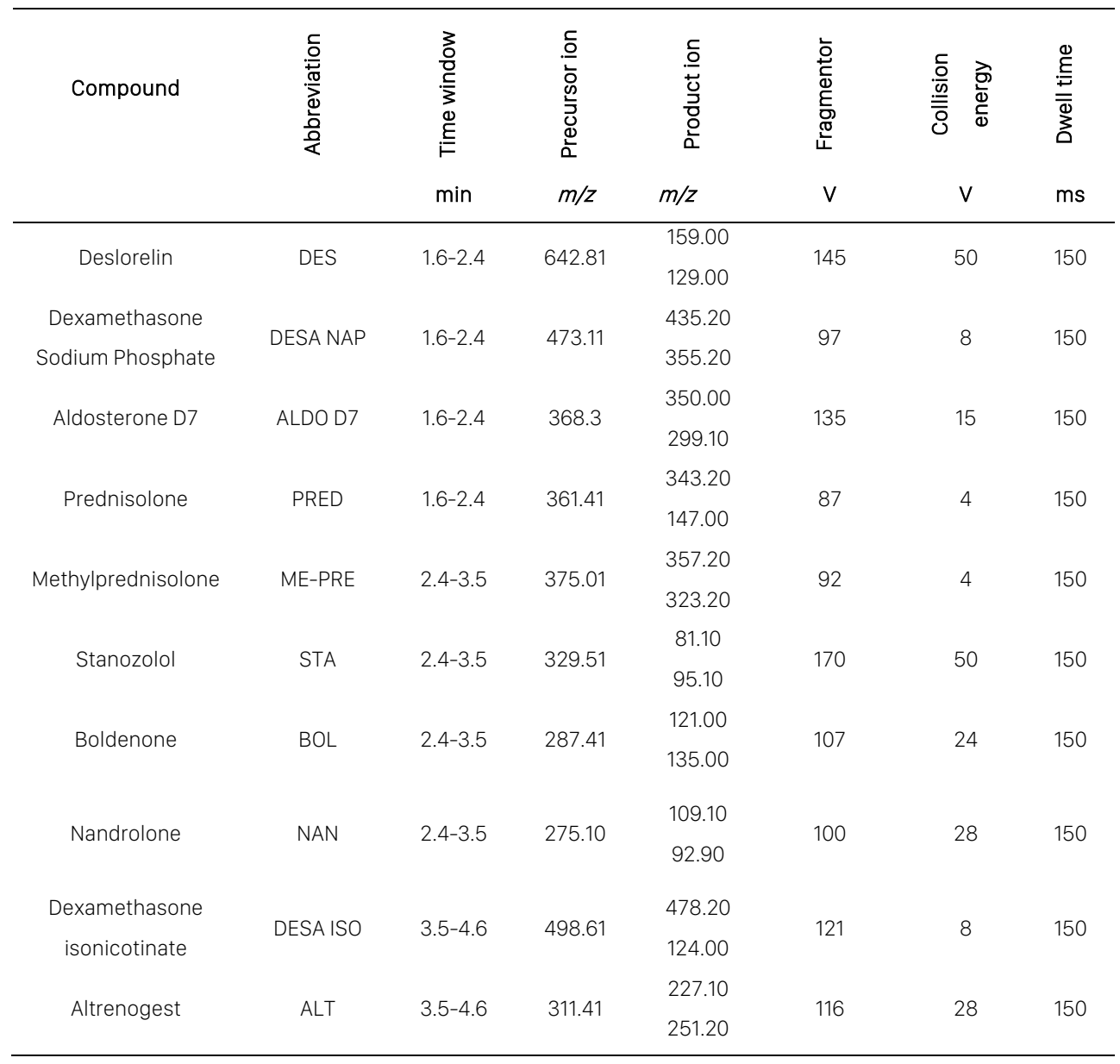

The temperature of the drying gas in the ionization source was $300{ }^{\circ} \mathrm{C}$. The gas flow was $12 \mathrm{l} / \mathrm{min}$, the nebulizer pressure was 40 psi and the capillary voltage was $4000 \mathrm{~V}$ (negative and positive). Detection was performed in the "multiple reaction monitoring" (MRM) mode dividing the run time in seven segments as reported in-Table 3-Table 4. The most abundant product ion was used for quantification, and the rest of the product ions were used for qualification. The monitored compounds, abbreviation, selected ion transition and the settings of the mass analyzer are reported in Table 2. 


\subsubsection{Calibration standards and controls}

In order to validate the method, different concentrations were used, starting from the LOQ (lowest one), to C1 (low concentration), CM (Medium concentration), C2 (high concentration), CU (upper concentration) (low range standard curve, 5 points) and four additional "upper" concentrations (U1-U2-U3-U4) for high range standard curve, for a total of 9 points. Table 5 reports the value of all the concentrations chosen for the calibration curve and the validation steps for the different compounds.

Table 5 - Concentration values used for the validation of the method for each analyte

\begin{tabular}{|c|c|c|c|c|c|c|c|c|c|c|}
\hline 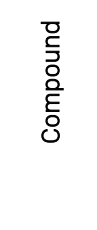 & LOD & LOQ & $\mathrm{C} 1$ & 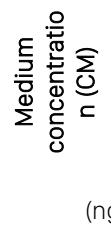 & -1) & $\mathrm{CU}$ & U1 & U2 & U3 & U4 \\
\hline \multicolumn{11}{|c|}{ Serum } \\
\hline CORT & 0.002 & 0.0055 & 2.7 & 27.5 & 55 & 40 & 220 & 330 & 440 & 550 \\
\hline ALDO & 0.03 & 0.055 & 0.275 & 2.75 & 5.5 & 11 & 22 & 33 & 44 & 55 \\
\hline 11-DOC & 0.2 & 0.55 & 2.75 & 27.5 & 55 & 110 & 220 & 330 & 440 & 550 \\
\hline CoCo & 0.02 & 0.05 & 0.25 & 2.5 & 5 & 10 & 20 & 30 & 40 & 50 \\
\hline DHEAS & 0.2 & 0.55 & 2.75 & 27.5 & 55 & 110 & 220 & 330 & 440 & 550 \\
\hline $\begin{array}{c}17-\mathrm{OH}- \\
\text { PROG }\end{array}$ & 0.2 & 0.55 & 2.75 & 5.5 & 11 & 22 & 44 & 66 & 88 & 110 \\
\hline $11-D C C$ & 0.2 & 0.55 & 2.75 & 27.5 & 55 & 110 & 220 & 330 & 440 & 550 \\
\hline $\begin{array}{c}\text { 17-OH- } \\
\text { PRE }\end{array}$ & 0.3 & 1.1 & 2.2 & 22 & 44 & 88 & 176 & 264 & 352 & 440 \\
\hline DHEA & 0.5 & 1.1 & 5.5 & 55 & 110 & 220 & 440 & 660 & 880 & 1100 \\
\hline TESTO & 0.02 & 0.05 & 0.275 & 2.75 & 5.5 & 11 & 22 & 33 & 44 & 55 \\
\hline ANDD & 0.025 & 0.05 & 0.25 & 2.5 & 5 & 10 & 20 & 30 & 40 & 50 \\
\hline ESTRO & 0.05 & 0.11 & 0.55 & 5.5 & 11 & 22 & 44 & 66 & 88 & 110 \\
\hline ESTRA & 0.02 & 0.055 & 0.275 & 2.75 & 5.5 & 11 & 22 & 33 & 44 & 55 \\
\hline ANDRO & 0.05 & 0.11 & 0.55 & 5.5 & 11 & 22 & 44 & 66 & 88 & 110 \\
\hline DHT & 0.02 & 0.055 & 0.275 & 2.75 & 5.5 & 11 & 22 & 33 & 44 & 55 \\
\hline PROG & 0.2 & 0.55 & 2.75 & 27.5 & 55 & 110 & 220 & 330 & 440 & 550 \\
\hline
\end{tabular}




\begin{tabular}{|c|c|c|c|c|c|c|c|c|c|c|}
\hline PRE & 2 & 5.5 & 11 & 110 & 220 & 440 & 880 & 1320 & 1760 & 2200 \\
\hline \multicolumn{11}{|c|}{ Blood } \\
\hline $\begin{array}{l}\text { DESA- } \\
\text { NA-P }\end{array}$ & 0.333 & 1.00 & 10 & 50 & 100 & 500 & 1000 & - & - & - \\
\hline COR & 0.183 & 0.55 & 11 & 55 & 110 & 550 & 1100 & - & - & - \\
\hline ALDO & 0.183 & 0.55 & 11 & 55 & 110 & 550 & 1100 & - & - & - \\
\hline PRE & 0.033 & 0.10 & 10 & 50 & 100 & 500 & 1000 & - & - & - \\
\hline ME - PRE & 0.067 & 0.20 & 20 & 100 & 200 & 1000 & 2000 & - & - & - \\
\hline $11-\mathrm{DOC}$ & 0.037 & 0.11 & 11 & 55 & 110 & 550 & 1100 & - & - & - \\
\hline $\mathrm{COCO}$ & 0.167 & 0.50 & 10 & 50 & 100 & 500 & 1000 & - & - & - \\
\hline STA & 0.033 & 0.10 & 10 & 50 & 100 & 500 & 1000 & - & - & - \\
\hline $\mathrm{BOL}$ & 0.167 & 0.50 & 10 & 50 & 100 & 500 & 1000 & - & - & - \\
\hline \multicolumn{11}{|c|}{ Urine } \\
\hline DES & 2.50 & 5.00 & 10 & 20 & 60 & 80 & 100 & 200 & 300 & 400 \\
\hline $\begin{array}{l}\text { DESA } \\
\text { NAP }\end{array}$ & 0.50 & 1.00 & 5 & 10 & 30 & 40 & 100 & 200 & 300 & 400 \\
\hline PRED & 0.25 & 0.50 & 5 & 10 & 30 & 40 & 100 & 200 & 300 & 400 \\
\hline ME-PRE & 0.25 & 0.50 & 5 & 10 & 30 & 40 & 100 & 200 & 300 & 400 \\
\hline STA & 0.10 & 0.25 & 5 & 10 & 30 & 40 & 100 & 200 & 300 & 400 \\
\hline $\mathrm{BOL}$ & 0.10 & 0.25 & 5 & 10 & 30 & 40 & 100 & 200 & 300 & 400 \\
\hline NAN & 0.50 & 1.00 & 5 & 10 & 30 & 40 & 100 & 200 & 300 & 400 \\
\hline DESA ISO & 0.10 & 0.25 & 5 & 10 & 30 & 40 & 100 & 200 & 300 & 400 \\
\hline ALT & 0.10 & 0.25 & 5 & 10 & 30 & 40 & 100 & 200 & 300 & 400 \\
\hline
\end{tabular}




\subsection{Development and application of a UHPLC-MS/MS method for the simultaneous determination of 17 steroidal hormones in equine serum}

The aim of our work was to set-up a new UHPLC-MS/MS triple quadrupole method to detect and quantify seventeen hormones and metabolites in equine serum. Quantifiable hormones with the proposed method are: Pregnenolone, 17-OHPregnenolone, Progesterone, 17-OH-Progesterone, Androsterone, Androstenedione, DHEA, DHEAS, Testosterone, Cortisol, Corticosterone, Aldosterone, 11-Deoxycortisol, 11-Deoxycorticosterone, Dihydrotestosterone, Estrone, Estradiol. Three deuterated hormones (Cortisol-D4, Aldosterone-D7, Testosterone-D3) have been used as internal standards in order to set a more accurate and precise procedure (Table 2, Table 3 and Table 4). Most of the compounds chosen in the current method are often different from those reported in literature and not usually included in analytical methods $[57,62]$. The procedure is fast, sample preparation is easy, the method is sensitive, accurate and robust and it could bring a remarkable saving of time and money with respect to previously reported methods. After full method validation, this procedure has been successfully applied to the analysis of serum samples from different kind of horses (mare, stallion and gelding).

\subsubsection{Results and Discussion}

\section{Optimization of chromatographic conditions}

Different solvents were tested and the mixture of water-formic acid $0.1 \%$, (mobile phase A) and acetonitrile-formic acid 0.1\% (mobile phase B) was the ideal combination for the analysis and separation of this complex mixture of standards. Use of methanol as mobile phase $B$, or only water as mobile phase $A$, leads to worse separation among peaks and a lower sensitivity. Moreover, considering the different and wide degrees of polarity of the seventeen steroids, the gradient elution was used to achieve the best separation. Under the optimum gradient conditions, as reported in paragraph "Liquid chromatography tandem mass spectrometry", the baseline separation of all peaks of those compounds was achieved. In our experiments we found that this time of final conditioning (at least $1.5 \mathrm{~min}$.) is indispensable for 
reproducibility of retention times of the monitored analytes. However, other gradient conditions caused a poor separation of some peaks or overlapping of analytes. Additionally, the flow rate at $0.6 \mathrm{ml} \mathrm{min}^{-1}$ seems to be the best for our purpose, instead flow rate of $0.4-$ and $0.5-\mathrm{ml} \mathrm{min}^{-1}$ caused a longer analytical time without improvement, and flow rate of $0.7 \mathrm{ml} \mathrm{min}{ }^{-1}$ caused a worst separation of all steroids.

\section{Mass spectrometry conditions and ESI (+) and ESI (-) MS spectra}

Mobile phase composition and additives may have a significant influence on the response of the solute with ESI source. Thus, two different mobile phase compositions (i.e. acetronitrile-water and methanol-water, both containing formic acid) were tested and the first one was chosen for the analysis, as the response was significantly higher than methanol-water; also, chromatography separation and resolution of peaks were excellent. Even using an aprotic organic solvent plus water, the mobile phases provided enough protons in positive mode sufficient for steroidal hormones protonation without affecting the abundance of ions formed [77]. According to literature, the use of formic acid as additives is often a good choice in positive mode to increase the response of target compounds; in fact, in our case it improved both ionization and chromatographic separation/resolution of peaks. For most of the monitored compounds, the precursor ion was the protonated molecule $[\mathrm{M}+\mathrm{H}]^{+}$ in positive polarity and the deprotonated molecule $[\mathrm{M}-\mathrm{H}]^{-}$for the only analyte (DHEAS) monitored in negative polarity. On the other hand, only for ESTRA, DHT and PRE, the precursor ion in positive polarity was the $\left[\mathrm{M}+\mathrm{H}-\mathrm{H}_{2} \mathrm{O}\right]^{+}$as reported by Shao et al. Abundant $[\mathrm{M}+\mathrm{H}]^{+}$or $[\mathrm{M}-\mathrm{H}]^{-}$ions are always desired for sensitivity of qualitative and quantitative methods. Conversely, pseudo-molecular ions (in particular solvent adduct) are undesirable since they decrease abundance of $[\mathrm{M}+\mathrm{H}]^{+}$ions. Even if methanol is, for this aspect, prefeared over acetonitrile as it 
showed lower proton affinity, and thus it has lower tendency to form solvent adduct ions, we similarly obtained selective ionization using acetonitrile.

The tandem MS product ion spectrum in the triple quadrupole mass spectrometer is the result of a one-step process in which several product ions are formed and in which it is difficult to ascertain the relationship of the precursor and product ions. Compared with the fragmentation in other mass analyzers (ion trap, Orbitrap, Q-TOF), a much higher abundance of product ions formed by recyclization cleavages is found in the product ion spectra in the triple quadrupole. This occurs for 8 steroids, whose quantitative product ion formed is not easily decipherable with a low resolution mass analyzer like triple quadrupole. On the other side, for ALDO, CoCo, 17$\mathrm{OH} P R O G, \mathrm{DHEA}, \mathrm{ESTRO}$ and $\mathrm{ANDRO}$, the quantitative product ion is the $\left[\mathrm{M}-\mathrm{H}_{2} \mathrm{O}+\mathrm{H}\right]^{+}$ and for ESTRA, DHT and PRE the main product ion formed was the $\left[\mathrm{M}-2 \mathrm{H}_{2} \mathrm{O}+\mathrm{H}\right]^{+}$, as reported in literature [78].

\section{Method validation}

The analytical characteristics of the developed method were investigated, including linearity, limit of detection (LODS), limits of quantification (LOQs), accuracy and precision, recovery and matrix effect, to evaluate their efficacy for application on the analysis of steroids hormones in equine samples.

Concentration values used for the validation steps have been chosen according to the LOQ of each compound (Table 5).

\section{Linearity}

As mentioned before, two types of standard curves were prepared by analyzing spiked hormones free serum samples (see paragraph "Calibration standards and controls") at different concentration levels. The calibration curves were calculated with all the concentrations from the LOQ to the CU, (low range standard curve, 5 
points) form data obtained during a 3-day validation and using three repetitions of each value every day. The obtained $\mathrm{R}^{2}$ are higher than $0.9990 \%$ (Data not shown).

High range standard curve was calculated with all the nine concentrations from the $L O Q$ to the $U 4$, in order to test the upper limit of linearity and to assess linearity in a wider dynamic range. Data were obtained from three repetitions of all concentration, and the results were expressed in terms of coefficient of linear regression $\left(R^{2}\right)$, slope and intercept of the twentyseven curves obtained. The developed method displayed good linearity, being the correlation coefficients range of the analyzed compounds calibration curves in the range 0.9922-0.9986 (Data not shown).

The limits of detection (LODs) and limits of quantification (LOQs) found in the study were in the range of $0.002-2 \mathrm{ng} \mathrm{ml}^{-1}$ and $0.0055-5.5 \mathrm{ng} \mathrm{ml}^{-1}$, respectively. The LOD and LOQ obtained in the present study are similar or even lower than the limits described in literature; even if most of the compounds chosen in the current method are often different from those included in analytical method available in literature. Moreover, LOQ for testosterone is equal to $0.05 \mathrm{ng} \mathrm{ml}^{-1}, 50 \%$ lower with respect to $0.1 \mathrm{ng} \mathrm{ml}^{-1}$ (or $100 \mathrm{pg} \mathrm{ml}^{-1}$ ) that is the admitted limit in plasma geldings [79].

\section{Precision}

Precision is the closeness of agreement between independent test results obtained under stipulated conditions. It is usually specified in terms of standard deviation or relative standard deviation [80].

The precision (intra- and inter-day) was calculated with the LOQ, CM, CU and U3, from data obtained during a 3-day validation (Table 6). Each day, five repetitions of the three concentrations were tested and the precision results for each concentration were reported in term of CV\% (coefficient of variation). 
The coefficient of variation for all compounds were satisfactory and within the range 0.33-18.74\% (Table 6). The inter-day $(n=5)$ method precision was also satisfactory as expressed by the percent RSD values that were obtained. At the LOQ concentration, the percent RSD values were within the range 13.46-17.82\%, at the CM concentration the \% RSD values were $7.39-18.74 \%$, at the CU concentration the \% RSD values were 3.52-18.15\% and at the U3 concentration the \% RSD values were $0.33-9.21 \%$ for the 17 steroid hormones examined in this study (Table 6).

Table 6 - Intra-day and inter-day precision expressed in CV\%

\begin{tabular}{|c|c|c|c|c|c|c|c|c|}
\hline \multirow{2}{*}{$\begin{array}{l}\text { 을 } \\
\text { 응 } \\
\text { 읕 } \\
\text { 이 }\end{array}$} & \multicolumn{2}{|c|}{$\begin{array}{c}\text { Limit of } \\
\text { quantification }\end{array}$} & \multicolumn{2}{|c|}{$\begin{array}{c}\text { Medium } \\
\text { concentration }\end{array}$} & \multicolumn{2}{|c|}{$\mathrm{CU}$} & \multicolumn{2}{|c|}{ U3 } \\
\hline & $\begin{array}{l}\text { Intra-day } \\
(\mathrm{CV} \%)\end{array}$ & $\begin{array}{l}\text { Inter-day } \\
(\mathrm{CV} \%)\end{array}$ & $\begin{array}{l}\text { Intra-day } \\
(\mathrm{CV} \%)\end{array}$ & $\begin{array}{c}\text { Inter-day } \\
(\mathrm{CV} \%)\end{array}$ & $\begin{array}{c}\text { Intra-day } \\
(\mathrm{CV} \%)\end{array}$ & $\begin{array}{l}\text { Inter-day } \\
(\mathrm{CV} \%)\end{array}$ & $\begin{array}{l}\text { Intra-day } \\
(\mathrm{CV} \%)\end{array}$ & $\begin{array}{c}\text { Inter-day } \\
(\text { CV\%) }\end{array}$ \\
\hline CORT & 10.62 & 13.46 & 6.31 & 7.39 & 2.46 & 3.95 & 0.86 & 1.32 \\
\hline ALDO & 16.23 & 17.69 & 9.03 & 10.14 & 6.30 & 8.04 & 3.39 & 4.32 \\
\hline 11-DOC & 14.21 & 16.74 & 6.51 & 8.20 & 6.60 & 7.44 & 5.89 & 6.66 \\
\hline CoCo & 15.60 & 16.64 & 9.60 & 10.70 & 2.61 & 3.52 & 1.02 & 2.21 \\
\hline DHEAS & 15.69 & 16.67 & 14.50 & 17.60 & 6.14 & 18.15 & 3.09 & 7.93 \\
\hline $\begin{array}{l}\text { 17-OH- } \\
\text { PROG }\end{array}$ & 16.86 & 17.08 & 6.20 & 7.53 & 6.74 & 7.34 & 5.30 & 6.54 \\
\hline 11-DCC & 10.10 & 13.86 & 7.52 & 10.42 & 1.40 & 4.34 & 0.33 & 2.12 \\
\hline $\begin{array}{l}\text { 17-OH- } \\
\text { PRE }\end{array}$ & 13.01 & 14.24 & 5.31 & 8.91 & 2.98 & 5.06 & 1.02 & 4.03 \\
\hline DHEA & 15.59 & 17.10 & 11.35 & 16.42 & 3.54 & 17.80 & 1.87 & 6.78 \\
\hline TESTO & 17.11 & 17.82 & 6.68 & 14.52 & 5.31 & 7.94 & 2.10 & 5.65 \\
\hline ANDD & 17.19 & 17.57 & 9.30 & 18.74 & 7.69 & 9.45 & 4.32 & 6.22 \\
\hline ESTRO & 16.36 & 17.28 & 15.51 & 18.53 & 11.99 & 18.02 & 7.02 & 9.21 \\
\hline ESTRA & 11.52 & 17.38 & 10.91 & 16.65 & 6.16 & 14.83 & 4.22 & 5.54 \\
\hline ANDRO & 9.84 & 15.90 & 13.52 & 18.21 & 8.36 & 9.68 & 6.11 & 7.87 \\
\hline $\mathrm{DHT}$ & 13.97 & 17.05 & 9.52 & 18.39 & 4.49 & 8.29 & 2.02 & 3.09 \\
\hline PRO & 16.57 & 16.99 & 4.92 & 8.03 & 4.13 & 4.62 & 2.76 & 3.04 \\
\hline PRE & 13.73 & 16.33 & 8.61 & 8.99 & 6.13 & 6.38 & 4.12 & 5.12 \\
\hline
\end{tabular}




\section{Accuracy}

Accuracy is the closeness of agreement between a testresult and the accepted reference value of the property being measured [80].

The accuracy (intra- and inter-day) was calculated using the C1, C2 and U3 spiked concentrations, from the data obtained during a 3-day validation. The results are reported in Table 7 , and expressed in term of relative error $\%$. The percentage relative errors for all the analytes in intra-day experiments were satisfactory and within the range, 0.92-13.90\% (Table 7). The inter-day $(n=5)$ method accuracy was also satisfactory as expressed by the percent RSD values that were obtained: at the C1 concentration, the \% RE values were in the range 6.52-13.24\% at the C2 concentration, the \% RE values were $2.26-7.72 \%$ and at the U3 concentration, the \% RE values were 1.93-4.94\%.

Table 7 - Intra-day and inter-day accuracy expressed in RE\%

\begin{tabular}{|c|c|c|c|c|c|c|}
\hline \multirow{2}{*}{ 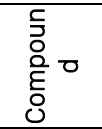 } & \multicolumn{2}{|c|}{ C1 } & \multicolumn{2}{|c|}{$\mathrm{C} 2$} & \multicolumn{2}{|c|}{ U3 } \\
\hline & $\begin{array}{l}\text { Intra-day } \\
\text { (RE\%) }\end{array}$ & $\begin{array}{l}\text { Inter-day } \\
\text { (RE\%) }\end{array}$ & $\begin{array}{c}\text { Intra-day } \\
\text { (RE\%) }\end{array}$ & $\begin{array}{l}\text { Inter-day } \\
\text { (RE\%) }\end{array}$ & $\begin{array}{c}\text { Intra-day } \\
\text { (RE\%) }\end{array}$ & $\begin{array}{c}\text { Inter-day } \\
\text { (RE\%) }\end{array}$ \\
\hline CORT & 6.73 & 6.94 & 1.92 & 2.41 & 1.76 & 1.93 \\
\hline ALDO & 10.90 & 11.46 & 4.44 & 4.81 & 2.34 & 3.83 \\
\hline $11-D O C$ & 9.42 & 9.77 & 3.55 & 4.72 & 2.55 & 2.73 \\
\hline CoCo & 7.63 & 10.21 & 6.46 & 7.25 & 3.41 & 4.24 \\
\hline DHEAS & 8.71 & 9.52 & 3.27 & 5.58 & 1.27 & 3.27 \\
\hline $\begin{array}{c}17-\mathrm{OH}- \\
\text { PROG }\end{array}$ & 10.92 & 12.03 & 4.38 & 5.77 & 2.39 & 3.79 \\
\hline 11-DCC & 5.92 & 6.52 & 1.99 & 2.26 & 0.92 & 2.20 \\
\hline $\begin{array}{c}\text { 17-OH- } \\
\text { PRE }\end{array}$ & 12.95 & 13.72 & 6.03 & 6.14 & 4.04 & 4.12 \\
\hline DHEA & 8.46 & 9.72 & 6.24 & 6.53 & 3.25 & 3.92 \\
\hline TESTO & 13.24 & 13.43 & 4.45 & 5.02 & 2.11 & 3.11 \\
\hline ANDD & 11.23 & 10.85 & 6.36 & 7.72 & 4.37 & 4.94 \\
\hline ESTRO & 12.62 & 13.90 & 5.37 & 7.31 & 3.38 & 3.65 \\
\hline ESTRA & 9.11 & 13.70 & 5.71 & 6.60 & 3.67 & 4.66 \\
\hline ANDRO & 8.41 & 9.48 & 5.21 & 5.62 & 4.16 & 4.67 \\
\hline DHT & 10.70 & 11.17 & 3.12 & 4.66 & 2.26 & 3.68 \\
\hline PRO & 9.52 & 10.72 & 4.03 & 4.34 & 2.02 & 2.85 \\
\hline PRE & 7.05 & 9.01 & 3.82 & 5.29 & 2.22 & 3.24 \\
\hline \multicolumn{7}{|c|}{$\mathrm{RE} \%$, relative error. } \\
\hline
\end{tabular}




\section{Recovery}

Recovery studies were performed by spiking normal equine serum with a mixture standard of the seventeen hormones. The recovery value was obtained using the following formula: $\left(\left(A_{s e}-A_{\text {sblank }}\right) / A_{\text {std }}\right) \times 100$, where $A_{\text {se }}$ is the area about the serum enriched with a low concentration (C1 and CM) of all the compounds, $A_{\text {blank }}$ is the area of analytes detected in the serum, $A_{\text {std }}$ is the area of a mixture standard of all the compounds dissolved in methanol. The recoveries obtained by spiking the matrix at the CM concentration were in the range of $91.05-97.66 \%$, with CV lower than 5.04\% (Table 8). Moreover, the recoveries at a concentration of C1 were in the range 85.6099.39\%, with CV lower than 8.37\% (Table 8).

Table 8 - Percent recovery and reproducibility at two fortification levels

\begin{tabular}{|c|c|c|c|c|}
\hline \multirow{2}{*}{ Compounds } & \multicolumn{2}{|c|}{$\mathrm{CM}$} & \multicolumn{2}{|c|}{$\mathrm{C} 1$} \\
\hline & Recovery $n=3$ & CV\% & Recovery $n=3$ & $\mathrm{CV} \%$ \\
\hline CORT & 96.64 & 1.36 & 91.03 & 4.22 \\
\hline ALDO & 95.09 & 0.94 & 89.64 & 5.80 \\
\hline $11-\mathrm{DOC}$ & 97.59 & 1.53 & 92.06 & 5.61 \\
\hline CoCo & 92.79 & 5.04 & 87.42 & 5.79 \\
\hline DHEAS & 92.51 & 1.96 & 95.79 & 3.72 \\
\hline 17-OH-PROG & 97.66 & 0.91 & 92.10 & 6.02 \\
\hline 11-DCC & 95.85 & 3.10 & 90.16 & 2.93 \\
\hline 17-OH-PRE & 91.84 & 2.05 & 86.58 & 5.23 \\
\hline DHEA & 96.03 & 3.08 & 90.56 & 6.44 \\
\hline TESTO & 95.69 & 0.78 & 91.99 & 6.07 \\
\hline ANDD & 95.70 & 1.06 & 99.39 & 6.16 \\
\hline ESTRO & 92.02 & 2.69 & 86.66 & 3.43 \\
\hline ESTRA & 93.53 & 1.01 & 88.09 & 5.24 \\
\hline ANDRO & 95.71 & 3.59 & 89.98 & 8.37 \\
\hline $\mathrm{DHT}$ & 91.05 & 1.08 & 85.60 & 5.72 \\
\hline PRO & 95.27 & 2.57 & 89.78 & 7.36 \\
\hline \multirow[t]{2}{*}{ PRE } & 93.09 & 0.65 & 87.74 & 4.44 \\
\hline & \multicolumn{3}{|c|}{ CV\%, coefficient of variation. } & \\
\hline
\end{tabular}

\section{Matrix effect}

Matrix effects can lead to either a reduced response (ion suppression) or an increased response (ion enhancement) of the mass spectrometry system [80]. These 
effects can severely compromise quantitative analysis of biological samples using LC-ESI-MS. In order to investigate the matrix effect, a post column infusion was performed. Post column infusion is one of the best techniques used to obtain qualitative information about matrix effects [81]. A methanolic mixture of all the compounds at CM concentration have been infused in the ESI using a micro pump and then an injection of extracted serum was performed. As shown in Figure 11, the signal remains constant for almost all the chromatographic time, except for a "valley" (signal suppression) at 7.5 minutes. All the compounds have a retention time shorter than 7 minutes, thus it is evident that the matrix does not have any suppression or enhancement effect in these analysis.

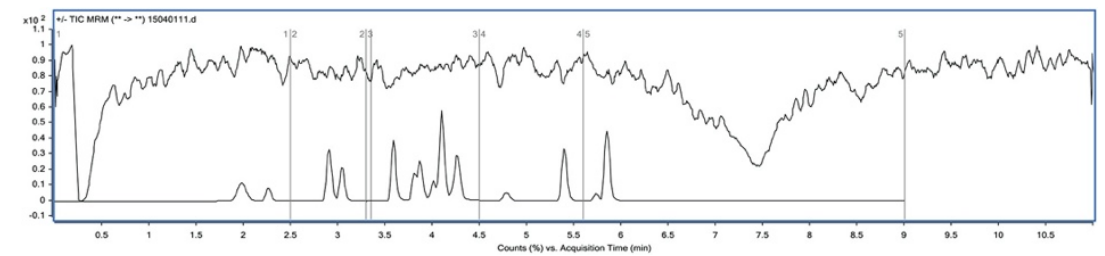

Figure 11 - Post-column infusion of steroids serum - free and a mixture standard of all the searched compounds in high-performance liquid chromatography-grade methanol.

\section{Specificity}

High specificity was achieved using tandem mass spectrometry. Both retention time stability and multiple precursor/product ion pairs were utilized to demonstrate the specificity of the method. Reproducibility of the chromatographic retention time for each compound was examined five times over a five day period ( $n=25)$. The retention times using this method were stable with RSD \% values $\leq 0.98 \%$.

Specific precursor/product ion transitions were identified for each steroids and the MRM transition with the most abundant product ion was selected for quantitation and the other product ion was selected for qualification (Table 1). 


\section{Application of the developed method to the analysis of equine serum.}

The high sensitivity and the quantitative aspect of the method permitted to detect most of steroids in four equine serum, i.e. two mare, one stallion and one gelding serum.

Only aldosterone, DHEAS, DHEA and estrone were not detected in any samples (Table 9). The main steroids found in the four samples were corticosterone (range 37.25-51.26 $\mathrm{ng} \mathrm{ml}^{-1}$ ) and cortisol (range 32.57-52.24 $\mathrm{ng} \mathrm{ml}^{-1}$ ), followed by 17-OHpregnenolone, dihydrotestosterone and pregnenolone. Stallion displayed the highest total steroids level (155.12 $\mathrm{ng} \mathrm{ml}^{-1}$ ), followed by gelding (133.68 $\mathrm{ng} \mathrm{ml}^{-1}$ ) and mare. Stallion serum displayed higher levels of $17-\mathrm{OH}$-pregnenolone and testosterone (27.24 and $4.80 \mathrm{ng} \mathrm{ml}^{-1}$ respectively) with respect to gelding (7.81 $\mathrm{ng} \mathrm{ml-1}-$ n.d.), but comparable amount of others steroids such as androstenedione, androsterone and pregnenolone. Level of testosterone in geldings is lower that LOQ (i.e. $50 \mathrm{pg} \mathrm{ml}^{-1}$ ), and thus lower than the threshold regulated in plasma (100 pg ml-1 of free testosterone) [79]. On the other side, the level found in stallion equine sample is quite high (4800 pg $\mathrm{ml}^{-1}$ ) but testosterone is still not regulated in stallion. Stallion and gelding showed higher level of cortisol, corticosterone and 17-OH-pregnenolone with respect to mare, but comparable levels of pregnenolone. Only in the two mare serum samples, we were able to detect estradiol (1.38-5.74 $\left.\mathrm{ng} \mathrm{ml}^{-1}\right)$ at conspicuous levels. 
Table 9 - Content of steroidal hormones in equine serum samplesa, expressed in $n g \mathrm{mt}^{-1}$

\begin{tabular}{ccccc}
\hline Analytes & Mare & Mare & Stallion & Gelding \\
\hline CORT & 32.57 & 36.14 & 50.76 & 52.24 \\
ALDO & n.d. & n.d. & n.d. & n.d. \\
11-DOC & n.d. & 0.25 & 0.25 & 0.08 \\
CoCo & 42.38 & 37.25 & 51.13 & 51.26 \\
DHEAS & n.d. & n.d. & n.d. & n.d. \\
17-OH-PROG & 0.04 & n.d. & 0.11 & 0.08 \\
11-DCC & 0.03 & n.d. & n.d. & 0.03 \\
17-OH-PRE & n.d. & 5.32 & 27.24 & 7.81 \\
DHEA & n.d. & n.d. & n.d. & n.d. \\
TESTO & 0.04 & 0.04 & 4.80 & n.d. \\
ANDD & n.d. & 0.18 & 0.30 & 0.17 \\
ESTRO & n.d. & n.d. & n.d. & n.d. \\
ESTRA & 1.38 & 5.74 & n.d. & n.d. \\
ANDRO & 0.26 & 4.05 & 2.02 & 0.91 \\
DHT & n.d. & 12.62 & 9.62 & 10.92 \\
PRO & 0.42 & 3.11 & 0.11 & 0.08 \\
PRE & 3.99 & 9.03 & 8.78 & 9.37 \\
Total & 81.10 & 113.72 & 155.12 & 133.68 \\
a Each sample was analyzed in triplicate. Percent RSDs in all cases were lower than 13.66\%. & & \\
& & b n.d., not detectable. & & \\
\hline & & & & \\
\hline
\end{tabular}




\subsubsection{Conclusions}

In this sub-chapter an ultra-high-performance liquid chromatography-tandem mass spectrometry (UHPLC-MS/MS) method has been developed, permitting the detection of 17 steroids in equine serum samples. The procedure is fast and intuitive, the sample preparation is easy, with deproteinization inside the vials followed by centrifugation and instrumental analysis. The present analytical method exhibited good performances in terms of specificity, sensitivity (LOQ in the range 0.0055-5.5 ng $\mathrm{ml}^{-1}$ ) and linearity. Another advantage of this developed analytical protocol is the simultaneous monitoring of a very large number of different hormones presenting various steroid substructures in a short time (11 minutes chromatographic run). Most of the compounds chosen in the current method are often different from those reported in literature and not usually included in analytical methods. The method permitted to detect most of steroid esters in equine serum. After full method validation, the procedure has been successfully applied to the analysis of equine serum samples (mare, gelding and stallion). The main steroids found in the four samples were corticosterone and cortisol, followed by 17-OH-pregnenolone, dihydrotestosterone and pregnenolone. Aldosterone, DHEAS, DHEA and estrone were detected in any samples. In conclusion, the present method allows identification and quantification of steroids and it could be used when fraudulent use is suspected in racing animals or in equine trade. 


\subsection{Qualification and quantification of 17 endogenous and exogenous steroidal hormones in equine and bovine blood for doping control with UHPLC-MS/MS: a new method.}

This sub-chapter reports a follow-up study of a method developed by Genangeli et al. for the simultaneous determination of steroids in Horse serum [49]. It is worth remember that endogenous and exogenous steroids (i.e. anabolic steroids, synthetic compounds derived from testosterone) are abused in animal-related sports, and they have a major role in regulating a wide number of endogenous signals in the organism $[82,83]$. Nowadays, competitions or events involving animals like cattle or horses are increasing in popularity. As previously reported from Genangeli et al. , only eleven compounds are present in the list of prohibited substances with international thresholds in both urine or plasma [79]. These substances can cause severe harm to the animal.

Quantitative and qualitative evaluation of steroidal hormones and their metabolites is crucial for the correct diagnosis and/or treatment of several diseases and conditionsin the animal [48]. At the moment, current analytical procedures regarding the matter are self-developed analysis, usually expensive, complicated or long and time-consuming to be replicated in clinical laboratories $[50,52,53,84]$.

This work aimed to set up a new UHPLC-tandem mass based method to detect and quantify seventeen hormones and metabolites in equine and bovine blood. Detectable and quantifiable compounds included in the developed method are as follows: Androsterone (AND), Androstenedione (ANDD), Dehydroepiandrosterone (DHEA), Testosterone (TEST), Cortisol (COR), Corticosterone (COCO), Aldosterone (ALDO), 11-deoxycortisol (11-DOC), 11deoxycorticosterone (11-DCC), Dihydrotestosterone (DHT), Nandrolone (NAN), Boldenone (BOL), Stanozolol (STA), Dexamethasone Sodium Phosphate (Desa $\mathrm{NaP}$ ), Dexamethasone isonicotinate, (Desa-Iso), Methylprednisolone (MePre) and Pregnenolone (PRE). One deuterated hormone (testosterone-D3) was used 
as an internal standard in order to make the analytical method more robust (Table 3). All the compounds included in this methodology are currently monitored in doping analysis, but none of the procedures reported in the literature is able to detect and quantify all of them simultaneously $[57,62]$. The proposed procedure is not timeconsuming with a well described and simple sample preparation. Additionally, the method resulted in being sensitive, accurate and robust after a full validation. Hence, it could bring faster and cheaper analysis easily applicable from any analytical laboratory. The proposed procedure was fully validated and applied to the analysis of blood samples from different kinds of animals (mares, stallions, geldings and cows).

\subsubsection{Results and discussion \\ Setup of the chromatographic and mass analyzer conditions}

After testing different chromatographic conditions, the best results were obtained with a solution of water and $0.1 \%$ of formic ( mobile phase $A$ ) and acetonitrile and $0.1 \%$ of formic acid (mobile phase B). The use of other solvents as mobile phase B led to a worse separation among peaks, and the usage of only water as mobile phase b showed a lower sensitivity.

Due to the different chemical structure of the analytes, several chromatographic-gradient conditions were tested. Complete baseline separation of all peaks while keeping the run as fast as possible, was achieved using the chromatographic condition listed in Table 3 and the section 'Liquid ChromatographyTandem Mass Spectrometry'. A final column conditioning was also found to be essential for reproducibility of the retention time of the monitored compounds. A flow rate at $0.6 \mathrm{ml} \mathrm{min}{ }^{-1}$ was the best option to achieve a good chromatographic separation in a short period of time. Different flow rates led to either a longer analytical time or an overlapping of peaks.

As for the optimization of the chromatographic conditions, the mobile phases were chosen accordingly to their influence in the ionization process occurring in the ESI source. The choice of acetonitrile and water with formic acid led to a significantly 
higher signal and ionization for all the compounds and also to a good chromatography separation and resolution of peaks [85, 86]. Additionally, papers in literature confirm that the addition of formic acid in a positive mode increases the response of target compounds $[77,86]$. In our case, we had an improvement of both ionization and chromatographic separation/resolution of peaks.

The precursor and daughter ion obtained injecting a standard solution of each compound are comparable with other methods found in the literature

\section{Evaluation of the stability of steroids in glass and plastic}

Several endogenous and exogenous steroids were tested for stability in glass and plastic. A standard concentration of 200 ppb of the compounds listed in Table 10was prepared, and an aliquot of the before mentioned mix was transferred into four plastic vials and four glass vials and stored at $-4 \mathrm{C}$. The first vial was immediately analysed and the other three were analysed over the following 3 days. As reported in Table 10, immediately after one day the concentration of the steroids stored in the glass test tube dropped, with a loss of $>94 \%$. 
Table 10 - Loss of compounds in plastic vs glass containers.

\begin{tabular}{ccccc}
\hline & \multicolumn{2}{c}{ DAY1 } & \multicolumn{2}{c}{ DAY 2 } \\
COMPOUND & $\begin{array}{c}\text { Loss in } \\
\text { Plastic (\%) }\end{array}$ & $\begin{array}{c}\text { Loss in } \\
\text { Glass }(\%)\end{array}$ & $\begin{array}{c}\text { Loss in } \\
\text { Plastic (\%) }\end{array}$ & $\begin{array}{c}\text { Loss in } \\
\text { Glass }(\%)\end{array}$ \\
\hline COR & $<1$ & 99.63 & $<2$ & $>99.90$ \\
ALDO & $<1$ & 94.29 & $<2$ & $>99.90$ \\
11 - DOC & $<1$ & 99.94 & $<2$ & $>99.90$ \\
COCO & $<1$ & 99.76 & $<2$ & $>99.90$ \\
$11-$ DCC & $<1$ & 99.88 & $<2$ & $>99.90$ \\
PRE & $<1$ & 99.97 & $<2$ & $>99.90$ \\
ANDRO & $<1$ & 99.52 & $<2$ & $>99.90$ \\
TESTO & $<1$ & 99.85 & $<2$ & $>99.90$ \\
ANDD & $<1$ & 99.83 & $<2$ & $>99.90$ \\
DHT & $<1$ & 99.12 & $<2$ & $>99.90$ \\
DHEA & $<1$ & 99.65 & $<2$ & $>99.90$ \\
\hline
\end{tabular}

\section{Method validation}

The proposed method was fully validated in terms of its analytical characteristics such as linearity, accuracy and precision, evaluation of the limit of detection (LOD) and limit of quantification (LOQ). Additionally, recovery and matrix were also investigated. The assessment of all these parameters is essential for the future application of the proposed method. All the concentration were developed starting from the LOQ of every compound (Table 5).

\section{Precision and Linearity}

Precision is known to be the closeness of agreement between independent test results obtained under stipulated conditions $[49,87]$. It is usually reported regarding standard deviation (SD) or relative standard deviation (RSD) $[49,87]$.

The precision (intra-day and inter-day) was calculated from data obtained during a three-day validation (Table 11) of five daily repetitions using four concentrations from the LOQ to the U1 (LOQ, CM, CU and U1). The outcome is 
expressed according to the coefficient of variation (CV\%). The $\mathrm{CV}$ resulted to be included in the range of $0.48-18.78 \%$ (Table 11). The inter-day precision $(n=5)$ expressed in relative standard deviation percent (RSD) was also satisfactory. The LOQ displayed RSD in the range of 10.86-18.37\%, the CM resulted in an RSD of $2.62-18.78 \%$, the CU showed an RSD of $3.52-18.40 \%$ and U1 had an RSD within $0.48-9.41 \%$ (Table 11).

To calculate the linearity of the proposed method, two calibration curves were created using all the concentrations between LOQ and CU (low-range standard curve, 5 points, 5-day validation) and all the concentrations between the LOQ and U1 (high-range standard curve, 5 points, 5-day validation). Highrange curve was used to test the linearity in a more extense dynamic range. Linearity is expressed as the coefficient of linear regression $\left(R^{2}\right)$, and it is higher than $0.99 \%$ (Table 11).

The LODs and LOQs for all compounds included in this analytical procedure displayed values in the range of 0. 0.023-1.833 and 0.069-5.5 ppb, respectively. These values are similar and sometimes lower when compared with a limit of detection and quantification reported in the literature $[57,62,71]$. Additionally, the steroids and metabolites included in this procedure were chosen due to their frequency of usage and because partially included in procedure already present in the literature. Moreover, LOQ for testosterone is equal to $0.05 \mathrm{ppb}$, twice lower concerning $0.1 \mathrm{ppb}$ (or $100 \mathrm{ppt}$ ), which is the threshold for plasma samples of young horses (geldings) [79]. 


\begin{tabular}{|c|c|c|c|c|c|c|c|c|c|}
\hline \multirow{2}{*}{ 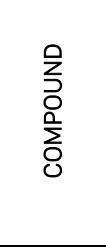 } & \multicolumn{2}{|c|}{ LOQ } & \multicolumn{2}{|c|}{$\begin{array}{c}\text { MEDIUM } \\
\text { CONCENTRATION }\end{array}$} & \multicolumn{2}{|c|}{$\mathrm{CU}$} & \multicolumn{2}{|c|}{ U1 } & \multirow{2}{*}{ 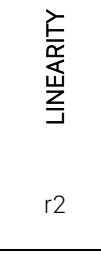 } \\
\hline & $\begin{array}{c}\text { intra- } \\
\text { day } \\
(\mathrm{cv} \%)\end{array}$ & $\begin{array}{c}\text { inter- } \\
\text { day } \\
\text { (cv\%) }\end{array}$ & $\begin{array}{l}\text { intra-day } \\
(\mathrm{cv} \%)\end{array}$ & $\begin{array}{c}\text { inter- } \\
\text { day } \\
(\mathrm{c} \vee \%)\end{array}$ & $\begin{array}{c}\text { intra- } \\
\text { day } \\
(\mathrm{cv} \%)\end{array}$ & $\begin{array}{c}\text { inter- } \\
\text { day } \\
(\mathrm{c} \%)\end{array}$ & $\begin{array}{c}\text { intra- } \\
\text { day } \\
(\mathrm{cv} \%)\end{array}$ & $\begin{array}{c}\text { inter- } \\
\text { day } \\
(\mathrm{cv} \%)\end{array}$ & \\
\hline $\begin{array}{l}\text { DESA- } \\
\text { NA-P }\end{array}$ & 10.86 & 14.16 & 6.74 & 7.58 & 2.62 & 4.12 & 1.02 & 1.48 & 0.99326 \\
\hline COR & 16.47 & 18.49 & 9.50 & 10.35 & 6.49 & 8.24 & 3.56 & 4.49 & 0.99939 \\
\hline ALDO & 14.45 & 17.52 & 6.95 & 8.40 & 6.79 & 7.63 & 6.07 & 6.85 & 0.99995 \\
\hline PRE & 15.84 & 17.41 & 10.07 & 10.91 & 2.78 & 3.69 & 1.18 & 2.37 & 0.99922 \\
\hline $\begin{array}{l}\text { ME - } \\
\text { PRE }\end{array}$ & 15.93 & 17.45 & 15.04 & 17.85 & 6.32 & 18.40 & 3.26 & 8.12 & 0.99912 \\
\hline $11-\mathrm{DOC}$ & 17.10 & 17.87 & 6.63 & 7.72 & 6.93 & 7.53 & 5.48 & 6.73 & 0.99954 \\
\hline COCO & 10.34 & 14.57 & 7.97 & 10.63 & 1.56 & 4.51 & 0.48 & 2.28 & 0.99991 \\
\hline STA & 13.25 & 14.95 & 5.73 & 9.11 & 3.15 & 5.24 & 1.18 & 4.20 & 0.99941 \\
\hline BOL & 15.83 & 17.89 & 11.85 & 16.66 & 3.71 & 18.05 & 2.03 & 6.97 & 0.99658 \\
\hline NAN & 17.35 & 18.62 & 7.12 & 14.75 & 5.49 & 8.13 & 2.26 & 5.83 & 0.99861 \\
\hline $\begin{array}{l}\text { DESA - } \\
\text { ISO }\end{array}$ & 17.43 & 18.37 & 9.77 & 18.99 & 7.88 & 9.65 & 4.49 & 6.41 & 0.99981 \\
\hline $11-\mathrm{DCC}$ & 16.60 & 18.07 & 16.06 & 18.78 & 12.21 & 18.27 & 7.21 & 9.41 & 0.99953 \\
\hline TESTO & 11.76 & 18.17 & 11.40 & 16.89 & 6.35 & 15.06 & 4.39 & 5.72 & 0.99841 \\
\hline ANDD & 10.08 & 16.66 & 14.04 & 18.46 & 8.56 & 9.88 & 6.29 & 8.06 & 0.99987 \\
\hline DHEA & 14.21 & 17.84 & 9.99 & 18.64 & 4.67 & 8.49 & 2.18 & 3.26 & 0.99970 \\
\hline ANDRO & 16.81 & 17.77 & 5.34 & 8.23 & 4.30 & 4.80 & 2.93 & 3.21 & 0.99940 \\
\hline DHT & 13.97 & 17.10 & 9.07 & 9.19 & 6.31 & 6.57 & 4.29 & 5.30 & 0.99997 \\
\hline
\end{tabular}

\section{Accuracy}

Accuracy is known to be the closeness of agreement between a test result and the accepted reference value of the property being measured $[49,88]$.

The intra and inter-day accuracy were calculated from the C1, C2 and U1 concentrations, from the data obtained during a three-day validation. The results are listed in Table 12 and expressed in terms of 'relative error percentage' (RE\%). The RE\% for all the analytes were within the range, $0.83-13.64 \%$ (Table 12). The inter-day ( $n=5)$ 
accuracy was also satisfactory. Precisely, at the C1 concentration, the RE\% values were in the range $5.22-13.64 \%$, at the C2 concentration, the RE\% values were $1.07-7.12 \%$, and at the U1 concentration, the RE\% values were $0.83-4.19 \%$.

Table 12 - Intra-day and inter-day accuracy expressed in RE\%

\begin{tabular}{|c|c|c|c|c|c|c|}
\hline \multirow[b]{2}{*}{ COMPOUND } & \multicolumn{2}{|c|}{$\mathrm{C} 1$} & \multicolumn{2}{|c|}{$\mathrm{C} 2$} & \multicolumn{2}{|c|}{ U1 } \\
\hline & $\begin{array}{c}\text { Intra-day } \\
\text { (RE\%) }\end{array}$ & $\begin{array}{c}\text { Inter-day } \\
\text { (RE \%) }\end{array}$ & $\begin{array}{c}\text { Intra-day } \\
\text { (RE \%) }\end{array}$ & $\begin{array}{c}\text { Inter-day } \\
\text { (RE \%) }\end{array}$ & $\begin{array}{c}\text { Intra-day } \\
\text { (RE \%) }\end{array}$ & $\begin{array}{c}\text { Inter-day } \\
\text { (RE \%) }\end{array}$ \\
\hline DESA-NA-P & 6.08 & 6.30 & 1.23 & 1.52 & 0.83 & 1.01 \\
\hline COR & 10.48 & 11.07 & 3.66 & 4.05 & 1.44 & 3.02 \\
\hline ALDO & 8.92 & 9.29 & 2.72 & 3.95 & 1.66 & 1.85 \\
\hline PRE & 7.03 & 9.75 & 5.79 & 6.63 & 2.57 & 3.45 \\
\hline$M E-P R E$ & 8.17 & 9.02 & 2.42 & 4.86 & 1.31 & 2.42 \\
\hline $11-\mathrm{DOC}$ & 10.50 & 11.67 & 3.60 & 5.06 & 1.50 & 2.97 \\
\hline coco & 5.22 & 5.85 & 1.07 & 1.36 & 1.65 & 1.29 \\
\hline STA & 12.64 & 13.45 & 5.34 & 5.45 & 3.24 & 3.32 \\
\hline BOL & 7.90 & 9.23 & 5.56 & 5.87 & 2.40 & 3.11 \\
\hline NAN & 12.95 & 13.15 & 3.67 & 4.27 & 1.20 & 2.26 \\
\hline DESA - ISO & 10.83 & 10.43 & 5.69 & 7.12 & 3.59 & 4.19 \\
\hline $11-\mathrm{DCC}$ & 12.29 & 13.64 & 4.64 & 6.69 & 2.54 & 2.83 \\
\hline TESTO & 8.59 & 13.43 & 5.00 & 5.94 & 2.85 & 3.89 \\
\hline ANDD & 7.85 & 8.98 & 4.47 & 4.90 & 3.36 & 3.90 \\
\hline DHEA & 10.27 & 10.76 & 2.27 & 3.89 & 1.36 & 2.86 \\
\hline ANDRO & 9.02 & 10.29 & 3.23 & 3.55 & 1.10 & 1.98 \\
\hline DHT & 6.41 & 8.48 & 3.00 & 4.56 & 1.32 & 2.39 \\
\hline
\end{tabular}

\section{Recovery}

Recovery was studied by spiking clean equine and bovine blood with a mixture standard of the 17 hormones. The recovery value was obtained using the following formula: $\left(\left(A_{\text {se }}-A_{\text {blank }}\right) / A_{\text {std }}\right) \times 100$, where $A_{\text {se }}$ is the area about the serum enriched with a low concentration (C1 and CM) of all compounds, $A_{\text {blank }}$ is the area of analytes detected in the serum and $A_{\text {std }}$ is the area of a mixture standard of all the compounds dissolved in methanol. The recoveries obtained by spiking the matrix at the CM concentration were in the range of 91.0598.32\%, with a CV lower than 5.04\% (Table 13). Moreover, the recoveries at a concentration of C1 were in the range 86.75-96.39\%, with a CV lower than 8.21\% (Table 13). 
Table 13 - Percent recovery and reproducibility at two fortification levels

\begin{tabular}{ccccc}
\hline COMPOUND & Recovery $n=3$ & CV & Recovery $n=3$ & CV\% \\
\hline DESA-NA-P & 97.66 & 0.91 & 91.05 & 3.10 \\
COR & 96.64 & 1.36 & 89.77 & 5.66 \\
ALDO & 95.09 & 0.94 & 92.13 & 3.58 \\
PRE & 93.09 & 0.65 & 90.15 & 2.49 \\
ME - PRE & 98.32 & 2.01 & 95.61 & 6.69 \\
11- DOC & 97.59 & 1.53 & 93.33 & 4.25 \\
COCO & 92.79 & 5.04 & 86.75 & 6.98 \\
STA & 95.06 & 0.99 & 90.91 & 3.36 \\
BOL & 96.21 & 1.21 & 93.28 & 5.75 \\
NAN & 98.32 & 3.45 & 96.39 & 8.21 \\
DESA - ISO & 97.16 & 4.00 & 94.45 & 6.74 \\
11 - DCC & 95.85 & 3.10 & 92.76 & 4.69 \\
TESTO & 95.69 & 0.78 & 93.44 & 2.57 \\
ANDD & 95.70 & 1.06 & 93.31 & 3.99 \\
DHEA & 96.03 & 3.08 & 90.18 & 6.85 \\
ANDRO & 95.71 & 3.59 & 93.64 & 7.69 \\
DHT & 91.05 & 1.08 & 88.54 & 5.50 \\
\hline
\end{tabular}

\section{Matrix effect}

Matrix is often responsible for a reduced or an increased signal/ionization (ion suppression/enhancement) in mass spectrometry [80]. These effects can strongly affect and compromise the quality and reproducibility of biological samples when injected and studied with LC-ESI-MS. To test the matrix effect, we performed a test known as "post-column infusion". This test, according to the literature, is one of the most effective techniques used to obtain qualitative information about matrix effects, [81]. A methanol mixture of all the compounds at the medium concentration (CM) was injected in the ESI source using a micropump. Simultaneously, an injection of purified and deproteinized blood was performed. As shown in Figure 12, the signal is constant for almost all the chromatographic time, with the exception for a signal suppression at $9.5 \mathrm{~min}$. All the compounds have a retention time shorter than $9.5 \mathrm{~min}$; hence, the matrix does not cause ion suppression or enhancement effects in our method. 


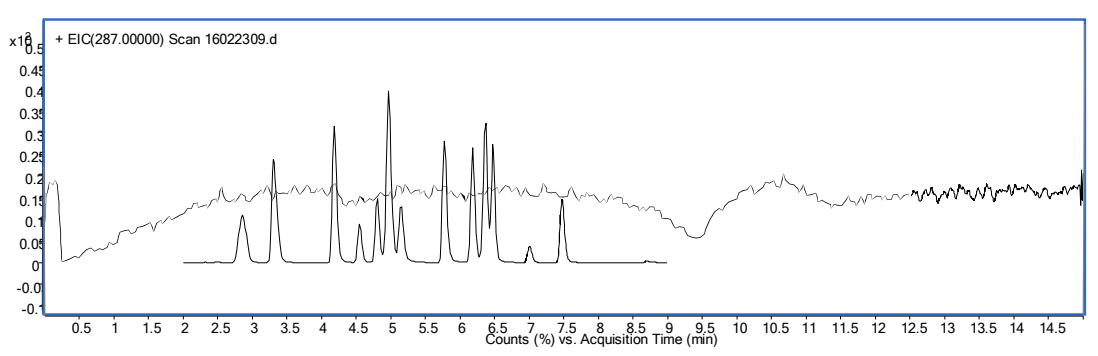

Figure 12 - Post column infusion of steroids serum-free and a mixture standard of all searched compounds in high-performance liquid chromatography-grade methanol.

\section{Specificity}

In order to quantify the specificity of the proposed method, we controlled the retention time of parent/daughter ions for all the analytes over time. For each compound, we examined the chromatographic retention time regarding reproducibility, for five times over a five-day period $(n=25)$. The RSD regarding the retention time was stable with an average percent value $\leq 0.97 \%$.

Specific parent/daughter ion transitions were identified for each steroid, and the MRM transitions with the most abundant product ion were selected for quantitation, and the other product ion was selected for qualification (Table 3). High specificity was achieved.

\section{Application and testing of the developed method to equine and bovine blood}

The above described method was successfully applied to thirty-three samples provided from four control agencies located in the south of Italy (Groups A, B, C, D). The high sensitivity and the quantitative aspect of the proposed method allowed the detection of most of the compound in the equine and bovine blood samples. The analysis was performed in blind, and due to privacy, we do not know if samples were bovine or equine. Due to the blind analysis and the thresholds changing between gender, species and age of the animals, we could not compare our results with international guidelines. 
A total of thirty-three animals were analyzed in triplicate, percent RSDs in all cases were lower than $13.66 \%$. Moreover, the mean values of the analytes found in the various samples are reported in Table A 1.

Only androsterone and DHEA were not detected in any samples. The main compound found in the four groups of samples was cortisol (range 0.44-10.00 ppb), followed by corticosterone, androstenedione and dexamethasone isonicotinate.

Overall, the level of exogenous steroids is below the thresholds from different guidelines and papers in the literature (Pregnenolone, Stanozolol and Nandrolone < $1 \mathrm{ppb}$, Boldenone < 15ppb, testosterone < 20ppb for geldings in plasma and $<55 \mathrm{ppb}$ for mares and fillies not in foal, $[47,89,90])$.

None of the samples showed levels of exogenous substances above 1 ppb, with the exception of ME-PRE in group C. Some animals showed traces of some of the exogenous steroids. In particular, DESA-NA-P is present only in the group A. PRE is present only in two samples belonging to group A. BOL was present only in 3 samples and not in group C. NAN was present in three samples belonging to groups A and D.

\subsubsection{Conclusions}

A new UHPLC-MS/MS method was developed, permitting the detection of 17 endogenous and exogenous anabolic substances in equine and bovine blood samples. The sample preparation is time-saving, fast and intuitive. The validation of the proposed method showed excellent performance regarding specificity, sensitivity (LOQ in the range of 0.069-5.5 ppb) and linearity. With the proposed method it is possible to simultaneously monitor, quantify and qualify a large number of steroids in a short time presenting various steroid substructures in a short time $(15 \mathrm{~min}$ chromatographic run) from either equine or bovine blood. Most of the compounds in the current method are different from methods reported in the literature but largely adopted to modify the condition of the considered animals. The method permitted to detect most of the steroid esters in equine and bovine blood. The proposed procedure was successfully applied to the analysis of equine and bovine blood samples. The 
main steroids found in the four samples was cortisol, followed by corticosterone, androstenedione and dexamethasone isonicotinate. Androsterone and DHEA were not detected in any sample. In conclusion, the present method allows identification and quantification of steroids and performance are increasing hormones, and it could be used when fraudulent use is suspected in racing animals, in an equine trade or to control the healthy state of these animals. 


\subsection{Simultaneous quantitation of 9 anabolic and natural steroidal hormones in equine urine by UHPLC-MSIMS.}

This work aimed to set up a new UHPLC-MS/MS triple quadrupole method able to detect and quantify nine anabolic steroids in horse Urine. Quantifiable steroids in the proposed method are as follows: Deslorelin (DES), Dexamethasone Sodium Phosphate (Desa NaP), Prednisolone (PRE), Methylprednisolone (Me-PRE), Stanozolol (STA), Boldenone (BOL), Nandrolone (NAN), Dexamethasone Isonicotinate (Desa ICo) and Altrenogest (ALT). Most of the anabolic substances included in this sub-chapter are commonly used as doping in horse racing. The proposed procedure has easy sample preparation; it is fast, sensitive, accurate and robust.

\subsubsection{Results and discussion}

\section{Method validation}

The method was fully validated in terms of linearity, limit of detection (LOD), LOQs, accuracy, precision and recovery.

\section{Precision and Linearity}

Precision was calculated intra and inter-day with LOQ, CM, CU, and U1 from a three-day validation of five daily repetitions (Table 14). The outcome is expressed according to the coefficient of variation (CV\%). The CV is within the range $0.98-16.54 \%$.

To calculate the linearity of the proposed method, two calibration curves were created using all concentrations between LOQ and CU (low-range standard curve, 5 points, 5-day validation) and all the concentrations between the LOQ and U4 (highrange standard curve, 9 points, 5-day validation). Obtained linearity expressed as the coefficient of linear regression $\left(R^{2}\right)$ is higher than $0.9955 \%$ (Table 14).

LODs and LOQs for studied compounds are within a range of 0.1-0.5 and 0.25$5 \mu \mathrm{g} / \mathrm{l}$, respectively. These values are similar to or lower than the limits described in the literature. 


\begin{tabular}{|c|c|c|c|c|c|c|c|c|c|}
\hline \multirow{3}{*}{ 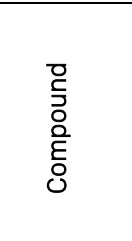 } & \multicolumn{8}{|c|}{ Precision $(\mathrm{CV}, \%)$} & \multirow{3}{*}{$\begin{array}{c}\text { Linearity } \\
\text { (R2) }\end{array}$} \\
\hline & \multicolumn{2}{|c|}{$\begin{array}{c}\text { Low } \\
\text { concentration }\end{array}$} & \multicolumn{2}{|c|}{$\begin{array}{c}\text { Medium } \\
\text { concentration }\end{array}$} & \multicolumn{2}{|c|}{$\mathrm{CU}$} & \multicolumn{2}{|c|}{ U1 } & \\
\hline & $\begin{array}{l}\text { Intra- } \\
\text { day }\end{array}$ & $\begin{array}{l}\text { Inter- } \\
\text { day }\end{array}$ & $\begin{array}{l}\text { Intra- } \\
\text { day }\end{array}$ & $\begin{array}{l}\text { Inter- } \\
\text { day }\end{array}$ & $\begin{array}{l}\text { Intra- } \\
\text { day }\end{array}$ & $\begin{array}{l}\text { Inter- } \\
\text { day }\end{array}$ & $\begin{array}{l}\text { Intra- } \\
\text { day }\end{array}$ & $\begin{array}{l}\text { Inter- } \\
\text { day }\end{array}$ & \\
\hline des & 11.52 & 12.22 & 6.30 & 7.01 & 2.25 & 2.80 & 0.98 & 1.15 & 0.9997 \\
\hline Desa NaP & 15.64 & 16.54 & 10.54 & 11.91 & 5.56 & 6.65 & 2.01 & 2.85 & 0.9955 \\
\hline pred & 10.84 & 11.80 & 7.54 & 8.25 & 4.41 & 5.01 & 2.24 & 2.59 & 0.9965 \\
\hline Me-pre & 14.32 & 14.98 & 8.54 & 9.12 & 2.39 & 3.35 & 2.57 & 3.35 & 0.9974 \\
\hline sta & 13.33 & 14.54 & 8.25 & 9.68 & 3.57 & 5.87 & 3.01 & 3.87 & 0.9968 \\
\hline bol & 12.15 & 13.13 & 7.70 & 8.80 & 2.32 & 3.98 & 2.14 & 2.54 & 0.9988 \\
\hline nan & 13.54 & 15.19 & 7.87 & 8.57 & 2.54 & 3.58 & 2.06 & 2.25 & 0.9986 \\
\hline Desa Iso & 10.15 & 12.12 & 6.54 & 7.99 & 2.14 & 3.47 & 1.54 & 1.99 & 0.9975 \\
\hline alt & 12.69 & 12.99 & 7.69 & 9.02 & 3.57 & 5.87 & 1.87 & 2.00 & 0.9987 \\
\hline
\end{tabular}

\section{Accuracy and recovery}

The accuracy (intra-day and inter-day, three-day validation) was calculated from C1, C2, and U3 concentrations. The results are displayed in Table 15 and expressed in terms of 'relative error percent' (RE\%). The RE\% is within the range $0.84-13.98 \%$ for all compounds.

Recovery was studied by spiking normal equine urine with a mixture standard of the nine hormones. The recovery value was obtained using the following formula: $\left(\left(A_{\text {se }}-A_{\text {blank }}\right) / A_{\text {std }}\right) \times 100$, where $A_{\text {se }}$ is the area about the urine enriched with a low concentration (C1 and CM) of all compounds, $A_{\text {blank }}$ is the area of analytes detected in urine and $A_{\text {std }}$ is the area of a mixture standard of all the compounds dissolved in methanol. Recoveries obtained by spiking the matrix at the CM concentration were in the range of $94.16-98.55 \%$, with a CV lower than $1.69 \%$ (Table 15). 


\begin{tabular}{|c|c|c|c|c|c|c|c|c|c|c|}
\hline \multirow{3}{*}{ 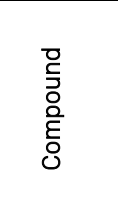 } & \multicolumn{6}{|c|}{ Accuracy } & \multicolumn{4}{|c|}{ Recovery } \\
\hline & \multicolumn{2}{|c|}{$\mathrm{C} 1$} & \multicolumn{2}{|c|}{$\mathrm{C} 2$} & \multicolumn{2}{|c|}{ U1 } & \multicolumn{2}{|l|}{$\mathrm{CM}$} & \multicolumn{2}{|l|}{$\mathrm{C} 1$} \\
\hline & $\begin{array}{c}\text { Intra- } \\
\text { day } \\
(\mathrm{RE}, \%)\end{array}$ & $\begin{array}{l}\text { Inter- } \\
\text { day } \\
(\mathrm{RE}, \%)\end{array}$ & $\begin{array}{l}\text { Intra- } \\
\text { day } \\
\left(\mathrm{RE}_{1} \%\right)\end{array}$ & $\begin{array}{l}\text { Inter- } \\
\text { day } \\
(\mathrm{RE}, \%)\end{array}$ & $\begin{array}{l}\text { Intra- } \\
\text { day } \\
(\mathrm{RE}, \%)\end{array}$ & $\begin{array}{l}\text { Inter- } \\
\text { day } \\
(\mathrm{RE}, \%)\end{array}$ & $\begin{array}{c}\text { Recovery } \mathrm{n} \\
=3\end{array}$ & $\begin{array}{l}C V, \\
\%\end{array}$ & $\begin{array}{l}\text { Recovery } n \\
=3\end{array}$ & $\begin{array}{c}\mathrm{CV}, \\
\%\end{array}$ \\
\hline des & 7.54 & 7.78 & 2.25 & 2.58 & 1.98 & 2.36 & 98.36 & 1.25 & 95.75 & 3.15 \\
\hline Desa NaP & 13.30 & 13.98 & 6.65 & 6.97 & 2.36 & 2.86 & 94.16 & 1.69 & 89.12 & 6.02 \\
\hline pred & 8.54 & 8.75 & 1.98 & 2.54 & 0.99 & 1.22 & 96.54 & 0.99 & 92.15 & 5.15 \\
\hline Me-pre & 7.54 & 7.66 & 1.68 & 2.15 & 0.84 & 1.14 & 97.25 & 0.94 & 94.12 & 4.87 \\
\hline sta & 9.36 & 9.68 & 3.47 & 4.15 & 1.98 & 2.00 & 95.37 & 1.14 & 89.99 & 3.99 \\
\hline bol & 8.85 & 8.86 & 2.48 & 3.30 & 1.15 & 1.87 & 96.12 & 1.01 & 91.25 & 4.15 \\
\hline nan & 7.99 & 8.25 & 3.12 & 3.96 & 1.58 & 1.88 & 98.55 & 1.09 & 92.75 & 3.02 \\
\hline Desa Iso & 10.15 & 11.12 & 5.69 & 5.99 & 2.58 & 2.69 & 97.12 & 0.94 & 93.16 & 3.12 \\
\hline alt & 7.09 & 8.01 & 3.68 & 4.41 & 2.01 & 2.20 & 97.00 & 0.75 & 94.84 & 3.68 \\
\hline
\end{tabular}

\section{Specificity}

As for the paper from Genangeli et al., high specificity was calculated by monitoring the retention time of parent/daughter ions for all the analytes over time (five-day period, $n=25)$. The retention times for this method were stable, with RSD percent values $\leq 0.98 \%$.

Specific precursor/product ion transitions were identified for each steroid, and the multiple reaction monitoring transitions with the most abundant product ion were selected for quantitation, and other product ions were selected for qualification (Table 4).

\subsubsection{Conclusions}

A new UHPLC-MS/MS method for the simultaneous quantitation of 9 anabolic and natural steroidal hormones in equine urine was developed. This procedure allows the detection of 9 anabolic steroids in equine urine samples. The procedure is not time-consuming, intuitive and with easy sample preparation. The reported method shows excellent performances regarding specificity, sensitivity (LOQ in the range 
0.25-5.0 $\mu \mathrm{g} / \mathrm{l})$ and linearity. The proposed analytical procedure allows to easily and rapidly quantify a large number of anabolic compounds in less than ten minutes. The compounds included in this method are the most common anabolic steroids abused in horse racings. In conclusion, the reported procedure allows to simultaneously identify and quantify nine anabolic steroids in a short period. The proposed method could be applied for the control of racing and sports-related animals.

\subsection{Chapter conclusions}

The present chapter reported three applications of High-Performance Liquid Chromatography (HPLC) Triple Quadrupole Mass Spectrometry (QQQMS) for an easy quantification and qualification of endogenous and exogenous steroids in liquid biological samples. Precisely, the steroids were qualified and quantified in serum, blood and urine.

The high accuracy, precision, sensitivity and overall performances of the proposed methods led to the development of new versatile methods with an easy and fast sample preparation. Additionally, when compared to other analytical methodologies, the high specificity provided by the triple quadrupole allows to quantify compounds in complex matrices without false positive or false negative results. 



\section{Chapter 3}

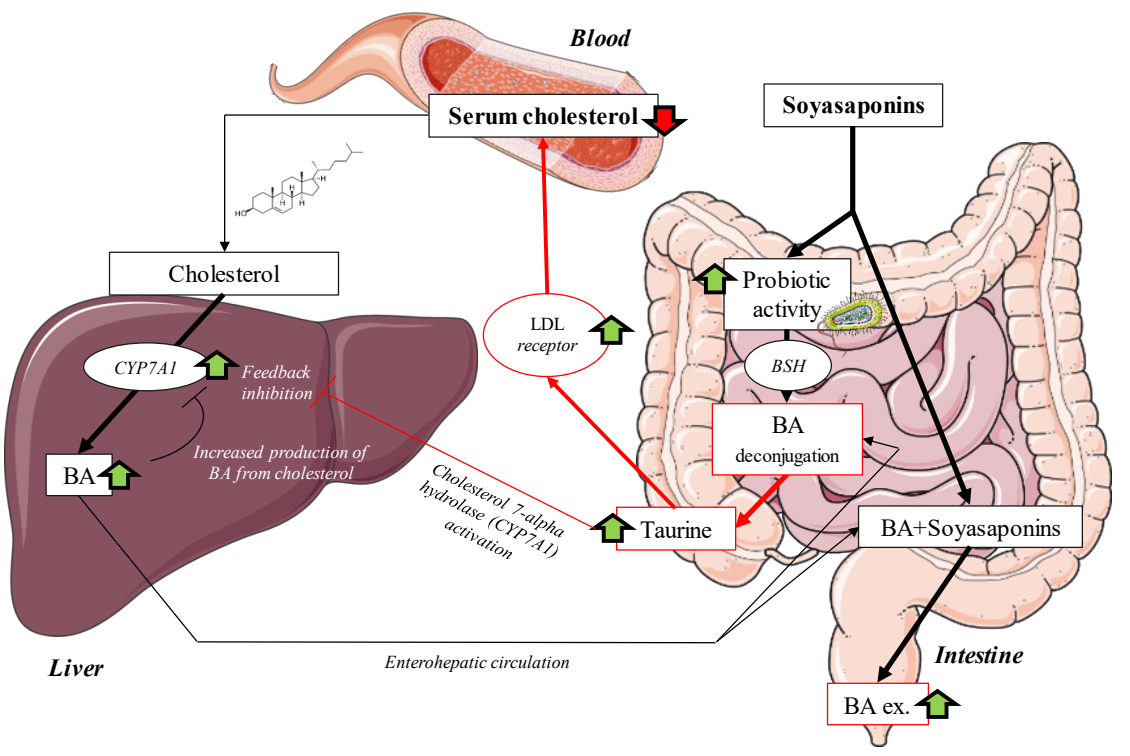

Adapted from:

MALDI-mass spectrometry imaging to investigate lipid and bile acid modifications caused by lentil extract used as a potential hypocholesterolemic treatment

Michele Genangeli, Annemarie Heijens, Alice Rustichelli, Noortje Dien Schuit, Maria Vittoria Micioni Di Bonaventura, Carlo Cifani, Sauro Vittori, Tiffany Porta Siegel and Ron M.A. Heeren

Journal of the American Society for Mass Spectrometry 2019, DOl: 10.1007/s13361-019-02265-9 


\section{Mass spectrometry imaging for a better understanding of biological pathways}

\subsection{Abstract}

This chapter reports matrix-assisted laser desorption/ionization mass spectrometry imaging to investigate systematic effects of a lentil extract treatment to lower cholesterol and expand prior findings involving LC-MS applications. For this purpose, mass spectrometry imaging was used to spatially investigate modifications in the lipid composition and cholesterol levels in brain, liver and intestines as well as bile acids in the liver and intestine of rats treated with lentil extract. Neither the lipid composition nor cholesterol levels in the brain samples were found to be significantly different between the treated and not treated animal groups. The hypercholesterolemic livers showed signs of steatosis (lipid marker PG 36:4), but no modifications in bile acid, cholesterol and lipid composition. We found significant differences ( $U \cup C$ > $0.75)$ in the intestines regarding bile acid and lipid composition after treatment with the lentil extract. Treated rats showed a decreased reabsorption (increased excretion) of ursodeoxycholic acid, deoxycholic acid, chenodeoxycholic acid and an increased deconjugation of taurine-conjugated bile acids (taurochenodeoxycholic acid, taurodeoxycholic acid, taurocholic acid and 3-keto-taurocholic acid). This indicates that the lentil extract lowers the total cholesterol level in two synergic ways: i) it increases the excretion of bile acids, hence new bile acids are produced in the liver from serum cholesterol; ii) the prebiotic effect leads to free taurine which upregulates the de-novo synthesis of bile acid from cholesterol while activating LDL receptors. We demonstrate here that mass spectrometry imaging is a valuable tool for a better understanding of the effects of treatments such as for the synergistic cholesterol-lowering effect of the lentil extract. 


\subsection{Introduction}

Nutrition has been known to affect a plethora of organs and functions in the body, making it an important factor in health research[91, 92]. This study aims to add to this knowledge by investigating the influence of nutrition and local cellular metabolism in the liver, intestines, and brain to provide insight into the effects of consuming vegetable extracts and phytocomplexes on the body. We focus here on lentils, which is a type of legume high in macronutrients, mostly proteins and carbohydrates, but also rich in bioactive compounds. Regular consumption of lentils has been shown to decrease the risk for various diseases such as diabetes, obesity, cancers and cardiovascular diseases due to the presence of bioactive compounds (e.g. polyphenols known to have health-promoting effects) [93]. The carbohydrates in lentils act as prebiotic components and are beneficial for the growth of healthy microbiota [94]. A recent publication from Micioni Di Bonaventura et al. investigating the effect of lentil extract (LE) on the cholesterol levels in rats via high performance liquid chromatography-tandem mass spectrometry (HPLC-MS/MS) concluded that a LE (Lens culinaris Medik) treatment lowered the total plasma cholesterol and LDL levels due to an increase in the excretion of fecal bile acids $[45,95]$. This cholesterollowering effect was a result of the inhibition of intestinal reabsorption of bile acids caused by an active phytochemical in lentils called soyasaponin $[45,96,97]$. There are two groups of soyasaponins known as groups A and B; group B contains compounds, e.g., soyasaponin I and $\beta$ g, which are mainly responsible for lowering cholesterol [45, 98].

Bile acids are produced by hepatocytes from precursor cholesterol and secreted via the bile duct into the duodenum [99]. [100]. Most bile acids (around 95\%) are reabsorbed in the ileum and transported back to the liver via the portal vein (the enterohepatic circulatory system) [99]. Primary bile acids are produced in the hepatocytes of the liver and then converted into secondary bile acids by the intestine microbiota. The primary bile acids, mainly cholic acid (CA) and chenodeoxycholic acid (CDCA), are conjugated with taurine and glycine to be excreted as bile into the duodenum [101]. In the intestines, the microbiota converts CA into deoxycholic acid 
(DCA) and CDCA into lithocholic acid (LCA) and ursodeoxycholic acid (UDCA), which are secondary bile acids [101, 102]. Because soyasaponins have an inhibiting effect on the reabsorption of bile acids, it is of great interest to investigate the metabolic modifications of bile acid and lipid compositions in the intestines after treatment with soyasaponin [45].

It was hypothesized that the changes in the intestine after LE treatment [45] could also result from altered bile acid synthesis and modifications in the lipidic composition in the liver. Additionally, we speculated that the gut-brain connection and the hypercholesterolemia altered the expression of lipids and cholesterol levels in the brain. Here, we employed matrix-assisted laser desorption/ionization mass spectrometry imaging (MALDI-MSI) to analyze organs harvested from the same animals sacrificed in the paper from Micioni Di Bonaventura et al. (Figure 13), and investigate whether LE food supplementation affects cholesterol and lipid levels of the brain, intestines, and liver, and bile acid levels in the intestines and liver [103-107]. MALDI-MSI has been proven to be an extremely valuable tool able to study the spatial distribution of a wide variety of analytes (e.g., lipid, protein, peptide, metabolites) with high sensitivity [108-110]. Compared to conventional imaging techniques such as PET/CT or MRI, MALDI-MSI offers numerous advantages: it is a labelfree technique that can produce higher spatial resolution images and allows the simultaneous detection and identification of thousands of different compounds in a single experiment [111]. Additionally, when compared to other mass spectrometry techniques such as HPLC-MS/MS, MALDI-MS allows the analysis and visualization of selected molecules while maintaining their spatial distribution and the integrity of the sample.

We discovered that LE has a duplex effect in which it lowers the total cholesterol and LDL levels, where $\mathrm{N}$-arachinodoil Taurine and Taurine conjugated BA play an important role in these effects. Furthermore, our findings show that LE does not interfere with brain and liver functionality. 


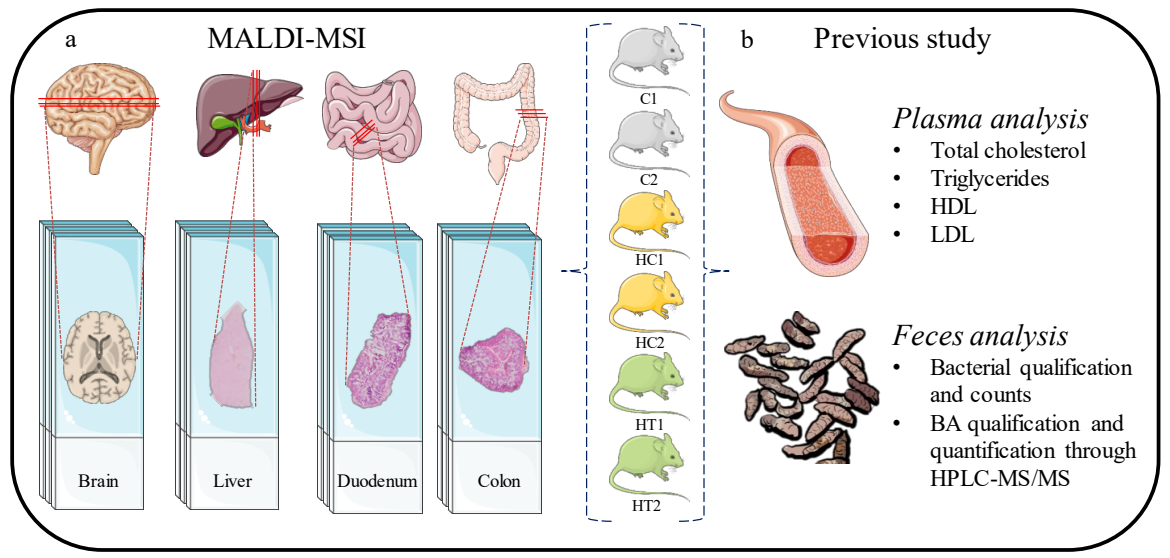

Figure 13 - [a] Experimental design. A total of six rats (2x control [C], 2x hypercholesterolemic control [HC] and $2 x$ hypercholesterolemic treated [HT]) were sacrificed. Four organs were collected from each rat (brain, liver, duodenum and colon), sectioned and analyzed in triplicate. [b] The animals included in this paper are the same rats used for the paper from Micioni Di Bonaventura et al., where the listed analysis were performed [45].

\subsection{Materials and methods}

\subsubsection{Chemicals}

Methanol, ethanol, n-Hexane and xylene (ULC/MS-CC/SFC grade) were purchased from Biosolve Chimie (Dieuze, France). Red phosphorous standard, acetone, entellan and norhamane were purchased from Merck KGaA (Darmstadt, Germany).

\subsubsection{Animals and organ collection}

The organs used for this study were harvested from the same animals sacrificed as described in the paper from Micioni Di Bonaventura et al. [45] (Figure 13). The brains, livers, duodena and colons from 6 male Sprague-Dawley rats $(n=24)$ were analyzed in three technical replicates. The animals belonged to three study groups as follow:

i) two rats were used as a control group and did not receive any treatment (C1 and C2) but were fed with standard laboratory chow and water ad libitum (4RF18, Mucedola, Settimo Milanese, Italy; $2.6 \mathrm{kcal} / \mathrm{g}$ ); 
ii) two rats received a commercially available high cholesterol diet (AIN76A rodent diet with 1\% cholesterol and 0.5\% cholic acid, D04082702 Research Diet, New Brunswick, USA) to cause hypercholesterolemia and also received water ad libitum (hypercholesterolemia rats: HC1 and HC2);

iii) The remaining two rats were fed with the same high cholesterol diet and also received $4 \mathrm{ml}$ of LE (Lens culinaris Medik) rich in soyasaponin diluted in $16 \mathrm{ml}$ of water, as described elsewhere [3]. When they finished drinking the extract, free access to water was allowed (hypercholesterolemia rats with treatment: HT1 and HT2).

All rats were sacrificed after 71 days, and organs harvested. The intestines were flushed with water. The organs were stored separately in tin foil in a plastic bag and kept in a $-80^{\circ} \mathrm{C}$ freezer prior to analysis.

\subsubsection{Preparation of tissue sections for the MALDI-MSI analysis}

The organs were sectioned with a cryo-microtome (Leica CM 1860 UV) at $12-\mu m$ thickness in a 30-45\% humidity atmosphere. The brain was transversally sectioned, the median lobe of the liver was axially sectioned, and consecutive transverse cross-sections of the intestines (after the duodenalpapillae for the duodenum) were also prepared. The tissue sections were mounted onto clean indium tin oxide (ITO) unpolished float glass slides (25 x $75 \times 1.1 \mathrm{~mm}, \mathrm{RS}=4-8 \Omega$, Delta Technologies LTD, Loveland, USA). The ITO glass slides were cleaned by sonicating for 6 min in subsequent baths of $n$-hexane and ethanol. Dust particles from the slides were removed using nitrogen before usage. The organs were randomly mounted onto the slides in order to minimize batch effects during analysis. Prior to analysis, the mounted tissue sections were desiccated under vacuum for 20 min. Then, 80-90 mg of norharmane MALDI matrix was dissolved in $2 \mathrm{ml}$ of methanol. This solution was applied onto the slides with a sublimator device (HTX Technologies, LLC, Chapel Hill, USA) [112], and samples were allowed to dry in in the vacuum desiccator for at least 
20 min. Each plate was scanned (grayscale, 2400 dpi) to align the laser with a visual image. The plates were stored in vacuum bags in the $-80{ }^{\circ} \mathrm{C}$ freezer prior to MALDITOF analysis.

\subsubsection{MALDI-MSI analysis}

Lipid mass spectra were acquired with the Bruker RapifleX MALDI Tissuetyper (Bruker Daltonik GmbH, Bremen, Germany) where ions were generated with a Smartbeam 3D laser in M5 profile ( $N d: Y A G$ laser, $\lambda=355 \mathrm{~nm}$ ) and data acquired at a spatial resolution of $50 \mu \mathrm{m}$. Experiments were performed in negative ionization mode over a mass range of m/z 300-1700. . The laser intensity was set between 70-90\%. External mass calibration was performed using a red phosphorus standard mixed with acetone [113]. MS/MS analysis was performed on a Synapt G2-Si (Waters, Manchester, UK) which utilizes collision-induced dissociation (CID) to fragment isolated precursor ions in the trap cell with a collision energy of $25 \mathrm{eV}$ and an isolation window of $1 \mathrm{Da}$. Exact mass measurements were performed on a Fourier-transform ion cyclotron resonance (FT-ICR) MS system (solarix; Bruker Daltonik GmbH, Bremen, Germany), equipped with a 9.4-T magnet to provide further information for the elucidation of the identity of selected ion species. This is deployed to match the observed $m / z$ values with the theoretical mass of the molecule under study and complements the MALDITOF-based MS/MS identification.

\subsubsection{Data pre-processing and analysis}

The resulting data from the analysis of all organs were normalized to total ion count (TIC) using FlexImaging (version 4.1, Bruker Daltonik GmbH, Bremen, Germany). Average mass spectra from each organ were exported to mMass (http://www.mmass.org, version 5.5.0) where a Gaussian baseline correction was applied. A peak list was generated with a binning of 0.2 bandwidth and used for further data analysis. Multivariate analysis (i.e., unsupervised principal component analysis (PCA) and probabilistic latent semantic analysis (PLSA) were performed on the TICnormalized spectra using SCiLS Lab software (version 2016b, Bremen, Germany). The 
PCA was used to find the highest variance and check the quality of the data, while the pLSA was employed to discover differences between groups and identify potential features responsible for these differences. For each PCA and pLSA analysis, a total of 15 components was taken, and the scores plots were studied to find components and $m / z$ values which discriminate between groups $\mathrm{C}, \mathrm{HC}$ and $\mathrm{HT}$.

First, the brain samples were analyzed by comparing the whole tissue section at first, and then by comparing then selected regions of interest of the brains (i.e., prelimbic cortex, caudate putamen, cerebellum, and hippocampus (Figure 19a). These areas were chosen as previous studies showed that hypercholesterolemia could induce changes in the hippocampal phenotype and memory deficits $[114,115]$. The liver samples were analyzed by comparing the whole tissue section and, for the intestines, the duodenum and the colon were analyzed independently.

A list containing peaks of interest related to bile acids, cholesterol and steatotic liver-associated disease was created based on literature (Table 16). The peaks belonging to the aforementioned peak list were visually and statistically examined. The most relevant components from the PCA and LDA were further analyzed by evaluating the receiver operating characteristic (ROC) plot of every discriminative (AUC $\geq 0,75)$ peak obtained. Selected peaks with differences between groups or sub-groups were then analyzed on a Synapt G2Si using MS/MS in order to obtain additional information to identify these peaks, and confirmed using the exact mass from the FT-ICR.

Table 16 - Peak list generated from the literature containing expected peaks with relative $\mathrm{m} / \mathrm{z}$ values in negative ion mode $[5,17]$.

\begin{tabular}{cccc}
\hline Molecule & $\mathrm{m} / \mathrm{z}[\mathrm{M}-\mathrm{H}]-$ & Molecule & $\mathrm{m} / \mathrm{z}[\mathrm{M}-\mathrm{H}]-$ \\
\hline Lithocholic acid (LC) (S) & 375.57 & PA (18:2_18:2) & 695.47 \\
Cholesterol & 385.65 & PA (18:1_18:2) & 697.48
\end{tabular}




\begin{tabular}{|c|c|c|c|}
\hline Deoxycholic acid (DC) (S) & 391.58 & PE (16:0_18:2) & 714.51 \\
\hline $\begin{array}{l}\text { Chenodeoxycholic acid } \\
\text { (CDC) (P) }\end{array}$ & 391.58 & PE (18:0_18:2) & 742.54 \\
\hline $\begin{array}{l}\text { Ursodeoxycholic acid (UDC) } \\
\text { (S) }\end{array}$ & 391.58 & PE (18:0_20:4) & 766.54 \\
\hline $\begin{array}{l}\text { Hyodeoxycholic acid (HDC) } \\
\text { (S) }\end{array}$ & 391.58 & PE (18:0_22:4) & 794.57 \\
\hline Cholic acid (CA) & 407.57 & PI (16:9_18:2) & 833.52 \\
\hline $\begin{array}{l}\text { Glycochenodeoxycholic acid } \\
\text { (GCDC) (C) }\end{array}$ & 448.62 & PI (16:0_20:4) & 857.52 \\
\hline Glycocholic acid (GC) (C) & 464.63 & PI (18:0_18:2) & 861.55 \\
\hline $\begin{array}{l}\text { Taurochenodeoxycholic acid } \\
\text { (TCDC) (C) }\end{array}$ & 498.71 & PI (18:0_20:4) & 885.55 \\
\hline Taurocholic acid (TC) (C) & 514.71 & Cardiolipin & 1465.00 \\
\hline
\end{tabular}

\subsubsection{Histological staining}

The matrix was removed from the tissue sections after MALDI-MSI analysis in a bath of $100 \%$ ethanol (2x) for 3 min and consecutively rehydrated in subsequent ethanol baths of $96 \%(2 \times)$ and $70 \%$ (2x), each for 2 min. The samples were stained with hematoxylin for 3 min, washed with deionized water and stained with eosin for 30 sec. The slides were subsequently washed under running tap-water and placed in absolute ethanol for 1 min and fresh xylene for 30 sec. Finally, the slides were coverslipped and fixed with entellan. High-resolution optical images of the H\&E-stained tissue microarrays were obtained with the MIRAX Desk Scanner (Zeiss, Jena, Germany).

\subsection{Results and discussion}

\subsubsection{Cholesterol, bile acid and lipid modifications in the liver}

In the previous study involving the same rats, LE was found to reduce the levels of cholesterol effect in plasma. Here, we focused our investigations on the levels of cholesterol in the liver. The control group was expected to contain the lowest cholesterol levels, followed by the hypercholesterolemic treated group, and the hypercholesterolemic control group which displayed the highest levels. In the present 
study however, no significant differences in cholesterol levels in the liver were found among the three groups (Figure 14a).

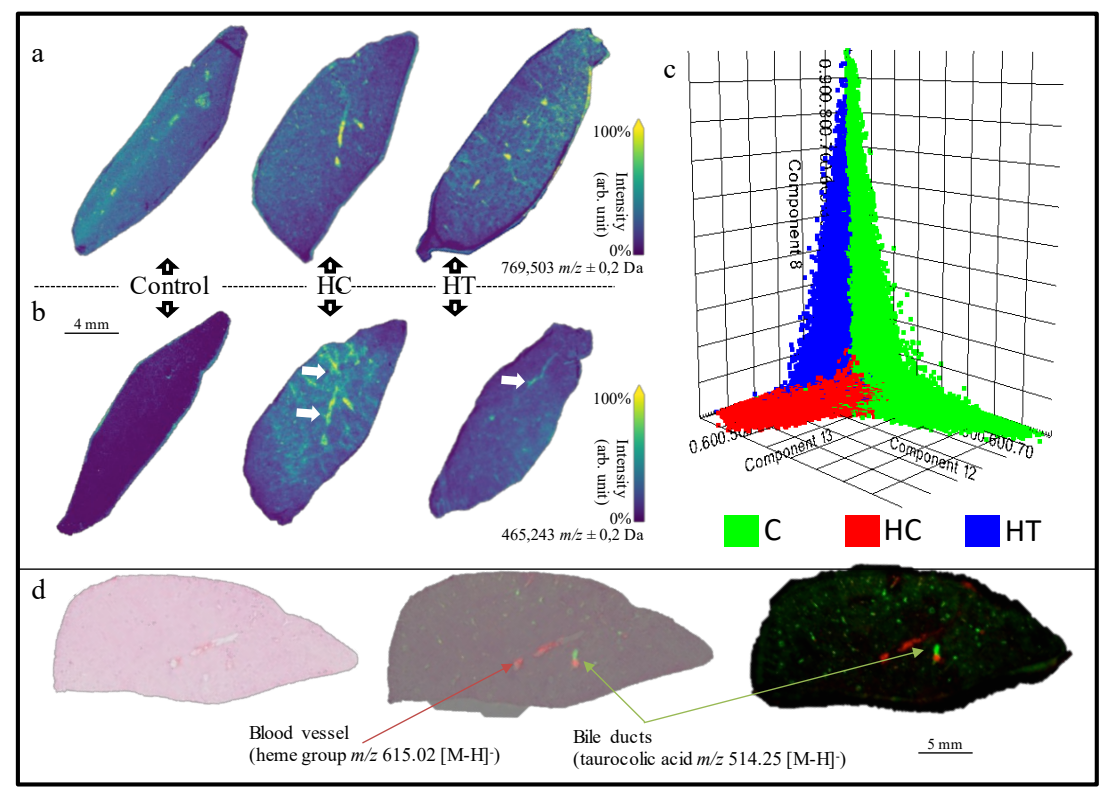

Figure 14 - [a] Molecular distribution of the cholesterol sulfate. From the image, there are no differences regarding cholesterol expression in the three liver groups. [b] Molecular image of the mass peak corresponding to PG 36:4 (a marker for steatosis, as indicated by the white arrows). Results show that the signal measured for PG is relatively higher in the HC liver compared to the treated group and control. [c] Unsupervised statistical analysis (pLSA) showing a clear distribution of the three groups (Green = control, Red = Hypercholesterolemic control and Blue = Hypercholesterolemic treated). [d] Blood vessels and bile ducts highlighted using the heme group (m/z $\left.615.02[\mathrm{M}-\mathrm{HI}]^{-}\right)$and the taurocholic acid $(\mathrm{m} / \mathrm{z} 514.25[\mathrm{M}-\mathrm{H}] \mathrm{]})$, respectively.

Next, we investigated the bile acids (BA) - produced from precursor cholesterol and secreted via the bile duct and lipids levels. The bile ducts were identified by localizing and visualizing taurocholic acid ([M-H]- at $m / z$ 514.25) (Figure 14d). The peaks included in the generated peak list (Table 16) and related to BA and steatotic liver-associated disease, were visually and statistically examined. The $m / z$ values were then compared with the significant peaks from the pLSA. Molecular images of selected BA (Table 16) [45] did not show differences in the distribution of these molecules between the three groups.

With respect to lipids, pLSA analysis of the whole liver sections displayed three components distinguished the three different groups of treated mice 
(Figure 14c). From the loading plot, a total of 218 peaks contributed to this discrimination, of which 11- related to lipid species - were found to be statistically relevant (AUC > 0.75) for the discrimination between two or all three groups. These peaks were identified with MS/MS and confirmed using the exact mass from the FT-ICR (Table A 2, Figure 15). PE (18:0_20:4), PE (18:0_22:4), PI (16:0_20:4) and PI (18:0_20:4) were mainly expressed in the control group in comparison to the other two groups and they mostly contain a chain of arachidonic acid. PI with a fatty acid chain of arachidonic acid (20:4) has been shown to be connected with a healthy liver, in line with our observations. PE (16:0_18:2), PI (16:0_18:2), and PI (18:0_18:2) did not show noticeable differences between groups, but only differences between individual organs.

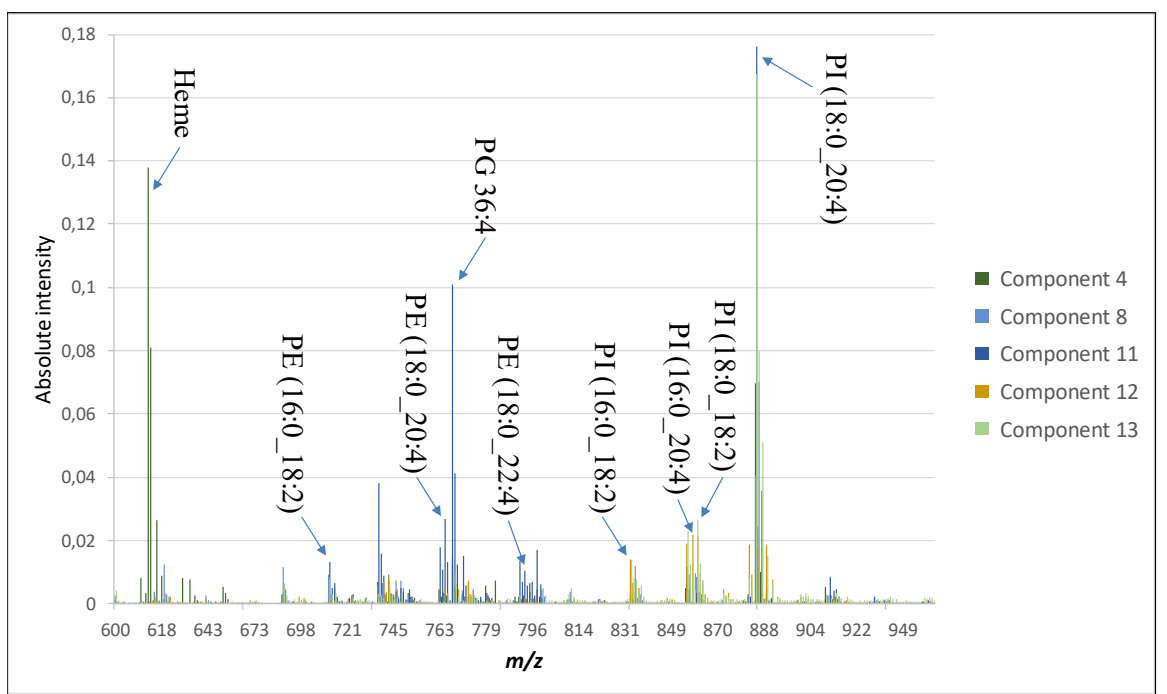

Figure 15 - Loading plot from the major components of the PLSA in the range $\mathrm{m} / \mathrm{z} 600-1050$ for the liver groups (C, HC, HT). The identified lipids described in this paper are highlighted. Component 4 is mainly representative for the Heme group. Component 11 is mainly representative for PE(16:0_18:2), PE(18:0_20:4), PG36:4, PE(18:0_22:4). Component 12 and 13 are mainly representative from all the $\mathrm{Pl}$.

The last four identified lipids were: PG (36:4), known to be associated with steatotic livers, and PE (40:6), PG (38:5), PI (16:0_22:6). These four lipids displayed higher levels in the HC group (Figure 14b).

Nor the cholesterol and BA expression in the liver were statistically relevant for discrimination between groups. On the other hand, the $\mathrm{C}$ groups showed a higher expression in PI with a fatty acid chain of arachidonic acid (20:4), fatty acid associated 
with healthy liver [105]. However, we showed that the HC group showed higher signals of a steatosis-associated lipid.

Table 17 - List of bile acids, soyasaponins and N-Arachidonoyl Taurine, confirmed with the exact mass. Starting from the left column we have the compound name, theoretical mass, measured mass and error expressed in ppom from a low mass resolution (Rapiflex) and a high mass resolution (Solarix) instruments. Soyasaponins were not found in the analyzed tissues.

\begin{tabular}{llllll}
\hline & & Rapiflex & Rapiflex & Solarix & Solarix \\
\cline { 4 - 6 } Compounds $[\mathrm{M}-\mathrm{H}+]$ & Theor. mass & measured $\mathrm{m} / \mathbf{z}$ & ppm err. & $\begin{array}{l}\text { measured } \\
\mathrm{m} / \mathbf{z}\end{array}$ & ppm err. \\
\hline CA & 407,28029 & 407,3 & -48 & 407,2805 & $-0,5$ \\
LCA & 375,29046 & 375,3 & -25 & 375,2909 & $-1,1$ \\
DCA & 391,28538 & 391,2 & 218 & 391,2850 & 0,9 \\
CDCA & 391,28538 & 391,2 & 218 & 391,2850 & 0,9 \\
UDCA & 391,28538 & 391,2 & 218 & 391,2850 & 0,9 \\
TDCA & 498,28948 & 498,3 & -21 & 498,2904 & $-1,8$ \\
TCDCA & 498,28948 & 498,3 & -21 & 498,2904 & $-1,8$ \\
3keto-TCA & 512,26874 & 512,3 & -61 & 512,2689 & $-0,3$ \\
TCA & 514,28439 & 514,3 & -30 & 514,2837 & 1,3 \\
N-Arachidonoyl Taurine & 410,23705 & 410,2 & 90 & 410,2375 & $-1,0$ \\
SOYA & 941,51153 & NA & NA & NA & NA \\
SOYA BG & 1067,54323 & NA & NA & NA & NA \\
\hline & & & & & \\
\hline
\end{tabular}

\subsubsection{Cholesterol, lipid and bile acid modifications in the intestine (colon and duodenum)}

Next, we examined the differences in cholesterol levels in the intestines.

In negative ionization mode, cholesterol can be detected as cholesterol sulfate, which is typically detected at $m / z 465$ and $m / z 467$ [116]. Both of these peaks were present in the obtained spectra, but they were not discriminating between 
the groups. Their distribution was similar for both the colon and the duodenum (Figure 16a)

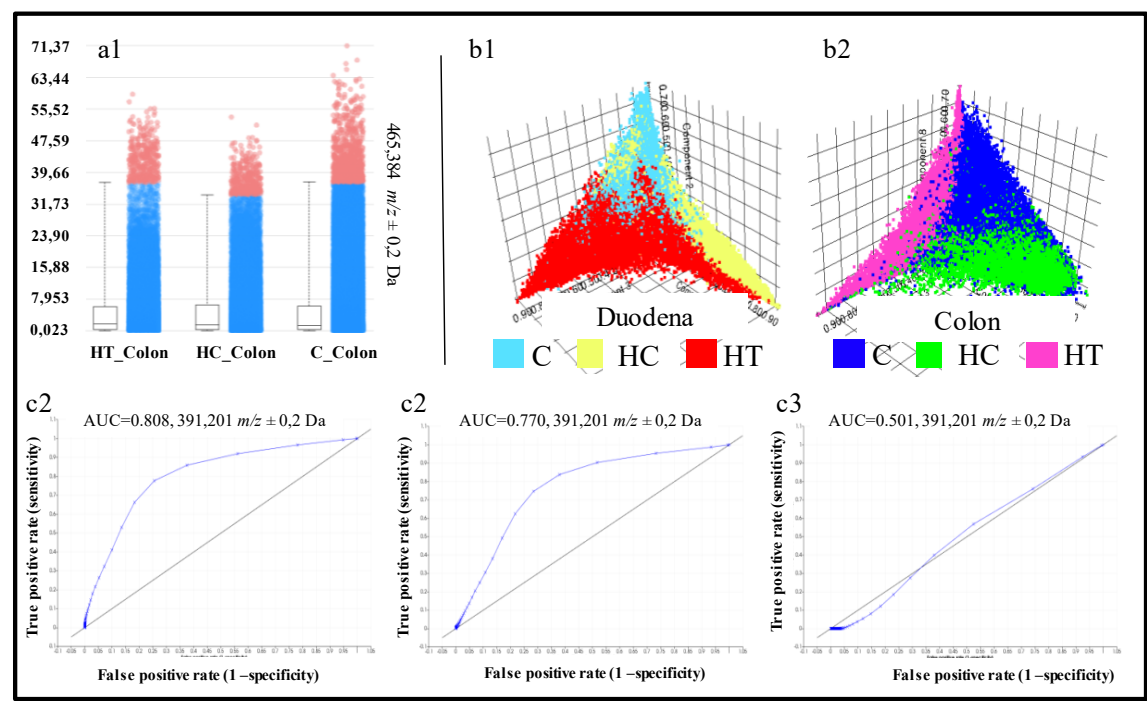

Figure 16 - [a] Cholesterol level in the three colon groups. No significant variations were showed. [b1/2] Scores plot from the pLSA analysis between the three duodena groups (b1, C= light blue, HC=yellow, HT=red) and between the three colon groups (b2, $C=$ blue, $H C=$ green, $H T=p i n k)$. Both the $\mathrm{pLSA}$ show differences between the groups, especially in the colon. [c1-3] ROC plots for peak m/z 391.2 (CDCA/DCA/UDCA) which show significant differences in the expression of these BA in the (C1) HT_Colon vs C_Colon, (C2) HT_Colon vs HC Colon and no differences in the (c3) HT_Duodenum vs HC_Duodenum.

With respect to the lipid composition in the intestines, no significant differences were observed between groups (data not shown). A possible reason for this is that the LE treatment rich in soyasaponins only has an effect on the bile acid composition via the microbiota, and might not directly affect the lipid composition. We investigated the presence of soyasaponin I and $\beta g$, the cholesterol-lowering agents found in lentils, with $m / z 941.5$ and $m / z$ 1067.0, respectively [117]. Additionally, peaks related to the conjugation of soyasaponins and bile acids were also examined. However, no peaks were found, indicating that no soyasaponins could be detected in the intestines.

Even though we did not find differences in the cholesterol and lipid distribution in the intestine and did not detect any soyasaponins, the PCA showed different variance within both the duodenum $(n=3)$ and colon $(n=3)$ groups, analyzed independently (Figure 16b). The HT and HC groups showed a clear separation, with both overlapping the control group (Figure 16b). We identified a total of $58 \mathrm{~m} / z$ values 
able to discriminate between $\mathrm{HT}$ and $\mathrm{HC}$ groups both in the duodenum and colon with an $A \cup C \geq 0.75$. Comparing these 58 peaks with the list of bile acids quantified from Bonaventura et al. , we identified chenodeoxycholic acid, deoxycholic acid and ursodeoxycholic acid (UDCA, DCA, CDCA, m/z391.2) to be significantly higher in the colon but not duodena of the HT group compared to the $\mathrm{HC}$ rats (Figure 16c). This observation suggests that the reuptake of these bile acids is inhibited by the LE diet, likely by soyasaponins. However, lithocholic acid (LCA, m/z 375.3) and cholic acid (CA, m/z 407.0) do not discriminate between the groups.

Taurodeoxycholic acid and/or taurochenodeoxycholic acid (TDCA, TCDCA, m/z 498.3), 3-keto-taurocholic acid (3keto-TCA, $m / z$ 512.3) and taurocholic acid (TCA, $m / z 514$.3) were all discriminative ( $A \cup C \geq 0.748$ ) between HC_Colon and HT_Colon, with higher levels in control animals (Figure 17). These bile acids were also discriminative between the colon and duodenum of the $\mathrm{HC}$ group, suggesting that they undergo modifications while traveling through the digestive tract. As seen in Figure 17b, taurocholic acid ( $\mathrm{m} / \mathrm{z} 514.3)$ is mainly present in the HC_Colon group. It is clear that these three molecular species that are taurine-conjugated bile acids have a strong discrimination power between HC_Colon and HT_Colon and are with a higher amount present in the HC_Colon image (Figure 17b). N-arachidonoyl taurine is a polyunsaturated fatty acid (PUFA) conjugated with a taurine group that acts in the brain as a neurotransmitter and plays a role in insulin secretion in the pancreas $[118,119]$. $\mathrm{N}$-arachidonoyl taurine is discriminative (AUC > 0.750) between $\mathrm{HT}$ _Colon and HC_Colon (Figure 17a), being prevalently present in the HT_Colon. 


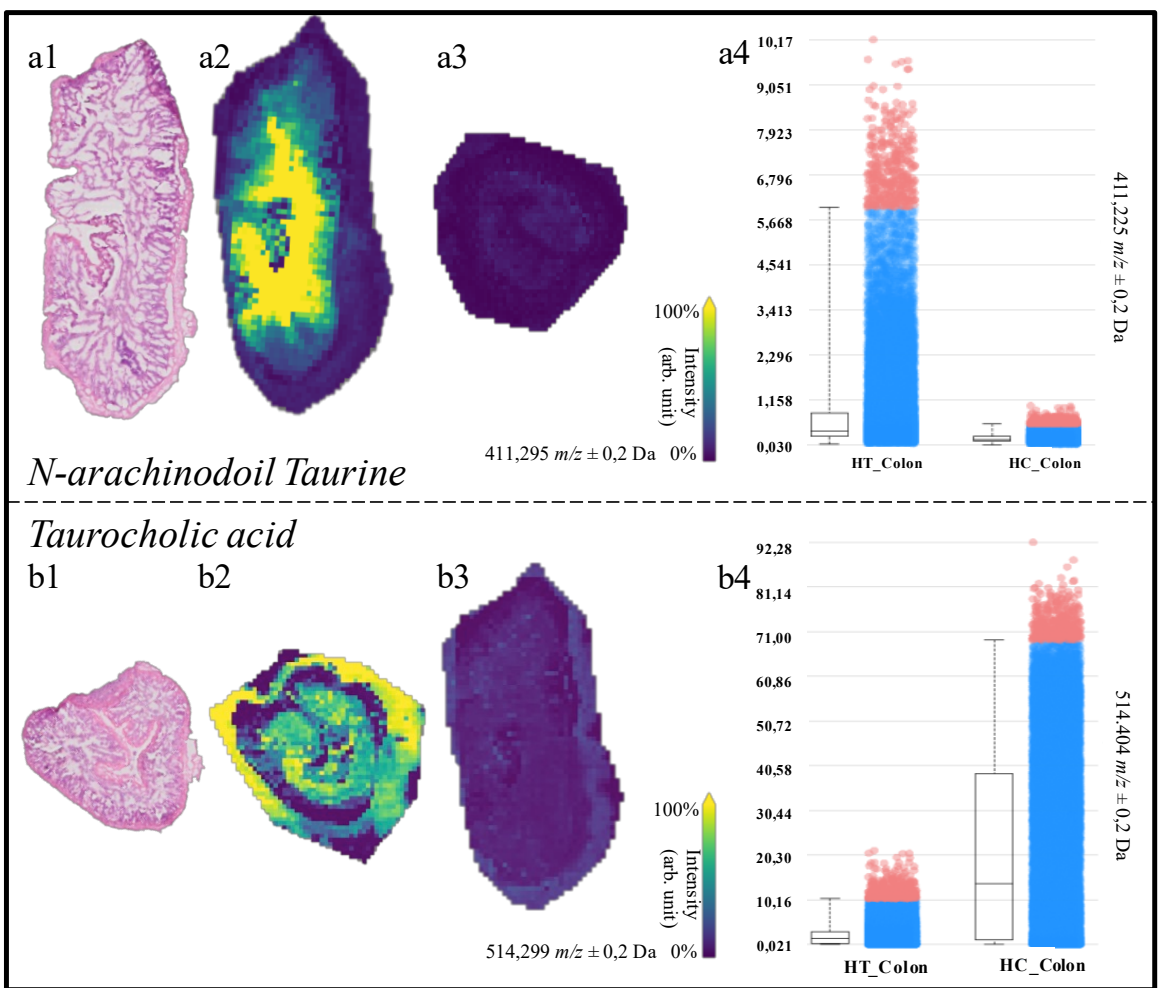

Figure 17 - [a1-4] Molecular images of N-arachinodoil Taurine in the HT-colon (a2) and HC-colon (a3). a4 shows a boxplot of the intensity of $\mathrm{N}$-arachinodoil taurine in the HT-colon vs. the HC colon. [b1-4] Molecular images of Taurocholic acid in the HC-colon (b2) and HT-colon (b3). b4 shows a boxplot of the intensity of $\mathrm{N}$-arachinodoil taurine in the HT-colon vs. the HC colon.

Deconjugation of taurine-conjugated bile acids is carried out by the gut microbiota, namely by the following bacterial genera: Bacteroides, Bifidobacterium, Clostridium, Lactobacillus, and Listeria [120]. Deconjugation occurs by bile salt hydrolase (BSH) enzymes, which are highly present in Bifidobacterium (Figure 18). The end products of this reaction are free taurine and deconjugated bile acid which were detected in the present study. Our results correlate with the work of Micioni Di Bonaventura et al. who indicated an increase of the Bifidobacterium in the LE treated group [45]. A previous study demonstrated that dietary taurine supplementation reduced serum cholesterol levels by increasing the activity of hepatic cholesterol 7ahydroxylase (CYP7A1), which is involved in the process of converting cholesterol into bile acids and increases the fecal excretion of bile acids in hypercholesterolemic rats [121]. The deconjugation of the taurine-conjugated bile acids by the Bifidobacterium 
will act similarly to taurine supplementation, as more of the taurine will come free and will be reabsorbed before it reaches the colon by the taurine transporter [122] (Figure 18). Taurine also plays a role in elevating LDL receptor levels, which results in lower cholesterol serum levels [123]. When the results of the increased bile acids excretion (CDCA, DCA and/or UDCA) and the decreased taurine-conjugated bile acids (TDCA/ TCDCA, 3keto-TCA and TCA) in the HT group are combined with the findings from Micioni di Bonaventura et al., we can conclude that the taurine-conjugated bile acids lost their taurine (TDCA/ TCDCA) and that the other bile acids (DCA/CDCA) end in the feces. This can be attributed to the prebiotic activity of LE rich in soyasaponins. Although we did not detect the molecules responsible for the hypocholesterolemic effect (soyasaponin I and $\beta g$ ), we prove here that MALDI-MSI allowed the understanding of their effects in the intestines (Figure 18).

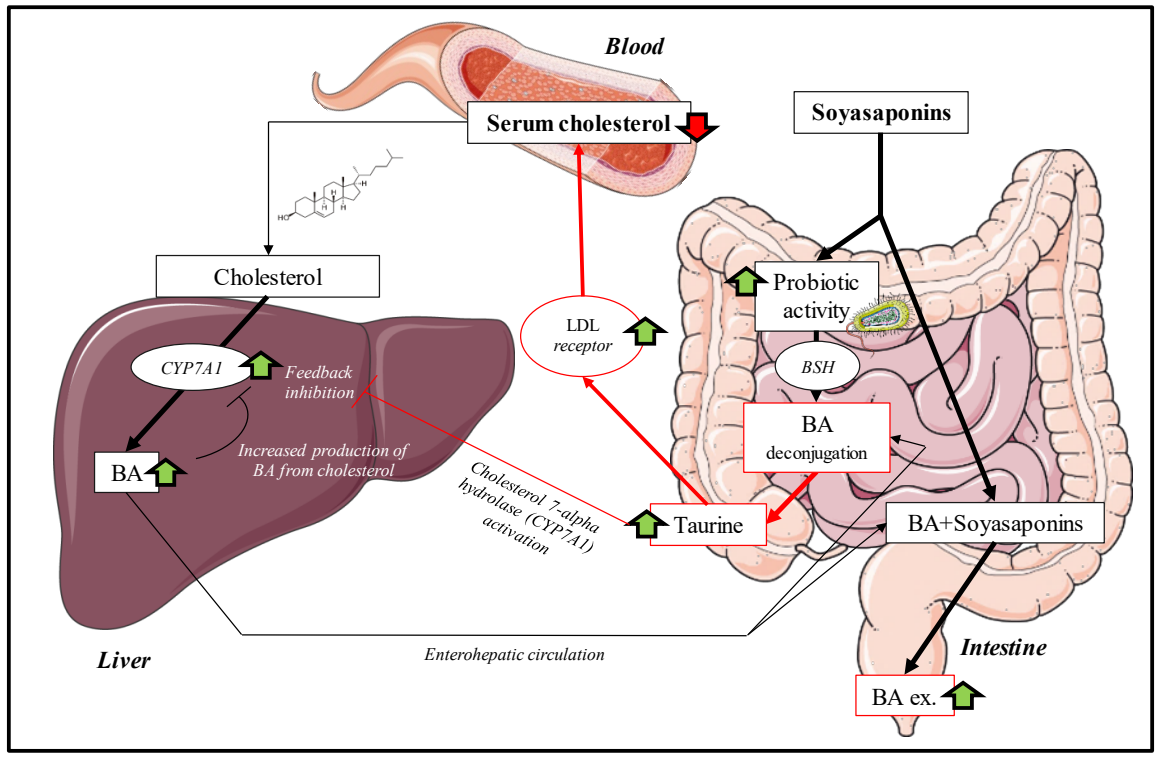

Figure 18 - Overview of the cholesterol-lowering mechanisms of soyasaponins by increasing the excretion of BA with the faces, which stimulates the production of new BA from cholesterol. Increased probiotic activity results in an increased release of free taurine. Taurine both increases the activity of the Cholesterol 7-alpha hydrolase enzyme in the liver (CYP7A1) and stimulates LDL receptors in the body, resulting in an overall reduction of serum cholesterol. 


\subsubsection{Investigation of cholesterol and lipid modifications induced on the brain by the LE treatment}

The PLSAs and PCAs for the whole brain sections and sub-sections of the three experimental groups $(\mathrm{C}, \mathrm{HC}, \mathrm{HT})$ did not show statistically relevant differences (Figure 19b). Additionally, the peak related to cholesterol sulfate and connected molecular images of the distribution of cholesterol in the brains showed differences between different animals but not related to different groups. (Figure 19c/d). The caudate putamen, hippocampus, prelimbic cortex and cerebellum (white and grey matter), were investigated due to their connection to memory deficit and possible changes in the hippocampal phenotype associated with hypercholesterolemia and obesity $[114,115]$. Preliminary behavioral tests performed from Micioni Di Bonaventura et al. showed no significant behavioral change among the three experimental groups [45]. Here, No significant differences were found in cholesterol or lipid composition in these sub-regions. As previous studies have only shown an association between hypercholesterolemia, obesity and memory deficits, it seems reasonable that there are no relevant results from the behavioral tests. These results indicate that neither the high-cholesterol diet nor LE treatment led to significant molecular variations in the brain lipid composition and distribution in rats observable with MALDI-MSI.

The absence of lipid and cholesterol changes in the brain is due to various factors. Studies showed that a high-cholesterol diet could induce relevant increases in plasma and liver cholesterol, but not brain cholesterol levels [124], in line with our observations. Additionally, the duration of the high-cholesterol diet and the treatment was too short to lead to concrete changes in brain lipid composition. This speculation is based on previous knowledge that total cholesterol absorption, synthesis and turnover is strongly correlated with body fat composition and higher in obese subjects compared to non-obese subjects [125]. It is worth noting that the rats were sacrificed in non-obese conditions. 


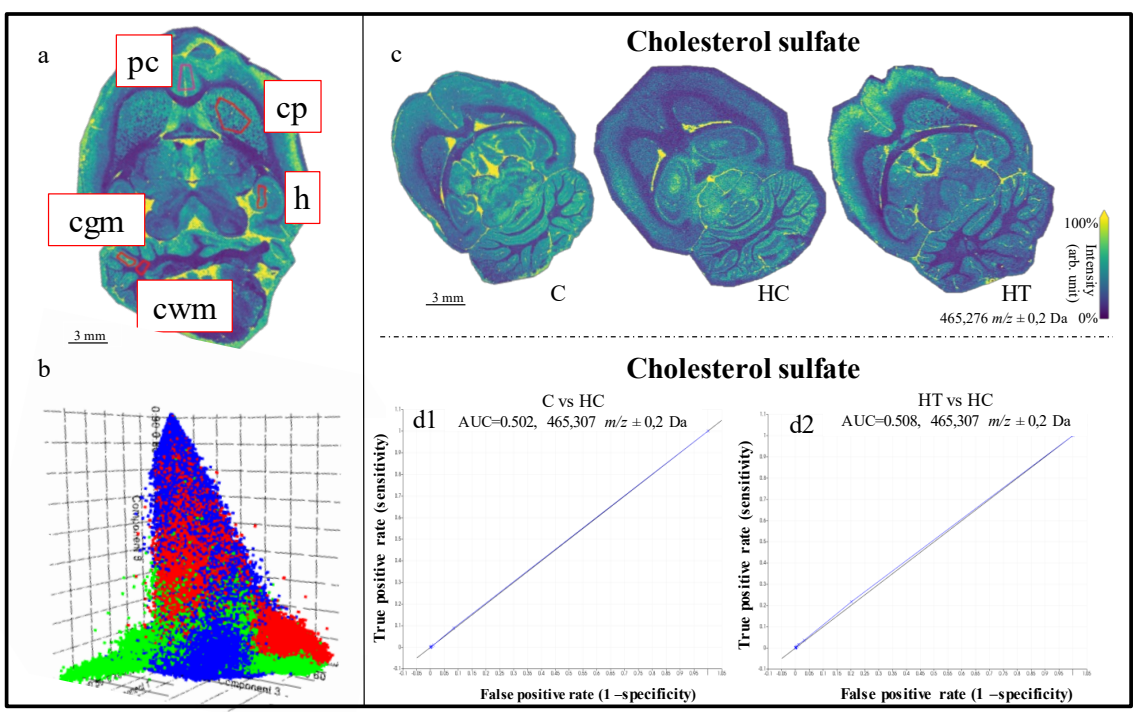

Figure 19 - [a] Molecular images of the brain with highlighted in red, the regions of interest (RO/s) delineated for the analysis: prelimbic cortex (pc), caudate putamen (cp), hippocampus (h), white (cwm) and grey (cgm) matter in the cerebellum. [b] 3D loading plot for the pLSA of the whole brains of the three groups. Blue represents the control group (C), green the hypercholesterolemic control (HC), and red the hypercholesterolemic treated group (HT). Results display no separation among the different groups. [c] Molecular images based on the distribution of cholesterol sulfate $(\mathrm{m} / \mathrm{z}$ 465,3) over the whole brain for the C, HC and HT groups. [d1-2] ROC plots for the cholesterol sulfate (m/z 465.3) from the comparison between (d1) $\mathrm{C}$ and HC, and between (d2) HT and HC.

Furthermore, in healthy brains, the several mechanisms involved in the maintenance of brain homeostasis (e.g., apolipoprotein E, ABC transporters) actively regulate the level of cholesterol in the brain by blocking its entrance and activating or blocking secondary pathways for cholesterol synthesis [126]. This led to conclude that the hypercholesterolemic treatment did not affect the brain lipid composition and distribution within the timeframe of the study. 


\subsection{Chapter conclusions}

The present study employed MALDI-MSI to investigate modifications in cholesterol, lipid and bile acid metabolism in rat liver and intestine as well as cholesterol and lipid composition in rat brain for a better understanding of the effect of a treatment with a cholesterol-lowering, hydro-alcoholic LE containing soyasaponins. We demonstrated that the LE does not modify the cholesterol and lipidic content of the brain nor the cholesterol composition in the liver. On the other hand, in the liver, we highlight the presence of a lipid (PG 36:4) connected with steatosis in untreated hypercholesterolemic rats.

The results from tissue analysis correlate with previous findings as reported by Micioni Di Bonaventura et al. We demonstrated that some BA (UDCA, DCA, CDCA, m/z 391.2) are not reabsorbed in the intestine after LE treatment. Additionally, we discovered that the hypocholesterolemic effect of LE treatment rich in soyasaponins is not only due to the increased excretion of BA with the feces (which forces the animal to produce new BA from cholesterol), but also to the prebiotic effect of LE. This led to an increased hydrolyzation of taurine conjugated bile acids from the gut microbiota, resulting in free taurine. Taurine has an upregulating effect on hepatic cholesterol 7ahydroxylase and LDL receptors leading to a reduction of the total cholesterol levels in the blood.

We here demonstrated that MALDI-MSI is a valuable tool to investigate and locate several molecular changes in different tissue types leading to a better understanding of the mechanisms behind the cholesterol-lowering treatments. Specifically, an untargeted technique such as MALDI-MSI led us to the discovery of the multi-organ/synergistic biological effects of LE providing a better insight of its cholesterol-lowering properties in rat models. 



\section{Chapter 4}

FFPE tissue-based quality control for MALDI-MSI analysis of tryptic

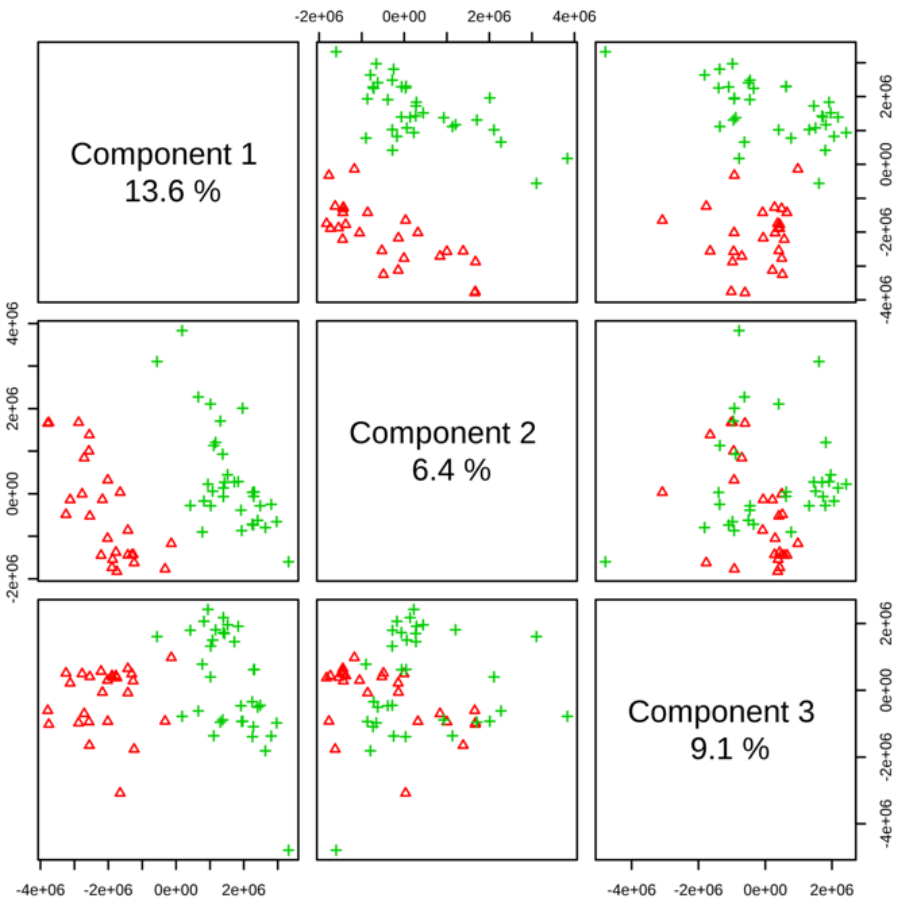

Adapted from:FFPE tissue-based quality control for MALDI-MSI analysis of tryptic peptides and application to predict neoadjuvant therapy response in breast cancer

Caro Thomassen**, Michele Genangeli**, Renée Granzier, Jo Capell, Fanny Boyaval, Loes Kooreman, Briete Goorts, Ron M.A. Heeren, Marjolein Smidt, Tiffany Porta Siegel

Article in Preparation

${ }^{* *}$ shared first authorship 


\section{$4 \quad$ FFPE tissue-based quality control for MALDI-MSI analysis of tryptic peptides}

\subsection{Abstract}

Matrix-assisted laser desorption ionization (MALDI) mass spectrometry imaging (MSI) is gaining momentum as a discovery tool in the clinical oncology field.

Breast cancer $(\mathrm{BC})$ is the leading cause of cancer among women worldwide with an annual increase. Despite research efforts, no available tool can accurately predict tumor response to treatment; therefore, surgery is never omitted. Worldwide, millions of formalin fixated and paraffin embedded (FFPE) $\mathrm{BC}$ tissues are available in pathology departments. FFPE protocols for MSI analysis have been optimized, however imaging tryptic peptides remains challenging due to experimental complexity.

Key aspect of this research is the development of a quality control (QC) based on tissue samples which undergo the same sample preparation as the BC samples. In this paper, we used a QC tissue made from FFPE-pork fat and we compared it to cytochrome $\mathrm{C}$, to monitor the variability and reproducibility of the clinical BC cohort. We then applied the strategy to 54 clinical ductal carcinoma BC samples to predict response to NST. The pork fat was an accurate quality control in 98\% for data selection, while cytochrome C was accurate only in $65 \%$ of the measurements. Based on our data we can state that pork fat is suitable as a QC for tryptic digestion experiments and more able to mimic the tissue digestion compared to cytochrome C. After removing outliers in the BC data based on the pork fat as QC, separation of $\mathrm{pCR}$ and non-pCR in $\mathrm{BC}$ tissue was possible. At least four peaks indicate to be discriminative between pCR and non-pCR in BC tissue. Further investigation into those peaks is necessary. Separating $\mathrm{PCR}$ and non-pCR in BC subgroups was only successful in the triple negative BC (TNBC) and ER-HER2- subgroup. 


\subsection{Introduction}

Over the past two decades, mass spectrometry imaging (MSI) has become an important analytical technique in clinical research [127]. Particularly, matrix-assisted laser desorption/ ionization (MALDI) - time of flight (TOF) MSI has been successfully applied in clinical oncology to investigate tumor molecular signatures (i.e. from proteins, lipids, peptides, and carbohydrates) in pancreatic cancer, colorectal cancer and breast cancer (BC), just to name a few [128]. Insights provided additional value compared to routine histopathological evaluation for tumor margins, grading and prognosis [128]. This enabled to tackle current clinical challenges where confounding factors or lack of sensitivity and specificity renders the diagnostics or prognostics very difficult to establish with routine examination (e.g. magnetic resonance imaging [MRI] and/or immunohistochemical staining [IHC]) [129]. Previous studies have proven the ability of MSI to predict treatment response in triple negative breast cancer (TNBC) [130]. Seeley and Caprioli et al. demonstrated a set of six proteins differentially expressed in the primary BC tumor that could predict lymph node metastasis with an accuracy higher than 70\% [129] and Cornett et al., demonstrated its ability to distinguish invasive- from in-situ carcinomas [131]. Yet, no study has investigated the potential of MSI to predict response to neoadjuvant therapy in breast cancer. BC is the leading cause of cancer among women worldwide [132]. However, tumor response to therapy differs considerably, it ranges from the pathologic complete response (pCR) to non-response, with the progression of the disease at worst [133-135]. Predicting pCR non-invasively in breast cancer after neoadjuvant systemic therapy (NST) is still a big challenge as it can be hard to visualize the tumor. Therefore, surgery is never omitted even when MRI shows a pCR [136].

Worldwide, millions of tissues of various diseases including BC are available in tissue biobanks from pathology departments. These tissues are mostly formalin fixated and paraffin embedded (FFPE) and are accompanied by well documented information about patient treatment, response, disease progression and other 
relevant data [128]. Protocols have been optimized to make FFPE amenable for investigation by MSl; which therefore can provide spatially localized molecular information that are correlated with pathological and morphological features of tissue sections. However, the entire process of imaging tryptic peptide and subsequent protein identification remains challenging mainly due to its experimental complexity, where even small differences between sample preparation and handling can cause variability in the results. Sample handling and preparation is of upmost importance to ensure spectral quality and reproducibility [128]. In the past years, several research groups have worked on strategies to improve the quality and detection of peptides and proteins, however, none of these experimental set-ups made use of a proper quality control (QC) sample $[128,137,138]$.

Cytochrome $\mathrm{C}$ as a single protein spotted near the samples has been used to monitor digestion efficiency $[139,140]$ and normalization steps are generally applied to account for the experimental variation arising from day-today fluctuations in sample preparation and measure instrument performance [128].

QC samples are of importance in monitoring analyte technical variability, on-tissue trypsin digestion efficiency and reproducibility issues which can occur if samples are measured on different days where different humidity and temperatures may influence the results. Variable conditions that are technically unavoidable for clinical cohorts' analysis. Uncontrolled temperature and humidity can lead to variations in sample preparation, and ultimately variations in the results generated [140]. Further development of a quality control for ontissue digestion was also strongly recommended by the round robin study recently published in 2018 by Buck and coworkers [141]. Still, suitable methods able to monitor the trypsin digestion efficiency directly on tissue is to our knowledge not been published yet. 
A key aspect of the present research is to develop an adequate QC based on tissue samples designed to undergo sample preparation simultaneously to our clinical samples and therefore monitor on-tissue trypsin digestion, experimental variance and reproducibility. In this paper, we used FFPE tissue made from pork fat and we compared the signal measured within days and in-between days to those measured from cytochrome C (spotted next to the tissue before matrix application), to monitor the variability and reproducibility of the clinical BC cohort.

The strategy was then applied to clinical BC samples to predict pCR after NST [142]. We monitored the digestion efficiency and possible technical variability of the QCs over the 58 days of analysis; which was used to exclude outliers from the multivariate analysis of our clinical cohort. With this strategy, we were able to reveal molecular (peptide) patterns able to classify patient according to their response to treatment.

\subsection{Experimental section}

\subsubsection{Chemicals and reagents.}

Xylene was purchased from Honeywell, NY, USA. Hexane, ethanol, methanol and water were purchased from Biosolve (Valkenswaard, The Netherlands). Citric acid, Octyl-a/b glucoside 10 mM solution, ammonium bicarbonate, alpha-4-cyanohydroxycinnamic acid (CHCA), cytochrome C, Trypsin, Hematoxylin Gill II, Eosin 0,2\% alcoholic solvent and Entellan were purchased from Merk (Darmstadt, Germany).

\subsubsection{Study design.}

In this research, female patients ( $n=27$, age $>18$ ) with invasive ductal carcinomas (tumor size $\geq 2 \mathrm{~cm}$ and/or lymph metastasis) received 24 weeks of chemotherapy and/or immune therapy before surgery i.e. neoadjuvant systemic therapy (NST). Patients received different NST, based tumor characteristics. Patients 
with HER2+ tumors received neoadjuvant intravenous trastuzumab and chemotherapy. Patients with HER2-tumors received neoadjuvant chemotherapy. The therapy consisted of four cycles of doxorubicin and cyclofosfamide every three weeks. ER + and/or HER2+ patients received an additional four cycles of docetaxel every 3 weeks. Triple negative patients received twelve cycles of weekly paclitaxel. Biopsies were obtained during ultrasound before NST and immediately fixed in formalin and embedded in paraffin.

All patients underwent surgery after NST. Pathologists assessed the pathologic response on the removed tumor section (Table 18). Accordingly to the Pinder Classification system, tumors were classified as pCR when no evidence of invasive residual tumor was found, but DCIS can be present. The non-pCR tumors had no evidence of response to therapy. All other tumors were classified to partial responders (pPR). 


\begin{tabular}{|c|c|c|c|c|c|c|c|}
\hline \multirow[b]{2}{*}{ Patients $(n=27)$} & \multirow[b]{2}{*}{ cTNM } & \multirow[b]{2}{*}{ урTNM } & \multirow[b]{2}{*}{ pCR yes/no } & \multicolumn{4}{|c|}{ Receptors $(0=$ neg; $1=$ pos $)$} \\
\hline & & & & ER & PR & HER2 & Age \\
\hline 1 & cT2N1 & урт0Мо & Yes & 0 & 0 & 1 & 58 \\
\hline 3 & $\mathrm{cT} 2(\mathrm{~m}) \mathrm{N1a}$ & ypTONO & Yes & 0 & 0 & 0 & 43 \\
\hline 5 & cT2mN1M0 & ypT1cN0 & No & 1 & 1 & 1 & 47 \\
\hline 6 & cT3N2 & ypT1aN1a & No & 0 & 0 & 0 & 54 \\
\hline 7 & cT2NOMO & yрT2N2a & No & 1 & 1 & 0 & 58 \\
\hline 8 & cT3N+ & урT2N2a & No & 1 & 0 & 0 & 73 \\
\hline 9 & cT4N2 & ypT4bN1a & No & 0 & 0 & 1 & 72 \\
\hline 10 & cT3N1 & урT2N2a & No & 0 & 0 & 0 & 63 \\
\hline 13 & cT3NO & ypTONO & Yes & 1 & 0 & 1 & 37 \\
\hline 14 & cT3N1 & урT2NO(i+) & No & 1 & 0 & 0 & 60 \\
\hline 15 & cT1NO & ypT1 & No & 1 & 1 & 0 & 50 \\
\hline 16 & сT3mN1 & ypT1aN1a & No & 0 & 0 & 0 & 44 \\
\hline 17 & cT2NO & yрT2NO & No & 0 & 0 & 0 & 53 \\
\hline 18 & CT3NO & yрT2NO & No & 1 & 0 & 0 & 70 \\
\hline 19 & cT3N1 & ypT2NO & No & 0 & 0 & 0 & 40 \\
\hline 20 & cT3N1 & ypTO NO & Yes & 0 & 0 & 1 & 48 \\
\hline 21 & cT2N1 & ypT1a NO & No & 1 & 1 & 0 & 70 \\
\hline 22 & cT2N1 & ypTO NO & Yes & 1 & 0 & 1 & 49 \\
\hline 23 & cT1N1 & ypT1cN1 & No & 0 & 0 & 0 & 68 \\
\hline 24 & cT2N1 & ypTONO & Yes & 1 & 0 & 1 & 45 \\
\hline 25 & cT2NO & ypT1cNx & No & 1 & 1 & 0 & 44 \\
\hline 26 & cT2mNO & ypT1aNO(i+) & No & 1 & 0 & 0 & 33 \\
\hline 27 & cT2NO & ypT1aNO & No & 0 & 0 & 1 & 48 \\
\hline 28 & cT2NO & ypT1cNO & No & 1 & 1 & 0 & 59 \\
\hline 29 & cT2mN1 & ypT1cN0 & No & 0 & 0 & 1 & 51 \\
\hline 30 & cT2N1 & ypTONO & Yes & 0 & 0 & 0 & 41 \\
\hline 31 & cT3NO & ypTONO & Yes & 1 & 0 & 1 & 53 \\
\hline
\end{tabular}

Preliminary tumor measurements were obtained by physical examination, mammography and ultrasound. In case of a deviation between measurements, the largest tumor size was held. Biopsies obtained during ultrasound examination (or in some cases stereotactic via MRI) underwent further examination by pathologists to determine tumor grade, hormone (ER + PR) and human epidermal growth factor 2 
(HER-2) receptor status. The tumor was found to be hormone positive in $>10 \%$ of the cases staining in immunohistochemical (IHC) assays (Table 19).

Several sections from each patient biopsy were analyzed either once, in duplicate or triplicate with MALDI-MSI.

\begin{tabular}{|c|c|c|}
\hline & \multicolumn{2}{|c|}{ Patients total $n=27$} \\
\hline & $\mathrm{pCR} n=8(30 \%)$ & non-pCR $n=19(70 \%)$ \\
\hline Mean age years & 47 & 57 \\
\hline \multicolumn{3}{|l|}{ NST } \\
\hline - $\quad$ Only chemotherapy & $3(11 \%)$ & $15(56 \%)$ \\
\hline - $\quad$ Chemotherapy + immunotherapy & $5(19 \%)$ & $4(15 \%)$ \\
\hline Tumor $0-2 \mathrm{~cm}$ pre NST & $1(4 \%)$ & $3(11 \%)$ \\
\hline Tumor $>2 \mathrm{~cm}$ pre NST & $7(26 \%)$ & $16(59 \%)$ \\
\hline ER-positive & $4(15 \%)$ & $10(37 \%)$ \\
\hline HER2-positive & $6(22 \%)^{* *}$ & $(15 \%)$ \\
\hline \multicolumn{3}{|c|}{$\begin{array}{l}{ }^{*} \text { Categorical numbers in value (\%) per total } \\
{ }^{* *} \text { Patient } 1 \text { was HER2+ but did not receive immunotherapy. }\end{array}$} \\
\hline
\end{tabular}

\subsubsection{Tissue-based quality control for MSI procedures.}

Pork chops was obtained from a local grocery store, formalin-fixated and paraffin embedded. Two different pork fat sample blocks (referred to as "Pork fat 1 " and "Pork fat 2") were then sectioned with a microtome at $5 \mu \mathrm{m}$ and mounted onto clean conductive (5 $\Omega$ ) indium-tin-oxide (ITO) slides next to the clinical BC samples, prior to sample preparation. Prior MSI analysis, the pork fat underwent identical sample preparation step as the breast samples; as detailed in the next paragraph. Pork fat samples were prepared and measured over a period of 58 days together with the breast samples. 


\subsubsection{Breast samples and pork fat FFPE tissue preparations for MSI analysis.}

FFPE were cut into 5 mm tissue sections and mounted onto clean ITO slides

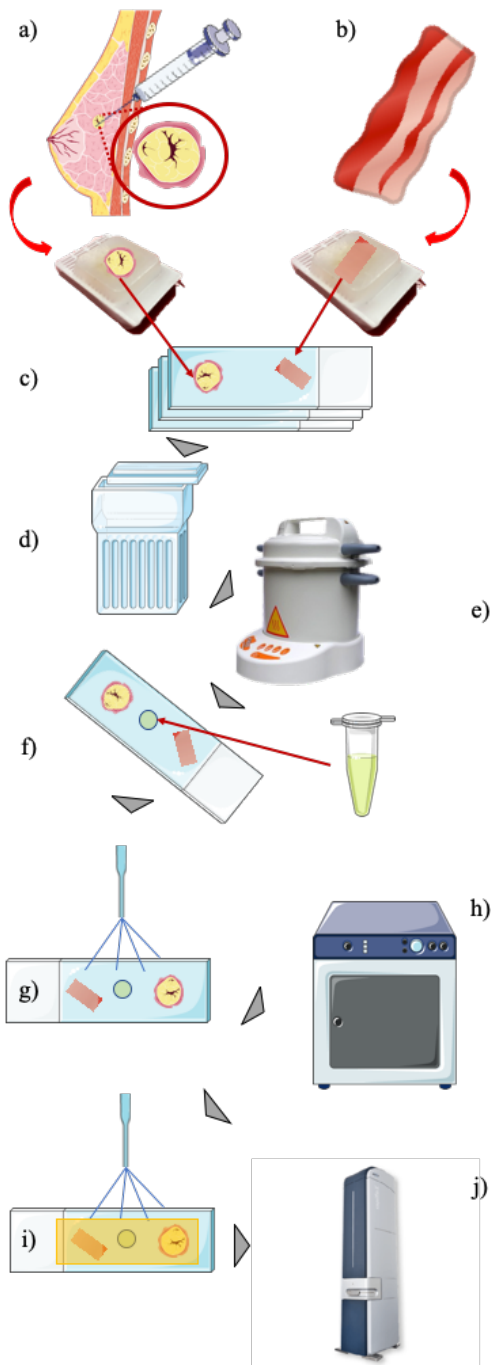
(Figure 20). The slides were cleaned in a sonicated bath of n-Hexane for 6 min, followed by another sonicated bath in 100\% ethanol for 6 min. Afterwards, control sample pork fat was sectioned at $5 \mu \mathrm{m}$ mounted on the same ITO slides, next to the breast samples. Tissue samples were deparaffinized on a heating plate and by washing with xylene (100\%) first for 5 min, and then a second time for $10 \mathrm{~min}$. This was followed by rehydration with absolute ethanol twice for 2 min while shaking gently every 30 sec. Last, the samples were rehydrated with ultra-pure water twice for 5 min while gently shaking every 30 seconds. Antigen retrieval was performed in a controlled way (Retriever 2100) in a $10 \mathrm{mM}$ citric acid buffer ( $\mathrm{pH} \mathrm{6.0)}$ and for $20 \mathrm{~min}$. Samples were allowed to cool down inside the antigen retriever for 2 hours before being taken out and washed twice for 1 min with ultra-pure water. Subsequent on-tissue digestion was performed by spraying $0.04 \mathrm{\mu g} / \mathrm{mL}$ trypsin

Figure 20 - Schematic overview of the overall workflow - from tissue biopsy to peptide MALDI-MS experiment. (a) needle biopsy is taken from the breast tumor Subsequently, the resected tissue is formalin-fixated, and paraffin embedded (FFPE) (b). Pork fat is formalin-fixated, and paraffin embedded (c). FFPE samples are cut into $10 \mathrm{um}$ sections and mounted onto clean ITO slides (c). The samples undergo several washing steps (d) and antigen retrieval (e). Cytochrome C is spotted onto the slide after the Antigen retrieval step (f). Trypsin is sprayed onto the slide (g) and the samples are incubated overnight at 37.C (h). The slides are subsequently covered with CHCA matrix (i) and measured using the Rapiflex (i).

prepared with 10 mM ammonium bicarbonate and 100x octyl-a-bglucoside. 
Samples were then sprayed in a $10 \mu \mathrm{l} / \mathrm{min}$ format for 15 layers using the Suncollect (Sunchrom). Afterwards, the samples were incubated at $37^{\circ} \mathrm{C}$ for $16-$ 18 hours at a saturated environment using an in-house incubation chamber (50\% MeOH). Finally, the MALDI matrix CHCA (7 mg/mL in 50\% ACN + 0.2\% TFA was applied using the SunCollect as follow: the first layer at a flow rate of 10 $\mu \mathrm{L} / \mathrm{min} ; 2^{\text {nd }}$ layer at $20 \mu \mathrm{L} / \mathrm{min} ; 3^{\text {rd }}$ layer at $30 \mu \mathrm{L} / \mathrm{min}$ and $4^{\text {th }}-7^{\text {th }}$ layer at 40 $\mu L / \min$.

\subsubsection{MALDI-TOF-MSI analysis.}

All the MALDI-MSI experiments were performed on the Rapiflex ${ }^{\top M}$ Tissuetyper (Bruker, Bremen, Germany) operated in positive reflectron TOF mode in the mass ranges $\mathrm{m} / \mathrm{z}$ 600-3000. Images were acquired at a spatial resolution of $50 \times 50 \mu^{2}$ with a $10 \mathrm{kHz}$-laser frequency and 500 laser shots per pixel. Calibration was performed with Red Phosphorus. The BC tissue samples were analyzed in duplicate (unless otherwise stated) in a random order over 8 weeks to account for technical variation Additional triplicate measurements were acquired at a later stage of the research.

\subsubsection{Data pre-processing.}

Data was imported into flexImaging and co-registered to the H\&E stained images. Consequently, regions of interest (ROIs) were selected for the pork fat, cytochrome C, and BC samples. Next, tumor areas were annotated by a pathologist and then imported into SCiLs Lab software. Spectra were normalized against the total ion count (TIC) and mean spectra from all the tissue types (e.g. tumor, healthy tissue, pork fat, cytochrome C) were exported into mMass software [143] where baseline correction and peak picking were applied using a signal to noise ratio of 3. Additionally, trypsin and matrix related peaks were removed from the peak list. The generated peak lists (tumor, healthy tissue, pork fat, cytochrome C) were then imported into SCiLs Lab and 
used for further data analysis. From SCiLs Lab, the data was then imported into Metaboanalyst (Xia Lab, McGill, [144]) where extensive data analysis was performed.

\subsubsection{Data analysis.}

Principal component analysis (PCA) and probabilistic latent semantic analysis (pLSA) were performed on the TIC normalized spectra with a maximum of 10 components on defined regions of interest (ROI) using SCiLS Lab. PLSDA analysis was performed on the TIC normalized spectra on defined ROIs using MetaboAnalyst. Level and Pareto scaling methods were applied in order to emphasize the biological information in the data set and improve the biological interpretability [145]. Both the scaling methods provided comparable results. Results from the PCA on pork fat and cytochrome C in ScilsLab was used to filter the data and perform data analysis test on the BC samples (filtered-BC).

A supervised multivariate classification tool in Scils Lab was used to create a classification model [144]. The model was trained on several spectra from 19 BC samples (pCR and non-pCR) and validated on the remaining 17 samples. Only regions of interest were subjected to these tests.

Next, the filtered-BC data, as well as pork fat and cytochrome C data were uploaded in MetaboAnalyst where additional data analysis was performed, such as partial least square discriminant analysis (pLSDA) and volcano plot.

\subsubsection{Hematoxylin \& Eosin (H\&E) staining.}

The H\&E staining was performed after the samples were analyzed with the Rapiflex ${ }^{\top M}$ Tissuetyper. The matrix was gently removed with the usage of Kimwipe (Kimberly Clark) tissues, pre-dipped in methanol and then the sample rehydrated in ethanol for 3 min. each consecutively 100\% (2x), 96\% (2x) and 70\% (2x). The tissues were stained with hematoxylin for 3 min, washed with deionized water and stained with eosin for $30 \mathrm{sec}$. The tissue was washed under running tap-water and afterwards 
placed in absolute ethanol for 1 min, and fresh xylenes for 30 sec. Tissue sections were then covered with a cover glass and Entellan (glue). After staining the samples were dried at room temperature. High resolution optical images of the tissue samples were obtained with the MIRAX Desk Scanner (Zeiss, Oberkochen, Germany). 


\subsection{Results and discussion}

\subsubsection{Development of FFPE-based quality controls (QCs) for MALDI-MSI of tryptic peptides.}

Finding an adequate QC sample for the MSI workflow for trypsin digestion is challenging. This complication is even exacerbated with the preparation of formalinfixed paraffin embedded (FFPE) tissues that undergo several critical steps like paraffin removal by mean of harsh washing, antigen retrieval, trypsin digestion - illustrated in Figure 20. In our protocol, the antigen retrieval step was performed with an automated device (Retriever 1200) which led to some tissue loss for both QC and BC samples. Tissue loss can partly be assigned to the fatty composition of the tissue. Before the actual analysis of BC samples, a small optimization step was included to the protocol and tested on the pork fat samples. Those samples showed that incubating them overnight at $37^{\circ} \mathrm{C}$ after sectioning improved tissue adhesion to ITO slides, and therefore, reduced tissue loss after antigen retrieval.

Variation in data can also occur due to sample preparation steps and, as previous reported by Ly et al. (2019) [140], uncontrolled humidity. Deininger et al. [146] reports that while normalization strategies (e.g. TIC) can help in comparing expression levels across similar tissues, it does not account for heterogenous tissues and trypsin digestion efficiency. Recent studies have analyzed reproducibility of FFPE digestion followed by MSI analysis of human FFPE. Although those studies are sought mainly to evaluate and improve FFPE workflows, they proposed similar techniques to evaluate the dataset such as visual interpretation, pCA and pLSDA $[140,147]$. In addition, they proposed different controls for different purposes: Heijs et al., used an ROI with notissue to identify and remove matrix, trypsin and trypsin autolysis peaks which we used for resource in this study as well [148]. Additionally, control lists have been proposed for specific peak exclusion, such as trypsin and matrix related peaks which this research integrated as well. 
Another proposed control such as unpaired biopsy or human homogenous tissue FFPE can be also used but it is hard to obtain and distribute since ethical approval is necessary [149-151]. However, in clinical research there is a need for an established QC to measure day-to-day technical variability, and on-tissue trypsin digestion efficiency. An ideal QC sample should be analyzed simultaneously with the tissue specimen and undergo the same sample preparation procedure [152]. Because of these reasons, the development of a MALDI-MSI QC to account for those variations is of upmost importance.

Here we propose to use pork fat as a suitable tissue-based QC tissue, and compare it with cytochrome C. Pork fat was chosen because: i) it is easy to obtain and "non-precious" (i.e. not clinical); ii) it mimics the fatty composition of breast tissue while being homogeneous; and iii) it undergoes all sample preparation steps together with the breast tissues. Two blocks made of pork fat (referred to as Pork fat 1 and Pork fat 2) were FFPE prepared on different days to assess the variability and reproducibility of conceiving such tissues (Figure 20).

In the proposed experimental setup, each glass slide analyzed included a BC tissue, a pork fat tissue section and a spot of Cytochrome C (1 mg/mL) standard applied next to the BC tissue. It is worth noting that Cytochrome C was added after paraffin removal and antigen retrieval but before trypsin application; while pork fat tissues underwent every sample preparation step together with the BC-as depicted in Figure 20. Pork fat is a homogenous tissue which gives the advantage that, in case of tissue loss, homogenously distributed peaks are still present in the remaining tissue. Furthermore, we tested the reproducibility of the pork fat over 3 months and the signal from the pork fat tissue remained constant as shown in Figure 21. 

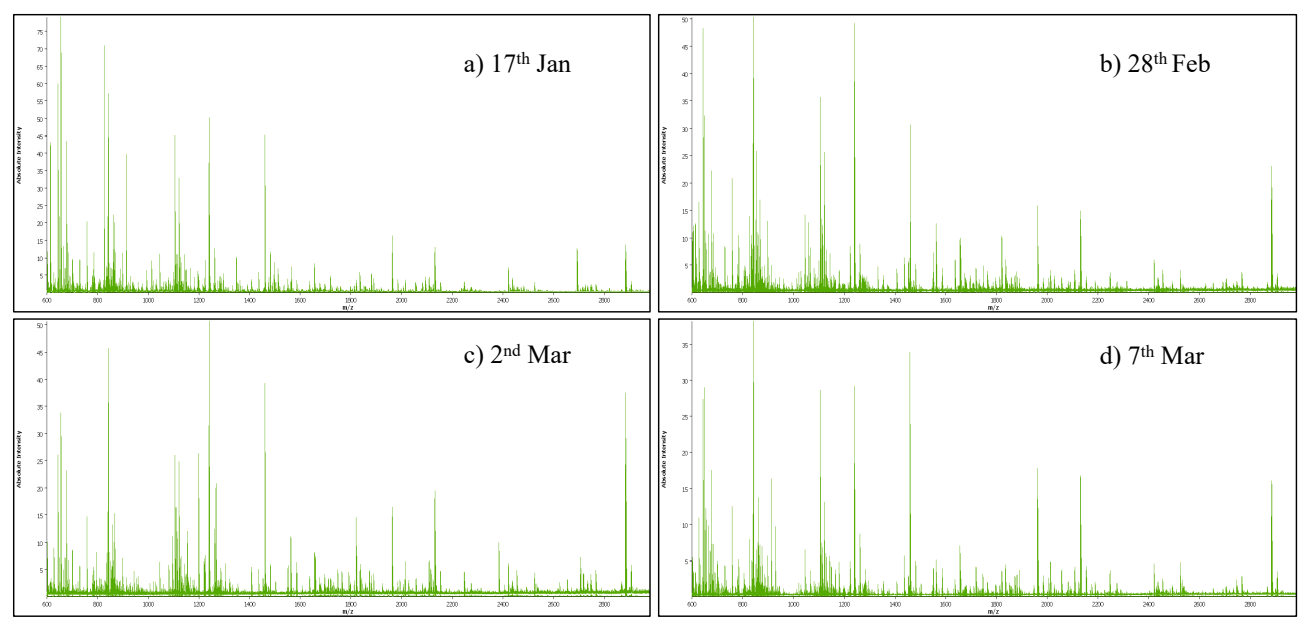

Figure 21 - Spectra from four different pork fat slices analyzed in different days. The signal is reproducible.

This allows to use the remaining pork fat tissue as a QC to remove outliers, even when tissue loss occurred (Figure 22).

An average spectrum from every QC tissue section was generated and visually assessed by two MALDI-MSI researchers independently, in terms of tissue digestion and spectra quality (Figure 22). A first evaluation of the spectra allowed to identify matrix cluster and/or trypsin-related peaks. In the case where matrix clusters are predominantly detected in the mass spectra (e.g. Figure 22) this is a clear indication that either the peptides were not extracted from the tissue during the sample preparation or the peptides were not digested. The results from the visual evaluations were compared and used as a reference point to discriminate between well and poorly digested tissues (BC tissue, pork fat and cytochrome C). 

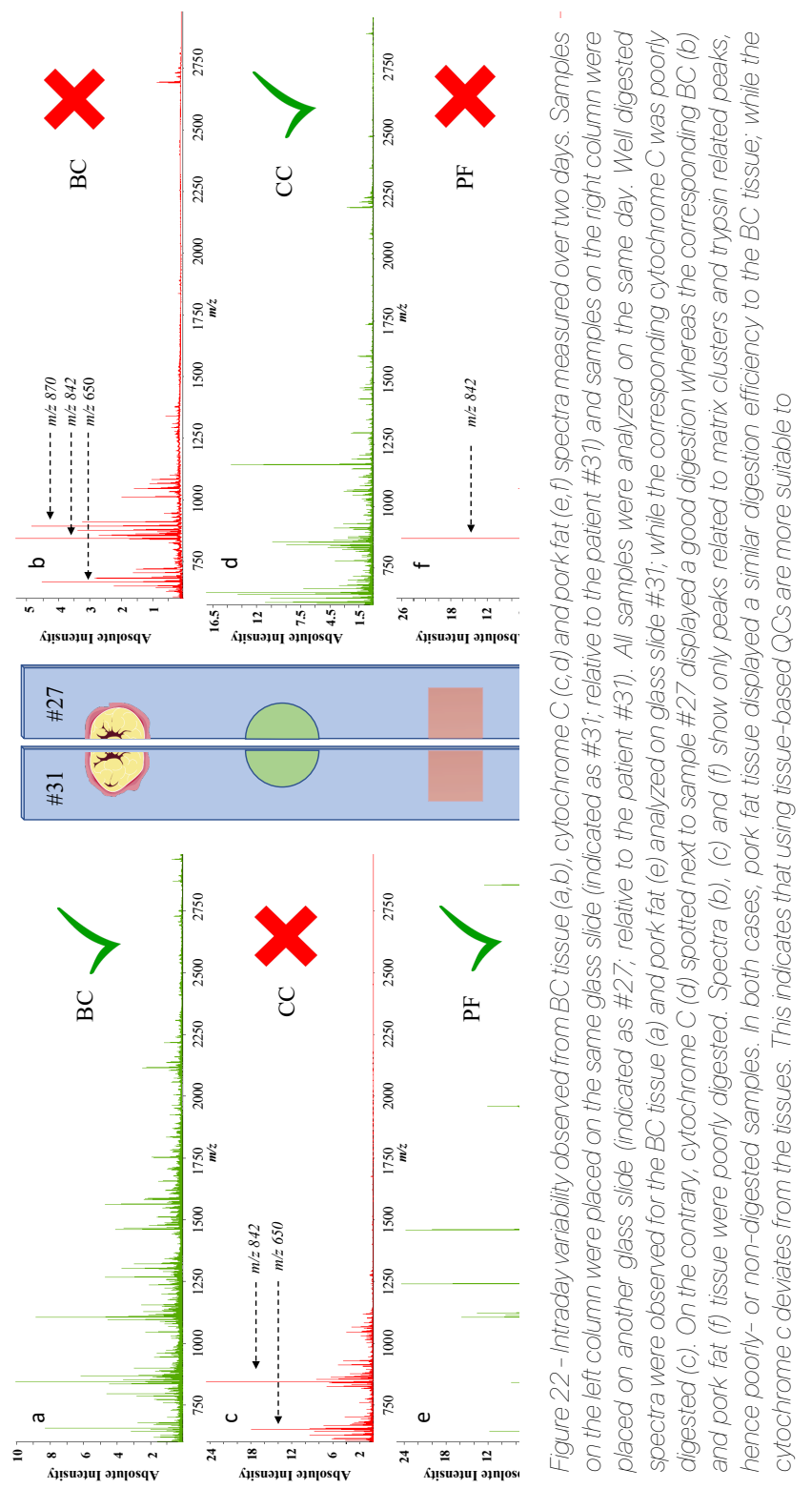

Preliminary results were then confirmed by multivariate analysis. The PCA and pLSDA on pork fat displayed complete separation between digested and undigested samples as shown in Figure 23. 


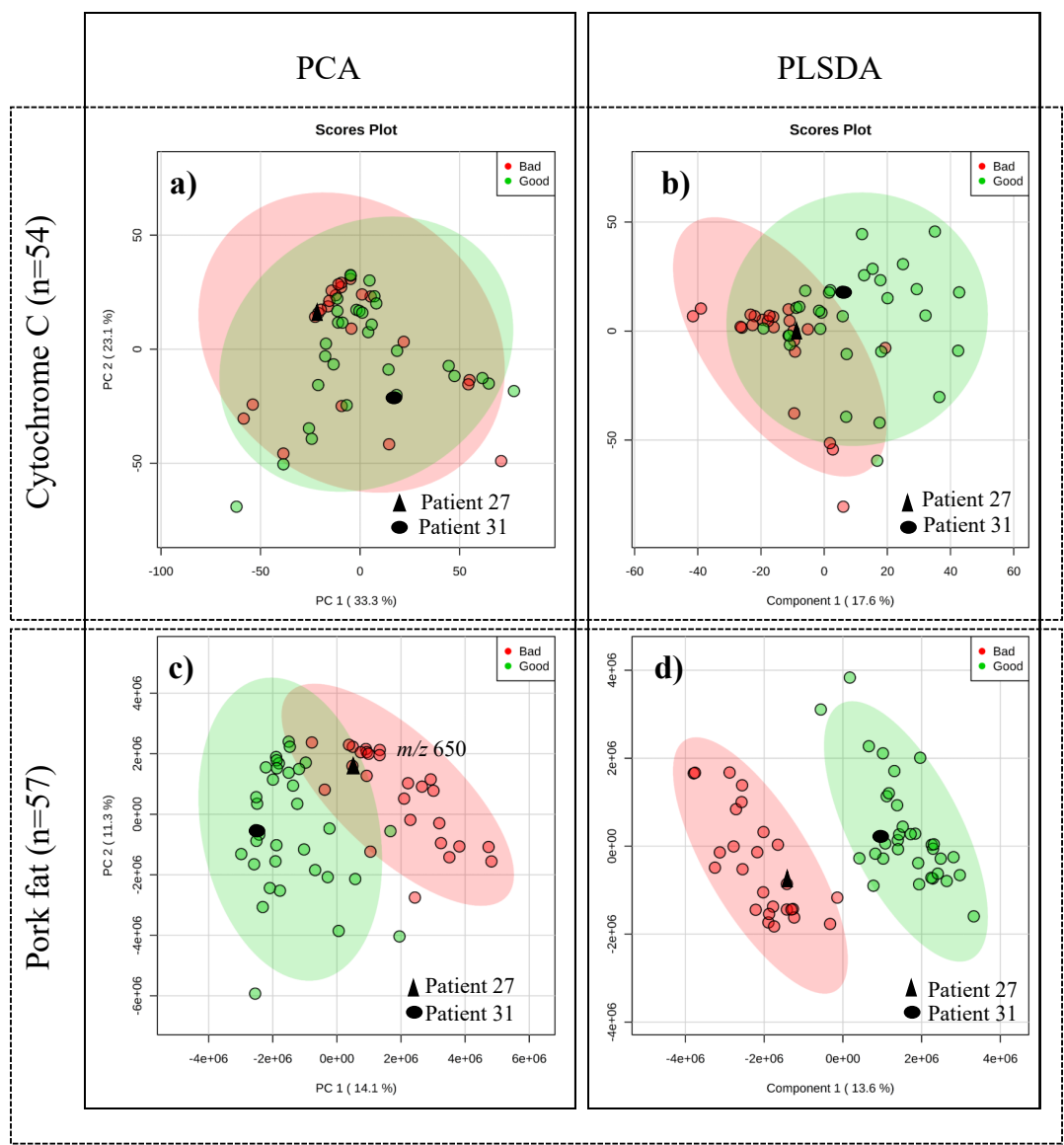

Figure 23 - PCA $(a, c)$ and PLSDA $(b, d)$ analysis of cytochrome $C(a, b)$ and pork fat $(c, d)$ data analyzed over a period of X days. Undigested samples are displayed in red (bad digestion) and digested samples in green (good digestion). PCA (a) and PLSDA (b) performed on the cytochrome C data were unable to separate the undigested from digested protein standards applied next to the tissues analyzed. PCA (c) and PLSDA (d) performed on the pork fat data show a complete separation between digested and undigested samples when using the PLSDA and a partial separation of the data when using the PCA. Corresponding spectra of the indicated patients can be found in Figure 4. In (a) and (b): $n=23$ undigested, $n=31$ digested. In (c) and (d), n=26 undigested, $n=31$ digested.

Components 1 and 2 accounted for $25.4 \%$ of the total variance between the two groups; and enabled to discriminate between well digested and bad digestion of pork fat tissues. On the contrary, there was no clear separation between digested and undigested cytochrome C standard.

We also compared the score between the digestion of pork fat, BC tissue and Cytochrom C within a day of analysis. As shown in Figure 22 and Figure 23, in most 
$(71 \%)$ of the cases, similar scores were obtained for pork fat and BC tissues while an opposite score for the cytochrome C. To illustrate, the average spectra from the sample slide analyzed on Day 3 (containing the BC tissue sample for patient 31) were scored as follow: 1) "good" digestion for the pork fat and BC tissues, 2) "bad" digestion of the Cytochrome C as the spectra recorded only displayed trypsin autolysis and matrix cluster-related peaks (Figure 22). As previously discussed, the fact that we could only detect the presence of matrix and trypsin-related peaks indicates that either the peptides were not extracted from the tissue during the sample preparation or the peptides were not digested at all.

The digestion efficiency displayed from every pork fat is comparable with the signal from the breast tissue on the same slide (Figure 22). When evaluating the entire dataset, we noticed that in 11 cases, the cytochrome $\mathrm{C}$ displayed an undigested spectra while the BC tissue and pork fat tissue contained digested peaks in the spectra. In 8 cases the cytochrome $\mathrm{C}$ resembled a digested spectrum while the BC tissue + Pork fat resembled a undigested spectra (Figure 24). In no cases the pork fat resembled undigested spectra while BC tissue contained digested peaks. In one case the pork fat resembled a digested spectrum while the BC tissue contained undigested peaks. As an example, the average mass spectrum measured for Patient 27 showed a good digestion score for the cytochrome $\mathrm{C}$ while a bad digestion for both the pork fat and the BC (only trypsin autolysis and matrix clusters peaks are present for the reasons mentioned above). On the other hand, the mass spectrum for patient 31 showed a good digestion score for the BC and pork fat, while a bad digestion for the cytochrome C (Figure 23). 


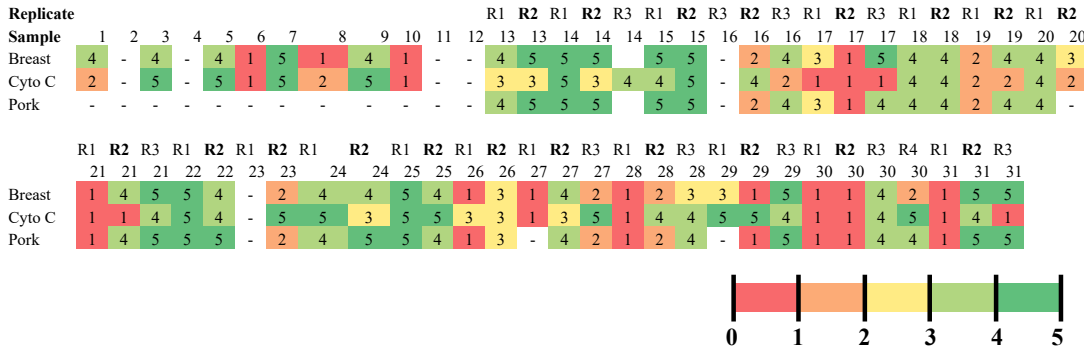

Figure 24 - Results from the double blinded visual examination of spectra from BC, Cytochrome C and pork fat. In most of the cases, the pork fat obtained a score similar or identical to the BC. The cytochrome C obtained random scores not linked to the other tissues.

An explanation for these observations is that the pork fat is an actual FFPE tissue sample (not a single protein) which undergoes every step of the sample preparation and is therefore able to mimic the BC sample in a much more efficient way than the Cytochrome $\mathrm{C}$. These findings demonstrate that solely using a single protein as QC is not enough to monitor the entire analytical workflow when preparing FFPE clinical samples.

For those reasons, we decided to filter the BC data based on the pork fat digestion score and not on the cytochrome C. In other words, we excluded outliers from the $\mathrm{BC}$ dataset when the pork fat samples were identified as outliers according to the PCA and pLSDA results of the pork fat spectra (Figure 22) - which validated our visual assessment.

\subsubsection{Application of the QCs to BC tissues Exclusion of BC data based on pork fat as QC}

In this study, the clinical cohort included nineteen female BC samples ( $\mathrm{pCR}$ and non-pCR) analysed in two technical replicates $(n=38)$. Eight additional samples which remained from a previous study were also included but were only analysed once due to the availability of the tissue.

A first PCA was performed on all data from the BC annotated tumor areas. Results showed that there is separation between the pCR and non-pCR patients (Figure 25) which is due to the high variance of the data. 

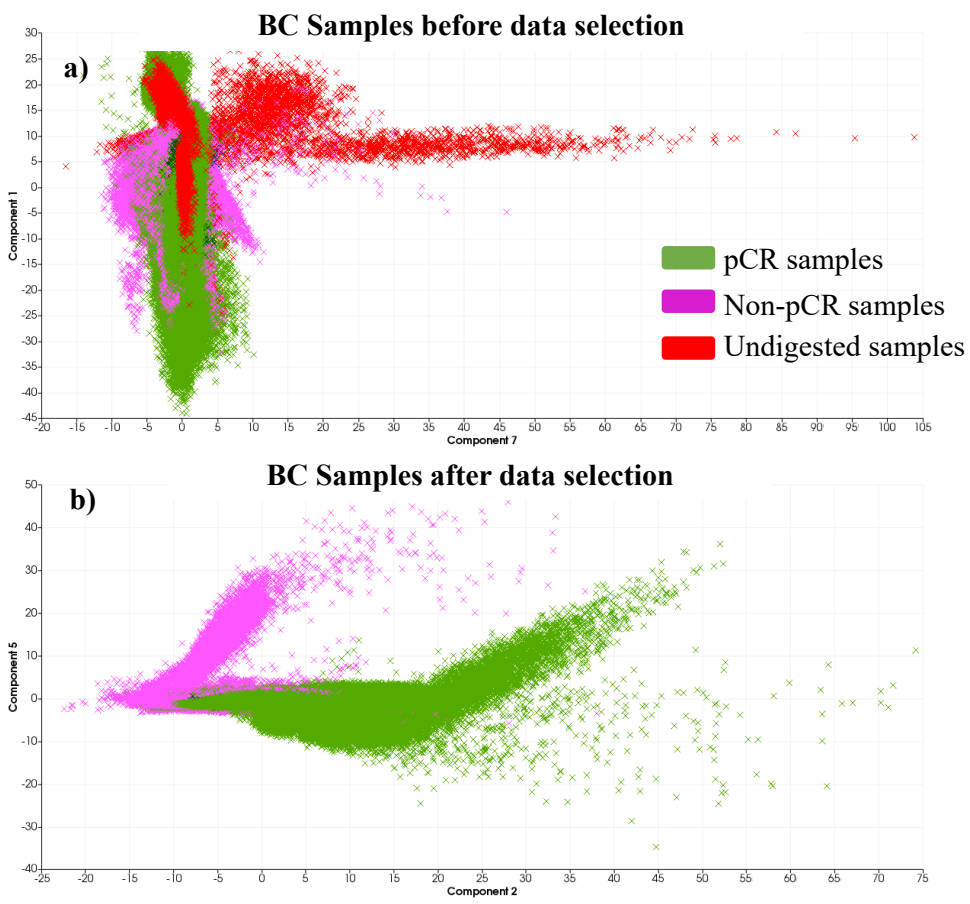

Figure 25 - PCA score plots displayed before (a) and after (c) selection of data for the multivariate analysis of the pCR I non-pCR BC data set. A PCA run at a pixel level of BC tissue before data selection (exclusion of outlier samples that were not properly digested) show a complete overlap of pCR and non-pCR samples and a partial separation of undigested samples (a). Removing the undigested samples based on the data selection from the pork fat as QC resulted in a partial, yet better separation of pCR and non-pCR samples (b). The separation of the two categories of sample was not possible prior exclusion of undigested samples.

After first visual inspection of the data, results display lower quality spectra; that could be due to poor digestion of the samples. Indeed, the BC tissue cohort is highly heterogeneous and due to local molecular environment, it is very likely that the digestion efficiency would differ from one sample type to another (e.g. tumor versus non tumor tissue). Therefore, it is very challenging to exclude samples solely based on visual and subjective inspection of the data as some regions might be less favourable to digestion. To ensure that the samples included in the statistical analysis of our clinical cohort only include good quality data and we excluded all samples for which a QCs sample (i.e. analysed simultaneously) was assigned as "bad quality" resulting from poor digestion. 
As a result, a total of 16 data were excluded out of the 38 tissue sections analyzed. An additional 9 samples from the excluded ones were re-analysed and a better digestion allowed to include them in the analysis. From the reanalysis, only one sample was excluded based on bad digestion criteria. As part of the validation of our approach, an independent and double blinded visual inspection of breast sample spectra was also performed as reported in the previous paragraph and confirmed that the samples assigned as outliers indeed displayed poor digestion.

The final cohort of BC samples consisted of 34 sections.

A PCA including all the specimens show a complete overlap between all tissue samples. No combination of components allowed a separation between groups, making it impossible to discriminate between BC subtypes (Figure 25a). Removing the samples with bad digestion led to a partial separation of the pCR (green) and nonpCR (pink) patients in the PCA (Figure 25b).

Here, we demonstrate that FFPE pork fat is a suitable QC to monitor day-to-day technical variability that can be due to variation in sample preparation and/or instrument performance. More importantly, it enables measuring on-tissue trypsin efficiency simultaneously and correspondingly to that of the BC tissue. Furthermore, visually comparing the spectra features by manual scoring was in full compliance to the PCA and pLSDA results.

\section{Prediction of $p C R$ and non-pCR in BC tissues}

Tumor response to therapy differs considerably from patient to patient, ranging from the pathologic complete response (pCR) to non-response (non-PCR). Predicting pCR in breast cancer after neoadjuvant systemic therapy (NST) is still not possible with current imaging routine examination. Predicting the response from biopsy samples taken before NST to determine which patient will benefit from therapy is still challenging.

In the previous paragraph, we demonstrated that the pork fat is an adequate QC to monitor the digestion efficiency for FFPE-BC tissues, and it allows to filter bad 
digested samples from our analysis. Here, we evaluate the relevance of using our QC strategy to improve the separation of pCR and non-PCR groups in our dataset and therefore improve our ability to predict the tumor response after NST.

PCAs and pLSAs were performed at a pixel level in order to visualize differences between the pCR and non-pCR groups of patients. As reported in the previous paragraph, after exclusion of BC samples using the Pork fat, the PCA and pLSDA showed a separation between groups (Figure 25b and Figure $23 c / d)$.

From the volcano plot, several peaks were found to be discriminative between the groups (Figure 26, i.e. $m / z 656.3$ and 1627.4 further ID will be performed).
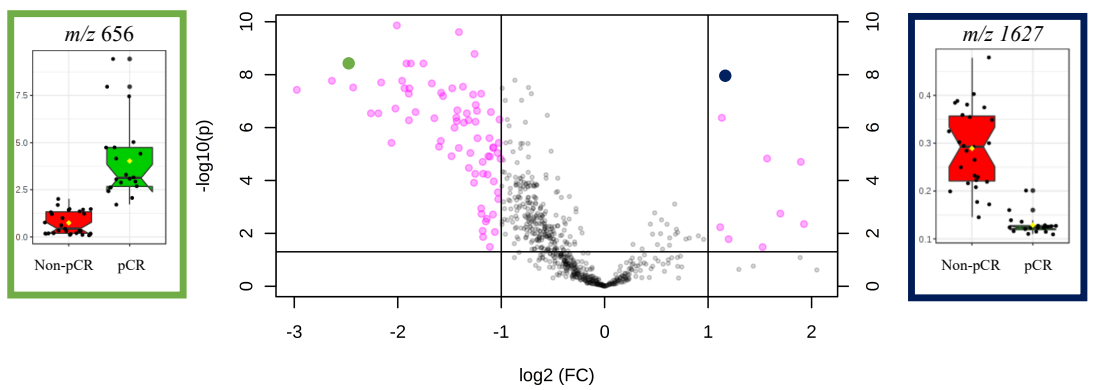

Figure 26 - Volcano plot highlighting feature of importance contributing to the separation between the pCR and nonpCR groups. The fold change threshold was set at 2.0, p-value threshold at 0.05 (FDR-adjusted). Highlighted the m/z values 656.1 and 1627.5 related to pCR and non-pCR, respectively and corresponding box plots.

Finally, $27 \%$ of the tissue samples were used to build a classification model to test the ability to predict pCR and non-pCR. The classification model was built on a pixel level from annotated tumor areas and focuses on tumor area in order to account for tissue heterogeneity (Figure 27). 


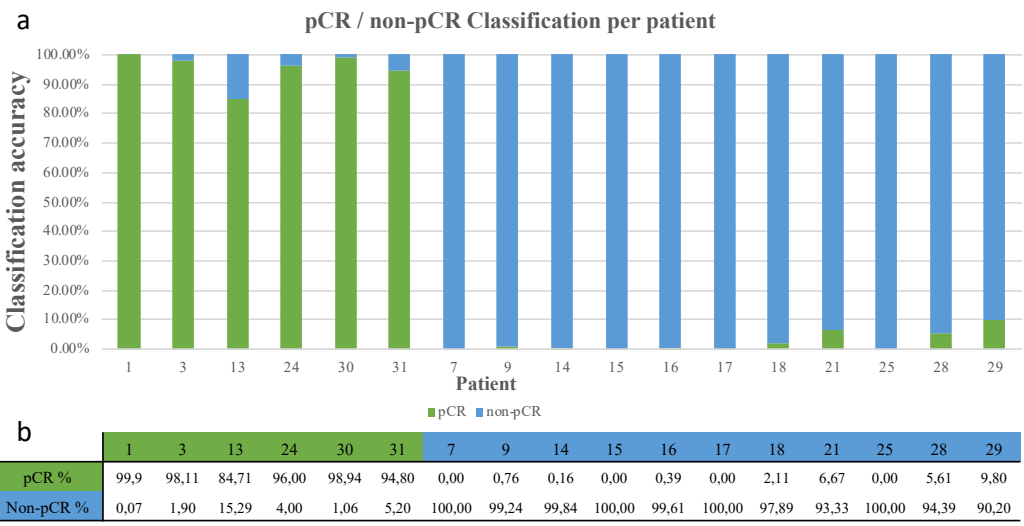

Figure 27 - Result of the supervised multivariate classification model of pCR versus non-pCR BC samples run at a pixel level. (a) On the $y$-axis the classification accuracy indicates the percentage of the variance in the data to be assigned correctly into pCR or non-pCR. The x-axis reports the patient number (supplementary data) and to be pCR or nonpCR patient. (b) Corresponding Table indicates the specific percentage of data per patient to be assigned to pcr or non-por.

The model was then validated with the remaining $73 \%$ of the samples. The validation report displayed a correct classification of pCR tumors within the range of 94.8 - 99.9\%.The non-pCR tumors were correctly classified within the range 90.2100\%. This indicates that the classification model is able to classify pCR and non-pCR tissue with an accuracy in the range 90.2 and 100\% (Figure 28).

This proves that a quality control based on FFPE tissues (pork fat) is a suitable model to account for technical variations in MALDI-MSI experiments and correctly identify outlier samples to be excluded from the data analysis. As a consequence, we prove that this is applicable to a concrete clinical example improving the outcome of the analysis.

\section{Investigation of $p C R$ and non-pCR in BC subgroups}

$\mathrm{BC}$ can also be classified into different subtypes according to well known receptor protein biomarkers. Hormone receptor positive or negative $(\mathrm{HR}+, \mathrm{HR}-$ respectively) The most common hormones receptors tested are the estrogen receptor (ER) and progesterone receptor (PR) which represent the 50-65\% of BCs [153]. Human epidermal growth factor 2, positive (HER2+) represents the 10-20\% of BCs [8]. Lastly, triple negative (ER, PR and HER2 negative) represent the $10-20 \%$ of BCs 
[153]. In the first analysis, all tumors were included, regardless of their HR- and HER2- status. However, the HR- and HER2- status influence the response to treatment. It is well known that receptor status affects the pathologic complete response (pCR), metastatic patterns, relapse and clinical outcome of patients. Patients with triple negative breast cancer (TNBC) are strongly associated with a better long term prognosis if they reach a pCR after treatment as are patients with HER2+ HR- tumors who receive trastuzumab [154].

Therefore, in order to investigate the influence of the receptor status on our analysis and prediction model, annotated tumors were then divided into subgroups based on the clinically relevant HER2 and ER status. PCA analysis of the subgroups indicated a separation of the TNBC subgroup and the ER- HER2subgroup (Figure 28).

\section{PCA BC Tumor areas per subtype}

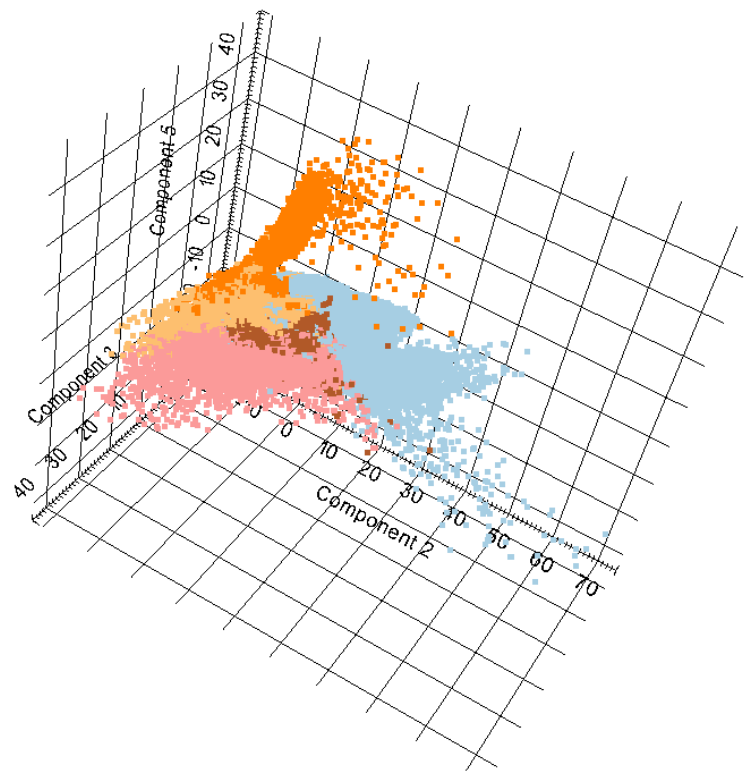

\section{TNBC}

ER+HER2+

ER-HER2+

ER+HER2-

ER-HER2-

Figure 28 - Result of the PCA on BC tumor subtypes. A 3D view represents component 2,3 and 5 as separating factors of the TNBC, ER and HER2 subgroups. 
The other subgroups are overlapping in the PCA result; thus, are not separated.

While PR-status was not taken into account since it is clinically less relevant, it might add additional information or contribute to the response to NST.

The separation of TNBC and ER-HER2- achieved by PCA is a representative result due to its application on a small cohort of patients.

Further investigations with a larger cohort including more patients from each subtype have to be performed on prediction of PCR or non-pCR after NST with more tissues since biological differences are not representative of the heterogenic population of BC tumors. BC tissues are known to be heterogenic: different cell types are observed between individuals with the same tumor type but also in individual tumors and their surroundings. The surroundings of the tumor, and infiltration of nontumor cells can influence response to treatment $[155,156]$. Investigating a larger cohort might also enable to subdivide the BC tumors based on the PR- receptor status. Which was a main limitation of our approach due to the small size of our clinical cohort when it comes to the analysis of subgroups. 


\subsection{Chapter Conclusions}

Enstablishing a reliable QC for MSI analysis is of upmost importance to monitor the sample preparation and analysis of large cohorts of samples.

We proved that pork fat is a suitable QC for FFPE analysis of tissues. In this chapter, we demonstrate how thanks to the pork it was possible to detect and remove outliers from a FFPE - BC tissues analyzed using MSI. Before the data selection, a separation of pCR and non-pCR BC tissues was not possible. After removing the BC tissue outliers, the PCA results indicates that separating pCR from non-pCR is possible. This was also strongly confirmed by the classification model. Upon subdividing the BC tissues, separating pCR from non-pCR was only possible in the TNBC and ER-HER2- subgroup. However, subdividing the BC tissues based on HER2 and ER status led to very small number of BC tissues included per subgroup analysis. There were too little samples to build a classification model or to draw a conclusion from the subgroup analysis. We can only speculate on the preliminary data of our subgroup analysis that separating pCR from non-pCR in BC subgroups is possible. In future experiments we recommend to expand the cohort, enabling further analysis of separating PCR and non-pCR in larger BC subgroups datasets.

The cytochrome $\mathrm{C}$ gave false indications about the spectra and digestion quality in 19 out of 54 measurements (35\%) while the pork fat only gave a wrongful indication in 1 out of 54 measurements (2\%).

From these results we can conclude that pork fat is a promising QC, able to monitor on-tissue trypsin digestion, day-to-day variability and experimental variability of FFPE tissues. 



\section{Chapter 5}

Tissue classification by Rapid Evaporative Ionization Mass spectrometric (REIMS)

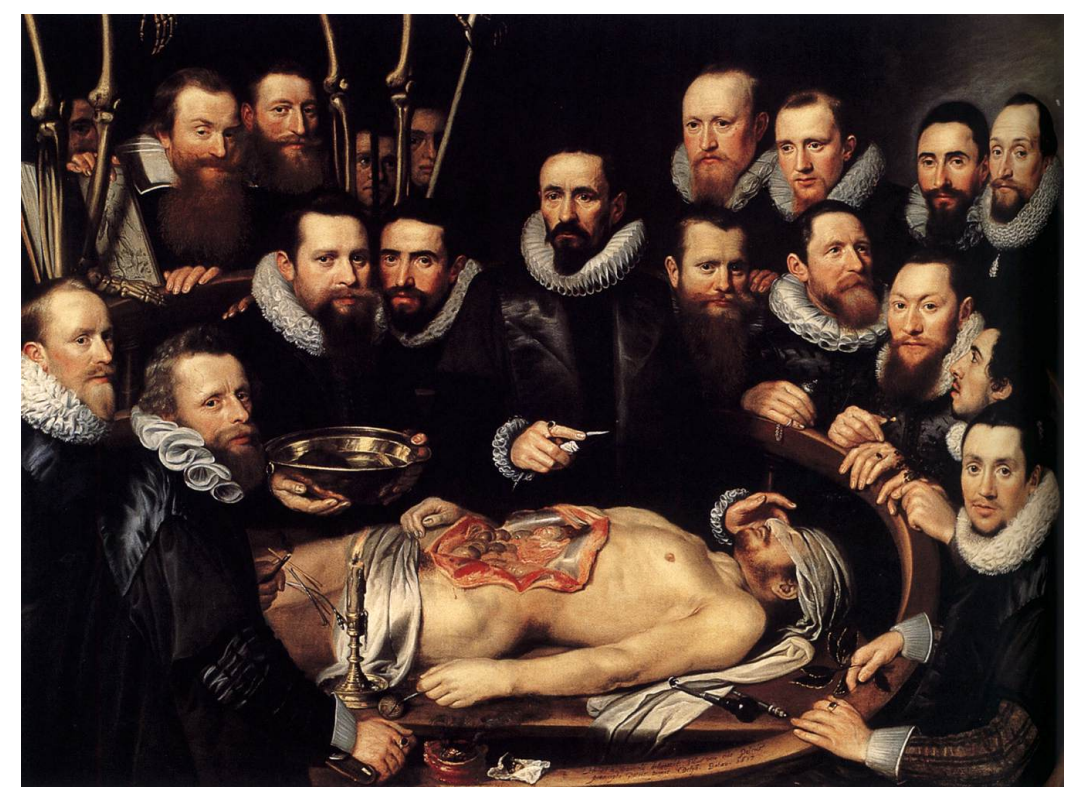

Figure 29 - Michiel van Mierevelt (1566-1641) and Pieter van Mierevelt (1596-1623), The Anatomy Lesson of Dr. Willem van der Meer (1617), oil on canvas, $146.5 \times 202 \mathrm{~cm}$, Museum Prinsenhof Delft, Delft, The Netherlands. Wikimedia Commons

\section{Adapted from:}

Tissue classification by Rapid Evaporative lonization Mass Spectrometric (REIMS): comparison between a diathermic knife and $\mathrm{CO}_{2}$ laser sampling on classification performance

Michele Genangeli, Ron M.A. Heeren, Tiffany Porta Siegel

Analytical and bioanalytical chemistry, Accepted August 2019 


\section{$5 \quad$ Tissue classification by Rapid Evaporative lonization Mass Spectrometry (REIMS): comparison between a diathermic knife and $\mathrm{CO}_{2}$ laser sampling on classification performance}

\subsection{Abstract}

The increasing need for rapid, in situ and robust tissue profiling approaches in the context of intraoperative diagnostics has led to the development of a large number of ambient ionization-based surface sampling strategies. This paper compares the performances of a diathermic knife and a $\mathrm{CO}_{2}$ laser handpiece, both clinically approved, coupled to a rapid evaporative ionization mass spectrometry (REIMS) source for quasi-instantanous tissue classification. Severel fresh meat samples (muscle, liver, bone, bone marrow, cartilage, skin, fat) were obtained from different animals. Overall, the laser produced cleaner cuts, more reproducible and higher spectral quality signals when compared to the diathermic knife (CV laser $=9-12 \%$, CV diathermic $=14-$ 23\%). The molecular profiles were subsequently entered into a database and PCA/LDA classification/prediction models were built to assess if the data generated with one sampling modality can be employed to classify the data generated with the other handpiece. We demonstrate that the correct classification rate of the models increases $(+25 \%)$ with the introduction of a model based on peak lists that are tissue-specific and common to the two handpieces, compared to considering solely the whole molecular profile. This renders possible to use a unique and universal database for quasiinstantaneous tissue recognition which would provide similar classification results independent from the handpiece used. Furtermore, the laser was able to generate aerosols rich in lipids from hard tissues such as bone, bone marrow and cartilage. Combined, these results demonstrate that REIMS is a valuable and versatile tool for instantaneous identification/classification of hard tissue and coupled to different aerosol generating handpieces expands its field of application. 


\subsection{Introduction}

Accurate tumor diagnosis during surgery solely relies on histopathological evaluation of frozen section and is still challenging. Surgeons would benefit from instantaneous feedback during surgery to improve tumor resection and ultimately patient outcome. The need for more precise oncological surgical procedures depends on the availability of rapid, in situand robust molecular approaches. This has led to the development of a large number of ambient ionization mass spectrometry (MS)-based surface sampling strategies [157-160]. Amongst these ambient ionization techniques, desorption electrospray ionization (DESI), or laser ablation electrospray ionization (LAESI), to name a few, have been developed as robust solutions for lipidomic/metabolomic imaging and profiling $[161,162]$. However, they are not suited for in-situ rapid analysis of "fresh" or intact samples. Other sampling techniques such as the "MasSpec Pen" [163], "Spidermass" [164, 165], cavitron ultrasonic surgical aspiration/sonic spray ionization (CUSA/SSI) [22], have been hyphenated to ambient ionization MS to enable rapid intraoperative diagnostics of human cancers. The MassSpec Pen makes use of a simple and disposable hand-device which allows for non tissue-distructive sampling. However, Saudemont et al. recently reported that the MassSpec Pen could have limited use for in vivo cancer diagnostics due to possible contamination of the system with tissue debris, which is caused by the fact that the device has to be operated in contact to the tissue [166]. The "Spidermass"-approach is based on a modified optical parametric oscillator (OPO) system pumped by an Nd:YAG infrared laser to superficially ablate tissue (micro invasiveness) for in vivo tissue classification [165]. Clinical implementation of these two techniques for intraoperative diagnostics remains limited because they have not yet been approved as a medical device. Most of the beforementioned techniques allow only the sampling of specimens under ambient conditions, and cannot be used to resect tissues. Woolman et.a/reported the combination of DESI-MS and a picosecond infrared laser (PIRL) MS as an ablation probe requiring minimal tissue removal for ex-vivolin-situ imaging operations $[167,168]$. They also suggested how the integration of PIRL with rapid evaporative ionization mass spectrometry (REIMS) is likely to increase the 
robustness of signal and reproducibility of PIRL due to the aspiration driven by the Venturi pump [169].

REIMS is an ambient ionization technique which involves the aspiration and rapid thermal ionization of aerosols produced from electrocautery, ultrasonic aspiration [22], ultraviolet (UV) and infrared (IR) lasers [23-26]. The most common handpieces employed to generate smoke and combined with a REIMS system are monopolar and bipolar electrocautery/diathermic (blade/needle) knives routinely used in the OR for surgical procedures [24]. A monopolar device uses a higher voltage when compared to a bipolar device and has a better ability to cut and coagulate large bleeding areas. Thus, a monopolar accessory is mainly used to cut and coagulate relatively large tissues while the bipolar electrocautery is more used for smaller tissue applications (e.g. brain surgery) or for the classification/identification of bacteria and fungi $[27,28]$.

Amongst other certified medical devices, a $\mathrm{CO}_{2}$ laser [21] can also be used intraoperatively due to: i) its infrared wavelength of $10.6 \mu \mathrm{m}$ highly absorbed in water molecules contained in tissue [30-32]; and ii) its ability to produce both a high-power continuous wave (i.e. for cutting) and pulsed and super pulsed waves (i.e. for sampling $[164,169])$. The strength of the approaches described above are the use of standard surgical devices that are already frequently employed in a surgical setting. No modifications of the surgical procedures are needed while surgeons are offered an opportunity to make better informed decisions. This facilitates the easier take up and acceptance by the medical community.

REIMS was originally developed for oncological surgical applications [24, $33,34]$ with the ultimate goal to improve patient outcome after surgery by ensuring the removal of all malignant tissue. The applicability of REIMS for cancerous tissue diagnostics has been demonstrated both ex vivo and in vivo in the operating room with instantaneous tissue classification ("normal"/surrounding tissue vs. tumor or tumor margins). The ex vivo 
procedure entails a surgical ressection of biological material, followed rapildy by a REIMS enabled assesment of the tissue outside the patient, while the in vivo studies are conducted during ressection of the material from the patient.

REIMS has also been used for food analysis (e.g. to identify the species of origin of meat products) [170] and for identification of bacterial colonies and direct in vivo mapping of bacterial growth $[171,172]$. The combination of REIMS with multivariate analysis of the data collected from the aerosols allows for classification of the dissected tissues within seconds [172]. Different statistical methods are employed to build a tissue recognition model based on tissue-specific molecular profiles. From the literature, principal component and linear discriminant analysis (PCA/LDA) are mainly reported for this purpose $[24,33,34,160,172-174]$. The entire molecular profile within the mass range $\mathrm{m} / \mathrm{z} 600-900$ - which corresponds to the range of detection of glycerophospholipids - is used to build these models. Differences in the relative intensity of lipid species are included to maximize the variance between two or more groups. Lately, Cordero Hernandez et al. reported that the classification rate of tissues can be improved by selecting tissue-specific peaks and generating new models based on both PCA/LDA of acquired molecular profiles [175].

Here, we report the benfits and limitations of two surgical handpieces: a $\mathrm{CO}_{2}$ laser and a diathermic knife to generate smoke from fresh animal tissues. These handpieces were coupled to a REIMS-quadrupole-time of flight (qTOF) systems for quasi-instantanous tissue classification. For this purpose, fresh meat samples (muscle, liver, bone, bone marrow, cartilage, skin, fat) were obtained from different animals and analyzed using the two sampling tools. Generated aerosols were directly analyzed via REIMS and tissue/species-specific molecular profiles were recorded. The handpieces were compared in terms of tissue damage/invasiveness, spectral quality and signal-to-noise ratio. The molecular profiles were subsequently entered into a database and classification/prediction models based on PCA/LDA were built. The goal of our next investigations was to demonstrate whether it was possible to build a unique and universal database for quasi-instantaneous tissue recognition which 
would provide similar classification results independent from the handpiece used. Therefore, we compared the tissue classification rates and reproducibility of: i) the PCA/LDA molecular classification models built first with data generated with one sampling modality and then employed to classify the data generated with the other handpiece; ii) a more targeted approach where the models were built based on features that are common for both handpieces. The general workflow is reported in Figure 30 . We confirmed that the classification rate of the models increases with the introduction of a model based peak lists that are tissue-specific; which renders it possible to use one database for both handpieces. Finally, we demonstrate that the $\mathrm{CO}_{2}$ laser can be used as a sampling tool for hard tissues such as bone, cartilage and bone marrow, expanding the field of applications and the versatility of REIMS for tissue classification.

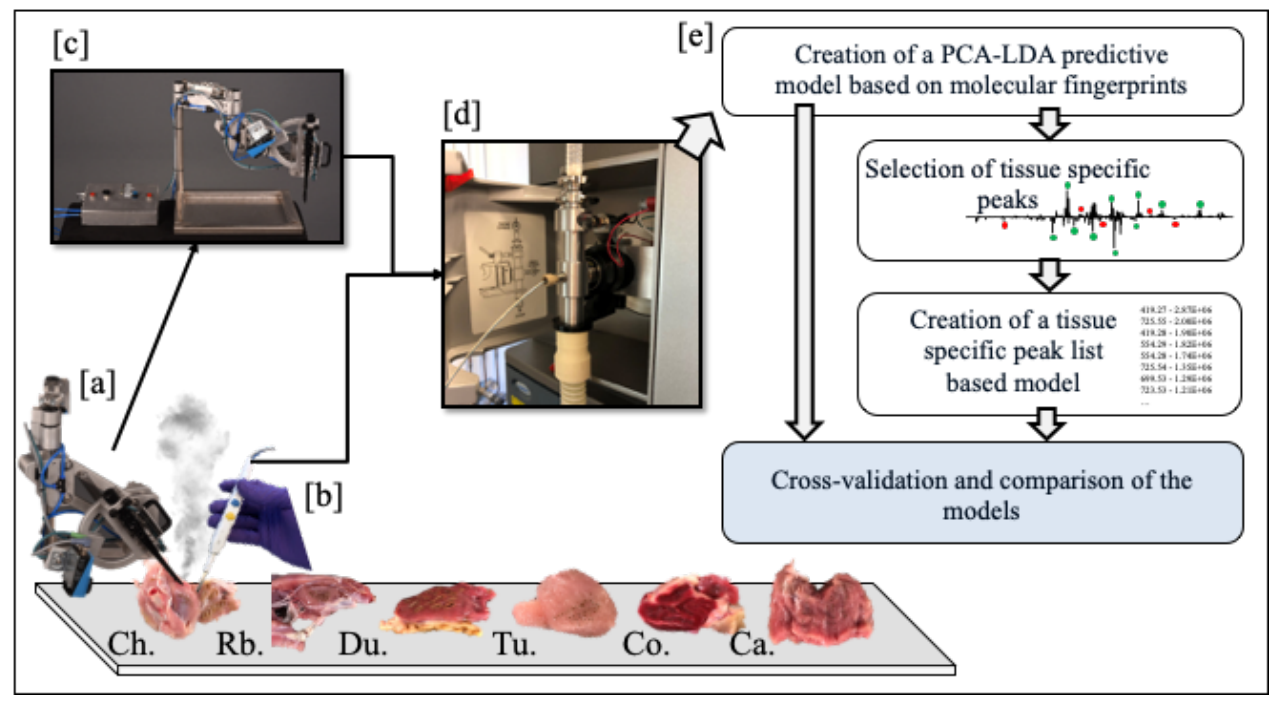

Figure 30 - Experimental workflow from tissue sampling to the creation of tissue classification models: Every tissue (Chicken [Ch.], Rabbit [Rb.], Duck [Du.], Turkey [Tu.], Cow [Co.] and Calf [Ca.]) was sampled and analyzed with both the CO laser [a] and the electrocautery knife [b]. For safety reasons, the laser was mounted on a home-built mechanic arm [c] maintained in a fixed position during laser ablation. Both handpieces were connected to the REMS source and the data collected from vapors were acquired in negative ionization mode [d]. After basic data preprocessing, several PCA-LDA predictive models were created based of the overall molecular (lipids) fingerprint [e]. From the loading plots of each model, tissue-specific peaks were selected to create peak lists common to the two handpieces. This peak list was further used to build a more targeted model to improve the classification rate of our statistical models that are now able to classify tissues independently from the handpiece used for sampling. 


\subsection{Materials and methods \\ 5.3.1 Materials and reagents}

Isopropyl alcohol, ethanol, methanol, xylene and water (ULC/MS-CC/SFC grade) were purchased from Biosolve Chimie (Dieuze, France). Norhamane was purchased from Merck KGaA (Darmstadt, Germany). Leucine-enkephalin was purchased from Sigma-Aldrich (St. Louis, US).

\subsubsection{Diathermic knife}

Electrosurgical dissection was carried out using a commercial monopolar electrosurgical unit COVIDIEN Force Fx electrosurgical generator (COVIDIEN Ltd., Ireland) providing power-controlled sinusoidal $330 \mathrm{kHz}$ alternating current and a custom handpiece and active -cutting- electrode (Blade length $=1.7 \mathrm{~cm}$, width $=0.2$ cm, thickness $=0.05 \mathrm{~cm}$ ) modified by Waters (Manchester, UK) with a smoke evacuating line (tubing system). The generator was used in "cut" mode with a power setting range of 20 to $30 \mathrm{~W}$. All the ex vivo samples were placed on a return electrode of the electrosurgical setup. The heater in the REIMS source was kept at $300^{\circ} \mathrm{C}$. In order to maximize reproducibility, the burns made with the diathermic knife were carried out while keeping the knife vertical to the tissue being analyzed, and the signal coming from the smoke was recorded for 3 seconds.

\subsubsection{Surgical $\mathrm{CO}_{2}$ laser}

All experiments were performed with an AcuPulse Class IV $\mathrm{CO}_{2} 10.6 \mu \mathrm{m}$ surgical laser unit (Lumenis GmbH, Germany) with a maximum power of 60W. The laser can be operated in three distinct modalities (continous wave [CW], pulsed [PW] and super pulse mode [SPW]). Continous wave mode produces a low peak power, it has a superficial 'soft' impact and a wider thermal zone (spot size 1.3 mm, $170 \mathrm{~mJ}$ energy, 60\% density and 150-200 $\mu \mathrm{m}$ penetration depth (Figure 32). The pulsed and super pulse modalities have a high and very high peak power respectively, a deeper impact and a narrow and controlled thermal zone (spot size $0.12 \mathrm{~mm}, 20 \mathrm{~mJ}$ energy, 15\% density and 700 um penetration depth; Figure 32) [176]. The laser was used in "cut" 
pulsed mode with a laser power ranging between 18-20 W (matched to the tissue being analyzed), using single pulse time of $0.15 \mathrm{~s}$. For hard tissue analysis only, a higher power was necessary in pulsed mode to generate sufficient ions due to the relative low water content of the bone and cartilage. The laser was fixed to a home-built mechanic arm (Figure 31) for safety considerations. This consequently increased the reproducibility by keeping the laser at a fixed distance from the samples (focus point).
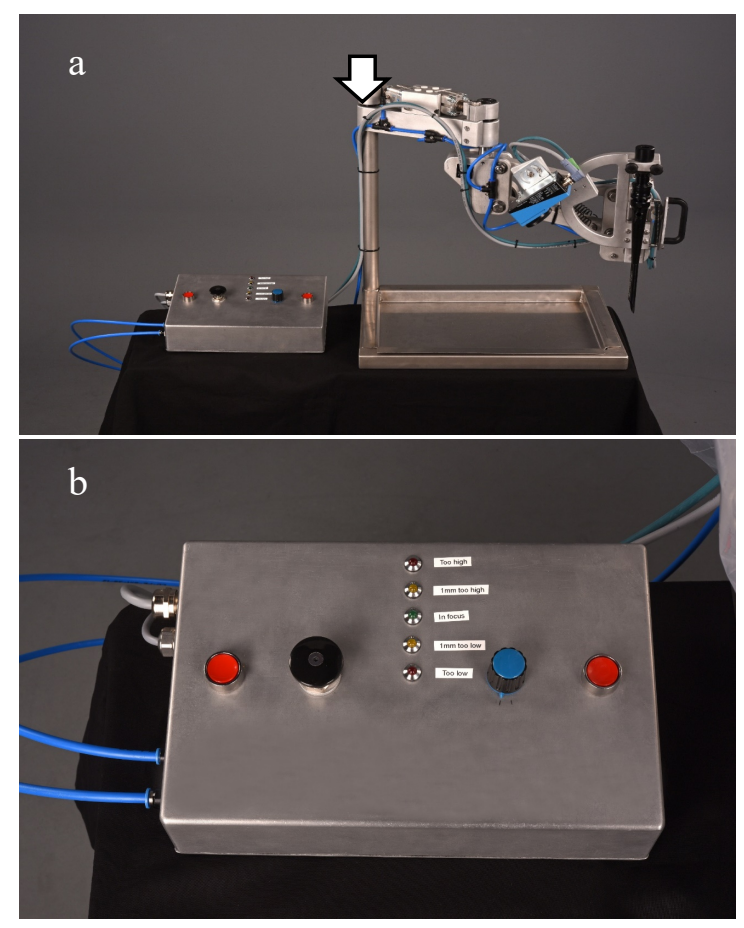

Figure 31 - Home built laser arm used to maintain the laser in a fixed and safe position during analysis [a]. Control box for the safety arm includes height control to ensure that the laser is at optimal focus position on the tissue analyzed[b].

The arm is made of aluminum grade 6082 (chemicals resistant, noncorrosive and light material) and glass bead blasted on the outside (in order to reduce the reflectivity of the aluminum). Each moving part of the arm is kept steady by air powered breaks. A visible-infrared camera (sick inspector PIM60) was mounted next to the laser handle in order to control the laser height from the tissue, keeping it at a fixed distance from the tissue, increasing the 
reproducibility of the data generated. The focus point was controlled by a home-made program which allows the laser to operate only when it is focused on the tissue.

\subsubsection{REIMS - qTOF instrumentation}

The aerosols generated with the two handpieces were analyzed via REIMS. The smoke produced was aspirated by a Venturi pump connected to the REIMS source. The REIMS source was mounted on a qTOF mass spectrometer (G2-XS Waters. Manchester, UK) operated in negative ionization mode. Data was acquired within the mass-to-charge range m/z of 100-1500 and in "sensitivity" mode, at a mass resolution of 15.000 full width at half-maximum (FWHM) at $\mathrm{m} / z$ 600. The instrument was calibrated daily with a solution of sodium formate. A solution of leucine-encephalin dissolved in isopropyl alcohol (1 ng/ $\mu$ l) was continuously infused in the REIMS source at a flow rate of $150 \mu \mathrm{l} / \mathrm{min}$. The deprotonated ion of Leu-Enk was used for external lock mass calibration of the data acquired ( $\left.m / 2554.2615[\mathrm{M}-\mathrm{H}]^{-}\right)$.

\subsubsection{Experimental design}

Meat samples (muscle, liver, bone, bone marrow, cartilage, and skin) from different animals (cow, calf, chicken, turkey, duck, and rabbit) were obtained from a local butcher and analyzed consecutively and on the same day with the surgical laser and diathermic knife (Table 20). An average of $n=20$ burns were recorded for every tissue with both handpieces (with the exception for bone and cartilage as noted in Table 20). The samples were frozen in liquid nitrogen after analysis with both handpieces and then sliced (12 $\mu \mathrm{m}$ thick) with a cryo-microtome (Leica CM 1860 UV, Leica biosystems, Wetzlar, GE); mounted onto histological glass slides (VWR, Leuven, BE) for additional analysis with matrix-assisted laser desorption/ionization mass spectrometry imaging (MALDI-MSI). 
Table 20 - Number of burns collected for each tissue type and for each handpiece. Bone, bone marrow and cartilage were analyzed only with the $\mathrm{CO}_{2}$-laser.

\begin{tabular}{llcc}
\hline Animal & Subtype & n burns Laser & n burns Diathermic \\
\hline Chicken & Muscle & 25 & 25 \\
& Skin & 28 & 20 \\
& Liver & 25 & 22 \\
& Bone & 30 & - \\
& Cartilage & 23 & - \\
Rabbit & Bone Marrow & 9 & 20 \\
Duck & Muscle & & 25 \\
& Muscle & 25 & 15 \\
Turkey & Skin & 25 & 20 \\
Cow & Muscle & 15 & 25 \\
Calf & Muscle & 20 & 25 \\
\hline
\end{tabular}

\subsubsection{MALDI-MSI experiments and lipid identification}

The identification of selected lipid ion species was performed by both MS/MS fragmentation on the REIMS-qTOF and the exact mass measurement from MALDI-MSI on a Q-Exactive Orbitrap (Thermo Fisher Scientific, Massachusetts, US). For the MALDI-MSI experiments, the matrix was sublimed on top of the sample as follows: a solution of $60 \mathrm{mg}$ of norhamane and methanol (2ml) was applied onto the slides with a sublimator device (HTX Technologies, LLC, USA [177]). The REIMS-TOF was set to MS/MS negative ionization mode with variable collision energy (see details in the supplementary materials). For the Orbitrap, the laser power was set to $1.6 \mathrm{\mu J}$ per pulse at $1 \mathrm{kHz}$, the resolution was set at 240.000 in negative ionization mode at m/z200-2000. Alex123 and LIPID metabolite and Pathways Strategy Lipidomic Gateway (LIPIDMAPS ${ }^{\circledR}$ ) were used for the lipid identification based on the exact mass and MS/MS data. The identification of selected peaks of interest was performed limiting the search to only deprotonated glycerophospholipid and fatty acid ions in the $\mathrm{m} / \mathrm{z}$ 
range 600 - 900 with a mass tolerance of +/- 0.1 Da for the REIMS and +/- 0.002 Da for the Orbitrap (Table A2-A6).

\subsubsection{Hematoxylin and Eosin histological staining (H\&E)}

The H\&E staining protocol used consisted of: i) matrix removal from the plates with a $100 \%$ ethanol (2x) bath for 3 minutes; ii) rehydration in subsequent ethanol baths of 96\% (2x) and 70\% (2x), each for 2 min; iii) staining with hematoxylin for 3 min, washed in a deionized water bath; iv) staining with eosin for $30 \mathrm{sec}$; v) final wash in water (3 min), ethanol (1 min) and xylene (30 s). Once dry, the slides were covered with a coverslip that was fixed with Entellan (Merk KGaA, Darmstadt, Germany). Highresolution optical images of the H\&E stained slides were acquired with a MIRAX Desk Scanner (Zeiss, Jesa, Germany).

\subsubsection{Multivariate data analysis and building of the statistical models}

Mass spectral processing and multivariate data analysis were performed using the Abstract Model Builder (AMX) software ([beta] version 1.0.1581.0, Waters Research Center, Budapest, Hungary). The mass spectra were background subtracted; mass drift corrected based on the reference peak at m/z554.2615 [M-H] ${ }^{-}$corresponding to the deprotonated ion of Leucine-Enkephalin used as lock mass. Peaks were binned to 0.1 Da within the mass range $\mathrm{m} / 2$ 600-900. The spectra were all normalized against the total ion count (TIC). The resulting data was used to create two classification models: i) a principal component analysis/linear discriminant analysis (PCA/LDA) model based only on molecular fingerprints which is used to identify tissue-specific peaks; and ii) a peak list-based PCA/LDA model. PCA was performed with a maximum of 10 dimensions and LDA with $n-1$ dimensions where $n$ is the number of tissue types introduced in the model. Several PCA considering increasing numbers of components were tested and the results showed that with using a maximum of 10 components we preserve $>98.8 \%$ of the total variance of the data. A series of internal cross-validation tests were performed by building the classification models from $80 \%$ of the data and 
validating it with the remaining $20 \%$, or by validating a model generated with one sampling modality, such as the laser, with the data generated with the other modality, such as the diathermic knife, and vice versa. For all cross-validation, outliers were identified based on standard deviation (sd) as follow: when a feature deviates $5 \mathrm{sd}$; it is considered as an outlier. The TIC of twenty consecutive burns ( $n=10$ for the diathermic knife and $n=10$ for the laser) from every tissue type were compared and the results expressed in terms of coefficient of variation (CV). The average signal-to-noise ratio (S/N) from ten selected peaks over five consecutive burns, was calculated and the average results were reported in terms of $\mathrm{CV}$.

\subsubsection{Safety considerations}

All samples were analyzed in a class II biosafety cabinet. All solvents used for the analysis and cleaning were handled according to the material safety data sheet provided by their respective manufacturer. In order to comply with the safety regulations of using a $\mathrm{CO}_{2}$ laser in our laboratory and increase the reproducibility of the data generated with the laser, a dedicated mechanic arm allowing the laser to be operated at fixed position was developed (see the "Surgical Laser" section). The laser was fired through a two-button safety box placed outside the biosafety cabinet (Figure 31-b), while wearing $\mathrm{CO}_{2}$ laser grade safety goggles in a reserved area shielded with class IV safety curtains.

\subsection{Results}

\subsubsection{Visual assessment of tissue damages and evaluation of spectral quality for real-time analysis of soft tissues}

Here, we compare the main differences between the two handpieces (laser and diathermic knife) through a comparison of: i) tissue damage and handpiece characteristics; ii) stability and reproducibility of the generated signals; and iii) qualitative assesment of generated mass spectra. The same tissue samples were consecutively analyzed with the diathermic knife and laser 
in order to facilitate a direct comparison of the handpieces and limit instrumental variability.

\section{Comparison of handpiece's characteristics and tissue damage.}

The tissue damage caused by two handpieces were macro- and microscopically examined. Figure 32a/c shows a macroscopic image of cow liver samples burnt with the laser and diathermic knife. It clearly demonstrates that tissue damage caused by the laser is more confined and localized than damage cause by the diathermic knife. This is mainly due to the width of the laser beam which is smaller than the blade used with the diathermic knife. In order to confirm and illustrate these observations, the same tissue was frozen in liquid nitrogen, cut with a cryotome and H\&E stained. As shown in Figure 32b/d, the laser produced tissue burns of $\approx 0.39 \mathrm{~mm}$ radius while the diathermic knife (equipped with a blade-electrode) produced much bigger burns of $\approx$ widht $=0.79 \mathrm{~mm}$, length $3.65 \mathrm{~mm}$. It was hypotesized that the minimal material ablated, and thus the reduced smoke generated by the laser, led to more precise sampling. 


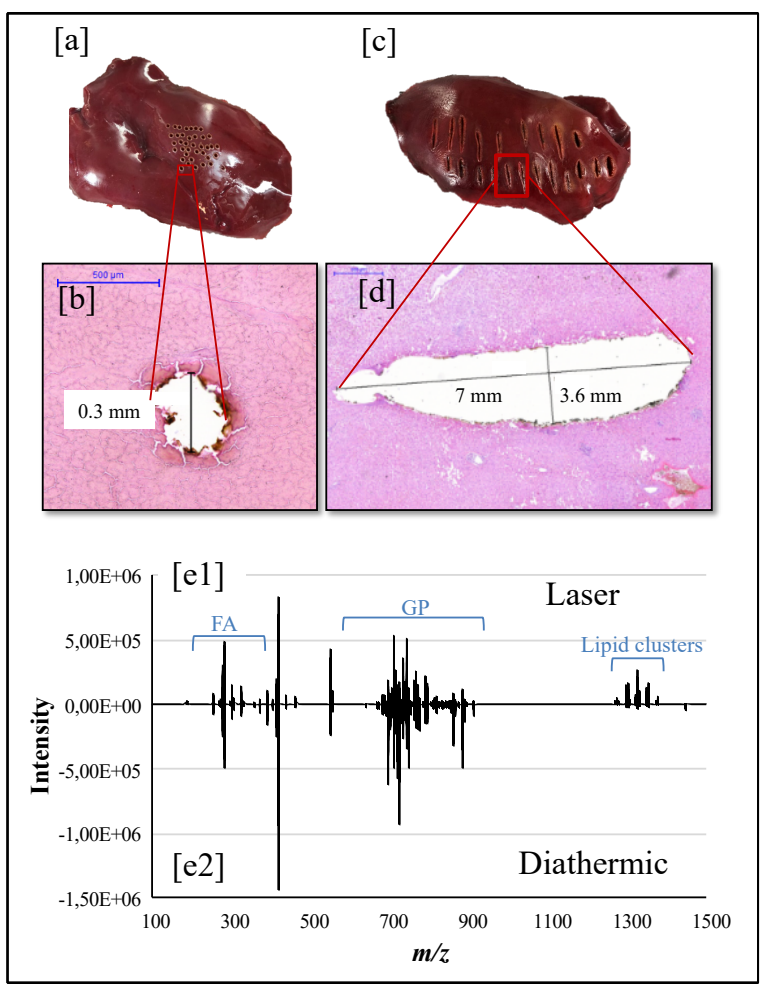

Figure 32 - Macroscopic [a,c] and Microscopic (H\&E stained) [b, d] visualization of the tissue damages generated after laser $[a, b]$ and diathermic samplings $[c, d]$. The laser generated a smaller tissue damage compared to the diathermic knife (laser $\varnothing=0.3 \mathrm{~mm}$, Diathermic $\varnothing=7 \mathrm{~mm}$ wide $/ 36 \mathrm{~mm} / \mathrm{ong}$ ). The laser also produced a more reproducible signal, reduced spectral noise and more lijpid clusters (in the region $\mathrm{m} / \mathrm{z} 1200-1500$ ) [e1-2].

\section{Signal stability and reproducibility.}

The laser allowed the generation of lipid-rich smoke with either the continuous wave, pulsed or super pulse modes (Figure 33) for either cutting or surface sampling, demonstrating its versatility. We obtained the best results in terms of overall signal intensity with a laser power of $18 \mathrm{~W}$ and $0.15 \mathrm{~s}$ burn time in pulsed mode for all the soft tissues. 

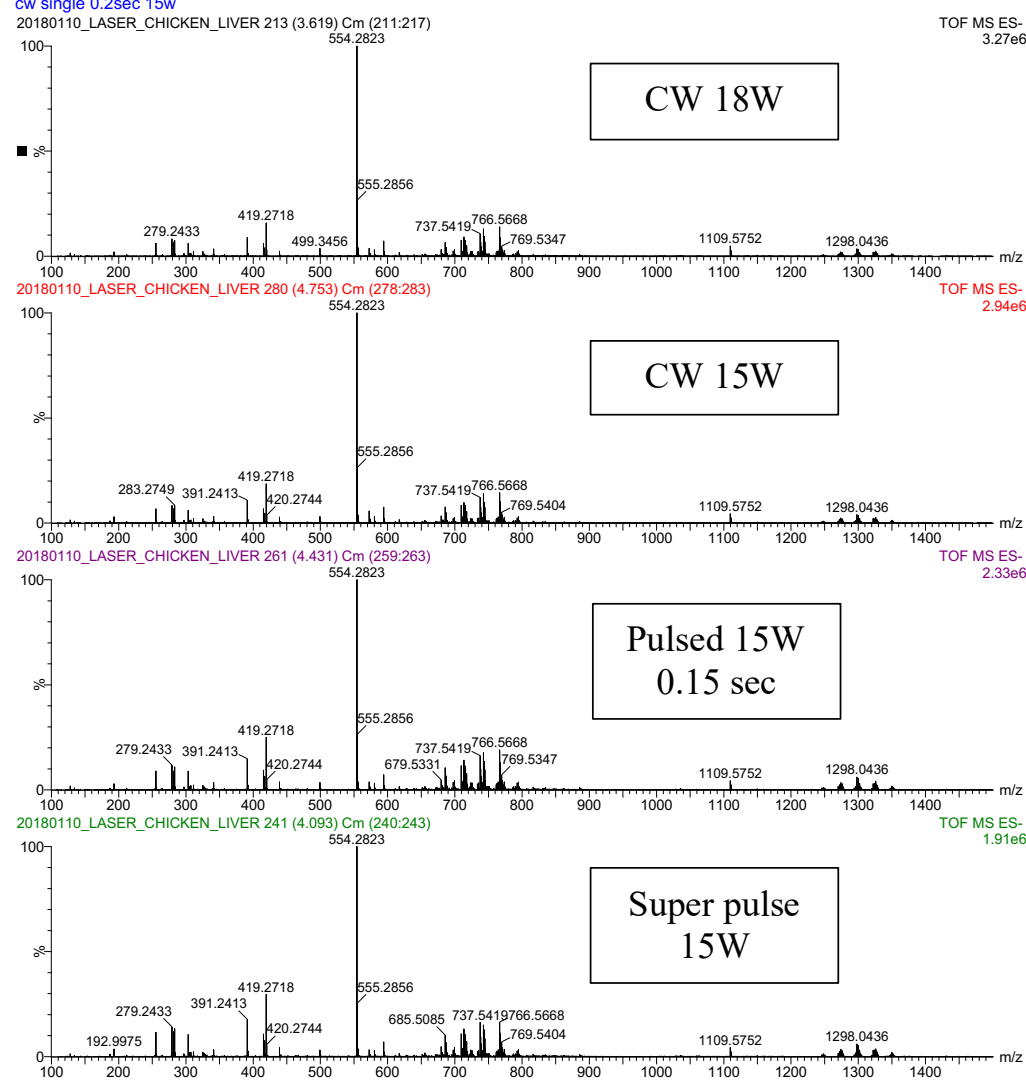

Figure 33 - Laser-REMS spectra obtained from sampling chicken liver with different modalities. CW = continuous wave, Pulsed and super pulse modalities)

The diathermic knife generated a more intense signal from the lipid species with a power of $25 \mathrm{~W}$ in cut mode. We observed the following: the possibility to control the penetration power, wave type and position of the laser in respect to the sample being analyzed led to cleaner spectra and more intense signal intensities overall compared to the diathermic knife. The total ion count (TIC) of $n=10$ mass spectra (From muscle, from all animals, 10 for the laser and 10 for the diathermic) from consecutive burns revealed a higher burn-to-burn reproducibility for the laser with a coefficient of varation (CV) ranging between 9.76-12.00\%. The CV of the signal measured with the diathermic knife for different burns was between 13.92-22.72\% for different tissues. The background noise level was constant for all tissues when analyzed with the same handpiece. The diathermic knife showed a noise level 1.6 
times higher than with the laser. The S/N ratio for the laser was (calculated from 10 peaks of the same tissue analyzed with both the handpieces) $\approx+17$ times higher than for the diathermic knife (see Table 21).

Table 21 - Results from the spectra comparison between the laser and diathermic knife.

\begin{tabular}{|c|c|c|c|c|c|}
\hline & $\mathrm{TIC}$ & Noise level & $\mathrm{S} / \mathrm{N}$ & $\begin{array}{c}\text { n peaks } \\
\text { detectable }\end{array}$ & $\begin{array}{c}\text { Avg. intensity } \\
n=10 \text { peaks }\end{array}$ \\
\hline & $(n=10), C V$ & $n=10$ & $s / 3 n,(n=10)$ & & $\begin{array}{c}\mathrm{n}=5 \text { consecutive } \\
\text { burns, } C V\end{array}$ \\
\hline Laser & $11,31 \%$ & - & $+1,7 x$ & $+7 \% *$ & $0,76 \%$ \\
\hline Diathermic & $15,25 \%$ & $1,6 x$ & - & & $1,94 \%$ \\
\hline
\end{tabular}

The laser generated 7\% more peaks than the diathermic knife (see next paragraph), and the intensity of 10 selected peaks through 5 consecutive burns in the same tissue were evaluated resulting in an average peak intensity CV of $0.76 \%$ for the laser and $1.94 \%$ for the diathermic knife (see Table 21).

As previously mentioned, it is worth nothing that during our laser experiments, the laser was kept in a fix position for safety consideration (see Safety paragraph in the Material and Method part) and operated using a fixed laser power and exposure time while the burns with the diathermic knife were manually performed (the burn depth and time were manually evaluated trying to keep a 3 sec burn time and constant handpiece movement through the tissue). The higher reproducibility and the more stable TIC intensity from the laser when compared to the diathermic knife were mainly due to the fixed position and operation parameters of the laser, which allowed more controlled sampling. This statement is supported by a recent publication from Bodai et al. where it is reported that the geometry and position of the electrode also plays a major role in the classification performances of predictive models when using REIMS [178]. 


\section{Qualitative assessment of generated mass spectra.}

The laser generated a higher peak intensity at m/z 1200-1400 and lower intensity in the "fatty acids" (m/z 100-350) and glycerophospholipids/triglycerides (m/z 600-1100) regions of the spectra when compared to the diathermic knife (Figure 32).

MS/MS was performed to identify selected ion species in the mass range $\mathrm{m} / \mathrm{z}$ 600-1100 and the peaks within m/z1200-1400 using both the REIMS-qTOF equipped with the diathermic knife and the MALDI-Orbitrap. Selected peaks were isolated with an isolation window of $\pm 1 \mathrm{~m} / \mathrm{z}$ and fragmented with the REIMS-qTOF. A high collision energy was required (>40 eV) for the peaks in the high molecular weight range of the spectra (m/z 1200-1400). MS/MS for this last group of molecules revealed several glycerophospholipids in the range $\mathrm{m} / 2600-800$ plus a series of fatty acids in the range m/z100-350 (Figure 34). After the REIMS analysis, the same sample was frozen, cut into $10 \mu \mathrm{m}$-thick sections and analyzed with the MALDI-Orbitrap where high mass accuracy measurements were performed for identification of the precursor ions. No species in the range $m / 21200-1400$ were detected in the MALDI-Orbitrap MS spectra (Figure 34). 


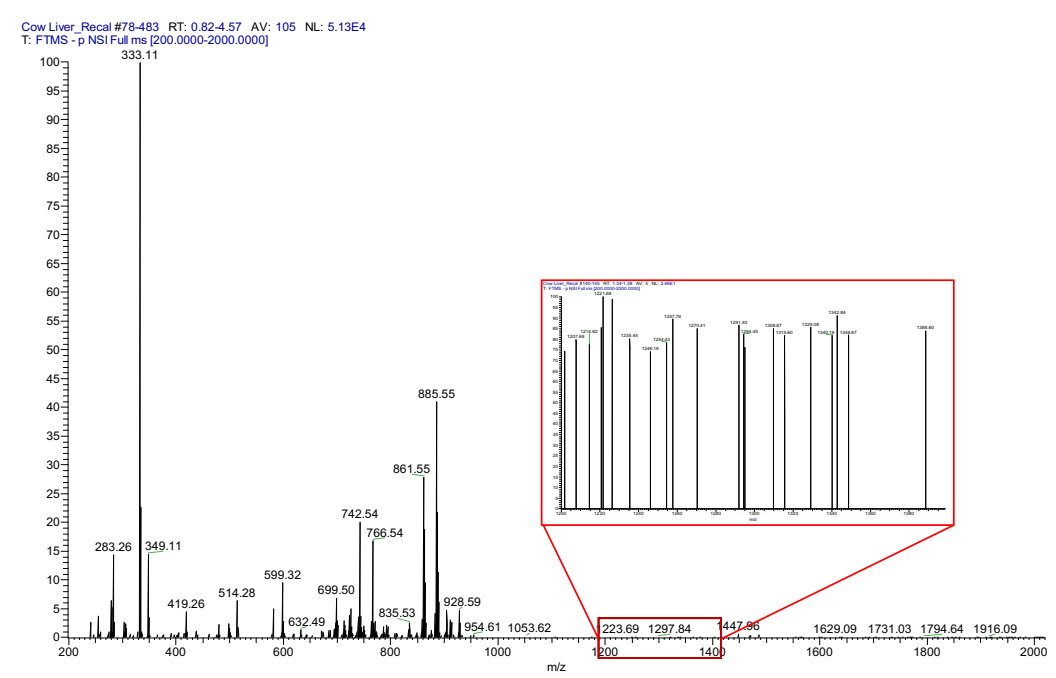

Figure 34 - Mass spectra obtained from a cow liver sampled using the MALDI-Orbitrap and zoom in the region between $\mathrm{m} / \mathrm{z}$ 1200-1400. No clusters are visible in the higher mass range.

The high fragmentation energy required (i.e. $40 \mathrm{eV}$ ) and several lipid species visible in the fragmentation spectra during MS/MS suggest that the species detected with the range at $m / 21200-1400$ are in fact lipid clusters. The lipid clusters were not visible when using MALDI, while they were present when using the $\mathrm{CO}_{2}$ laser (with a high intensity) and diathermic knife (lower intensity). The thermal ablation induced by the $\mathrm{CO}_{2}$ laser and diathermic knife caused the desorption of tissue aerosols that are more prone to the formation of clusters under ambient conditions. The absence of a matrix and ambient pressure (REIMS) also contributed to the formation of lipid clusters [179-181]. Furthermore, the high energy released from the diathermic knife caused either a higher fragmentation or reduced cluster formation during sampling in comparison to the laser [182-184]. A further discussion of the formation and behavior of the lipid clusters is beyond the scope of this study.

The laser overall generates a better $\mathrm{S} / \mathrm{N}$ and a more stable and reproducible signal when compared with the diathermic knife. It is worth remembering that the laser was operated in a fixed position and the beforementioned results are partly due to the usage of a safety arm. It was not 
possible to mount the diathermic knife onto the same robotic arm because, at its current state, the arm cannot raster or be programmed for electrocautery applications. On the other hand, the laser promotes the formation of lipid clusters resulting in a lower intensity for species of interest such as glycerophospholipids (but yet higher S/N with respect to the same species analyzed with the diathermic knife due to reduced background signal).

\subsubsection{Development of universal and more targeted tissue classification}

Our ultimate goal is to improve the overall performance of our classification models (i.e. accuracy, robustness and classification rate) and develop a unique database to correctly identify tissues independetly from the handpiece used for sampling. Several classification models were generated for this purpose with the data acquired with the $\mathrm{CO}_{2}$ laser and the diathermic knife independently. Subsequently, one model was applied to the other data set to assess versatility of the classification model. The first classification models generated were based on molecular patterns solely, which helped to identify specific features from tissue types and common to the two handpieces to build more targeted classification models. The cross-validations were performed by keeping the value for the standard deviation multiplier for the outliers equal to 5.

\section{Classification models based on molecular fingerprints}

Most tissue classification models are untargeted and based on molecular patterns within the detection range of lipid species (e.g. glycerophospholipids, triglycerides). Several classification models were created based on PCA/LDA including either all the tissues or a subsets of animal/tissue types were created (Figure 35-Figure 36-Figure 37). 


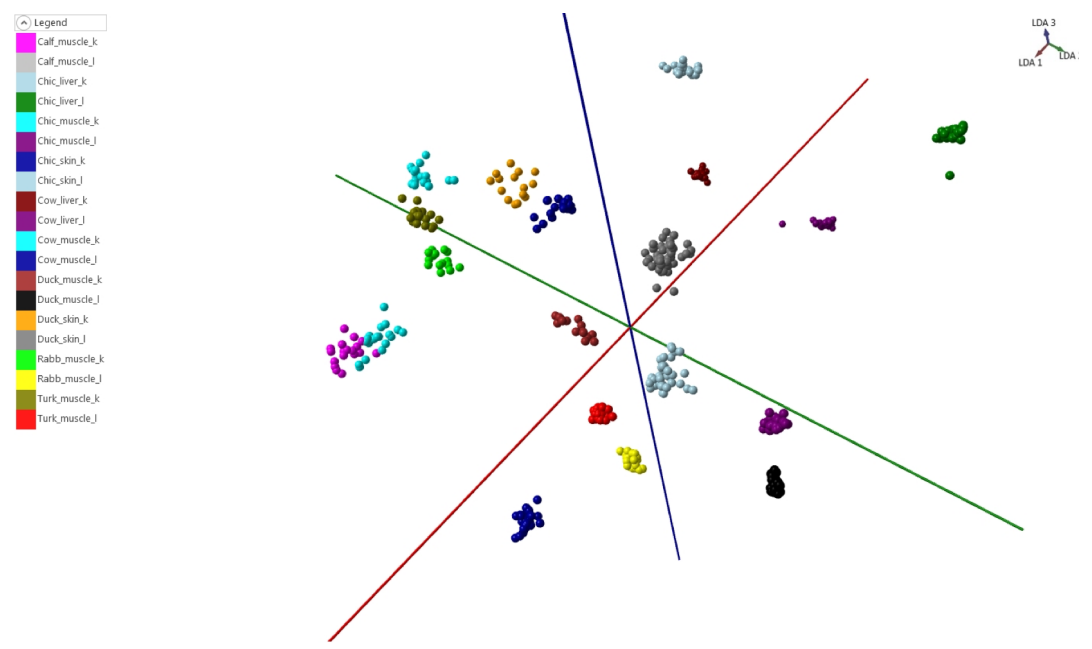

Figure 35 - REMS-LDA-3D score plot including all data acquired from sampling soft tissue types. $L=l a s e r$. k=diathermic knife.
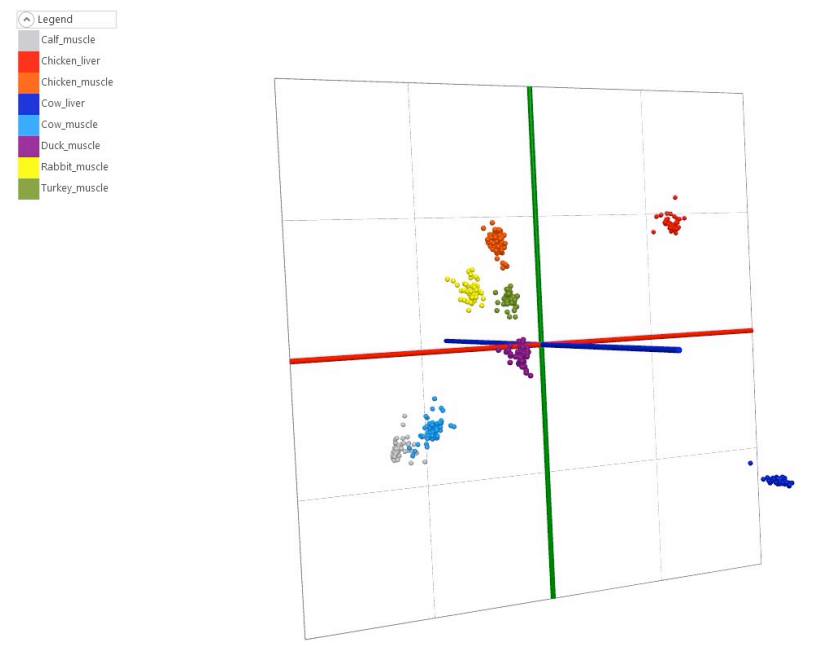

$T_{\text {DAA }}$

Figure 36 - Laser REMS-LDA-3D score plot including data acquired from sampling soft tissue types but excluding the data related to duck skin. Laser data only. 

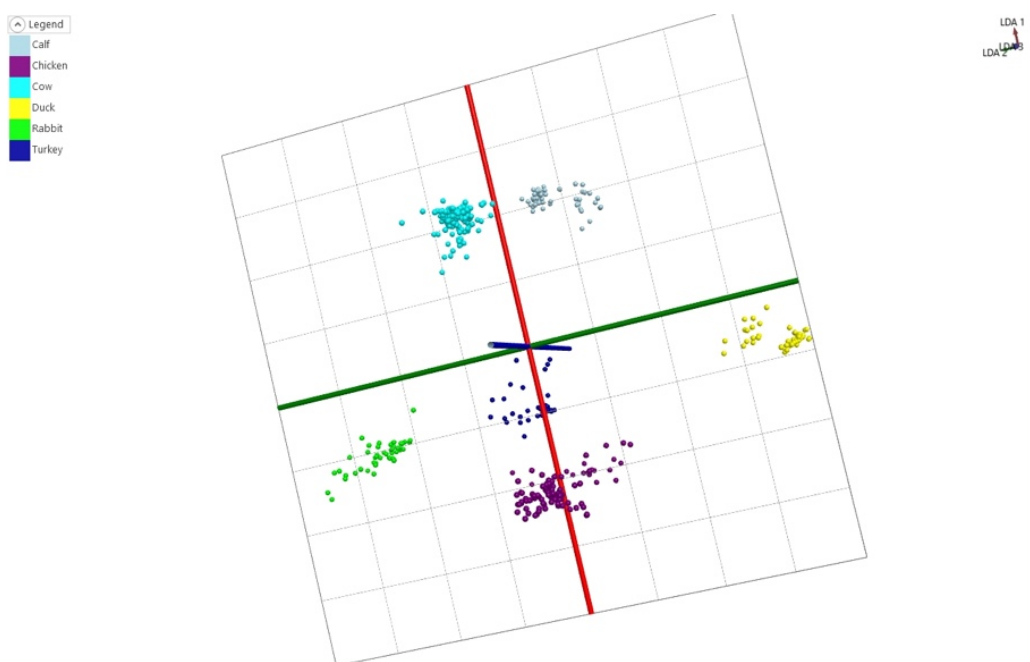

Figure 37 - Laser REMS-LDA-3D score plot including data acquired from sampling soft tissue types but excluding the data related to duck skin and duck liver. Diathermic data only.

This allowed the discrimination between tissues and the exclusion of outliers. The models were then subjected to a series of cross-validations where a model generated with the data from one handpiece was used to classify the data generated with the other handpiece; and vice versa. As reported in Table 22, the cross-validation using all tissue samples and animals show that it is possible to correctly classify only $58.64 \%$ of the tissue samples when using the diathermic knife-based classification/prediction model to classify data from the laser. The classification rate accuracy decreased to $28.73 \%$ when using the laser-based model to classify the data from the diathermic knife. The cross-validation using one tissue sample (either muscle or liver) from two animals, led to a $100 \%$ classification rate for most of the tissues (with the exception for turkey/duck muscle) when using the diathermic knifebased classification/prediction model to classify data from the laser. This classification rates is halved (52\%) when performing the cross-validation in the opposite manner (Table 22). 
Table 22 - Percentage of well-recognized tissue after cross-validation. The first column represents the tissue type/animal included in their respective model. The second and third columns represent the results of the crossvalidation when the classification model was built with data generated with the diathermic knife and used to classify the data acquired with the laser. The fourth and fifth columns represent the results of the cross-validation when the classification model was built with data generated with the laser and used to classify the data acquired with the diathermic. Mol. fin. = classification model based on molecular fingerprints

\section{Cross-validation}

\begin{tabular}{|c|c|c|c|c|}
\hline \multirow{2}{*}{$\begin{array}{l}\text { Data used to create the model } \\
\text { Model used to classify the data } \\
\text { acquired with: }\end{array}$} & \multicolumn{2}{|c|}{ Diathermic knife } & \multicolumn{2}{|c|}{ Laser } \\
\hline & \multicolumn{2}{|c|}{ Laser } & \multicolumn{2}{|c|}{ Diathermic knife } \\
\hline $\begin{array}{l}\text { Animal/tissue included in the } \\
\text { model }\end{array}$ & $\begin{array}{l}\text { Mol. fin. Based } \\
\text { model }\end{array}$ & $\begin{array}{c}\text { Peak list-based } \\
\text { model }\end{array}$ & $\begin{array}{l}\text { Mol. fin. Based } \\
\text { model }\end{array}$ & $\begin{array}{c}\text { Peak list-based } \\
\text { model }\end{array}$ \\
\hline All ** & $58.640 \%$ & $70.890 \%$ & $28.730 \%$ & $55.830 \%$ \\
\hline All no skin & $67.440 \%$ & $86.940 \%$ & $55.250 \%$ & $66.940 \%$ \\
\hline Chicken * & $59.960 \%$ & $92.312 \%$ & $59.650 \%$ & $67.590 \%$ \\
\hline Chicken / Duck (muscle) & $100.000 \%$ & $90.310 \%$ & $50.000 \%$ & $52.940 \%$ \\
\hline Chicken / Turkey (muscle) & $100.000 \%$ & $100.000 \%$ & $51.350 \%$ & $84.360 \%$ \\
\hline Chicken / Cow (liver) & $100.000 \%$ & $100.000 \%$ & $52.630 \%$ & $78.250 \%$ \\
\hline Chicken / Cow (muscle) & $100.000 \%$ & $100.000 \%$ & $53.350 \%$ & $100.000 \%$ \\
\hline Chicken / Calf (muscle) & $100.000 \%$ & $100.000 \%$ & $52.470 \%$ & $100.000 \%$ \\
\hline Chicken / Rabbit (muscle) & $100.000 \%$ & $100.000 \%$ & $54.850 \%$ & $100.000 \%$ \\
\hline Cow * & $77.780 \%$ & $98.450 \%$ & $52.680 \%$ & $76.390 \%$ \\
\hline Cow / Calf (muscle) & $100.000 \%$ & $100.000 \%$ & $52.120 \%$ & $100.000 \%$ \\
\hline Cow / Rabbit (muscle) & $100.000 \%$ & $100.000 \%$ & $51.540 \%$ & $100.000 \%$ \\
\hline Calf / Rabbit (muscle) & $100.000 \%$ & $100.000 \%$ & $52.110 \%$ & $100.000 \%$ \\
\hline Turkey / Duck (muscle) & $50.000 \%$ & $56.360 \%$ & $50.000 \%$ & $100.000 \%$ \\
\hline Turkey / Cow (muscle) & $100.000 \%$ & $100.000 \%$ & $51.130 \%$ & $100.000 \%$ \\
\hline Duck / cow (muscle) & $100.000 \%$ & $100.000 \%$ & $52.140 \%$ & $100.000 \%$ \\
\hline Duck / rabbit (muscle) & $100.000 \%$ & $100.000 \%$ & $53.960 \%$ & $100.000 \%$ \\
\hline
\end{tabular}

Based on these results, building a model from the diathermic data and subsequently classify the laser data provides a more accurate classification rate. A plausible explanation lies in the difference in precision and therefore variation between the data generated by the two handpieces (Figure 38a and Figure 39). The diathermic measurements are less precise, causing a higher 
variance in the predictive model (Figure 39). This results in a lower chance to obtain outliers when classifying data from the laser and therefore in a higher score for the cross-validation with the laser data $[185,186]$. Training a model from the data obtained with the laser creates a model with less variance which is more prone to generating outliers when cross-validating with less precise data (Figure 39).

We here recommend building unique classification models for the two handpieces on the diathermic data, only if the data is well segmented. The higher variance of the data from the diathermic knife could otherwise lead to misclassification of tissues. The classification performances of the models can be improved by introducing targeted features, as reported in the next paragraph.

\section{Introduction of a targeted features to improve classification performance.}

An ideal situation with real-time tissue classification would be to discriminate different tissue types based on a classification database independently from the handpiece used. Models including tissue-specific peak lists have recently proven to lead to better classification rates [175]. Several classification/prediction models were created based on PCA-LDA with a maximum number of one tissue type and two animals (e.g. cow [liver] vs chicken [liver], Figure 38) analyzed with both the $\mathrm{CO}_{2}$ laser and the diathermic knife. We limited these models to one tissue type and two animals in order to have a total number of four variables in the score plot. From the loading plot of generated classification models (based on molecular fingerprints), we searched for similarities in the spectra between the two handpieces for the same tissue type. We focused on characteristics peaks able to discriminate the two tissue types independently from the handpiece used for sampling. 


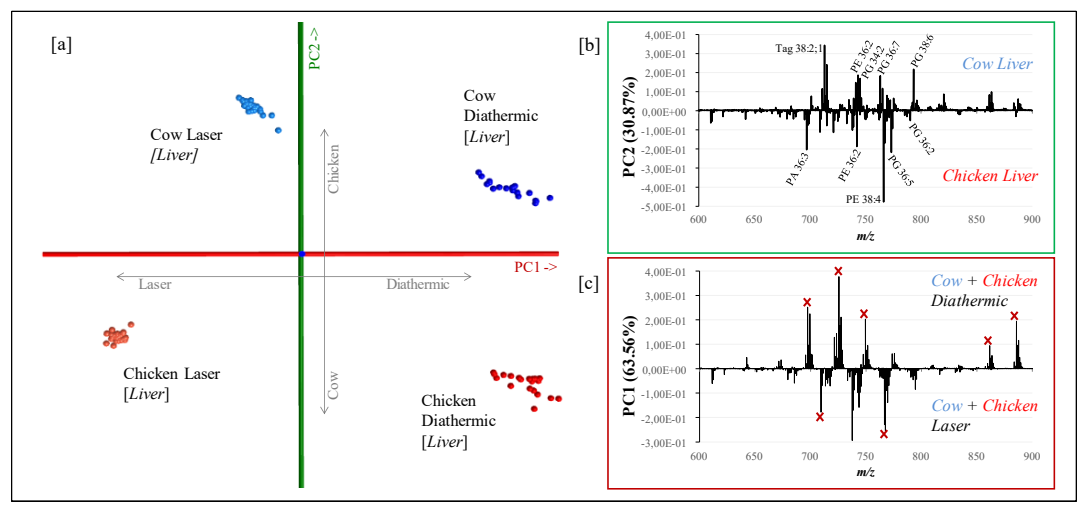

Figure 38 - A PCA-LDA predictive model used for the creation of inclusion and exclusion peak lists. [a] Score plot from a model including cow liver and chicken liver analyzed with the two handpieces. From the loading plot, peaks able to discriminate between the two tissue types [b] were selected as an "inclusion list" (referred to as "Inc."). Peaks able to discriminate between the two handpieces [c] were selected as an "exclusion list" (referred to as "Excl. "). The two peak lists were compared, and the peaks included in the exclusion list were removed from the inclusion list in order to create tissue-specific peak lists (tissue-specific peak list = Inc. - Excl.).

In order to create a unique peak list for the model, an inclusion and an exclusion peak lists were generated:

A tissue-related peak lists with peaks that contributed the most to differentiating between tissue-types (Inclusion list, green component in Figure 38).

A handpiece-related peak list with peaks that contributed the most to differentiate samples based on the handpieces (Exclusion list, red component in Figure 38).

These two peak lists were compared and combined as follows: peaks from the exclusion list were removed from the inclusion list to create several tissue-specific peak lists, as well as a combined list containing 50 peaks which contributed the most to the separation of the tissue types independently from the handpiece. We found that in this specific application including less or more than 50 peaks reduce the accuracy of the classification rate for every model. As reported in Table 22, the PCA/LDA peak list-based model led to an improvement for almost all the animal comparisons, with the exception of the models that included duck muscle. An improvement from 59\% to $71 \%$ good classification in 
the cross-validation when leaving the "laser out" for the classification of the diathermic data was observed when all tissue samples were included. When excluding skin from the model, there was an improvement from $67 \%$ to $87 \%$. Additionally, leaving the "diathermic out" led to an improvement from 29\% to 56\% in the prediction rate with all the tissues included, and an improvement from 55\% to 67\% when excluding the data acquired from the skin samples (Table 22). This can be attributed to oversampling. During skin sampling with both handpieces, some of the smoke generated actually belonged to the muscle beneath the skin. Additionally, the skin of selected animals had a very heterogeneous composition (e.g. epidermis, derma, fat) while being extremely thin. All these factors increased the variance of the skin data and for these reasons, we decided to exclude this tissue from the classification model.

The data clearly show that the peak list-based model leads to higher classification rates. As observed in the previous paragraph, a larger variation of the data was observed when sampling with the diathermic knife. The introduction of a tissue-specific peak list led to a higher improvement in the classification rate of the model built with the laser data for similar reasons (Figure 38a/Figure 39).

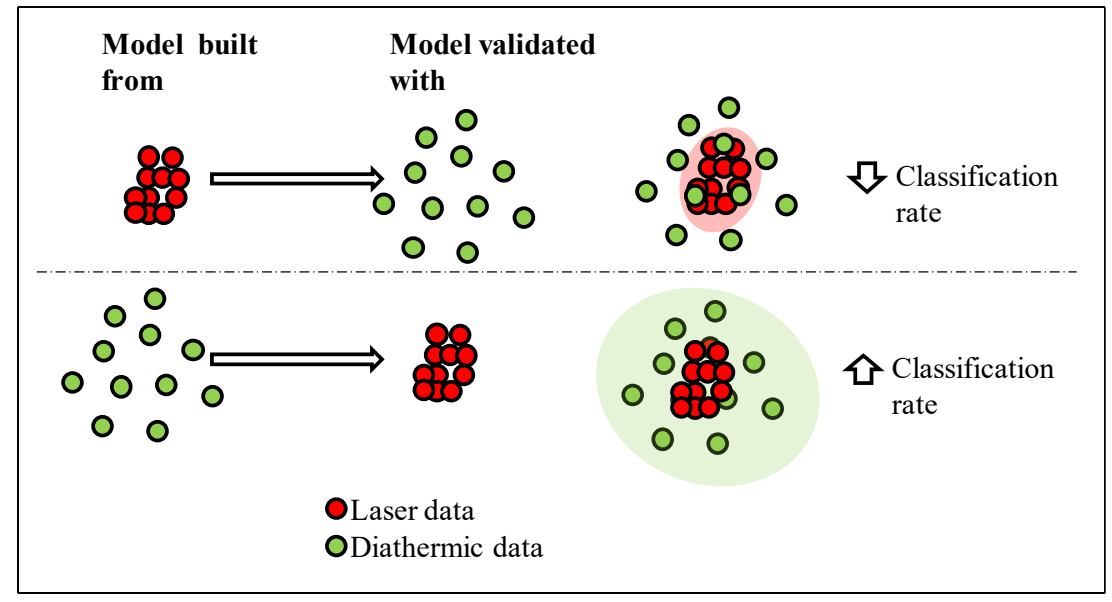

Figure 39 - Schematic interpretation of the influence of the data variation in the creation of a predictive model. The data not included in the ellipse (represented but the light-colored ovals) are those which returned as outliers by the crossvalidation process. This illustrates why the use of a more precise model leads to more outliers when classifying a subsequent dataset which inherently comprises more variance. 
The larger variation of the data from the diathermic knife (green data in Figure 39) caused a higher variance in the predictive model which results in a higher score during the cross-validation with the laser (low data variation, red data in Figure 39) $[185,186]$. Training a model from the data obtained with the laser creates a model with less variance. Validating this last model with the data from the diathermic knife even if more precise and accurate, results in an overall lower classification rate.

\section{Analysis of hard tissues}

We investigated the possibility of analyzing hard tissues with the laser handpiece. Since malignancies such as soft tissue sarcoma can occur near bones, collecting information from hard tissue in the context of this disease might help with diagnosis and identifying tumor margins. Ultimately this would provide a better understanding and improve outcome of these conditions. Soft tissue sarcomas may rest against the periosteal surface and invade the bone [187-193]. Sampling the bone in vivo with limited invasiveness can be crucial for a better prognosis or choice of therapy $[194,195]$. Furthermore, the analysis of hard tissue could also contribute to a better understanding of the healing process in response to bone fractures. Not all bones heal from a fracture and if the fracture occurs near a joint, post-traumatic osteoarthritis can develop [196-198]. The diathermic knife cannot be used to sample dry tissue such as bone or cartilage as it is simply not possible to cut through such tissues with this handpiece due to its physical properties. Furthermore, electrosurgical dissection is based on the joule heating to evaporate of tissues by an electric current [173] which cannot be achieved in tissue that poorly conducts heat, such as bone. Therefore, we assessed the possible use of the CO2 laser in acquiring molecular profiles from bone and cartilage. We demonstrated that the laser allowed the generation of lipid-rich smoke from hard tissues like bone 
and cartilage. Figure 40displays the spectra generated from bone, cartilage and bone marrow from a chicken leg.

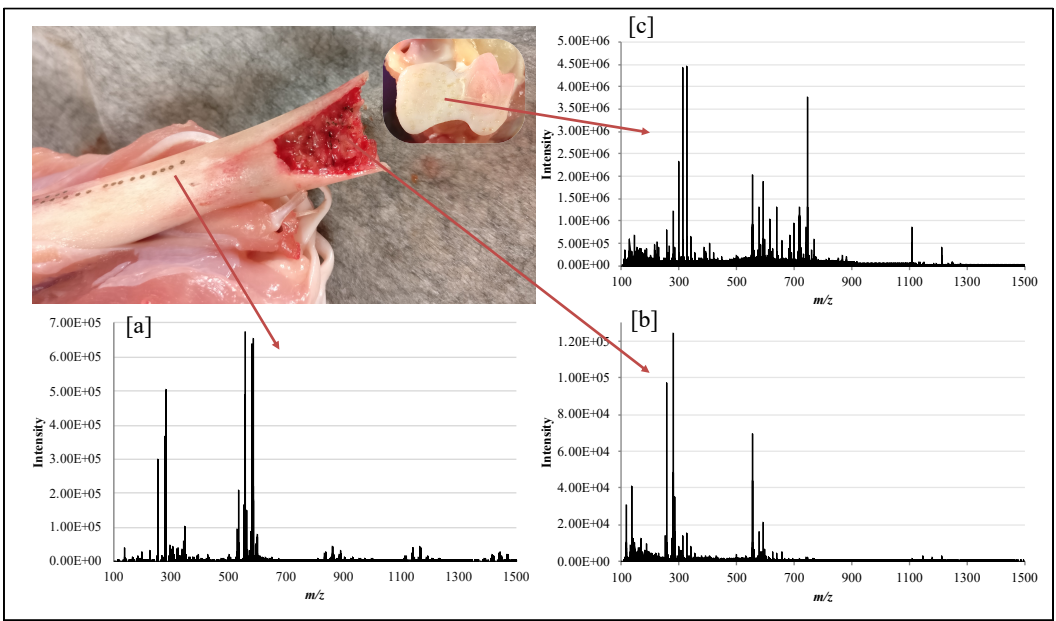

Figure 40 - Sampling of hard tissue with the laser-REIMS system. With the $\mathrm{CO}_{2}$-laser it was possible to generate a signal from bone [a], bone marrow [b] and cartilage [c]. It is not possible to generate aerosols and consequently a signal while using the diathermic knife due to the hard nature of the bone.

The best results were obtained using a laser power of $25 \mathrm{~W}$ for the bone marrow and cartilage and $30 \mathrm{~W}$ for the bone with "pulse modality" of $0.3 \mathrm{~s}$ and $0.4 \mathrm{~s}$ respectively. This indicates that an "ablative" pulse il required for the ejection of aerosols from hard tissues. No signal was obtained with power below $25 \mathrm{~W}$ or with the "continuous wave" modality (Figure 41). 


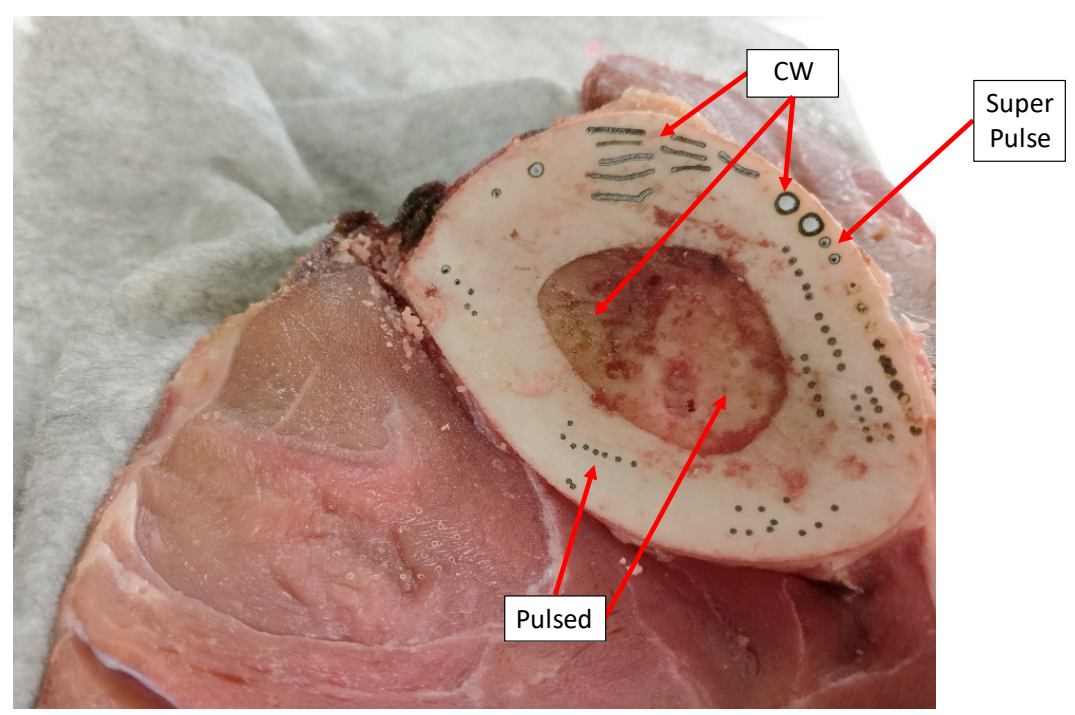

Figure 41 - Visualization of bone damage induced by laser sampling using different modalities available with the $\mathrm{CO}_{2}$ laser system. CW = continuous wave operated in laser dot modality.

Additionally, in order to generate any signal, the continous wave required an exposure time and power of the laser which caused a complete penetration of the chicken leg. Hence, it is not recommended to use the continous wave to sample hard tissue. The "super-pulse" modality created deeper holes in the tissues without generating any signal. Finally, it is notworthy that leaving the bone and cartilage inside the biosafety cabinet dried the tissues leading to a decrease of the signal over time. Hence, the ex vivo analysis of hard tissues must be performed in the shortest possible time after collection. We foresee that this will not be a limitation for future in vivo applications. 


\subsection{Chapter conclusions}

This work reports a comparison between a diathermic knife and a $\mathrm{CO}_{2}$ laser used as handpieces and coupled to a REIMS-TOF mass spectrometer for instantaneous tissue classification. The laser produced less tissue damage when compared to the diathermic knife. The higher reproducibility of the laser was partly due to the fixed position and controlled ablation time. It was not possible to mount the diathermic knife onto the same robotic arm because, at its current state, the arm cannot raster or be programmed for electrocautery applications. The laser was able to generate aerosols rich in lipids from hard tissues such as bone, bone marrow and cartilage tissue, which extends the field of applications of REIMS.

Furthermore, we demonstrated that the data generated with one sampling modality can be employed to classify the data generated with the other handpiece, and vice versa, with the introduction of a model based on peak lists that are tissuespecific and common to the two handpieces. We confirm that peak-based classification models considerably increase the correct classification rate, in comparison to when only the entire molecular pattern is considered.

Combined, these findings strengthens the valuability and versatility of REIMS for instantaneous identification/classification of soft and hard tissues and the coupling to different handpieces generating aerosol expands its field of application. We foresee that this approach can be easily translated to clinical applications since the two handpieces used in this work are approved medical devices that are frequently used for surgical procedures. 



\section{Chapter 6}

Epilogue

Epilogo

結語

Epilogue

Epilog

Epiloog

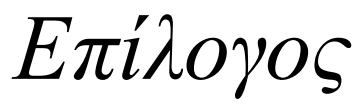

Epílogo

ảềổốệ

Épilogue

ýĹ́śśñ

אֶּפילוֹג

エピローグ

结语

발문 


\section{$6 \quad$ Epilogue \\ 6.1 Summary}

This thesis presents several aspects and applications of MS as a diagnostic tool. It describes the possibility of analyzing different sample types (solid, liquid and gas) and how this powerful tool is used for quantification of substances, qualification of biologically relevant molecules within samples, locating molecules in tissue sections and combining MS with clinical tools to improve the outcomes of cancer patients.

Chapter 2 is a summary of MS applications for liquid biological samples (serum, blood and urine) developed for anti-doping veterinary purposes. The best MS technique allowing the quantification and qualification of selected substances in these matrices is liquid chromatography (precisely Ultra HighPressure liquid chromatography - UHPLC) coupled to a triple quadrupole mass spectrometer (QQQ) equipped with an Electrospray ionization source (ESI). The combination of UHPLC, ESI and QQQ allows the precise isolation, quantification and qualification of substances in complex matrices such as biological fluids.

LC-MS is a powerful tool for the analysis and quantification of substances in different matrices, but lacks spatial resolution. Chapter 3 is the perfect example of how different MS techniques can reveal hidden information within samples. In this chapter, the analysis of rat feces by LC-MS allowed a better understanding of the mechanism behind the cholesterol lowering effect of a lentil extract. From the matrix assisted laser desorption ionization (MALDI) mass spectrometry imaging (MSI) analysis of tissues harvested from the same rats, we discovered a synergistic mechanism behind the cholesterol lowering effect. MALDI-MSI granted the analysis of hard tissue while maintaining the spatial distribution of the molecules being analyzed. MALDI-MSI allowed us to 
qualify and locate the distribution of molecules within organs and tissues, revealing information not accessible when using LC-MS.

Chapter 4 focuses on tackling a well-known problem of MSI: the lack of proper quality control (QC) procedures. This chapter develops and compares a new QC tissue (pork fat) to a more conventional spot of cytochrome C. The pork fat was formalin fixed and paraffin embedded (FFPE), sliced and mounted next to breast cancer (BC) tissues on the same glass slide, and used to monitor the sample preparation and digestion efficiency. Because the pork fat is FFPE tissue, it undergoes the same sample preparation steps as the BC tissues. The application of FFPE pork fat as QC allowed the visualization of two types of BC patients and relative subtypes.

Lastly, Chapter 5 demonstrates how coupling different tools with a mass spectrometer can broaden the applications of MS. In this chapter, two types of scalpels (laser and diathermic) commonly used in surgical procedures were coupled to a MS and used as smoke generators for the real-time analysis and classification of tissues. The handpieces were compared and a unique classification model able to classify tissues was created and improved. 


\subsection{Samenvatting}

Dit proefschrift presenteert verschillende aspecten en toepassingen van MS als diagnostisch hulpmiddel. Het beschrijft de mogelijkheid van het analyseren van verschillende soorten monsters (vast, vloeibaar en gas) en hoe dit krachtige hulpmiddel wordt gebruikt voor de kwantificatie van monsters, kwalificatie van biologisch relevante moleculen in monsters, het lokaliseren van moleculen in weefselsecties en het combineren van MS met klinische hulpmiddelen om de uitkomsten van kankerpatiënten te verbeteren.

Hoofdstuk 2 is een samenvatting van de toepassingen van MS voor vloeibare biologische monsters (serum, bloed en urine) die zijn ontwikkeld voor veterinaire doeleinden tegen doping. De beste MS-techniek die de kwantificatie en kwalificatie van geselecteerde monsters in deze matrices mogelijk maakt is vloeistofchromatografie (Ultra High-Pressure liquid chromatography - UHPLC) gekoppeld aan een drievoudige quadrupol massaspectrometer (QQQ) uitgerust met een Electrospray ionisatiebron (ESI). De combinatie van UHPLC, $E S I$ en $Q Q Q$ maakt de nauwkeurige isolatie, kwantificatie en kwalificatie van stoffen in complexe matrices zoals biologische vloeistoffen mogelijk.

LC-MS is een krachtig instrument voor de analyse en kwantificering van monsters in verschillende matrices, maar mist spatiale resolutie. Hoofdstuk 3 is het perfecte voorbeeld van hoe verschillende MS-technieken verborgen informatie in monsters kunnen onthullen. De analyse van rattenontlasting door LC-MS in dit hoofdstuk zorgde voor een beter begrip van het mechanisme achter het cholesterolverlagende effect van een linze-extract. Van de matrix geassisteerde laser desorptie ionisatie. Door (MALDI) massaspectrometrie imaging (MSI) analyse van weefsel geoogst van dezelfde ratten ontdekten we een synergetisch mechanisme achter het cholesterolverlagende effect. MALDI-MSI verleende de analyse van hard weefsel met behoud van de spatiale verdeling van de te analyseren moleculen. MALDI-MSI stelde ons in staat om de verdeling van moleculen in organen en weefsels te kwalificeren en 
te lokaliseren, waardoor informatie werd onthuld die niet toegankelijk was bij gebruik van LC-MS.

Hoofdstuk 4 richt zich op het aanpakken van een bekend probleem van MSI: het ontbreken van procedures voor kwaliteitscontrole (QC). Dit hoofdstuk ontwikkelt en vergelijkt een nieuw QC-weefsel (varkensvet) met een meer conventionele plek van cytochroom C. Het varkensvet was in formaline gefixeerd en in paraffine ingebed (FFPE), gesneden en gefixeerd naast borstkanker (BC) weefsel op dezelfde glazen coupe om de monstervoorbereiding en de verteringsefficiëntie te controleren. Omdat het varkensvet FFPE-weefsel is, ondergaat het dezelfde monstervoorbereiding als de BC-weefsels. De toepassing van FFPE-varkensvet als QC maakte de visualisatie van twee soorten BC-patiënten en relatieve subtypen mogelijk.

Ten slotte laat hoofdstuk 5 zien hoe het koppelen van verschillende tools aan een massaspectrometer de toepassingen van MS kan verbreden. In dit hoofdstuk werden twee soorten scalpels (laser en diathermisch) die gewoonlijk worden gebruikt in chirurgische procedures gekoppeld aan een MS en gebruikt als rookgeneratoren voor de real-time analyse en classificatie van weefsels. De handstukken werden vergeleken en een uniek classificatiemodel dat weefsels kon classificeren werd gecreëerd en verbeterd. 


\subsection{General discussion}

Over the last few decades, mass spectrometry (MS) found a more permanent position not only in the field of chemistry but also within medicine. MS and mass spectrometry imaging (MSI) are gaining momentum towards their development and implementation for clinical and pre-clinical applications, as well as screening analysis. This became increasingly evident during the formulation of this thesis. Performing experiments in collaboration with a private company and exploring a tremendous amount of different MS applications in the M4I laboratory clearly demonstrated its versatility.

Instruments and software are becoming more user friendly, and protocols for specific analyses are being standardized and adopted in several routine procedures within hospitals and private and public laboratories. Nevertheless, MS remains a valuable but complex analytical technique that, like many other techniques, comes with challenges which every mass spectrometrist around the world is attempting to eradicate. This thesis adresses several essential topics and MS applications for diagnostic purposes.

\subsubsection{Quantification}

Precise and accurate quantification and qualification of compounds in biological matrices is sometimes complicated and time consuming. Private and public laboratories around the world need fast, robust and accurate protocols able to detect and quantify many compounds in the shortest possible time.

LC-MS is a powerful technique which can separate, qualify and quantify traces of compounds in several matrices, requiring relatively short and easy sample preparation. This is of upmost importance in doping control analysis. Chapter 2 demonstrates how LC-MS allows the detection and quantification of several (>15) steroidal compounds (endogenous and exogenous) in different biological matrices. 
The combination of chromatography with mass spectrometry grants the separation and isolation of multiple compounds in complex matrices such as biological fluids. Chromatography by itself would not be enough to precisely quantify and qualify these substances. When coupled to a triple quadrupole mass spectrometer equipped with ion sources such as ESI (electrospray ionization), it is possible to separate, ionize, filter and precisely detect and quantify traces of compounds in different fluids.

On the other hand, quantification of compounds using MSI is still challenging. While both MS and MSI have their strenghts and pittfals, a combination of the two techniques allow to overcome their limitations.

\subsubsection{Combination of different techniques}

While absolute quantification using LC-MS is possible, the quantification of endogenous or exogenous compounds using MSI is considered to be the 'holy grail'. Chapter 3 reports the importance of combining different techniques to have a more complete understanding of biological processes.

In this chapter, it is clear how LC-MS allowed precise quantification of substances in plasma and feces, revealing possible mechanisms behind the cholesterol lowering effect of a lentil extract. It was only when organs from the same animal were analyzed with MSI that a more complete picture was provided.

MSI allowed the visualization and localization of the distribution of substances detected within organs using LC-MS.

\subsubsection{Quality control}

The results of MS experiments can be influenced by uncontrollable variables such as instrument variability, humidity and temperature. Quality controls (QC) are widely used in LC-MS in order to ensure the correct interpretation of data. In Chapter 2, one or more deuterated internal standards were used to monitor instrument variability. Other parameters such as temperature and humidity were not taken into 
account due the sample preparation method used. These last two parameters can deeply influence the results of MSI analysis.

According to the literature, no QC procedures have been established for MSI. Without a suitable QC, uncontrollable variablers such as humidity, operator and sample preparation might influence the data. In order to translate MS and MSI applications to the clinic, standard, robust, precise and accurate procedures must be developed with proper QC strategies. For this reason, Chapter 2 uses deuterated internal standars to monitor the analaysis and overcome data misinterpretation and Chapter 4 investigates two possible QC strategies able to improve the quality of the data and outlier detection. Throughout, we investigated a widely adopted QC (cytochrome C) to monitor digestion efficiency and developed a new QC able to monitor both the digestion efficiency and sample preparation. The new QC allowed the visualization of features within the cohort of analyzed breast cancer tissues which would have otherwise been impossible if using Cytochrome C as QC.

\subsubsection{Perspectives}

Chapter 5 describes a completely different application of MS for in situ/quasi-instantaneous tissue classification. Ambient techniques, as reported in Chapter 5, can be used not only for clinical application but also for the analysis of food, cells, bacterial cultures and material sciences as well. This chapter strives to highlight how MS is still a technique in development and broaden its possible applications. 



\section{Chapter 7}

Valorization

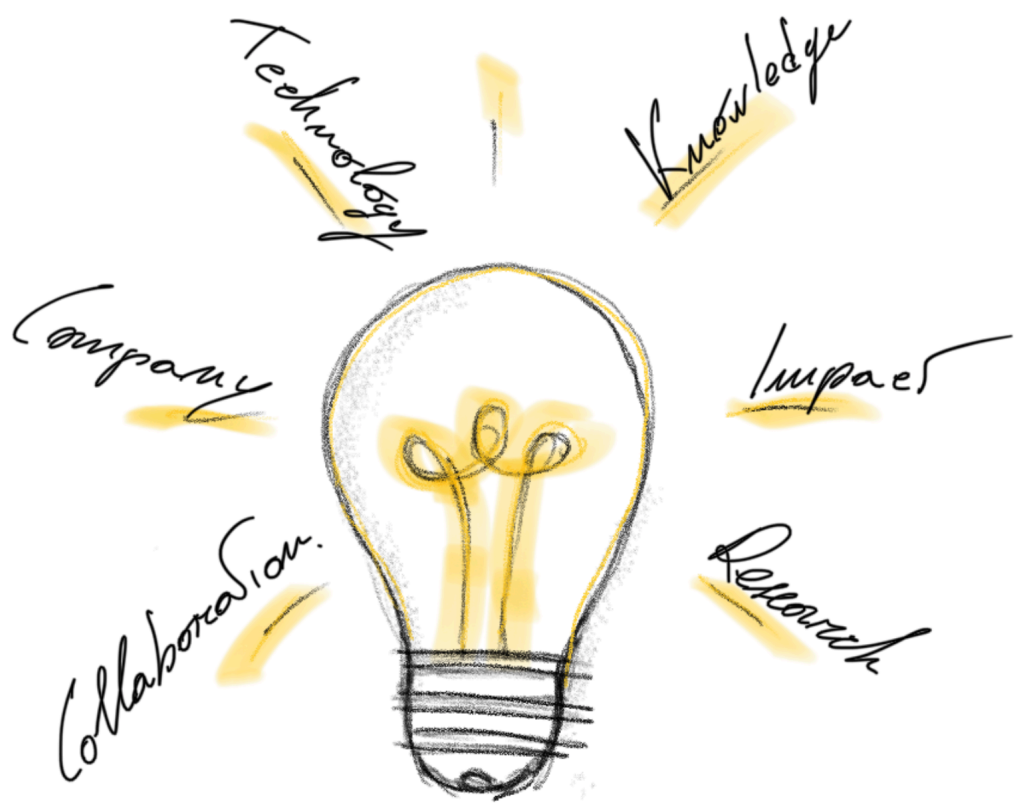




\section{$7 \quad$ Valorization}

This thesis sets out to explore and improve different aspects and applications of mass spectrometry as a device 'able to distinguish' (diagnostic tool) and characterize compounds in biological matrices in order to improve current methodologies.

This work pushes the boundaries of mass spectrometry and mass spectrometry imaging in terms of quantification, identification, analysis speed and coupling together of different techniques. These parameters are crucial for the applicability of mass spectrometry in clinical and non-clinical settings.

Constant improvement in methodology is crucial in all scientific fields, including MS and MSI. In this thesis, several protocols are improved in both the qualitative and quantitative aspects of MS/MSI.

Special care concerning the development of robust sample preparation and analytical procedures is required for mass spectrometry. This thesis firstly describes fast, robust and sensitive analytical procedures able to monitor the abuse of steroidal compounds in animal matrices (Chapter 2). These analytical methodologies were developed aiming to establish easy, robust and fast procedures for a market not yet fully regulated. These methodologies were not developed as a means to an end, but aimed to start a collaboration with a company which develops and produces ready-to-use diagnostic kits for the analysis of compounds in biological matrices. The procedures created have already been applied to several samples for doping control and will be developed into ready-to-use diagnostic kits by Eureka Lab division. 


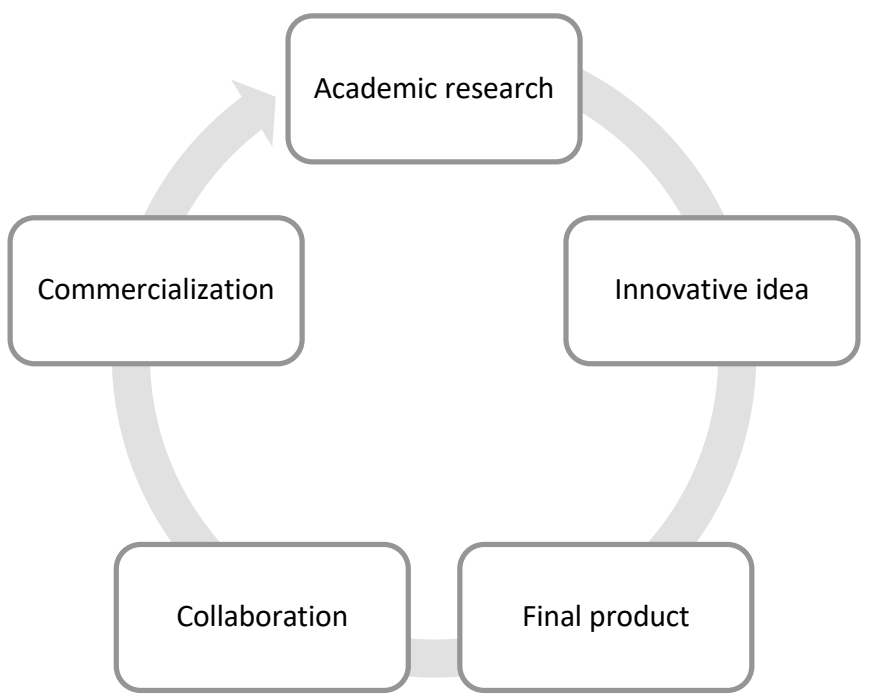

Figure 42 - Valorization circle

Liquid chromatography tandem mass spectrometry is a formidable technique able to precisely quantify and qualify substances in liquid matrices. However, it lacks spatial resolution. Chapter 3 is the perfect example of why combining two different mass spectrometric analyses is crucial for gaining a broader understanding of biological processes. In this chapter, the analysis of feces and plasma via UHPLCMS/MS provided an insight on how a lentil extract is able to lower the total cholesterol in rats. MALDI-MSI allows the precisely localization and qualification of lipids, bile acids and cholesterol within tissues. The MALDI analysis of organs harvested from the same animal sacrificed for the previous study not only confirmed the earlier findings but allowed for the understanding of the activation of a more complex biological pathway. After this joint research between M4I (NL) and the Unicam (IT), scientists from different fields in Unicam started seeking possible collaborations with M4I to expand their research. 

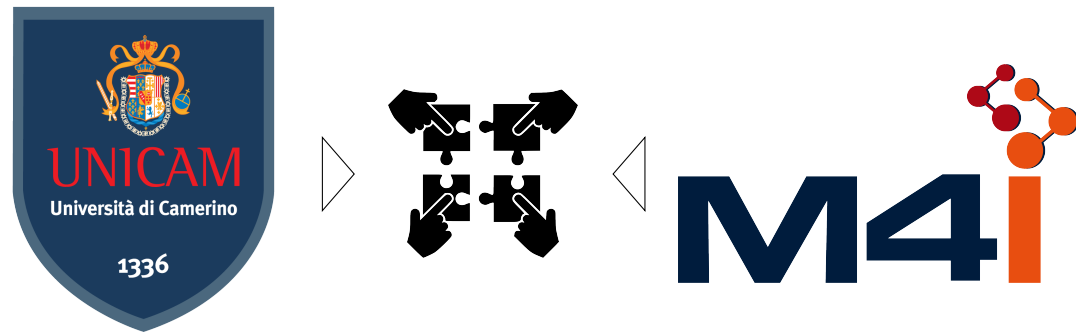

Figure 43 - Collaboration between M4l and Unicam.

Chapter 4 addressed a well-known problem for MALDI-MSI. The extensive sample preparation for FFPE tissues analyzed with MALDI-MSI can lead to artifacts in the results. Uncontrollable conditions such as humidity and temperature in the laboratory can influence the extraction or digestion of compounds from tissues. This chapter compares two innovative quality control QC methodologies able to monitor tissue digestion and peptide extraction of tissues. After the comparison, the QC was used to improve the classification of breast cancer tissues in order to prevent unnecessary surgery. The implementation of the QC led to a great improvement in the statistical analysis applied to these samples. Specifically, after filtering the samples using the QC, it was possible to use a prediction model to identify responders and nonresponders before treatment. This chapter acts as a pioneer in innovatively improving MALDI-MSI analysis.

Lastly, Chapter 5 describes mass spectrometry as a versatile tool for quasi-instantaneous tissue classification during surgery. Surgeons routinely use electrosurgery or laser scalpels in the operating room. These instruments generate smokes which can be aspirated and driven into a mass spectrometer thanks to the REIMS ionization source. The smoke is aspirated through a venturi pump after which it is thermally ionized and analyzed. Chapter 5 compares a $\mathrm{CO}_{2}$ surgical laser and a diathermic knife as handpieces coupled to a REIMS- 
TOF-MS. This shows the possibility to build a unique classification model for instantaneous tissue recognition ( $<3$ seconds). Additionally, the field of REIMS applications is expanded to hard tissue for in vivo surgical applications.

The work presented in this thesis is of interest to researchers working in biomedical and chemistry related fields. Mass spectrometry can complement and improve analytical methodologies routinely employed in clinical and non-clinical setups. Furthermore, the work reported includes collaborative studies involving research groups specialized in different scientific fields. By the combination of a broad range of expertise, this thesis presents novel MS approaches for the improvement of current diagnostic practices. 



\section{Chapter 8}

Appendices

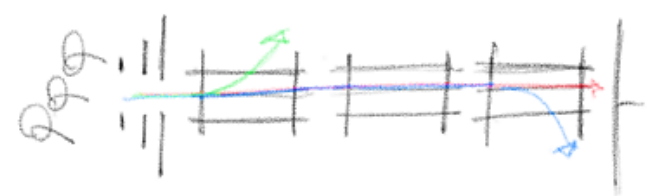

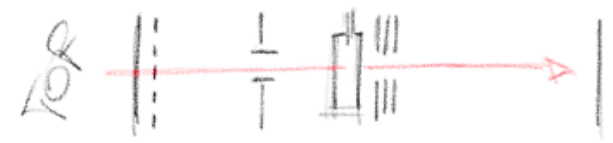
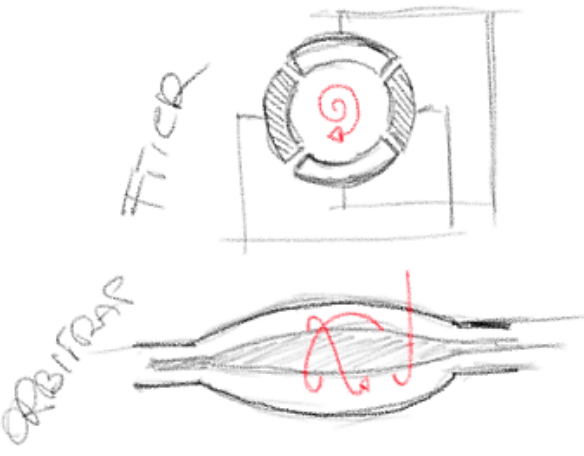


\section{Appendices}

\subsection{Tables}

Table A 1 - Content of endogenous and exogenous steroids in equine and bovine blood samples, expressed in ppb

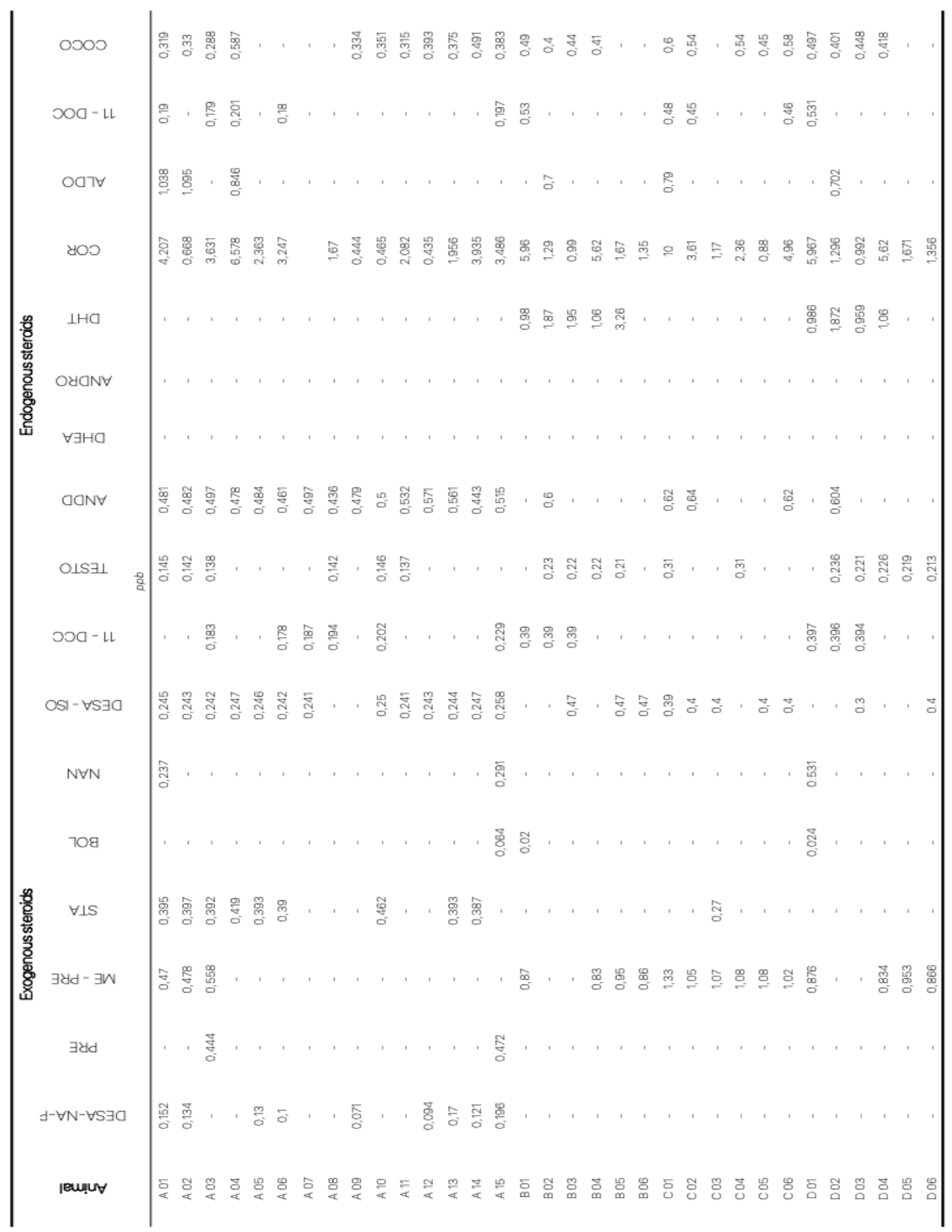


Table A 2 - Lipid identification. Prec. = Precursor ion, Theor. mass Theoretical mass (m/z)

\begin{tabular}{|c|c|c|c|c|c|c|c|c|c|}
\hline & & Solarix & Solarix & Synapt & Synapt & Synapt & & & \\
\hline Prec. & Theor.mass & $\begin{array}{l}\text { measured } \\
\mathrm{m} / \mathrm{z}\end{array}$ & ppm err. & $\begin{array}{l}\text { Prod. ion- } \\
\text { measured }\end{array}$ & Prod. ion theor. & ppm err. & Prod. ionid. & name & Adduct \\
\hline 714,51 & 714,507929 & 714,5078 & 0,18054 & $\begin{array}{l}279,23 \\
255,23\end{array}$ & $\begin{array}{l}279,232900 \\
255,232954\end{array}$ & $\begin{array}{l}10,38570 \\
11,57387\end{array}$ & $\begin{array}{l}F A 18: 2 \\
F A 16: 0(+0)\end{array}$ & PE (16:0_18:2) & {$[\mathrm{M}-\mathrm{H}]-$} \\
\hline \multirow[t]{4}{*}{833,52} & 833,518553 & 833,5183 & 0,30353 & $\begin{array}{l}279,23 \\
255,23\end{array}$ & $\begin{array}{l}279,232900 \\
255,232954\end{array}$ & $\begin{array}{l}10,38570 \\
11,57387\end{array}$ & $\begin{array}{l}\text { FA18:2 } \\
\text { FA16:0(+O) }\end{array}$ & $\mathrm{PI}\left(16: 0 \_18: 2\right)$ & {$[\mathrm{M}-\mathrm{H}]-$} \\
\hline & & & & 259,01 & 259,022443 & 48,04062 & $P \mid(259)$ & & \\
\hline & & & & 241,00 & 241,011878 & 49,28631 & $P \mid(241)$ & & \\
\hline & & & & 223,00 & 223,001313 & 5,88789 & $\mathrm{P} \mid(223)$ & & \\
\hline \multirow[t]{4}{*}{861,55} & 861,549853 & 861,5499 & $-0,05455$ & $\begin{array}{l}283,26 \\
279,26\end{array}$ & $\begin{array}{l}283,264254 \\
279,232900\end{array}$ & $\begin{array}{l}15,01800 \\
-97,04218\end{array}$ & $\begin{array}{l}\text { FA18:0(+0) } \\
\text { FA18:2 }\end{array}$ & $\mathrm{PI}\left(18: 0 \_18: 2\right)$ & {$[\mathrm{M}-\mathrm{H}]-$} \\
\hline & & & & 259,01 & 259,022443 & 48,04062 & $P \mid(259)$ & & \\
\hline & & & & 241,00 & 241,011878 & 49,28631 & $P \mid(241)$ & & \\
\hline & & & & 223,00 & 223,001313 & 5,88789 & $\mathrm{PI}(223)$ & & \\
\hline 766,54 & 766,539229 & 766,5393 & $-0,09262$ & $\begin{array}{l}303,22 \\
283,26\end{array}$ & $\begin{array}{l}303,232954 \\
283,264254\end{array}$ & $\begin{array}{l}42,72146 \\
15,01800\end{array}$ & $\begin{array}{l}\text { FA } 20: 4(+0) \\
\text { FA 18:0(+O) }\end{array}$ & PE (18:0_20:4) & {$[\mathrm{M}-\mathrm{H}]-$} \\
\hline 794,57 & 794,570529 & 794,5707 & $-0,21521$ & $\begin{array}{l}480,29 \\
283,26\end{array}$ & $\begin{array}{l}480,309563 \\
283,264254\end{array}$ & $\begin{array}{l}40,73164 \\
15,01800\end{array}$ & $\begin{array}{l}-\mathrm{FA} 22: 4(-\mathrm{H}) \\
\mathrm{FA} 18: 0(+\mathrm{O})\end{array}$ & PE (18:0_22:4) & {$[\mathrm{M}-\mathrm{H}]-$} \\
\hline \multirow[t]{4}{*}{857,52} & 857,518553 & 857,5186 & $-0,05481$ & $\begin{array}{l}303,22 \\
259,01\end{array}$ & $\begin{array}{l}303,232954 \\
259,022443\end{array}$ & $\begin{array}{l}42,72146 \\
48,04062\end{array}$ & $\begin{array}{l}\text { FA20:4(+0) } \\
\text { PI(259) }\end{array}$ & $\mathrm{PI}\left(16: 0 \_20: 4\right)$ & {$[\mathrm{M}-\mathrm{H}]-$} \\
\hline & & & & 255,23 & 255,232954 & 11,57387 & FA16:0(+0) & & \\
\hline & & & & 241,00 & 241,011878 & 49,28631 & $P \mid(241)$ & & \\
\hline & & & & 223,00 & 223,001313 & 5,88789 & $\mathrm{PI}(223)$ & & \\
\hline 885,55 & 885,549853 & 885,5499 & $-0,05307$ & $\begin{array}{l}303,22 \\
283,26\end{array}$ & $\begin{array}{l}303,232954 \\
283,264254\end{array}$ & $\begin{array}{l}42,72146 \\
15,01800\end{array}$ & $\begin{array}{l}\text { FA } 20: 4(+0) \\
\text { FA 18:0(+O) }\end{array}$ & $\mathrm{PI}\left(18: 0 \_20: 4\right)$ & {$[\mathrm{M}-\mathrm{H}]-$} \\
\hline \multirow[t]{3}{*}{769,53} & 769,502509 & 769,5024 & 0,14165 & $\begin{array}{l}303,22 \\
279,23\end{array}$ & $\begin{array}{l}303,232954 \\
279,232900\end{array}$ & $\begin{array}{l}42,72146 \\
10,38570\end{array}$ & $\begin{array}{l}\text { FA } 20: 4(+0) \\
F A 18: 2\end{array}$ & PG 36:4 & {$[\mathrm{M}-\mathrm{H}]-$} \\
\hline & & & & 255,23 & 255,232954 & 11,57387 & FA16:0(+0) & & \\
\hline & & & & 152,99 & 152,995834 & 38,13321 & PG(153) & & \\
\hline \multirow[t]{3}{*}{790,56} & 790,539229 & 790,5391 & 0,16318 & $\begin{array}{l}500,26 \\
480,29\end{array}$ & $\begin{array}{l}500,278263 \\
480,309563\end{array}$ & $\begin{array}{l}36,50702 \\
40,73164\end{array}$ & $\begin{array}{l}-F A 20: 2(-H) \\
-F A 22: 6(-H)\end{array}$ & PE 40:6 & {$[\mathrm{M}-\mathrm{H}]-$} \\
\hline & & & & 303,22 & 303,232954 & 42,72146 & FA20:4(+0) & & \\
\hline & & & & 283,26 & 283,264254 & 15,01800 & FA 18:0(+0) & & \\
\hline \multirow[t]{3}{*}{795,55} & 795,518159 & 795,5182 & $-0,05154$ & $\begin{array}{l}303,22 \\
283,25\end{array}$ & $\begin{array}{l}303,232954 \\
283,264254\end{array}$ & $\begin{array}{l}42,72146 \\
50,32304\end{array}$ & $\begin{array}{l}\text { FA } 20: 4(+0) \\
\text { FA 18:0(+O) }\end{array}$ & PG38:5 & {$[\mathrm{M}-\mathrm{H}]-$} \\
\hline & & & & 281,24 & 281,248604 & 30,59309 & FA $18: 1(+0)$ & & \\
\hline & & & & 152,99 & 152,995834 & 38,13321 & PG(153) & & \\
\hline \multirow[t]{3}{*}{881,54} & 881,518553 & 881,5184 & 0,17356 & $\begin{array}{l}480,29 \\
255,23\end{array}$ & $\begin{array}{l}480,309563 \\
255,232954\end{array}$ & $\begin{array}{l}40,73164 \\
11,57387\end{array}$ & $\begin{array}{l}\text {-FA22:6(-H) } \\
\text { FA16:0(+O) }\end{array}$ & $\mathrm{Pl}\left(16: 0 \_22: 6\right)$ & {$[\mathrm{M}-\mathrm{H}]-$} \\
\hline & & & & 241,00 & 241,011878 & 49,28631 & $P \mid(241)$ & & \\
\hline & & & & 223,00 & 223,001313 & 5,88789 & $\mathrm{Pl}(223)$ & & \\
\hline
\end{tabular}


Table A 3 - Lipid identification from REMS and MALDI-MS measurements of cow liver tissues. CE= collision energy (eV), Measured REMS = measured m/z with REMS-TOF system, Orbitrap = measured m/z with Orbitrap system, Theor. Mass = Theoretical mass ( $\mathrm{m} / \mathrm{z}$ ), pom err = calculated error in part-per-million (for REMS, Orbitrap), Prod ion = Production ( $m / z$, for REMS), Fragments id = fragments identification, Id = Lipid identification

\begin{tabular}{|c|c|c|c|c|c|c|}
\hline $\begin{array}{l}\frac{\breve{y}}{3} \\
\frac{7}{\sigma}\end{array}$ & $\begin{array}{l}\frac{T}{T} \\
\\
\sum\end{array}$ & 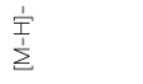 & $\sum_{\Sigma}^{\frac{1}{1}}$ & $\frac{\dot{1}}{\stackrel{1}{I}}$ & $\underset{\substack{\mathbf{1} \\
\Sigma}}{\frac{1}{1}}$ & \\
\hline 으 & $\begin{array}{l}\text { l'Z: } 8 \varepsilon \\
\text { be } e_{\perp}\end{array}$ & 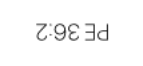 & Z:৮६৩d & $\begin{array}{l}\angle: 9 \varepsilon \\
\text { Эd }\end{array}$ & & 9:৪દ ৩১ \\
\hline 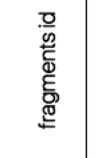 & 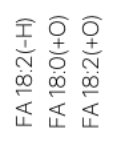 & 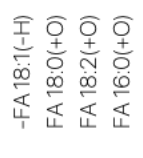 & 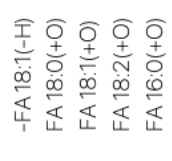 & 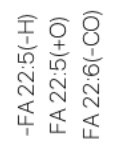 & 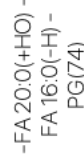 & 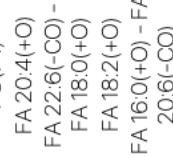 \\
\hline 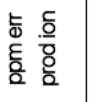 & 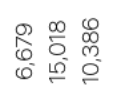 & 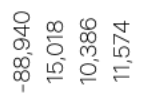 & 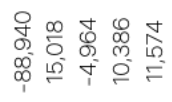 & 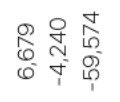 & 兽 & 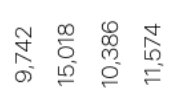 \\
\hline 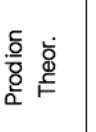 & 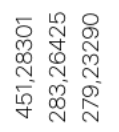 & 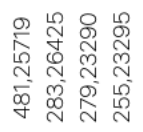 & 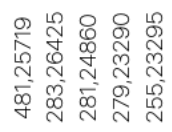 & 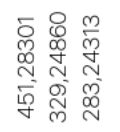 & $\begin{array}{l}\mathscr{8} \\
\stackrel{0}{0} \\
\stackrel{N}{1} \\
\bar{\infty} \\
\stackrel{+}{+}\end{array}$ & 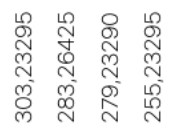 \\
\hline 흥 & 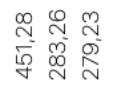 & 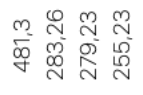 & 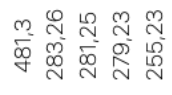 & 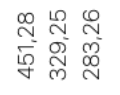 & 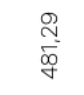 & 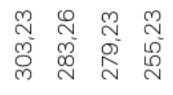 \\
\hline 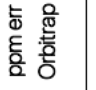 & $\begin{array}{l}\infty \\
0 \\
0 \\
0\end{array}$ & 周 & ర̃ & $\begin{array}{l}\stackrel{2}{f} \\
\text { f }\end{array}$ & $\begin{array}{l}\hat{0} \\
\stackrel{0}{0}\end{array}$ & \\
\hline 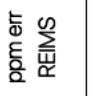 & 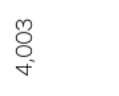 & $\begin{array}{l}\text { Dे } \\
\infty \\
\stackrel{్}{N}\end{array}$ & $\begin{array}{l}\stackrel{+}{+} \\
\infty \\
0 \\
0 \\
1\end{array}$ & 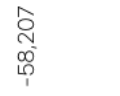 & 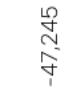 & \\
\hline 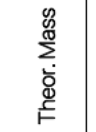 & 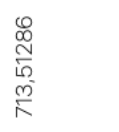 & 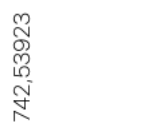 & 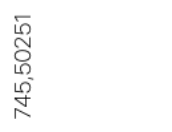 & 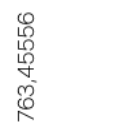 & 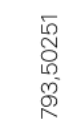 & \\
\hline 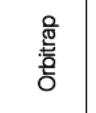 & 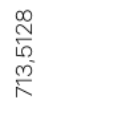 & $\begin{array}{l}\text { N } \\
\text { ON } \\
\text { N } \\
\text { N }\end{array}$ & 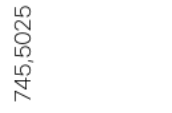 & 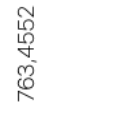 & 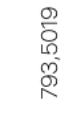 & \\
\hline 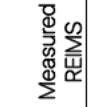 & $\frac{\sqrt[n]{n}}{\stackrel{M}{\Gamma}}$ & $\begin{array}{l}\text { N } \\
\text { N } \\
\text { N }\end{array}$ & 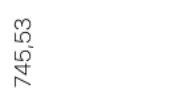 & 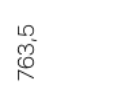 & 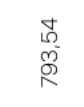 & \\
\hline 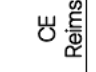 & $\stackrel{\infty}{N}$ & ஜे & প্ & ஓ & $\stackrel{m}{m}$ & \\
\hline
\end{tabular}


Table A 4 - Lipid identification from chicken liver measurements. CE= collision energy (eV), Measured REMS = measured $m / z$ with REMS-TOF system, Orbitrap = measured m/z with Orbitrap system, Theor. Mass = Theoretical mass (m/z), Ppm err = calculated error in part-per-million (for REIMS, Orbitrap), Prod ion = Product ion (m/z, for REIMS), Fragments id = fragments identification, $l d=$ Lipid identification

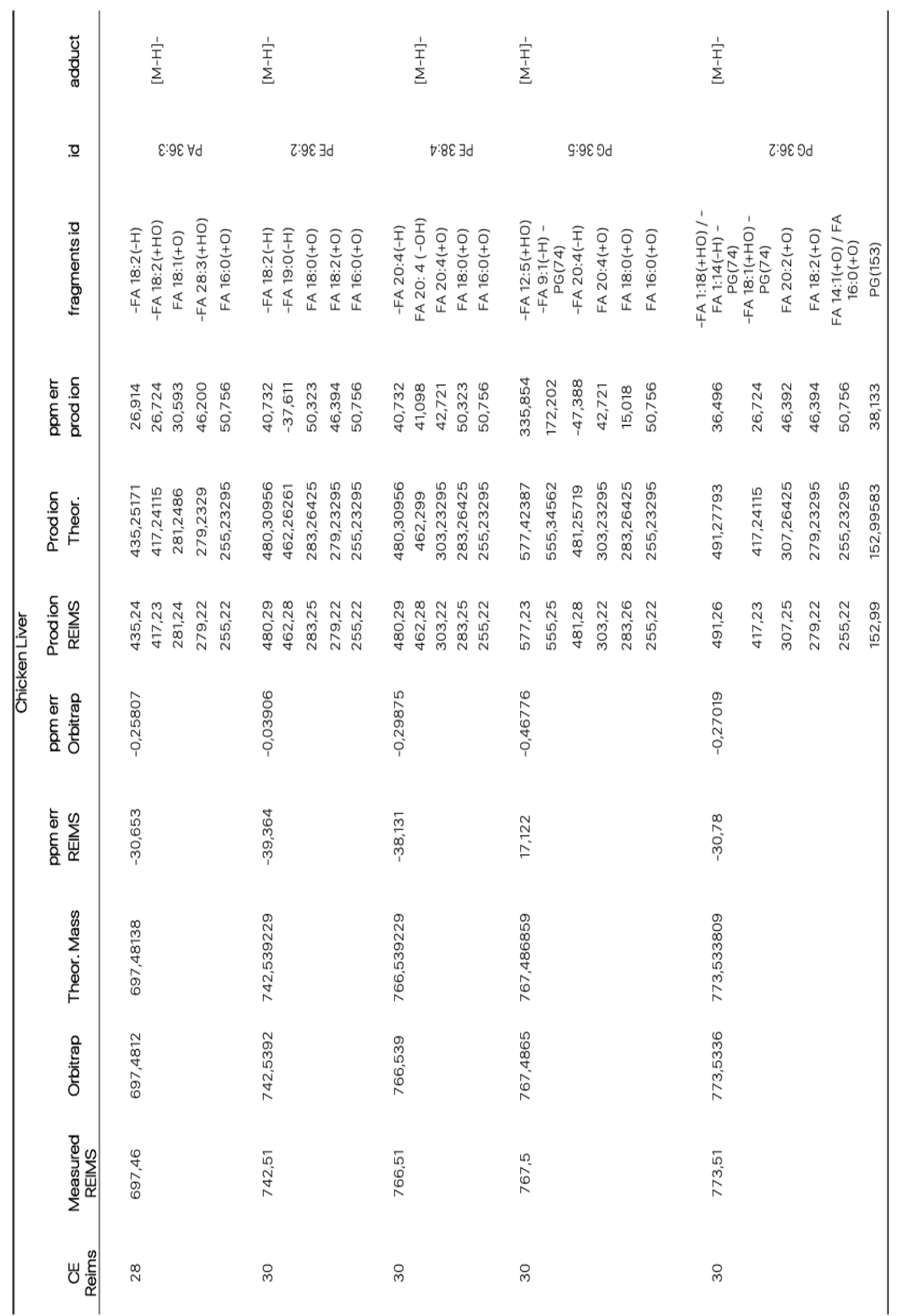


Table A 5 - Lipid identification from cow muscle measurements. CE= collision energy (eV), Measured REMS = measured $\mathrm{m} / \mathrm{z}$ with REMS-TOF system, Orbitrap = measured $\mathrm{m} / \mathrm{z}$ with Orbitrap system, Theor. Mass = Theoretical mass $(m / z)$, Ppm err = calculated error in part-per-million (for REMS, Orbitrap), Prod ion = Product ion ( $m / z$, for REMMS), Fragments id = fragments identification, Id = Lipid identification

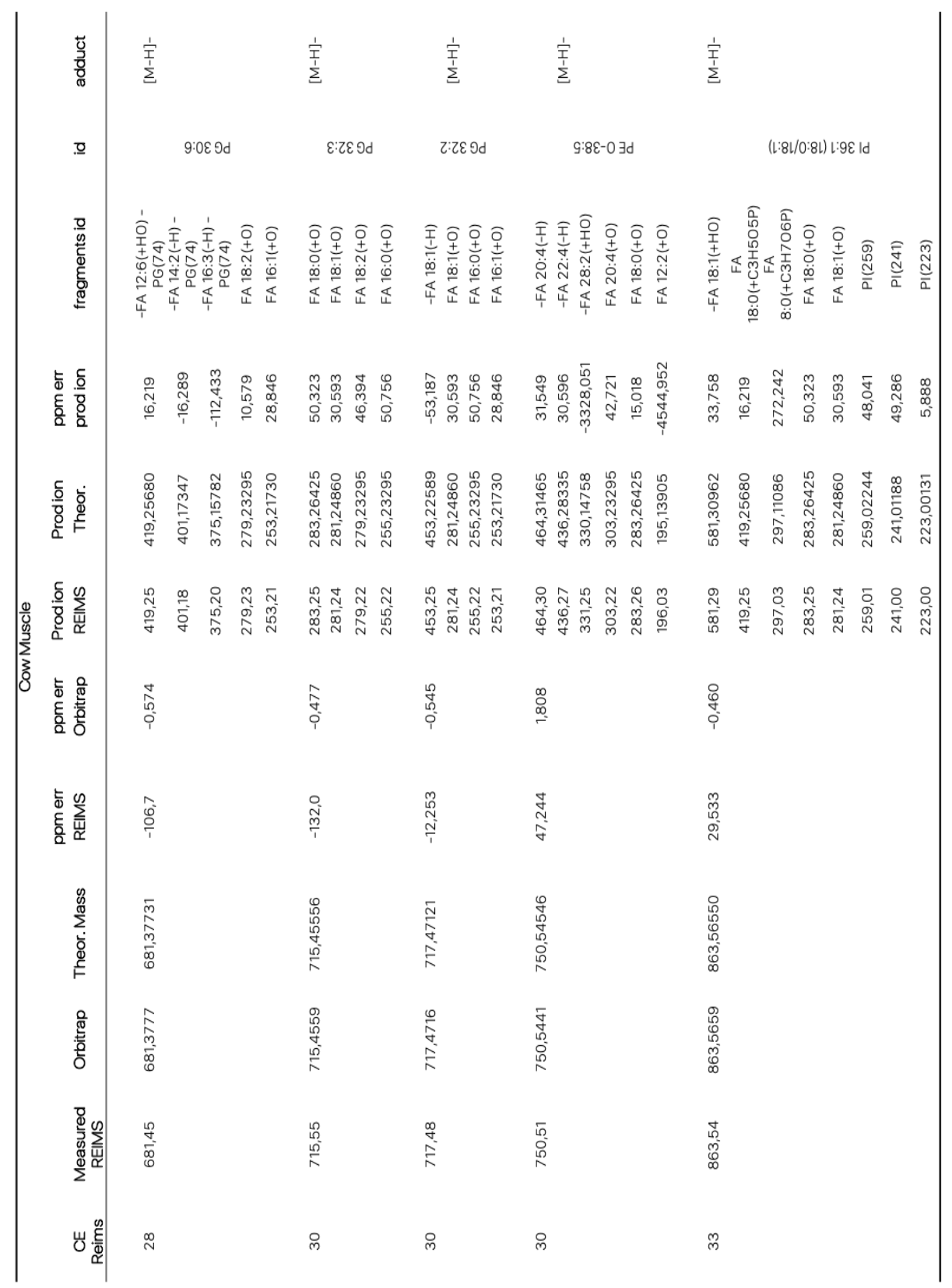


Table A 6 - Lipid identification from chicken muscle measurements. CE= collision energy (eV), Measured REIMS = measured $m / z$ with REMS-TOF system, Orbitrap = measured $m / z$ with Orbitrap system, Theor. Mass = Theoretical mass $(m / z)$, Ppm err = calculated error in part-per-million (for REMS, Orbitrap), Prod ion = Product ion ( $m / z$, for REIMS), Fragments id = fragments identification, ld = Lipid identification

\begin{tabular}{|c|c|c|c|c|}
\hline $\begin{array}{l}\frac{\mathrm{U}}{\mathrm{g}} \\
\frac{\mathrm{g}}{\mathrm{\sigma}}\end{array}$ & $\stackrel{\text { 宾 }}{\underline{\Sigma}}$ & $\frac{\dot{T}}{\sum}$ & $\stackrel{\text { 宾 }}{\underline{1}}$ & $\stackrel{\text { 宾 }}{\underline{\Sigma}}$ \\
\hline 으 & $\varepsilon: 9 \varepsilon \forall d$ & $Z: 9 \varepsilon \forall d$ & $(t: 0 z / 0: 8 L) \exists d$ & $(0: 8 \mathrm{l} / \mathrm{t}: 0 Z) \mathrm{Id}$ \\
\hline 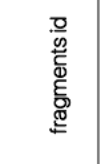 & 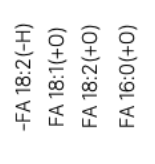 & 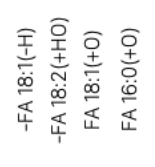 & 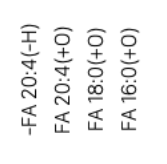 & 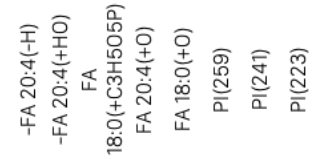 \\
\hline 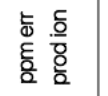 & 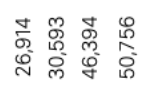 & 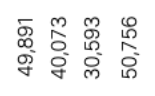 & 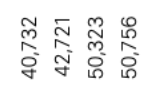 & 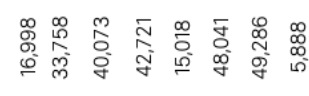 \\
\hline 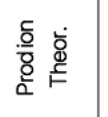 & 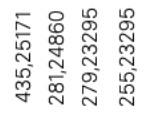 & 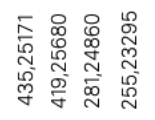 & 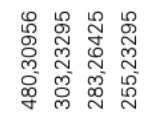 & 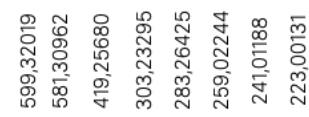 \\
\hline 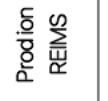 & 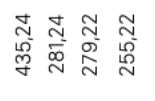 & 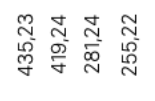 & 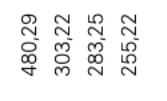 & 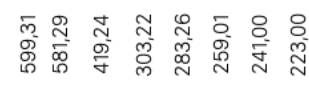 \\
\hline 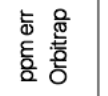 & 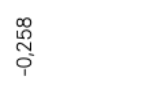 & 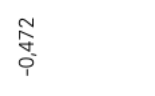 & $\begin{array}{l}\stackrel{8}{N} \\
\text { i }\end{array}$ & $\begin{array}{l}\circ \\
\stackrel{0}{0} \\
i \\
i\end{array}$ \\
\hline 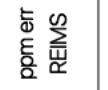 & 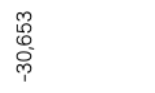 & 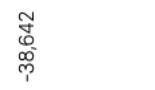 & $\underset{\substack{\frac{p}{\infty} \\
p}}{p}$ & 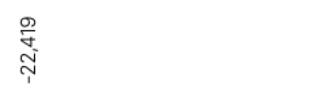 \\
\hline 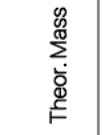 & 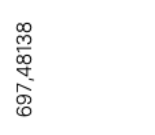 & 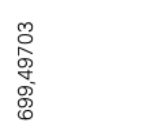 & 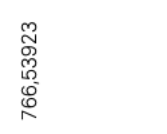 & 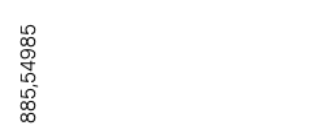 \\
\hline 遶 & 兑 & $\begin{array}{l}\hat{0} \\
\text { g. } \\
\text { oे } \\
\text { Oे }\end{array}$ & 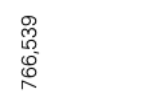 & 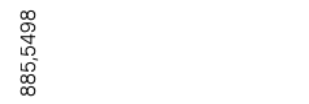 \\
\hline 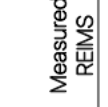 & $\begin{array}{l}\stackrel{0}{0} \\
\stackrel{0}{0}\end{array}$ & $\begin{array}{l}\text { f } \\
\text { o̊ } \\
\text { O }\end{array}$ & $\begin{array}{l}\overline{5} \\
0 \\
0 \\
0\end{array}$ & 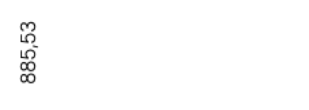 \\
\hline 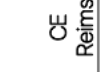 & $\stackrel{\infty}{N}$ & $\stackrel{\sim}{\sim}$ & 이 & m \\
\hline
\end{tabular}




\subsection{References}

1. Schalley, C.A., The expanding role of mass spectrometry in biotechnology Gary Siuzdak. Journal of the American Society for Mass Spectrometry, 2004. 15(4): p. 625-625.

2. Li, Y.Y., et al., Molecular Characterization of Dissolved Organic Matter and Its Subfractions in Refinery Process Water by Fourier Transform Ion Cyclotron Resonance Mass Spectrometry. Energy \& Fuels, 2015. 29(5): p. 2923-2930.

3. Monaco, S., Sistemi lineari Elementi di Analisi. 2011, Società Editrice Esculapio.

4. Griffiths, J., A brief history of mass spectrometry. Anal Chem, 2008. 80(15): p. 5678-83.

5. Adam, F., et al., Oxidative Desulfurization Process Monitoring Using Comprehensive Two-Dimensional Gas Chromatography and Fourier Transform Ion Cyclotron Resonance Mass Spectrometry. Energy \& Fuels, 2015. 29(4): p. 2312-2318.

6. Yamashita, M. and J.B. Fenn, Electrospray ion source. Another variation on the free-jet theme. The Journal of Physical Chemistry, 1984. 88(20): $\mathrm{p}$. 4451-4459.

7. Karas, M. and F. Hillenkamp, Laser desorption ionization of proteins with molecular masses exceeding 10,000 daltons. Analytical Chemistry, 1988. 60(20): p. 2299-2301.

8. Fenn, J.B., et al., Electrospray ion formation: Desorption versus desertion. Biochemical and Biotechnological Applications of Electrospray Ionization Mass Spectrometry, 1996. 619: p. 60-80.

9. Kellogg, M.D., Chapter 8 - Measurement of Biological Materials A2 Robertson, David, in Clinical and Translational Science (Second Edition), G.H. Williams, Editor. 2017, Academic Press. p. 137-155.

10. Fenn, J., et al., Electrospray ionization for mass spectrometry of large biomolecules. Science, 1989. 246(4926): p. 64-71.

11. Cech, N.B. and C.G. Enke, Practical implications of some recent studies in electrospray ionization fundamentals. Mass Spectrometry Reviews, 2001. 20(6): p. 362-387.

12. Koenig, J.L., Chapter 10 - Mass spectrometry of polymers, in Spectroscopy of Polymers (Second Edition). 1999, Elsevier Science: New York. p. 441480.

13. Seeley, E.H. and R.M. Caprioli, Molecular imaging of proteins in tissues by mass spectrometry. Proceedings of the National Academy of Sciences, 2008. 105(47): p. 18126-18131.

14. Caprioli, R.M., T.B. Farmer, and J. Gile, Molecular Imaging of Biological Samples: Localization of Peptides and Proteins Using MALDI-TOF MS. Analytical Chemistry, 1997. 69(23): p. 4751-4760. 
15. Stoeckli, M., et al., Imaging mass spectrometry: A new technology for the analysis of protein expression in mammalian tissues. Nature Medicine, 2001. 7(4): p. 493-496.

16. Chaurand, P., S.A. Schwartz, and R.M. Caprioli, Imaging mass spectrometry: a new tool to investigate the spatial organization of peptides and proteins in mammalian tissue sections. Current Opinion in Chemical Biology, 2002. 6(5): p. 676-681.

17. Chaurand, P., et al., New Developments in Profiling and Imaging of Proteins from Tissue Sections by MALDI Mass Spectrometry. Journal of Proteome Research, 2006. 5(11): p. 2889-2900.

18. Chughtai, K. and R.M.A. Heeren, Mass Spectrometric Imaging for Biomedical Tissue Analysis. Chemical Reviews, $2010.110(5)$ : p. 3237-3277.

19. McDonnell, L.A. and R.M.A. Heeren, Imaging mass spectrometry. Mass Spectrometry Reviews, 2007. 26(4): p. 606-643.

20. Ogrinc Potočnik, N., et al., Use of advantageous, volatile matrices enabled by next-generation high-speed matrix-assisted laser desorption/ionization time-of-flight imaging employing a scanning laser beam. Rapid Communications in Mass Spectrometry, 2015. 29(23): p. 2195-2203.

21. Harris, G.A., A.S. Galhena, and F.M. Fernández, Ambient Sampling/lonization Mass Spectrometry: Applications and Current Trends. Analytical Chemistry, 2011. 83(12): p. 4508-4538.

22. Schafer, K.C., et al., Real Time Analysis of Brain Tissue by Direct Combination of Ultrasonic Surgical Aspiration and Sonic Spray Mass Spectrometry. Analytical Chemistry, 2011. 83(20): p. 7729-7735.

23. Schafer, K.C., et al., In Situ, Real-Time Identification of Biological Tissues by Ultraviolet and Infrared Laser Desorption Ionization Mass Spectrometry. Analytical Chemistry, 2011. 83(5): p. 1632-1640.

24. Schafer, K.C., et al., In vivo, in situ tissue analysis using rapid evaporative ionization mass spectrometry. Angew Chem Int Ed Engl, 2009. 48(44): p. 8240-2.

25. Asha, S.K. and G. Sunitha, Effect of joule heating and MHD on peristaltic blood flow of Eyring-Powell nanofluid in a non-uniform channel. Journal of Taibah University for Science, 2019. 13(1): p. 155-168.

26. Ellis, T.L., et al., Nonthermal irreversible electroporation for intracranial surgical applications Laboratory investigation. Journal of Neurosurgery, 2011. 114(3): p. 681-688.

27. Bolt, F., et al., Automated High-Throughput Identification and Characterization of Clinically Important Bacteria and Fungi using Rapid Evaporative Ionization Mass Spectrometry. Analytical Chemistry, 2016. 88(19): p. 9419-9426.

28. Cameron, S.J.S., et al., Rapid Evaporative Ionisation Mass Spectrometry (REIMS) Provides Accurate Direct from Culture Species Identification within the Genus Candida. Scientific Reports, 2016. 6. 
29. Monge, M.E., et al., Mass Spectrometry: Recent Advances in Direct Open Air Surface Sampling/Ionization. Chemical Reviews, 2013. 113(4): p. 22692308.

30. Venugopalan, V., Pulsed laser ablation of tissue: surface vaporization or thermal explosion?, in Laser-Tissue Interaction VI. 1995, SPIE.

31. Walsh, J.T. and T.F. Deutsch, Pulsed CO2 laser tissue ablation: Measurement of the ablation rate. Lasers in Surgery and Medicine, 1988. 8(3): p. 264-275.

32. Goldbaum, A.M. and J.J. Woog, The $\mathrm{CO} 2$ laser in oculoplastic surgery. Surv Ophthalmol, 1997. 42(3): p. 255-67.

33. Balog, J., et al., Intraoperative Tissue Identification Using Rapid Evaporative Ionization Mass Spectrometry. Science Translational Medicine, 2013. 5(194): p. 194ra93-194ra93.

34. Balog, J., et al., Identification of Biological Tissues by Rapid Evaporative Ionization Mass Spectrometry. Analytical Chemistry, 2010. 82(17): p. 73437350.

35. Niessen, W.M.A., MS-MS and MSn A2 - Lindon, John C, in Encyclopedia of Spectroscopy and Spectrometry (Second Edition). 1999, Academic Press: Oxford. p. 1675-1681.

36. Kyle, P.B., Chapter 7 - Toxicology: GCMS A2 - Nair, Hari, in Mass Spectrometry for the Clinical Laboratory, W. Clarke, Editor. 2017, Academic Press: San Diego. p. 131-163.

37. Wiley, W.C. and I.H. McLaren, Time-of-flight mass spectrometer with improved resolution (Reprinted from Review of Scientific Instruments vol 26, pg 1150, 1995). Journal of Mass Spectrometry, 1997. 32(1): p. 4-11.

38. El-Aneed, A., A. Cohen, and J. Banoub, Mass Spectrometry, Review of the Basics: Electrospray, MALDI, and Commonly Used Mass Analyzers. Applied Spectroscopy Reviews, 2009. 44(3): p. 210-230.

39. https://www.thermofisher.com/

40. Scigelova, M. and A. Makarov, Orbitrap mass analyzer - Overview and applications in proteomics. Proteomics, 2006: p. 16-21.

41. Marshall, A.G., Fourier transform ion cyclotron resonance mass spectrometry - Preface. International Journal of Mass Spectrometry and Ion Processes, 1996. 157: p. R9-R11.

42. Marshall, A.G., Fourier-Transform Ion-Cyclotron Resonance MassSpectrometry Retrospect and Prospects. Abstracts of Papers of the American Chemical Society, 1995. 209: p. 78-Anyl.

43. Marshall, A.G., et al., Fourier transform ion cyclotron resonance mass spectrometry: State of the art. Abstracts of Papers of the American Chemical Society, 2014. 247.

44. Marshall, A.G., C.L. Hendrickson, and M.R. Emmett, Fourier transform ion cyclotron resonance mass spectrometry: $A$ celebration of the power of electrospray ionization. Abstracts of Papers of the American Chemical Society, 2000. 220: p. U98-U98. 
45. Micioni Di Bonaventura, M.V., et al., Evaluation of the hypocholesterolemic effect and prebiotic activity of a lentil (Lens culinaris Medik) extract. Mol Nutr Food Res, 2017. 61(11).

46. Holt, R.I., I. Erotokritou-Mulligan, and P.H. Sonksen, The history of doping and growth hormone abuse in sport. Growth Horm IGF Res, 2009. 19(4): $p$. 320-6.

47. Wong, J.K.Y. and T.S.M. Wan, Doping control analyses in horseracing: $A$ clinician's guide. The Veterinary Journal, 2014. 200(1): p. 8-16.

48. Caron, P., V. Turcotte, and C. Guillemette, A chromatography/tandem mass spectrometry method for the simultaneous profiling of ten endogenous steroids, including progesterone, adrenal precursors, androgens and estrogens, using low serum volume. Steroids, 2015. 104: p. 16-24.

49. Genangeli, M., et al., Development and application of a UHPLC-MS/MS method for the simultaneous determination of 17 steroidal hormones in equine serum. J Mass Spectrom, 2017. 52(1): p. 22-29.

50. GÖRÖG, S., Recent Advances in the Analysis of Steroid Hormones and Related Drugs. Analytical Sciences, 2004. 20(5): p. 767-782.

51. Heding, L.G., Specific and Direct Radioimmunoassay for Human Proinsulin in Serum, in Radioimmunoassays for Insulin, C-Peptide and Proinsulin. 1988, Springer Netherlands. p. 56-63.

52. Stanczyk, F.Z., J. Jurow, and A.W. Hsing, Limitations of Direct Immunoassays for Measuring Circulating Estradiol Levels in Postmenopausal Women and Men in Epidemiologic Studies. Cancer Epidemiology Biomarkers \& Prevention, 2010. 19(4): p. 903-906.

53. Taieb, J., Testosterone Measured by 10 Immunoassays and by IsotopeDilution Gas Chromatography-Mass Spectrometry in Sera from 116 Men, Women, and Children. Clinical Chemistry, 2003. 49(8): p. 1381-1395.

54. Thomas-Jones, E., et al., DYNAMICS OF ESTROGEN BIOMARKER RESPONSES IN RAINBOW TROUT EXPOSED TO 17ß-ESTRADIOL AND 17aETHINYLESTRADIOL. Environmental Toxicology and Chemistry, 2003. 22(12): p. 3001.

55. Choi, T.L.S., et al., Detection of seventy-two anabolic and androgenic steroids and/or their esters in horse hair using ultra-high performance liquid chromatography-high resolution mass spectrometry in multiplexed targeted MS 2 mode and gas chromatography-tandem mass spectrometry. Journal of Chromatography A, 2018. 1566: p. 51-63.

56. Wong, J.K.Y., et al., Doping control analysis of 121 prohibited substances in equine hair by liquid chromatography-tandem mass spectrometry. Journal of Pharmaceutical and Biomedical Analysis, 2018. 158: p. 189-203.

57. Guan, F., et al., Detection, quantification and confirmation of anabolic steroids in equine plasma by liquid chromatography and tandem mass spectrometry. Journal of Chromatography B, 2005. 829(1-2): p. 56-68.

58. Hintikka, L., Development of Mass Spectrometric Methods for Analysis of Anabolic Androgenic Steroids. 2018, University of Helsinki. 
59. Georgakopoulos, C.G., et al., Preventive doping control analysis: liquid and gas chromatography time-of-flight mass spectrometry for detection of designer steroids. Rapid Communications in Mass Spectrometry, 2007. 21(15): p. 2439-2446.

60. Gray, B., et al., Detection of prohibited substances in equine hair by ultrahigh performance liquid chromatography-triple quadrupole mass spectrometry - application to doping control samples. Drug Testing and Analysis, 2018. 10(7): p. 1050-1060.

61. Ray, J.A., et al., Performance enhancement in the measurement of 5 endogenous steroids by $L C-M S / M S$ combined with differential ion mobility spectrometry. Clinica Chimica Acta, 2015. 438: p. 330-336.

62. Kaabia, Z., et al., Ultra high performance liquid chromatography/tandem mass spectrometry based identification of steroid esters in serum and plasma: An efficient strategy to detect natural steroids abuse in breeding and racing animals. Journal of Chromatography A, 2013. 1284: p. 126-140.

63. Houghton, E., et al., Studies related to the metabolism of anabolic steroids in the horse: a gas chromatographic mass spectrometric method to confirm the administration of 19-nortestosterone or its esters to horses. Biomed Mass Spectrom, 1978. 5(2): p. 170-3.

64. Ke, Y., et al., A sensitive, simple and robust $L C-M S / M S$ method for the simultaneous quantification of seven androgen- and estrogen-related steroids in postmenopausal serum. The Journal of Steroid Biochemistry and Molecular Biology, 2014. 144: p. 523-534.

65. Yamashita, K., et al., Development of sensitive derivatization method for aldosterone in liquid chromatography-electrospray ionization tandem mass spectrometry of corticosteroids. Journal of Chromatography A, 2008. 1200(2): p. 114-121.

66. Magnisali, P., et al., Routine method for the simultaneous quantification of 17a-hydroxyprogesterone, testosterone, dehydroepiandrosterone, androstenedione, cortisol, and pregnenolone in human serum of neonates using gas chromatography-mass spectrometry. Journal of Chromatography A, 2008. 1206(2): p. 166-177.

67. Büttler, R.M., et al., Simultaneous measurement of testosterone, androstenedione and dehydroepiandrosterone (DHEA) in serum and plasma using Isotope-Dilution 2-Dimension Ultra High Performance Liquid-Chromatography Tandem Mass Spectrometry (ID-LC-MS/MS). Clinica Chimica Acta, 2015. 438: p. 157-159.

68. Peitzsch, M., et al., An LC-MS/MS method for steroid profiling during adrenal venous sampling for investigation of primary aldosteronism. The Journal of Steroid Biochemistry and Molecular Biology, 2015. 145: p. 7584.

69. He, G., Y. Wu, and J. Lu, Doping control analysis of 13 steroids and structural-like analytes in human urine using Quadrupole-Orbitrap LC- 
MS/MS with paralle/ reaction monitoring (PRM) mode. Steroids, 2018. 131: p. 1-6.

70. Karatt, T.K., et al., Mass spectrometric method for distinguishing isomers of dexamethasone via fragment mass ratio: An HRMS approach. Journal of Mass Spectrometry, 2018. 53(11): p. 1046-1058.

71. You, Y., et al., Simultaneous Determination of Testosterone and Testosterone Enanthate in Equine Plasma by UHPLC-MS-MS. Chromatographia, 2010. 72(11-12): p. 1097-1106.

72. Wong, C.H., et al., Rapid screening of anabolic steroids in horse urine with ultra-high-performance liquid chromatography/tandem mass spectrometry after chemical derivatisation. J Chromatogr A, 2012. 1232: p. 257-65.

73. Voelker, S.E., L.M. Lorenz, and J.J. Litzau, Semi-quantitative determination of designer steroids by high-performance liquid chromatography with ultraviolet detection in the absence of reference material. Drug Test Anal, 2018.

74. Wang, Z., et al., A novel HPLC-MRM strategy to discover unknown and longterm metabolites of stanozolol for expanding analytical possibilities in doping-control. Journal of Chromatography B-Analytical Technologies in the Biomedical and Life Sciences, 2017. 1040: p. 250-259.

75. Baglai, A., et al., Enhancing detectability of anabolic-steroid residues in bovine urine by actively modulated online comprehensive twodimensional liquid chromatography - high-resolution mass spectrometry. Analytica Chimica Acta, 2018. 1013: p. 87-97.

76. Pitarch-Motellon, J., et al., Determination of selected endogenous anabolic androgenic steroids and ratios in urine by ultra high performance liquid chromatography tandem mass spectrometry and isotope pattern deconvolution. Journal of Chromatography A, 2017. 1515: p. 172-178.

77. Shao, B., et al., Simultaneous determination of residual hormonal chemicals in meat, kidney, liver tissues and milk by liquid chromatography-tandem mass spectrometry. Analytica Chimica Acta, 2005. 548(1-2): p. 41-50.

78. Ceglarek, U., et al., Rapid quantification of steroid patterns in human serum by on-line solid phase extraction combined with liquid chromatography-triple quadrupole linear ion trap mass spectrometry. Clinica Chimica Acta, 2009. 401(1-2): p. 114-118.

79. IFHA. Available from: http://www.ifhaonline.org/default.asp?section=Racing\&area=0\#a6a.

80. Allis, O., et al., Liquid Chromatography-Tandem Mass Spectrometry Application, for the Determination of Extracellular Hepatotoxins in Irish Lake and Drinking Waters. Analytical Chemistry, 2007. 79(9): p. 34363447.

81. Bonfiglio, R., et al., The effects of sample preparation methods on the variability of the electrospray ionization response for model drug 
compounds. Rapid Communications in Mass Spectrometry, 1999. 13(12): p. $1175-1185$.

82. Andrews, M.A., et al., Physical Effects of Anabolic-androgenic Steroids in Healthy Exercising Adults. Current Sports Medicine Reports, 2018. 17(7): p. 232-241.

83. Armstrong, J.M., et al., Impact of anabolic androgenic steroids on sexual function. Translational Andrology and Urology, 2018. 7(3): p. 483-489.

84. Huang, X., D. Yuan, and B. Huang, Determination of steroid sex hormones in urine matrix by stir bar sorptive extraction based on monolithic material and liquid chromatography with diode array detection. Talanta, 2007.

85. Annesley, T.M., Ion suppression in mass spectrometry. Clin Chem, 2003. 49(7): p. 1041-4.

86. Harrison, A.G., Chemical ionization mass spectrometry. 2nd ed. 1992, Boca Raton, Fla.: CRC Press. 208 p.

87. Zuber, J., et al., Gas Chromatography/Atmospheric Pressure Chemical Ionization-Fourier Transform Ion Cyclotron Resonance Mass Spectrometry of Pyrolysis Oil from German Brown Coal. International Journal of Analytical Chemistry, 2016.

88. Prokai, L. and S.M. Stevens, Direct Analysis in Real Time (DART) of an Organothiophosphate at Ultrahigh Resolution by Fourier Transform Ion Cyclotron Resonance Mass Spectrometry and Tandem Mass Spectrometry. International Journal of Molecular Sciences, 2016. 17(1).

89. ; Available from: http://www.premierbiosoft.com/tech_notes/massspectrometry.html.

90. Wang, Y.N., et al., Metabolic profile of Cortex Fraxini in rats using UHPLC combined with Fourier transform ion cyclotron resonance mass spectrometry. Rsc Advances, 2016. 6(46): p. 39642-39651.

91. Jimenez-Chillaron, J.C., et al., The role of nutrition on epigenetic modifications and their implications on health. Biochimie, 2012. 94(11): p. 2242-63.

92. Kilonzo-Nthenge, S.N.N.a.A.K., Soybean in Monogastric Nutrition: Modifications to Add Value and Disease Prevention Properties. 2013.

93. Ganesan, K. and B. Xu, Polyphenol-Rich Lentils and Their Health Promoting Effects. International Journal of Molecular Sciences, 2017. 18(11): p. 2390.

94. Johnson, C.R., et al., Lentil (Lens culinaris L.): A prebiotic-rich whole food legume. Food Research International, 2013. 51(1): p. 107-113.

95. Lee, S.-O., et al., Soyasaponins Lowered Plasma Cholesterol and Increased Fecal Bile Acids in Female Golden Syrian Hamsters. Experimental Biology and Medicine, 2005. 230(7): p. 472-478.

96. Zhang, W., S.P. Teng, and D.G. Popovich, Generation of group $B$ soyasaponins I and III by hydrolysis. J Agric Food Chem, 2009. 57(9): p. 3620-5. 
97. Zhang, W. and D.G. Popovich, Group B oleanane triterpenoid extract containing soyasaponins / and III from soy flour induces apoptosis in HepG2 cells. J Agric Food Chem, 2010. 58(9): p. 5315-9.

98. Gurfinkel, D.M. and A.V. Rao, Determination of Saponins in Legumes by Direct Densitometry. Journal of Agricultural and Food Chemistry, 2002. 50(3): p. 426-430.

99. Li, D., et al., Microbial Biogeography and Core Microbiota of the Rat Digestive Tract. Scientific Reports, 2017. 7(1).

100. Martínez-Augustin, O. and F.S.d. Medina, Intestinal bile acid physiology and pathophysiology. World Journal of Gastroenterology, 2008. 14(37): p. 5630.

101. Chiang, J.Y.L., Bile Acid Metabolism and Signaling, in Comprehensive Physiology. 2013, John Wiley \& Sons, Inc.

102. Suzuki, Y., et al., Simple and rapid quantitation of 21 bile acids in rat serum and liver by UPLC-MS-MS: effect of high fat diet on glycine conjugates of rat bile acids. Nagoya J Med Sci, 2013. 75(1-2): p. 57-71.

103. Singhal, N., et al., MALDI-TOF mass spectrometry: an emerging technology for microbial identification and diagnosis. Frontiers in Microbiology, 2015.

6.

104. Patel, R., MALDI-TOF MS for the Diagnosis of Infectious Diseases. Clinical Chemistry, 2014. 61(1): p. 100-111.

105. Ščupáková, K., et al., Spatial Systems Lipidomics Reveals Nonalcoholic Fatty Liver Disease Heterogeneity. Analytical Chemistry, 2018. 90(8): p. 5130-5138.

106. Scupakova, K., et al., Spatial Systems Lipidomics Reveals Nonalcoholic Fatty Liver Disease Heterogeneity. Anal Chem, 2018. 90(8): p. 5130-5138.

107. Flinders, B., et al., Cross-Species Molecular Imaging of Bile Salts and Lipids in Liver: Identification of Molecular Structural Markers in Health and Disease. Anal Chem, 2018. 90(20): p. 11835-11846.

108. Gessel, M.M., J.L. Norris, and R.M. Caprioli, MALDI imaging mass spectrometry: spatial molecular analysis to enable a new age of discovery. J Proteomics, 2014. 107: p. 71-82.

109. Zavalin, A., et al., Direct imaging of single cells and tissue at sub-cellular spatial resolution using transmission geometry MALDIMS. Journal of Mass Spectrometry, 2012. 47(11): p. 1473-1481.

110. Gustafsson, J.O.R., et al., MALDI Imaging Mass Spectrometry (MALDIIMS)—Application of Spatial Proteomics for Ovarian Cancer Classification and Diagnosis. International Journal of Molecular Sciences, 2011. 12(1): $\mathrm{p}$. 773-794.

111. Vaysse, P.M., et al., Mass spectrometry imaging for clinical research-latest developments, applications, and current limitations. Analyst, 2017. 142(15): p. 2690-2712.

112. Lennart R.S. Huizing, S.R.E., Bart W.A.M.M Beulen, Florian P.Y Barré, Paul B. Kwant, Rob J. Vreeken, Ron M.A. Heeren, Matching sample preparation 
speed to high throughput mass spectrometry imaging. Submitted to Clinical Mass Spectrometry (2018), 2018.

113. Sladkova, K., J. Houska, and J. Havel, Laser desorption ionization of red phosphorus clusters and their use for mass calibration in time-of-flight mass spectrometry. Rapid Communications in Mass Spectrometry, 2009. 23(19): p. 3114-3118.

114. Ettcheto, M., et al., Hypercholesterolemia and neurodegeneration. Comparison of hippocampal phenotypes in LDLr knockout and APPSwe/PS1dE9 mice. Experimental Gerontology, 2015. 65: p. 69-78.

115. Ullrich, C., M. Pirchl, and C. Humpel, Hypercholesterolemia in rats impairs the cholinergic system and leads to memory deficits. Molecular and Cellular Neuroscience, 2010. 45(4): p. 408-417.

116. Sandhoff, R., et al., Determination of cholesterol at the low picomole level by nano-electrospray ionization tandem mass spectrometry. J Lipid Res, 1999. 40(1): p. 126-32.

117. Sagratini, G., et al., Quantification of Soyasaponins / and $\beta \mathrm{g}$ in Italian Lentil Seeds by Solid-Phase Extraction (SPE) and High-Performance Liquid Chromatography-Mass Spectrometry (HPLC-MS). Journal of Agricultural and Food Chemistry, 2009. 57(23): p. 11226-11233.

118. Waluk, D., Biosynthesis and physiological functions of $\mathrm{N}$-acyl amino acids. Microbiology and Toxicology, 2012.

119. Connor, M., C.W. Vaughan, and R.J. Vandenberg, N-Acyl amino acids and $N$-acyl neurotransmitter conjugates: neuromodulators and probes for new drug targets. British Journal of Pharmacology, 2010. 160(8): p. 18571871.

120. Gérard, P., Metabolism of Cholesterol and Bile Acids by the Gut Microbiota. Pathogens, 2013. 3(1): p. 14-24.

121. Murakami, S., et al., Taurine ameliorates cholesterol metabolism by stimulating bile acid production in high-cholesterol-fed rats. Clinical and Experimental Pharmacology and Physiology, 2016. 43(3): p. 372-378.

122. Merheb, M., et al., Taurine Intestinal Absorption and Renal Excretion Test in Diabetic Patients: A pilot study. Diabetes Care, 2007. 30(10): p. 2652-2654.

123. Murakami, S., et al., Effect of taurine on cholesterol metabolism in hamsters: Up-regulation of low density lipoprotein $(L D L)$ receptor by taurine. Life Sciences, 2002. 70(20): p. 2355-2366.

124. Kálmán, J., et al., High cholesterol diet down regulates the activity of activator protein-1 but not nuclear factor-kappa $B$ in rabbit brain. Life Sciences, 2001. 68(13): p. 1495-1503.

125. Nestel, P.J., P.H. Schreibman, and E.H. Ahrens, Cholesterol Metabolism in Human Obesity. Journal of Clinical Investigation, 1973. 52(10): p. 23892397.

126. Zhang, J. and Q. Liu, Cholesterol metabolism and homeostasis in the brain. Protein \& Cell, 2015. 6(4): p. 254-264. 
127. Shariatgorji, M., P. Svenningsson, and P.E. Andrén, Mass spectrometry imaging, an emerging technology in neuropsychopharmacology. Neuropsychopharmacology : official publication of the American College of Neuropsychopharmacology, 2014. 39(1): p. 34-49.

128. Kriegsmann, J., Kriegsmann, M., \& Casadonte, R., MALDI TOF imaging mass spectrometry in clinical pathology: A valuable tool for cancer diagnostics (Review). International Journal of Oncology, 2014. 46(3): p. 893-906.

129. Seeley, E.H. and R.M. Caprioli, Imaging mass spectrometry: Towards clinical diagnostics. Proteomics Clin Appl, 2008. 2(10-11): p. 1435-43.

130. Mascini, N.E., et al., The Use of Mass Spectrometry Imaging to Predict Treatment Response of Patient-Derived Xenograft Models of TripleNegative Breast Cancer. Journal of Proteome Research, 2015. 14(2): p. 1069-1075.

131. Cornett, D.S., et al., A Novel Histology-directed Strategy for MALDI-MS Tissue Profiling That Improves Throughput and Cellular Specificity in Human Breast Cancer. Molecular \&amp; Cellular Proteomics, 2006. 5(10): p. 1975-1983.

132. Organization, W.H. Cancer. 2019; Available from: https://www.who.int/news-room/fact-sheets/detail/cancer.

133. Chen, A.M., et al., Breast conservation after neoadjuvant chemotherapy. Cancer, 2005. 103(4): p. 689-95.

134. Buchholz, T.A., et al., Neoadjuvant chemotherapy for breast carcinoma: multidisciplinary considerations of benefits and risks. Cancer, 2003. 98(6): p. 1150-60.

135. Oncoline. Mammacarcinoom: landelijke richtlijn risicoprofilering. 2012 [cited 2017; Available from: http://www.oncoline.nl/mammacarcinoom.

136. Straver, M.E., et al., The Relevance of Breast Cancer Subtypes in the Outcome of Neoadjuvant Chemotherapy. Annals of Surgical Oncology, 2010. 17(9): p. 2411-2418.

137. Kaletas, B.K., et al., Sample preparation issues for tissue imaging by imaging MS. Proteomics, 2009. 9(10): p. 2622-33.

138. Goodwin, R.J., Sample preparation for mass spectrometry imaging: small mistakes can lead to big consequences. J Proteomics, 2012. 75(16): p. 4893-911.

139. Vos, D.R.N., et al., Strategies for managing multi-patient 3D mass spectrometry imaging data. Journal of Proteomics, 2019. 193: p. 184-191.

140. Ly, A., et al., Site-to-Site Reproducibility and Spatial Resolution in MALDIMSI of Peptides from Formalin-Fixed Paraffin-Embedded Samples. PROTEOMICS - Clinical Applications, 2019. 13(1): p. 1800029.

141. Buck, A., et al., Round robin study of formalin-fixed paraffin-embedded tissues in mass spectrometry imaging. Analytical and bioanalytical chemistry, 2018. 410(23): p. 5969-5980.

142. Pedrini, J.L., MD, PhD, How can we predict pathological complete response in breast cancer patients after neoadjuvant chemotherapy. 2014, Research Gate. 
143. Strohalm, M., et al., mMass data miner: an open source alternative for mass spectrometric data analysis. Rapid Communications in Mass Spectrometry, 2008. 22(6): p. 905-908.

144. Chong, J.a.X., J, MetaboAnalystR: an $R$ package for flexible and reproducible analysis of metabolomics data. Bioinformatics, 2018. 27: $p$. 4313-4314.

145. van den Berg, R.A., et al., Centering, scaling, and transformations: improving the biological information content of metabolomics data. BMC Genomics, 2006. 7: p. 142.

146. Deininger, S.O., et al., Normalization in MALDI-TOF imaging datasets of proteins: practical considerations. Analytical and Bioanalytical Chemistry, 2011. 401(1): p. 167-181.

147. Diehl, H.C., et al., The challenge of on-tissue digestion for MALDI MSI- a comparison of different protocols to improve imaging experiments. Anal Bioanal Chem, 2015. 407(8): p. 2223-43.

148. Heijs, B., et al., Brain Region-Specific Dynamics of On-Tissue Protein Digestion Using MALDIMass Spectrometry Imaging. J Proteome Res, 2015. 14(12): p. 5348-54.

149. Casadonte, R. and R.M. Caprioli, Proteomic analysis of formalin-fixed paraffin-embedded tissue by MALDI imaging mass spectrometry. Nat Protoc, 2011. 6(11): p. 1695-709.

150. Groseclose, M.R., et al., High-throughput proteomic analysis of formalinfixed paraffin-embedded tissue microarrays using MALDI imaging mass spectrometry. Proteomics, 2008. 8(18): p. 3715-24.

151. Erich, K., et al., Scores for standardization of on-tissue digestion of formalin-fixed paraffin-embedded tissue in MALDI-MS imaging. Biochimica et Biophysica Acta (BBA) - Proteins and Proteomics, 2017. 1865(7): p. 907-915.

152. Hong, H.X., et al., Quality control and quality assessment of data from surface-enhanced laser desorption/ionization (SELDI) time-of flight (TOF) mass spectrometry (MS). Bmc Bioinformatics, 2005. 6.

153. Kumar, V., Abbas A, K., \& Aster, J., Robbins Basic Pathology10th Edition ed. 2017.

154. Cortazar, P., et al., Pathological complete response and long-term clinical benefit in breast cancer: the CTNeoBC pooled analysis. The Lancet, 2014. 384(9938): p. 164-172.

155. Ding, L., et al., Clonal evolution in relapsed acute myeloid leukaemia revealed by whole-genome sequencing. Nature, 2012. 481(7382): p. 506510.

156. Ruiz, C., et al., Advancing a clinically relevant perspective of the clonal nature of cancer. Proceedings of the National Academy of Sciences, 2011. 108(29): p. 12054-12059. 
157. Ifa, D.R., et al., Desorption electrospray ionization and other ambient ionization methods: current progress and preview. Analyst, 2010. 135(4): p. 669-81.

158. Perez, C.J., et al., Review and perspectives on the applications of mass spectrometry imaging under ambient conditions. Rapid Commun Mass Spectrom, 2018.

159. Wu, C., et al., Mass spectrometry imaging under ambient conditions. Mass Spectrom Rev, 2013. 32(3): p. 218-43.

160. Ifa, D.R. and L.S. Eberlin, Ambient Ionization Mass Spectrometry for Cancer Diagnosis and Surgical Margin Evaluation. Clin Chem, 2016. 62(1): p. 11123.

161. Takats, Z., Mass Spectrometry Sampling Under Ambient Conditions with Desorption Electrospray Ionization. Science, 2004. 306(5695): p. 471-473.

162. Nemes, P. and A. Vertes, Atmospheric-pressure molecular imaging of biological tissues and biofilms by LAESI mass spectrometry. J Vis Exp, 2010(43).

163. Zhang, J.L., et al., Nondestructive tissue analysis for ex vivo and in vivo cancer diagnosis using a handheld mass spectrometry system. Science Translational Medicine, 2017. 9(406).

164. Fatou, B., et al., Real time and in vivo pharmaceutical and environmental studies with SpiderMass instrument. Journal of Biotechnology, 2018. 281 : p. 61-66.

165. Fatou, B., et al., In vivo Real-Time Mass Spectrometry for Guided Surgery Application. Sci Rep, 2016. 6: p. 25919.

166. Saudemont, P., et al., Real-Time Molecular Diagnosis of Tumors Using Water-Assisted Laser Desorption/lonization Mass Spectrometry Technology. Cancer Cell, 2018. 34(5): p. 840-+.

167. Woolman, M., et al., Rapid determination of medulloblastoma subgroup affiliation with mass spectrometry using a handheld picosecond infrared laser desorption probe. Chem Sci, 2017. 8(9): p. 6508-6519.

168. Woolman, M., et al., Optimized Mass Spectrometry Analysis Workflow with Polarimetric Guidance for ex vivo and in situ Sampling of Biological Tissues. Sci Rep, 2017. 7(1): p. 468.

169. Woolman, M., et al., Optimized Mass Spectrometry Analysis Workflow with Polarimetric Guidance for ex vivo and in situ Sampling of Biological Tissues. Scientific Reports, 2017. 7.

170. Balog, J., et al., Identification of the Species of Origin for Meat Products by Rapid Evaporative Ionization Mass Spectrometry. Journal of Agricultural and Food Chemistry, 2016. 64(23): p. 4793-4800.

171. Kailasa, S.K., et al., Progress of electrospray ionization and rapid evaporative ionization mass spectrometric techniques for the broadrange identification of microorganisms. Analyst, 2019. 144(4): p. 10731103. 
172. Golf, O., et al., Rapid Evaporative Ionization Mass Spectrometry Imaging Platform for Direct Mapping from Bulk Tissue and Bacterial Growth Media. Analytical Chemistry, 2015. 87(5): p. 2527-2534.

173. Schäfer, K.-C., et al., In Vivo, In Situ Tissue Analysis Using Rapid Evaporative Ionization Mass Spectrometry. Angewandte Chemie International Edition, 2009. 48(44): p. 8240-8242.

174. St John, E.R., et al., Intraoperative tissue identification by mass spectrometric technologies. TrAC Trends in Analytical Chemistry, 2016. 85: p. 2-9.

175. Cordero Hernandez, Y., et al., Targeted Feature Extraction in MALDI Mass Spectrometry Imaging to Discriminate Proteomic Profiles of Breast and Ovarian Cancer. Proteomics Clin Appl, 2019. 13(1): p. e1700168.

176. Lanzafame, R.J., et al., Comparison of Continuous-Wave, Chop-Wave, and Super Pulse Laser Wounds. Lasers in Surgery and Medicine, 1988. 8(2): $\mathrm{p}$. 119-124.

177. Huizing, L.R.S., et al., Development and evaluation of matrix application techniques for high throughput mass spectrometry imaging of tissues in the clinic. Clinical Mass Spectrometry, 2019. 12: p. 7-15.

178. Bodai, Z., et al., Effect of Electrode Geometry on the Classification Performance of Rapid Evaporative Ionization Mass Spectrometric (REIMS) Bacterial Identification. J Am Soc Mass Spectrom, 2018. 29(1): p. 26-33.

179. Niu, S.F., W.Z. Zhang, and B.T. Chait, Direct comparison of infrared and ultraviolet wavelength matrix-assisted laser desorption/ionization mass spectrometry of proteins. Journal of the American Society for Mass Spectrometry, 1998. 9(1): p. 1-7.

180. Woods, A.S., et al., IR-MALDI-LDI combined with ion mobility orthogonal time-of-flight mass spectrometry. J Proteome Res, 2006. 5(6): p. 1484-7.

181. Rohlfing, A., et al., IR-MALDI-MS analysis of HPTLC-separated phospholipid mixtures directly from the TLC plate. Anal Chem, 2007. 79(15): p. 5793808.

182. Pirro, V., et al., Lipid characterization of individual porcine oocytes by dual mode DESI-MS and data fusion. Analytica Chimica Acta, 2014. 848: p. 5160.

183. Uzbekova, S., et al., MALDI Mass Spectrometry Imaging of Lipids and Gene Expression Reveals Differences in Fatty Acid Metabolism between Follicular Compartments in Porcine Ovaries. Biology (Basel), 2015. 4(1): p. 216-36.

184. Eibisch, M., et al., Phosphatidylcholine dimers can be easily misinterpreted as cardiolipins in complex lipid mixtures: a matrix-assisted laser desorption/ionization time-of-flight mass spectrometric study of lipids from hepatocytes. Rapid Communications in Mass Spectrometry, 2011. 25(18): p. 2619-2626.

185. Bishop, C.M., Pattern recognition and machine learning. 2006: springer. 
186. Dietterich, T.G. and E.B. Kong, Machine learning bias, statistical bias, and statistical variance of decision tree algorithms. 1995, Technical report, Department of Computer Science, Oregon State University.

187. Endo, M. and P.P. Lin, Surgical margins in the management of extremity soft tissue sarcoma. Chin Clin Oncol, 2018. 7(4): p. 37.

188. Greager, J.A., et al., Soft tissue sarcomas of the adult head and neck. Cancer, 1985. 56(4): p. 820-4.

189. Romanach, M.J., et al., Soft tissue sarcomas of the head and neck. SADJ, 2012. 67(10): p. 582-4.

190. Dijkstra, M.D., et al., Soft tissue sarcomas of the head and neck associated with surgical trauma. J Laryngol Otol, 1995. 109(2): p. 126-9.

191. Weber, R.S., et al., Soft tissue sarcomas of the head and neck in adolescents and adults. Am J Surg, 1986. 152(4): p. 386-92.

192. Farhood, A.I., et al., Soft tissue sarcomas of the head and neck in adults. Am J Surg, 1990. 160(4): p. 365-9.

193. Salcedo-Hernandez, R.A., et al., Soft tissue sarcomas of the head and neck. Clinical and pathological evaluation of 108 cases in Mexico. J Craniomaxillofac Surg, 2014. 42(8): p. 1566-71.

194. Kawaguchi, N., et al., The concept of curative margin in surgery for bone and soft tissue sarcoma. Clin Orthop Relat Res, 2004(419): p. 165-72.

195. Kawai, A., et al., Prognostic factors for patients with sarcomas of the pe/vic bones. Cancer, 1998. 82(5): p. 851-9.

196. Buckwalter, J.A. and T.D. Brown, Joint injury, repair, and remodeling: roles in post-traumatic osteoarthritis. Clin Orthop Relat Res, 2004(423): p. 7-16.

197. Anderson, D.D., et al., Post-traumatic osteoarthritis: improved understanding and opportunities for early intervention. J Orthop Res, 2011. 29(6): p. 802-9.

198. Giannini, S., et al., Cartilage repair evolution in post-traumatic osteochondral lesions of the talus: from open field autologous chondrocyte to bone-marrow-derived cells transplantation. Injury, 2010. 41(11): p. 1196-203. 


\subsection{Publications}

This thesis is based on the following publications:

Development and application of a UHPLC-MS/MS method for the simultaneous determination of 17 steroidal hormones in equine serum.

Michele Genangeli, Giovanni Caprioli, Manuela Cortese, Fulvio Laus, Mara Matteucci, Riccardo Petrelli, Massimo Ricciutelli, Gianni Sagratini, Stefano Sartori, Sauro Vittori

Journal of Mass Spectrometry, 2016.52(1): p. 22-29. DOl 10.1002/jms.3896

Simultaneous quantitation of 9 anabolic and natural steroidal hormones in equine urine by UHPLC-MS $\backslash M S$.

Michele Genangeli, Giovanni Caprioli, Manuela Cortese, Fulvio Laus, Riccardo Petrelli, Massimo Ricciutelli, Gianni Sagratini, Stefano Sartori, Sauro Vittori

Journal of Chromatography B, 2019 .1117: p. 36-40. DOI 10.1016/j.jchromb.2019.04.002

Qualification and quantification of 17 endogenous and exogenous steroidal hormones in equine and bovine blood for doping control with UHPLC-MS/MS: a new method.

Michele Genangeli, Giovanni Caprioli, Manuela Cortese, Riccardo Petrelli, Massimo Ricciutelli, Gianni Sagratini, Stefano Sartori, Sauro Vittori

Journal of Chromatography A, 2019 Submitted

MALDI-mass spectrometry imaging to investigate lipid and bile acid modifications caused by lentil extract used as a potential hypocholesterolemic treatment Journal of the American Society for Mass Spectrometry

Michele Genangeli; Annemarie Heijens; Alice Rustichelli; Noortje D. Schuit; Maria Vittoria Micioni Di Bonaventura; Carlo Cifani; Sauro Vittori; Tiffany Porta-Siegel; Ron M. A. Heeren Journal of The American Society for Mass Spectrometry, 2019, DOI: 10.1007/s13361-01902265-9

Tissue classification by Rapid Evaporative lonization Mass Spectrometry (REIMS): comparison between diathermic knife and $\mathrm{CO}_{2}$ laser sampling on classification performances

Michele Genangeli, Ron M.A. Heeren, Tiffany Porta Siegel

Analytical and bioanalytical chemistry, Accepted August 2019

FFPE tissue-based quality control for MALDI-MSI analysis of tryptic peptides and application to predict neoadjuvant therapy response in breast cancer

Caro Thomassen**, Michele Genangeli**, Renée Granzier, Jo Capell, Fanny Boyaval, Loes kooreman, Briete Goorts, Ron M.A. Heeren, Marjolein Smidt, Tiffany Porta Siegel

In preparation

** Shared first authorship

192 
Other Publications:

Detection of endocrine disrupting chemicals and evidence of their effects on the HPG axis of the European anchovy Engraulis encrasicolus.

Andrea Miccoli, Francesca Maradonna, Andrea De Felice, Vincenzo Caputo Barucchia, Andone Estonba, Michele Genangeli, SauroVittori, Iole Leonori, Oliana Carnevalia

Marine environmental research, 2017.127: p137-147. DOI

10.1016/j.marenvres.2017.04.006 


\subsection{Acknowledgments}

Here I am again, thinking about all the amazing people I met in the last few years which made this "adventure" unforgettable.

Acknowledgments are personal, and for this reason, I will add to this book a personalized "thank you". Hope you will appreciate the thought.

Here some 'general' but felt acknowledgments

I must start thanking the person who allowed me to start my Ph.D. in the first place. Dear Prof Vittori, from the bottom of my heart, thank you. From the moment I started my Ph.D., you always supported and helped me to grow personally and professionally. You used to call me "dottó" since the day I became part of your group, and now I can finally see myself with that title. I had an amazing time in Camerino and, since the moment I left I missed all the amazing people in the lab! Thank you, Giovanni, for always supporting and helping me. Thank you Manuela and Max for teaching me everything about MS. Thank you, Gianni, Federica, Pilar and all the other colleagues who always made me feel at home.

And then I flew to the Netherlands... Dear Prof Heeren, it has been a true honor to be part of M4I. Thank you for the big opportunity you gave me and thank you for giving me the possibility of being part of your research group. Thank you, Tiff for being a great supervisor. Your patience and guidance made this possible. I owe you this.

Thanks to all my colleagues at M4i, Surgery, Nanoscopy and IDEE : Alice, Andrew, Anjusha, Anne, Annemarie, Arnoud, Audrey, Bart, Bea, Benjamin, Berta, Brenda, Britt, Bryn, Caitlin, Caro, Christel, Darya, Florian, Frans, Fred, 
Gert, Helen, Jaqueline, Jian-Hua, Jo, Joel, Jonah, Klara, Lennart, Lieke, Livia, Maarten, Maria, Marta, Marty, Maxime, Mirella, Naomi, Nina, Noor, Philippe, Pierre, Pieter, Rob, Ronny, Shane, Stephanie and Sylvia.

Alla mia famiglia. Questa tesi, questo traguardo e' dedicato a voi. Citando Anthony Brandt :" altre cose possono cambiarci, ma iniziamo e finiamo con la famiglia. Gli ultimi anni non sono stati facili; non per me e non per voi. Vi chiedo scusa se non sono ancora tornato, se sono partito e se magari sono stato distante. Siete sempre stati e sarete vicini a me, nel mio cuore qualcunque cosa faccia o abbia fatto.

Mamma e Babbo, siete semplicemente genitori perfetti.

Sofy, da "rompiballe ficcanaso" sei diventata una delle persone piu importanti della mia vita.

Nonna, ho raggiunto questo traguardo anche grazie agli insegnamenti dei 'Nonni'. Sono sicuro che Nonno e' fiero di me.

Anche se non sono piu con me da tempo, sono sicuro che nonna Aura e specialmente nonno Bruno sarebbero fieri di vedere il cognome "Genangeli" seguito da Ph.D. :)

Grazie agli zii: Zia Chicchi, Giugia, Paola, Moreno, Sergio, Laura, Gianni, Rita, Massi e Silvia e grazie ai cugini: Ale, fra, Marco, Luca, Omar

Grazie agli amici di sempre: Andrea, Fiorde, Fiore, Simo, Francio, Nata, Eli's, Ale's, Ele's, Fra's, Giulia's e tutti gli altri che non sto a nominare altrimenti la lista diventerebbe troppo lunga. Riceverete una copia della tesi con dedica personalizzata!

Thanks to all my friends in Maastricht! Without the chill and fun nights I had with you guys, I would not be celebrating today. Yig, Jeroen, Lynnel, Andrew, Jules, Max, GJ, Ollie, Josh, Joep, Isa, Aleix, Lucrezia, Matteo, Albe, Francois, Laura, Marta, Silvia, Nkoli, Fabiana, Valeria, Silvia, Julia, Laura, and many others... Iove you guys. 
Thanks to Marjan, Richard and all the people at Batteraof for making me feel at home and for the wonderful time I had with you guys.

Lizzie, you deserve a chapter. It will be too long to write here about why I want to thank you. You have always been here for me and you literally changed my life. What do you think about me telling you in person why I want to 'thank you' over a Mimosa?:p

Cindy, Sander, Ysable, Ron and Janny. You deserve to be in the acknowledgments as well. Thank you for everything you have done for me in the last few years. You have no idea how much I appreciated it :)

And you, who are reading this chapter right now. If you are not listed above, don't worry. If I gave you this book it means that you are important to me. Thank you! 


\subsection{About the Author}

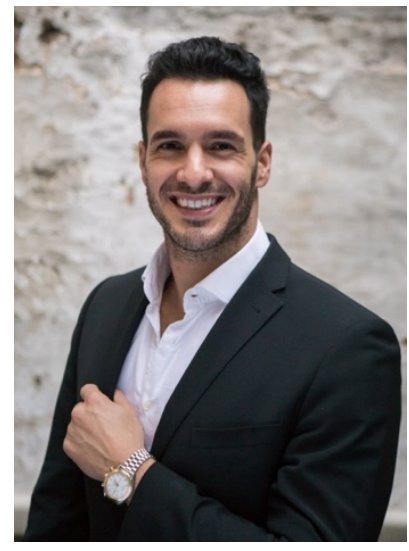

Michele Genangeli was born in Jesi (AN), Italy, on September $29^{\text {th }} 1990$.

He attended the Liceo scientifico tecnologico Leonardo Da Vinci (scientific technological liceum) in Jesi (AN, Italy) from which he obtained his high school diploma in 2009.

From 2009 to 2014 he studied Medicinal Chemistry and Pharmaceutical Technology (C.T.F.) at the University of Camerino - Unicam where he obtained his master's degree (unique course, 5 years).

During his studies, he developed his thesis in a company (Eureka Lab Division, Chiaravalle, Italy) where he developed ready-to-use diagnostic kits for antimycotic drugs in plasma matrix, by HPLC-FD and LC-MS/MS.

Later, under the guidance of Prof. Sauro Vittori, in 2014 he started his Ph.D. at the University of Camerino focusing on the development of ready-to-use diagnostic kits for doping control in biological animal matrices using a UHPLC-MS/MS.

In 2016 he joined the research facility of the Maastricht Multimodal Molecular Imaging Institute - M4I in Maastricht, the Netherlands where, in the early 2017 he started his double degree - Marie Curie fellow Ph.D. program In Multimodal Imaging Mass Spectrometry. There, under the supervision of Prof. Ron M.A. Heeren and Dr. Tiffany Porta, he explored different aspects and application of Mass Spectrometry for diagnostic purposes.

During his Ph.D., he developed a product which in 2018 became an Italian and then European patent (Patent: EP 3026993 A1). 
In 2019 he started a company in the Netherlands (Genax b.v.) based on his invention.

In 2019 he started his postdoctoral position as a business developer at the Leiden Academic Center for Drug Research (LACDR) under the guidance of Prof. dr. Thomas Hankemeier.

The results of the double degree Ph.D. are presented in this thesis. 